Cover: Schematic showing multi-taxa data dictionary.

Overlay photographs: (Left) Red diamond rattlesnake (Crotalus ruber) at San Mateo Creek, San Diego County, California. Photograph taken by Chris W. Brown, U.S. Geological Survey, Western Ecological Resource Center San Diego Field Station, April 24, 1999.

(Top) California red-legged frog (Rana draytonii) at San Francisquito Canyon, Los Angeles County, California. Photograph taken by Chris W. Brown, U.S. Geological Survey, Western Ecological Resource Center San Diego Field Station, July 10, 2002.

(Right) Blainville's horned lizard (Phrynosoma blainvillii) at Torrey Pines State Natural Reserve, San Diego County, California. Photograph taken by Chris W. Brown, U.S. Geological Survey, Western Ecological Resource Center San Diego Field Station, July 26, 1999. 


\section{Multi-Taxa Database Data Dictionary}

By Elise Watson, Carlton J. Rochester, Chris W. Brown, Donn A. Holmes, Stacie A. Hathaway, and Robert N. Fisher

U.S. Geological Survey Western Ecological Research Center

Prepared in cooperation with San Diego Association of Governments (SanDAG)

Techniques and Methods 16-B1 


\section{U.S. Geological Survey, Reston, Virginia: 2021}

For more information on the USGS - the Federal source for science about the Earth, its natural and living resources, natural hazards, and the environment—visit https://www.usgs.gov or call 1-888-ASK-USGS.

For an overview of USGS information products, including maps, imagery, and publications, visit https://store.usgs.gov/.

Any use of trade, firm, or product names is for descriptive purposes only and does not imply endorsement by the U.S. Government.

Although this information product, for the most part, is in the public domain, it also may contain copyrighted materials as noted in the text. Permission to reproduce copyrighted items must be secured from the copyright owner.

Suggested citation:

Watson, E., Rochester, C.J., Brown, C.W., Holmes, D.A., Hathaway, S.A., and Fisher, R.N., 2021, Multi-taxa database data dictionary: U.S. Geological Survey Techniques and Methods 16-B1, 149 p., https://doi.org/10.3133/tm16B1.

ISSN 2328-7055 (online) 


\section{Acknowledgments}

Several people provided input into the design and development of the Multi-Taxa database. We thank Tom Lupo, Ken DeVore, Patrick Gaul, Dave Mayer, Chris Stermer, Colleen Miller, Tony McKinney, Felicia Sirchia, Steve Fancy, Betsy Miller, Keith Greer, Yvonne Moore, Ron Rempel, Sedra Shapiro, Jen Carlino, Stephen Corn, Michael Adams, Jang Byun, Bruce Peterjohn, and Alisa Gallant for their contributions to this project. We would like to thank Lena Lee (National Park Service) and Thomas Burley (U.S. Geological Survey) for their valuable review and feedback on this data dictionary. This is Contribution Number 749 of the U.S. Geological Survey Amphibian Research and Monitoring Initiative.

We thank the following agencies for their support for the Multi-Taxa database: California Department of Fish and Wildlife, U.S. Fish and Wildlife Service, National Park Service, U.S. Geological Survey, City of San Diego, San Diego Association of Governments, San Diego Management and Monitoring Program, and San Diego State University. 



\section{Contents}

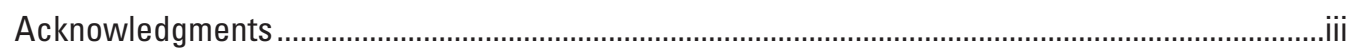

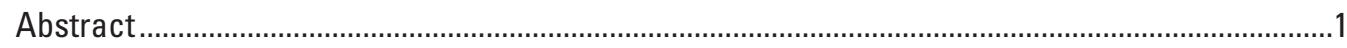

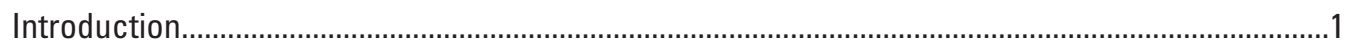

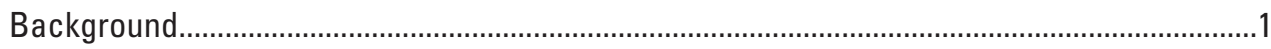

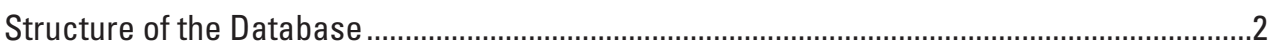

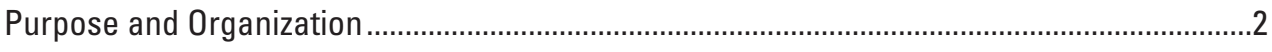

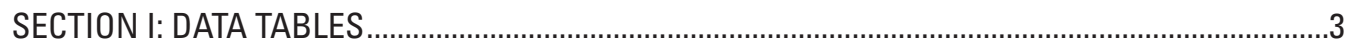

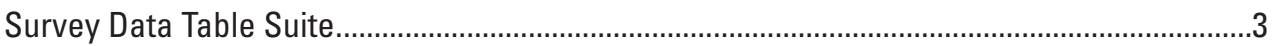

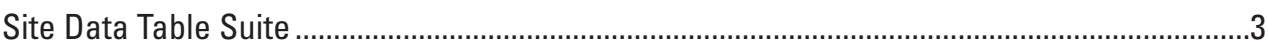

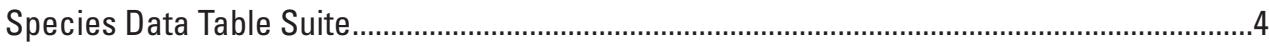

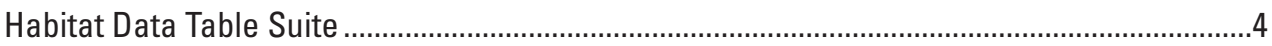

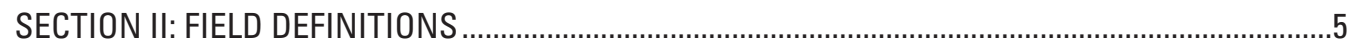

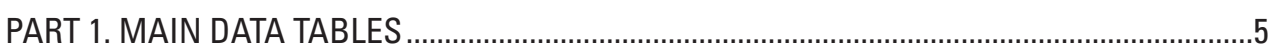

AbundanceEstimates Table..........................................................................................

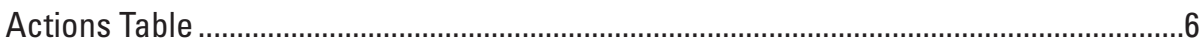

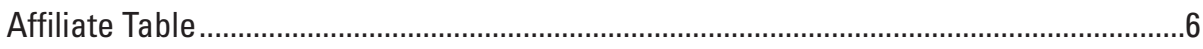

Affiliate_Affiliate Table..................................................................................................

Affiliate_Communication Table...............................................................................

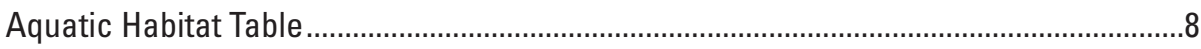

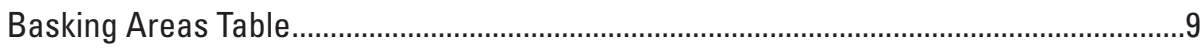

Behaviors Table................................................................................................. 10

Capture Table.................................................................................................... 10

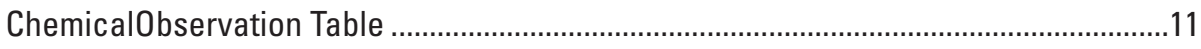

Chemic alTreatment Table ......................................................................................11

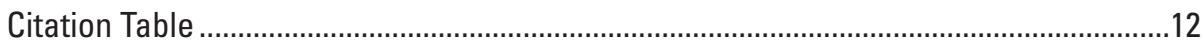

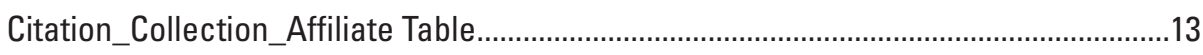

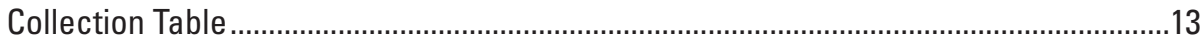

Collection_Affilliate Table.................................................................................................14

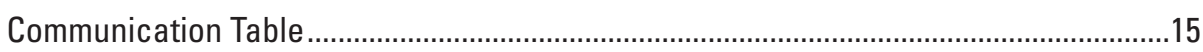

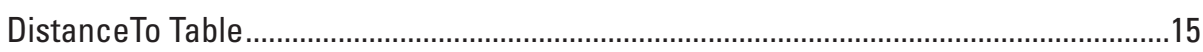

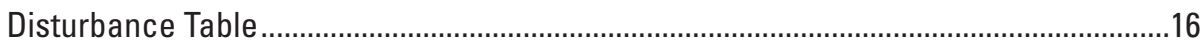

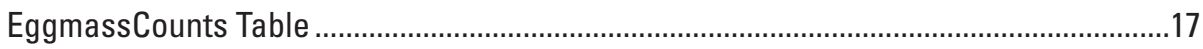

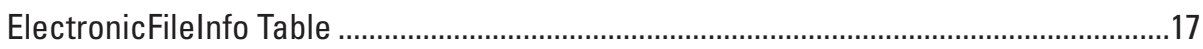

ElectronicFileInfo_Keywords Table ......................................................................18

EstimateSeries Table ............................................................................................

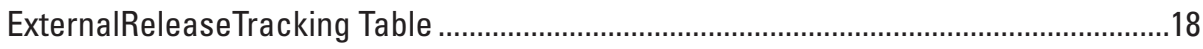

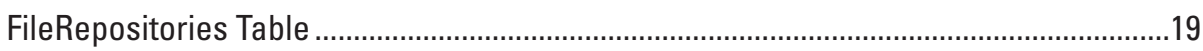

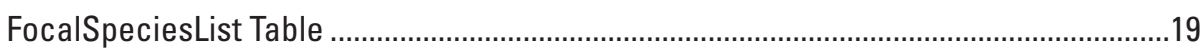

Funding Table

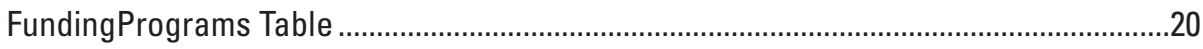

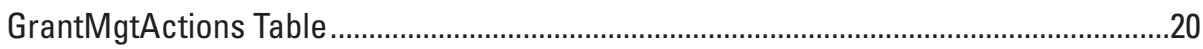

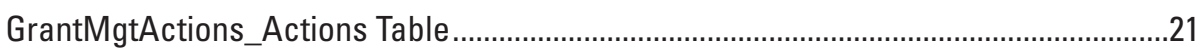

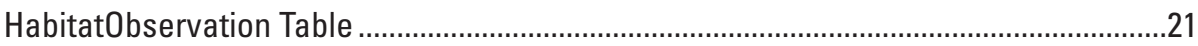




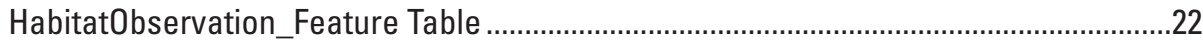

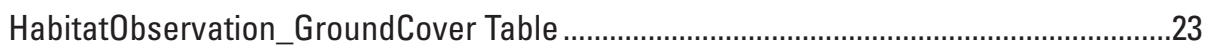

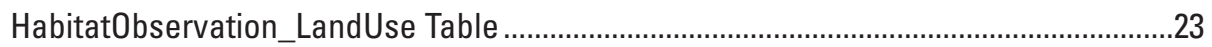

HabitatObservation_SedChemical Table .....................................................................24

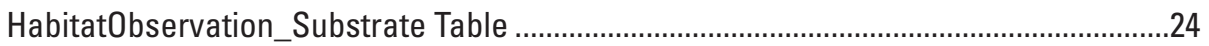

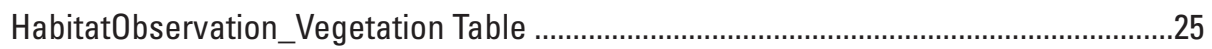

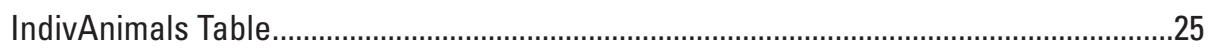

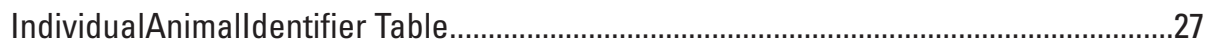

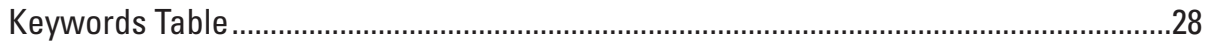

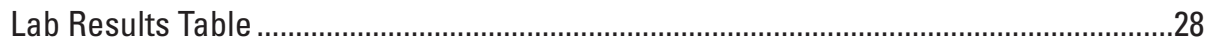

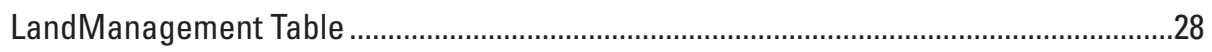

Maintenance Table

Maintenance_Affiliate Table...................................................................................

MaintenancePoints Table.............................................................................................

ManagementActivities Table ................................................................................

ManagementPlans Table ......................................................................................

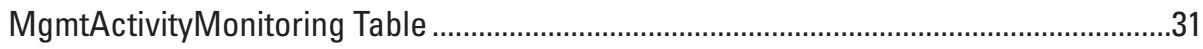

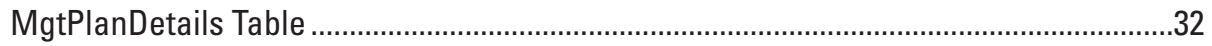

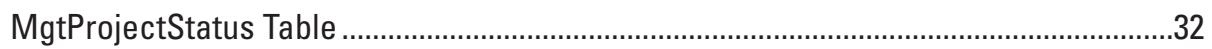

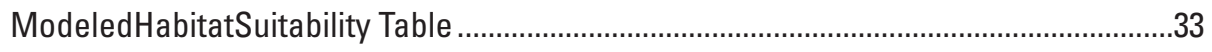

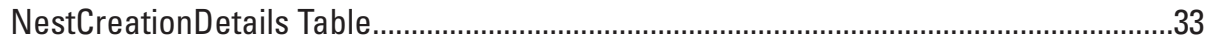

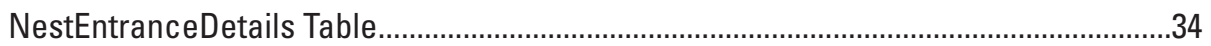

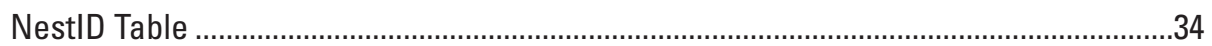

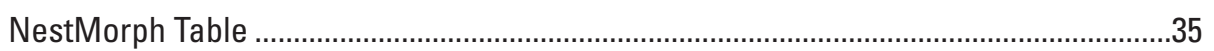

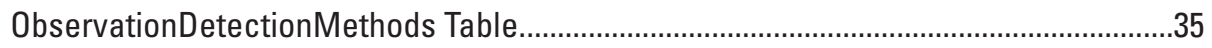

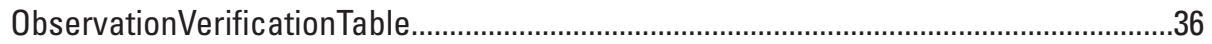

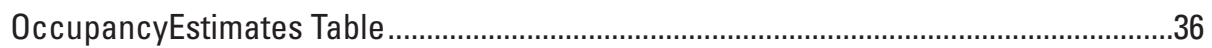

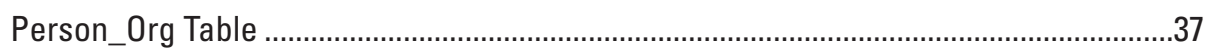

Photolnfo Table …….............................................................................................

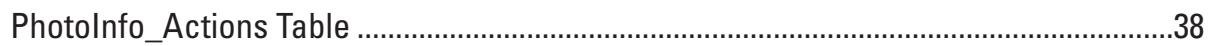

PhotoInfo_HabitatObservations Table ......................................................................

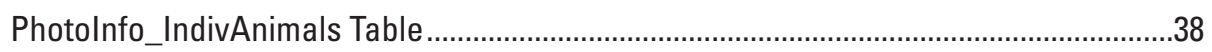

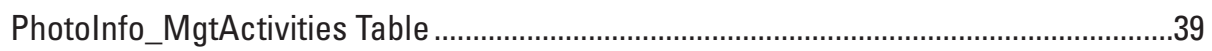

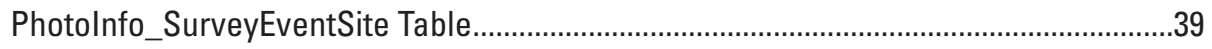

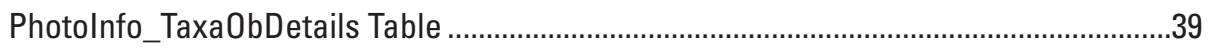

PlantEnhancementDetails Table .............................................................................. 40

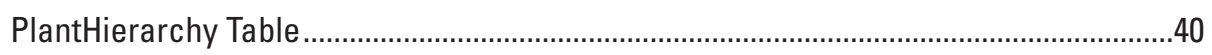

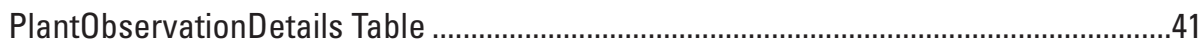

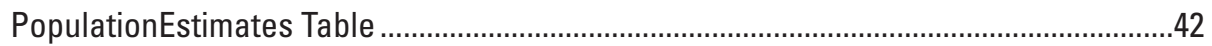

Project_Keywords Table..................................................................................... 42

Project_Project Table.............................................................................................

ProjectID Table

Protocol Table

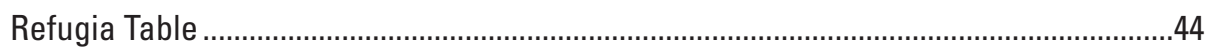

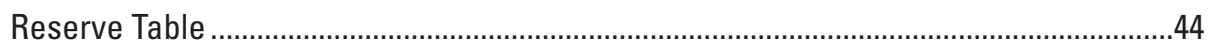




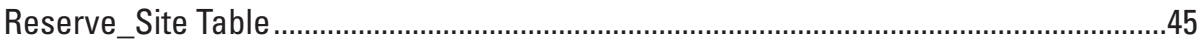

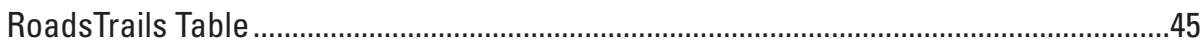

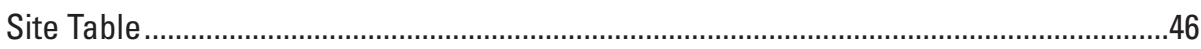

Site_Project Table

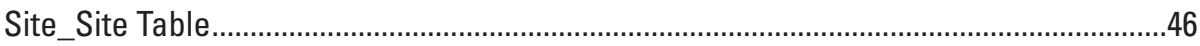

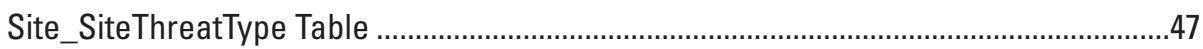

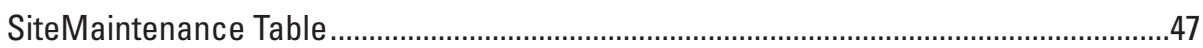

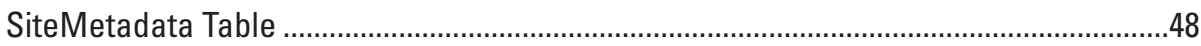

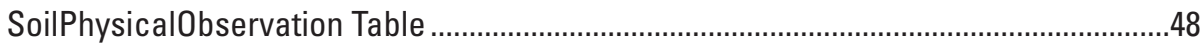

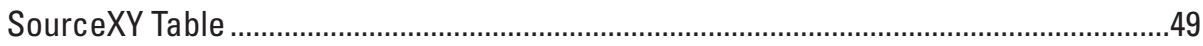

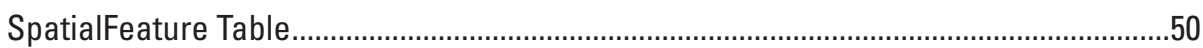

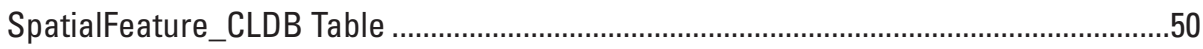

SpeciesHabitatFeature Table................................................................................

Spheroid Table

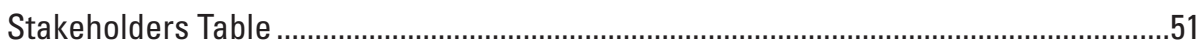

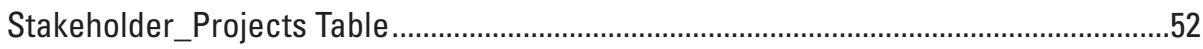

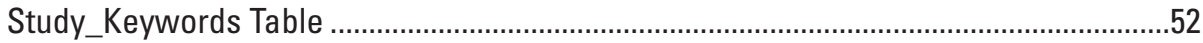

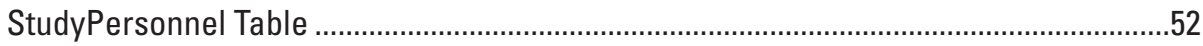

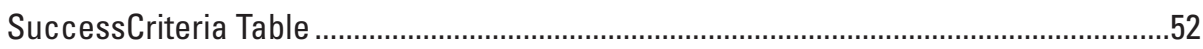

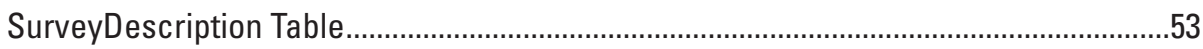

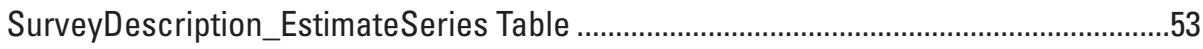

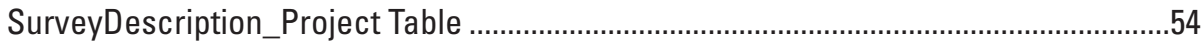

SurveyDescription_Site Table ........................................................................................

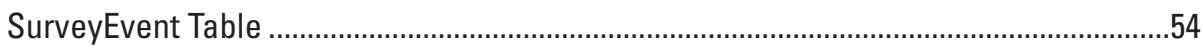

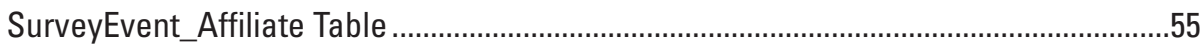

SurveyEvent_Site Table ..........................................................................................

SurveyEvent_SpatialFeature Table ..........................................................................56

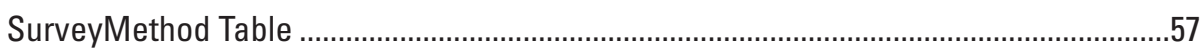

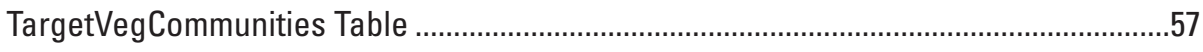

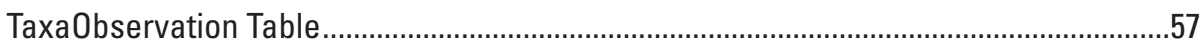

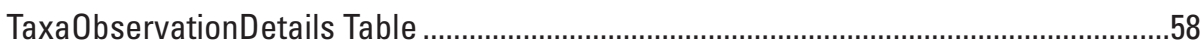

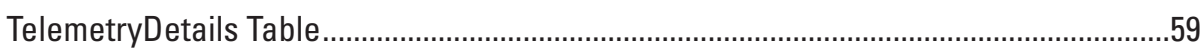

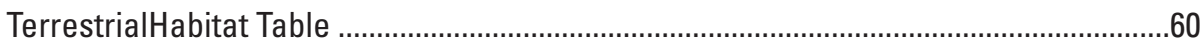

Traps Table

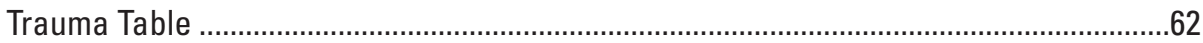

WaterPhysicalObservation Table ................................................................................62

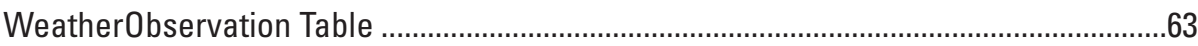

WithinSurveyEffortLost Table ...................................................................................

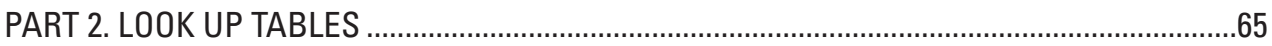

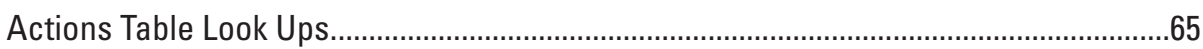

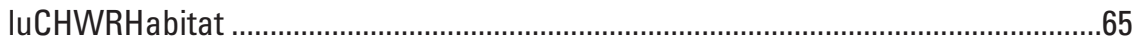

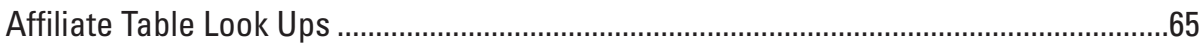

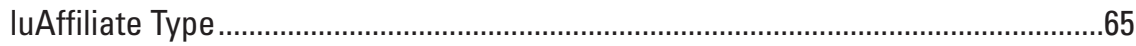

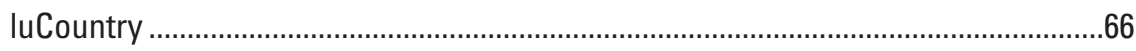

luState 
Aquatic Habitat Table Look Ups …………………....................................................6

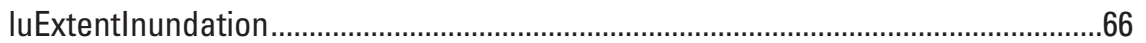

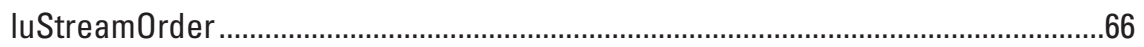

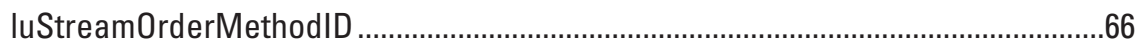

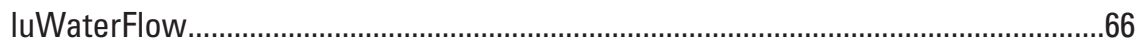

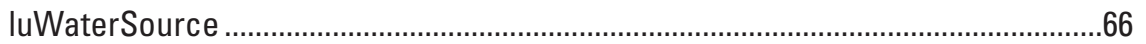

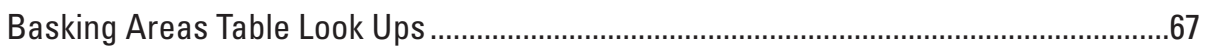

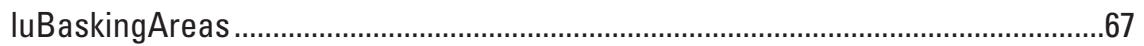

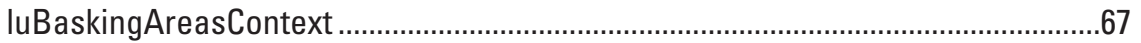

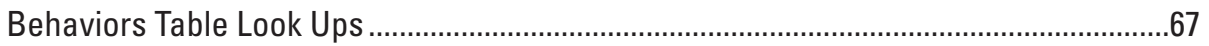

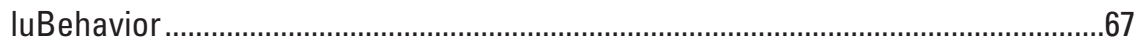

Collection Table Look Ups ...................................................................................

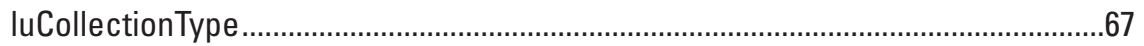

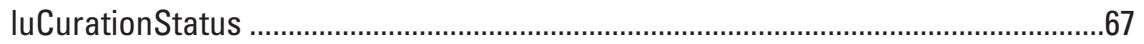

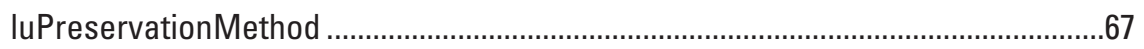

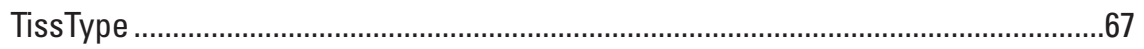

Collection_Affiliate Table Look Ups ..............................................................................68

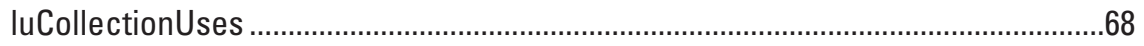

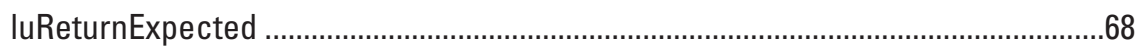

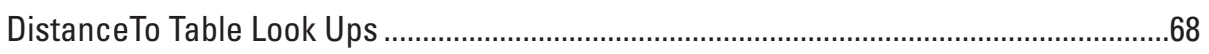

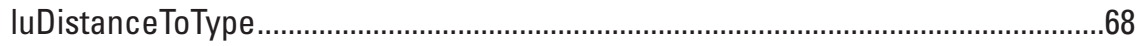

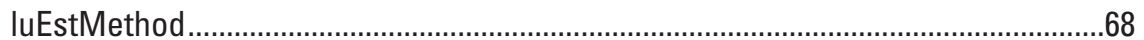

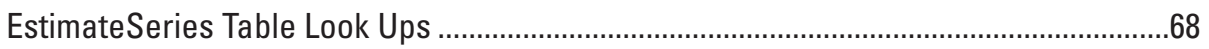

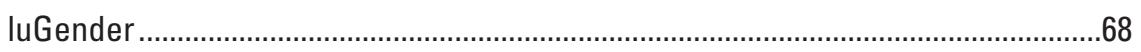

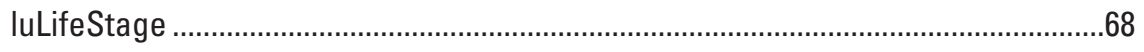

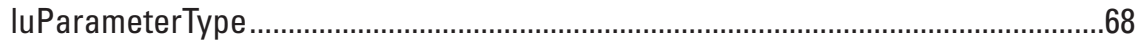

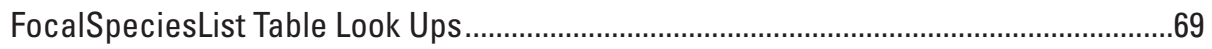

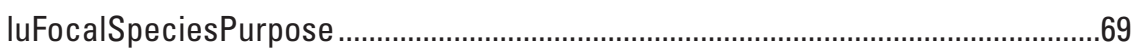

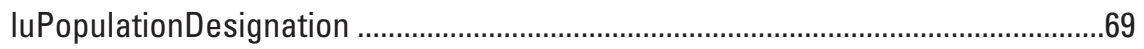

Funding/FundingProgram Table Look Ups ................................................................69

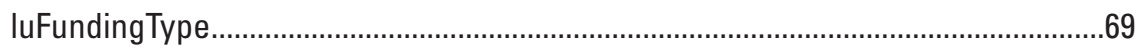

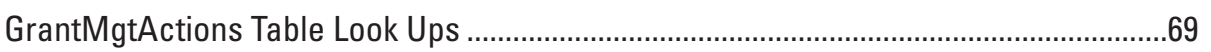

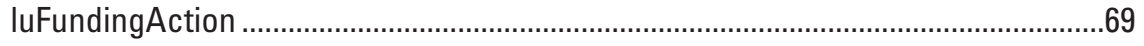

HabitatObservation Table Look Ups...........................................................................69

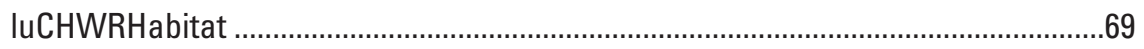

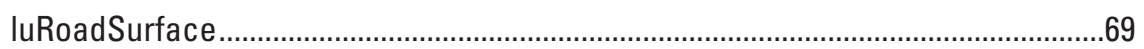

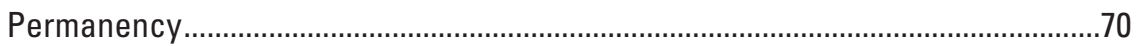

HabitatObservation_Feature Table Look Ups................................................................

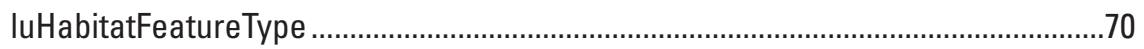

HabitatObservation_GroundCover Table Look Ups....................................................70

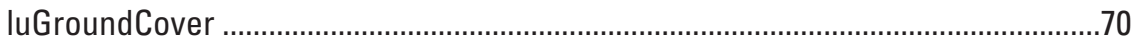

HabitatObservation_LandUse Table Look Ups...............................................................70

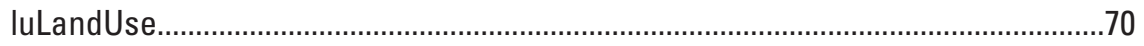

HabitatObservation_SedChemical Table Look Ups.......................................................70

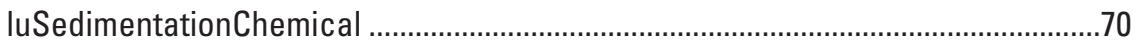


HabitatObservation_Substrate Table Look Ups.............................................................71

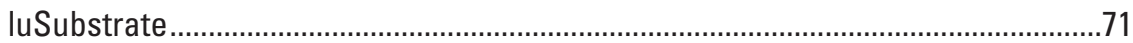

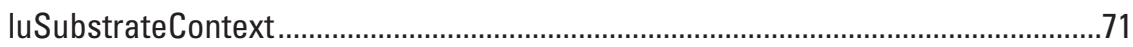

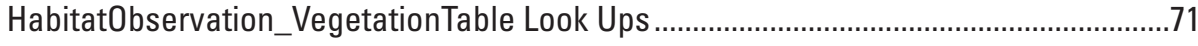

luHabitatObsVegContext....................................................................................

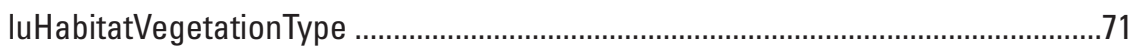

IndivAnimals Table Look Ups ....................................................................................

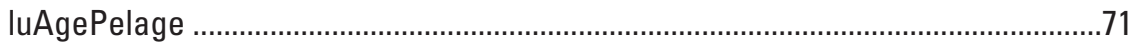

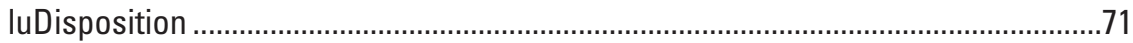

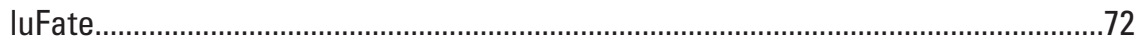

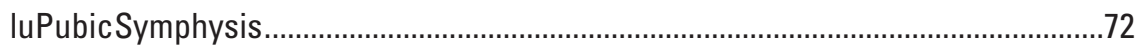

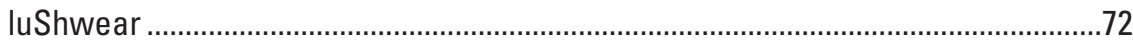

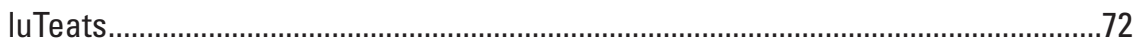

IndividualAnimalldentifier Table Look Ups ...............................................................

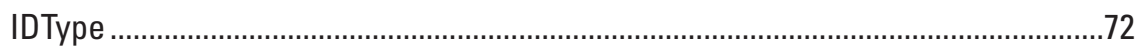

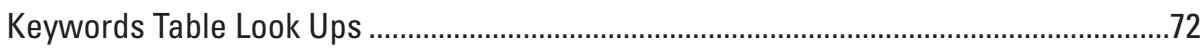

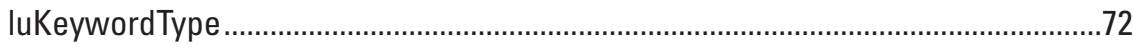

Maintenance Table Look Ups …………………………............................................

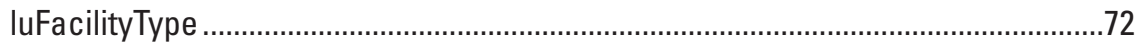

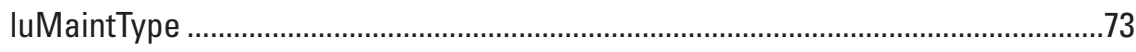

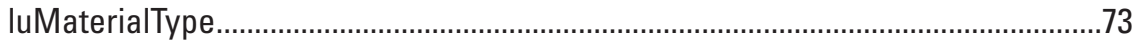

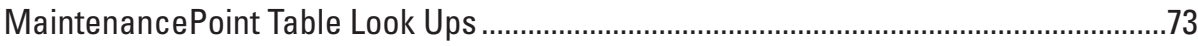

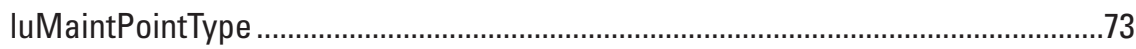

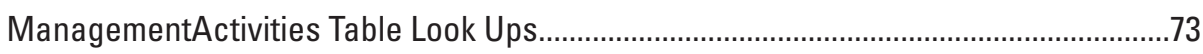

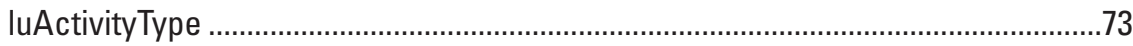

MgmtActivityMonitoring Table Look Ups....................................................................

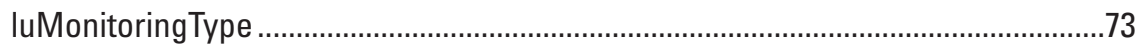

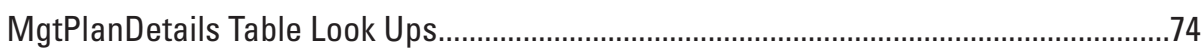

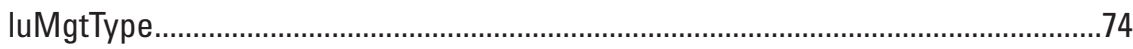

MgtProjectStatus Table Look Ups.............................................................................

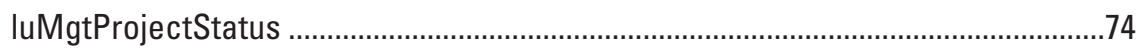

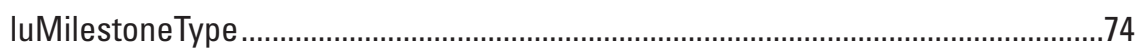

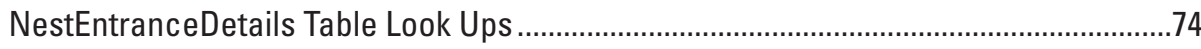

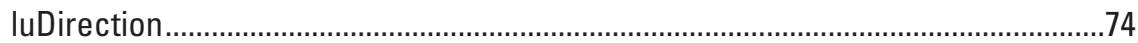

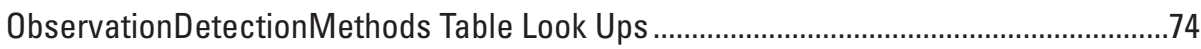

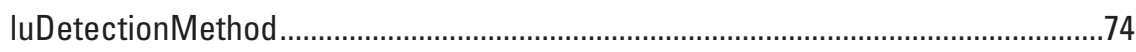

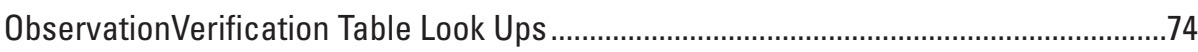

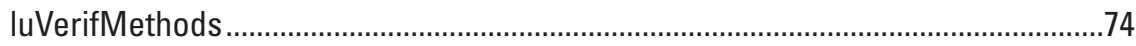

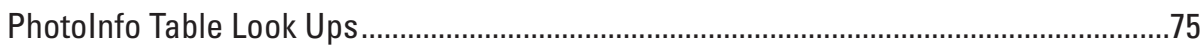

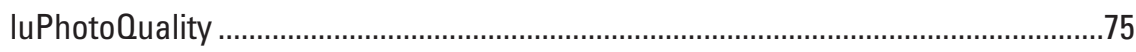

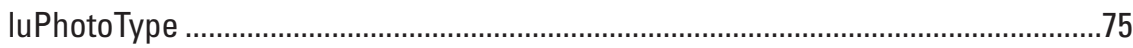

PlantEnhancement Table Look Ups ............................................................................

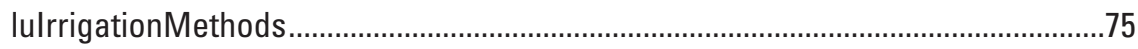

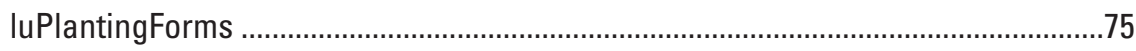


PlantHeirarchy Table Look Ups .................................................................................

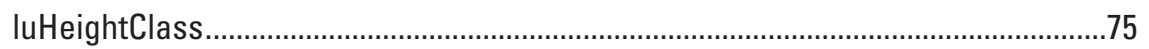

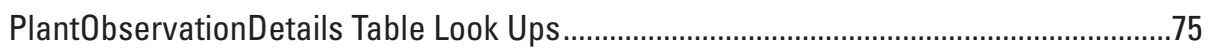

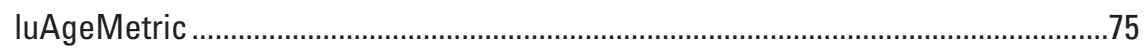

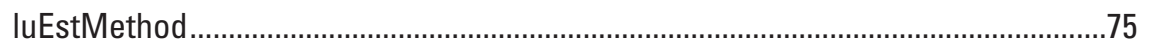

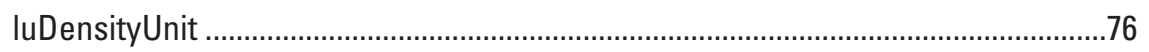

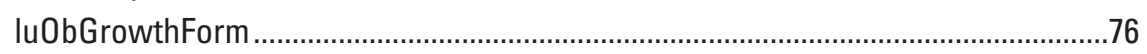

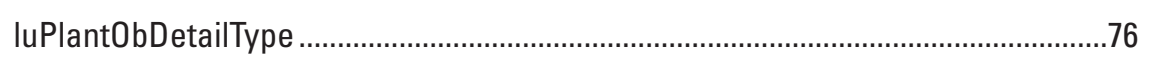

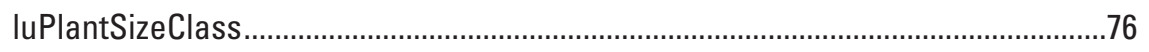

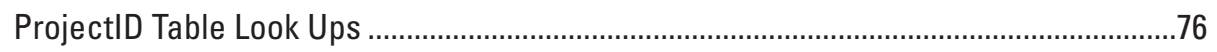

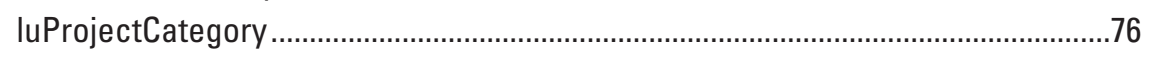

Refugia Table Look Ups.......................................................................................

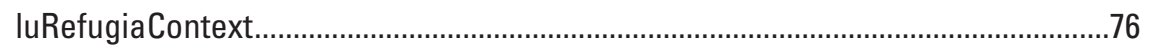

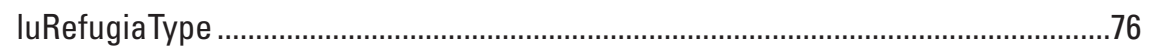

RoadsTrails Table Look Ups ...................................................................................

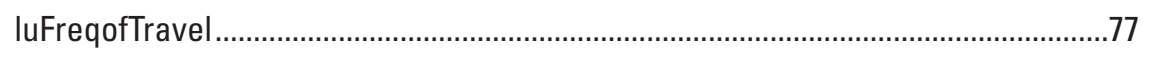

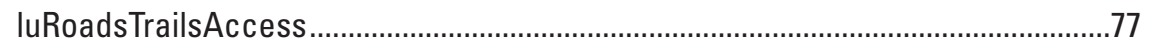

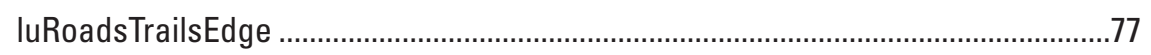

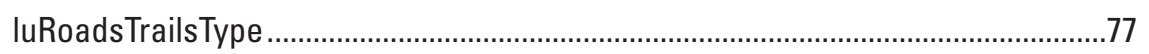

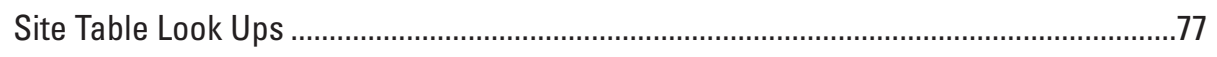

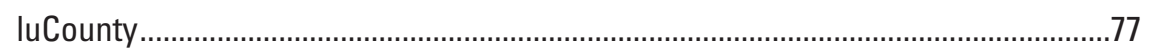

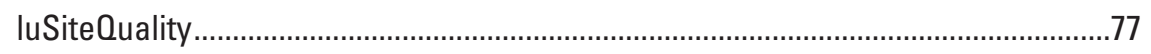

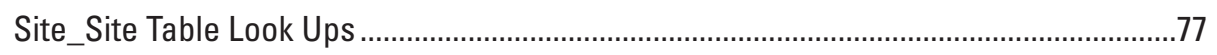

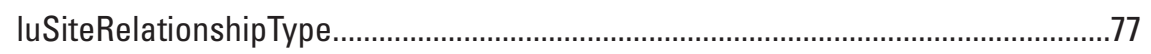

Site_SiteThreatType Table Look Ups .......................................................................

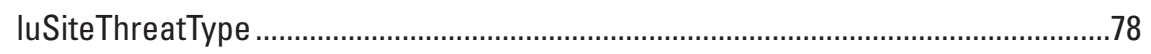

SoilPhysicalObservation Table Look Ups.................................................................

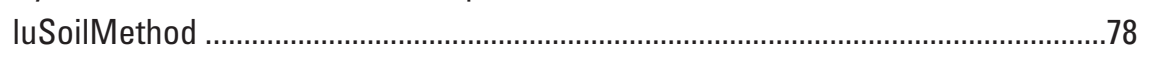

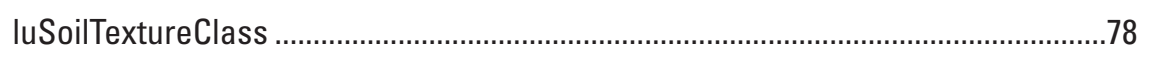

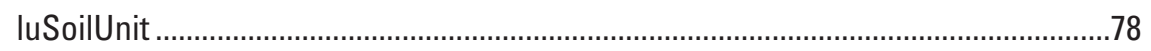

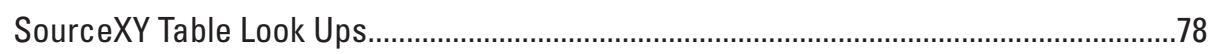

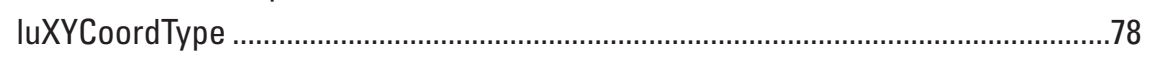

SpatialFeature_CLDB Table Look Ups ........................................................................

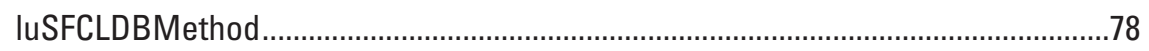

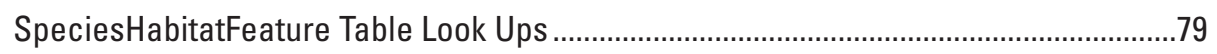

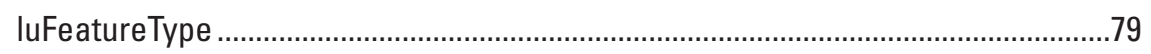

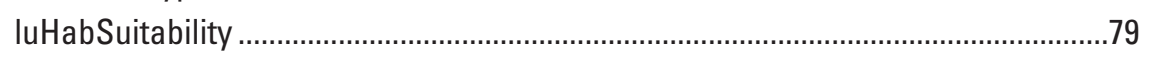

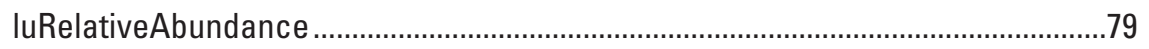

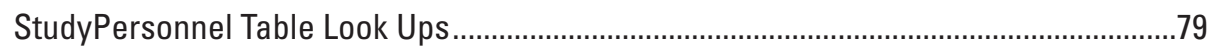

luAffiliateRole .....................................................................................................

SurveyDescription Table Look Ups ........................................................................

luStudyType................................................................................................

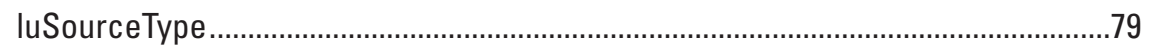


SurveyDescription_Site Table Look Ups........................................................................79

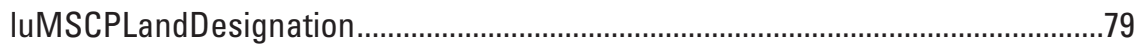

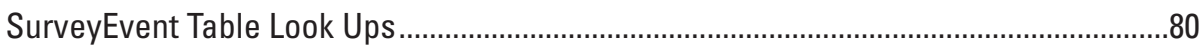

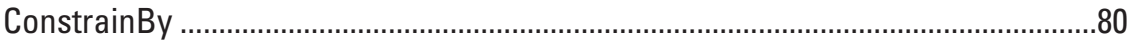

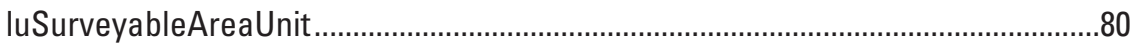

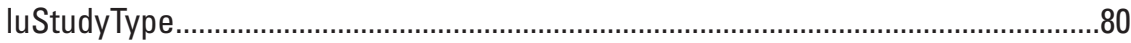

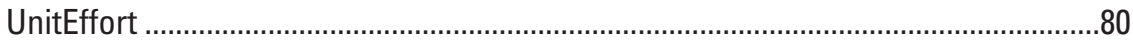

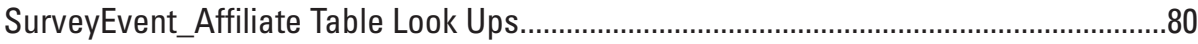

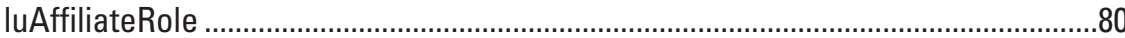

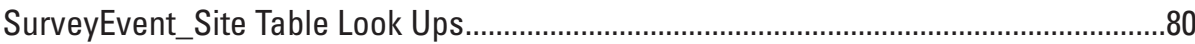

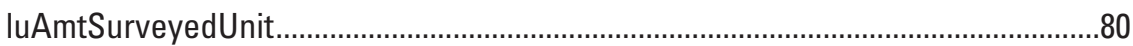

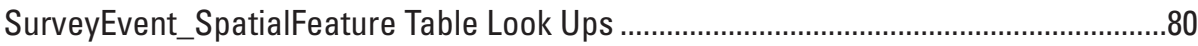

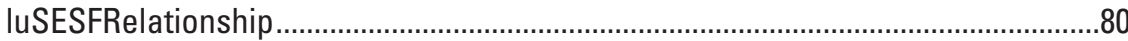

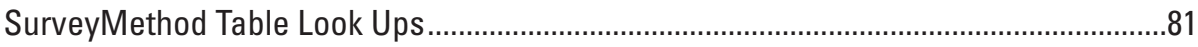

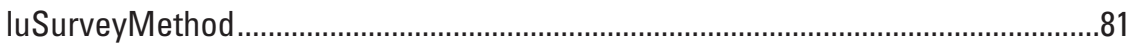

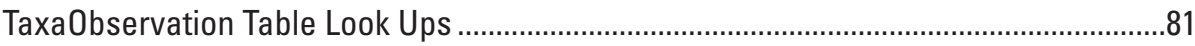

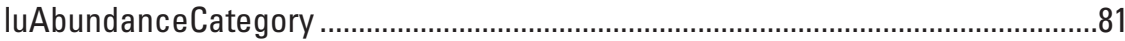

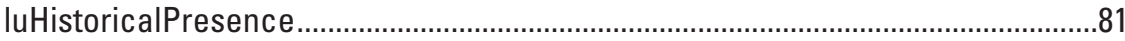

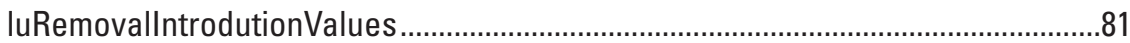

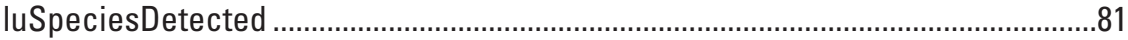

luTaxaRole

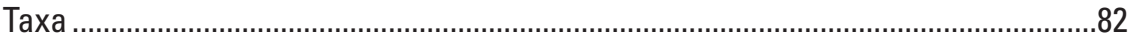

TaxaObservationDetails Table Look Ups....................................................................

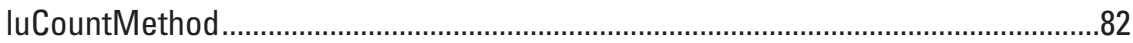

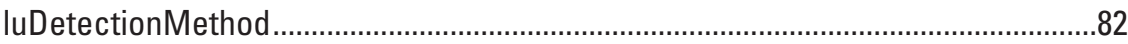

luGender

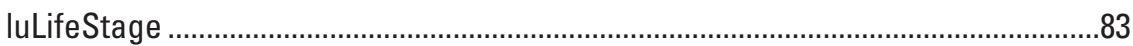

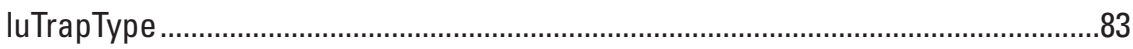

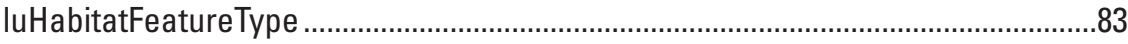

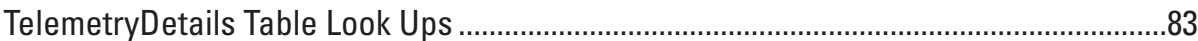

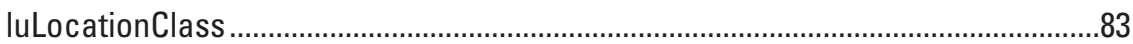

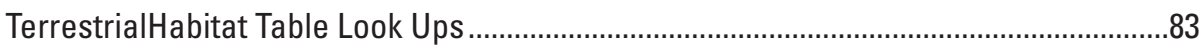

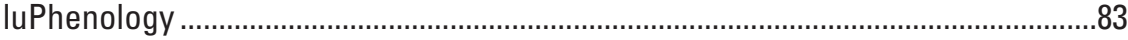

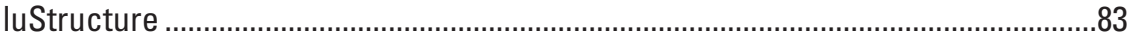

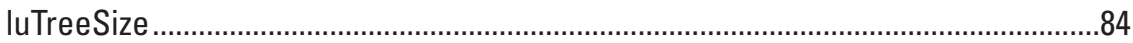

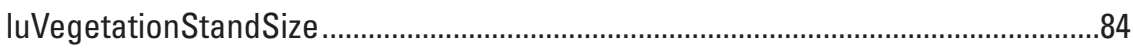

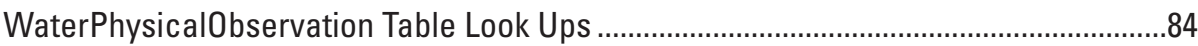

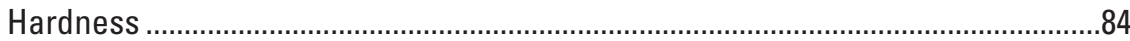

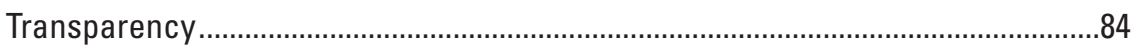

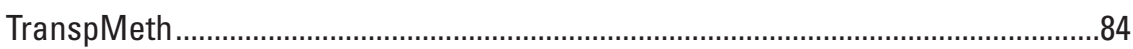

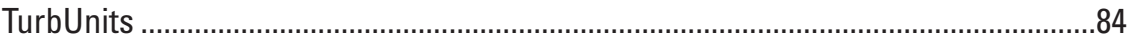

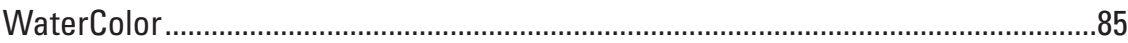


WeatherObservation Table Look Ups ..........................................................................85

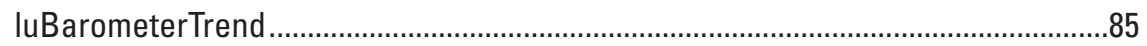

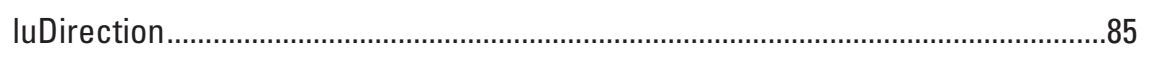

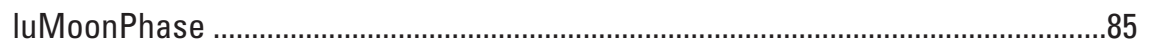

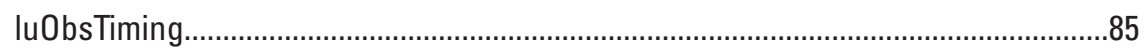

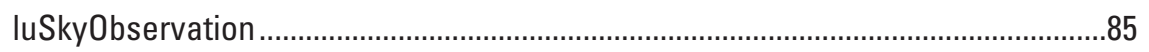

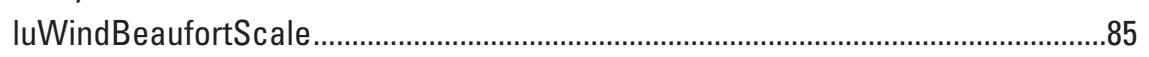

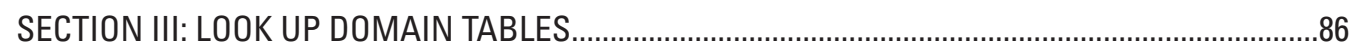

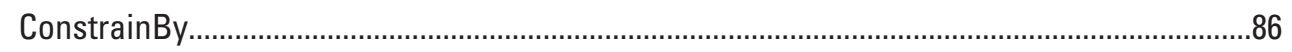

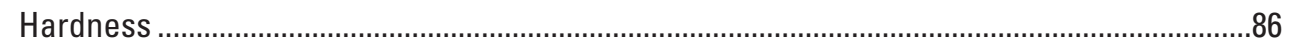

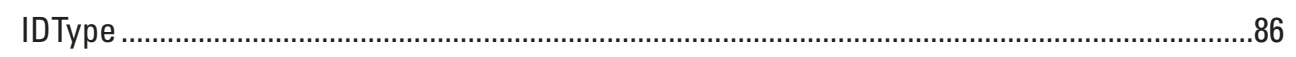

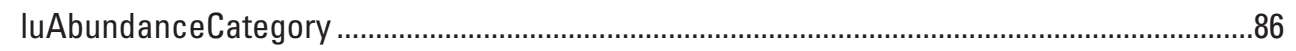

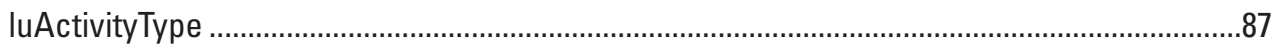

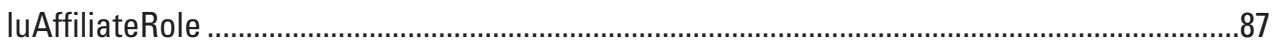

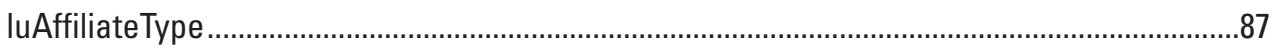

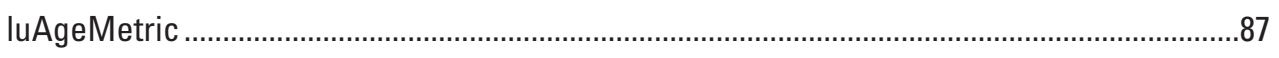

luAgePelage

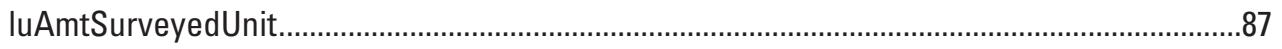

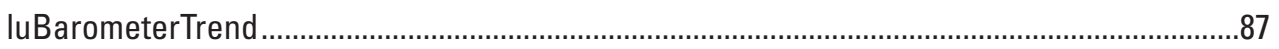

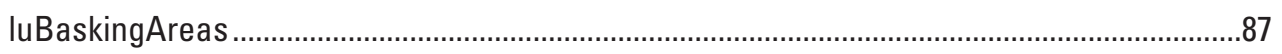

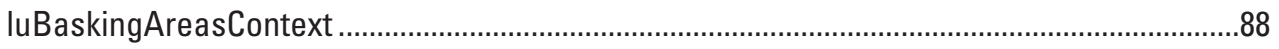

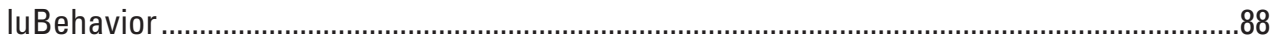

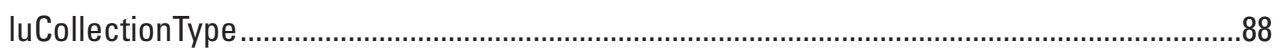

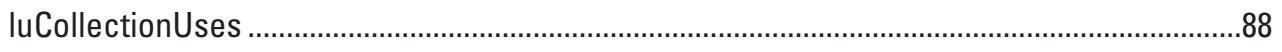

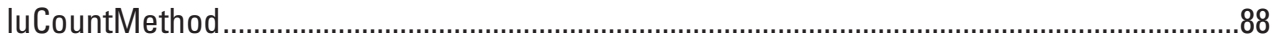

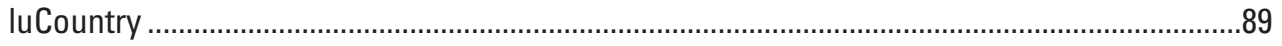

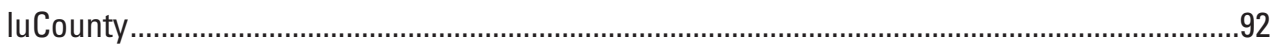

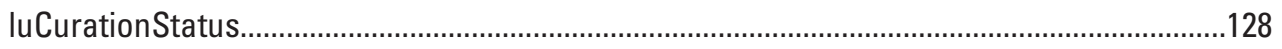

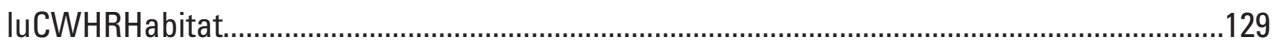

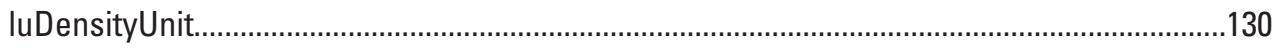

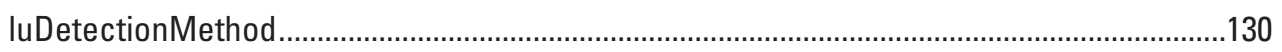

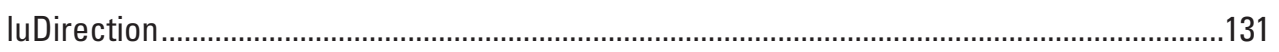

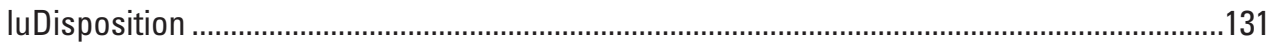

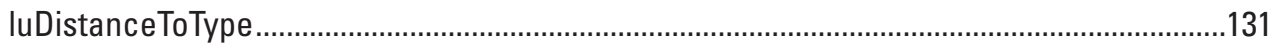

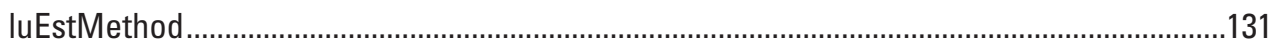

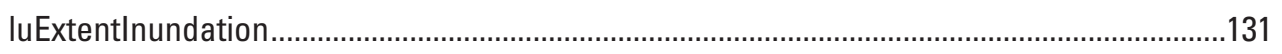

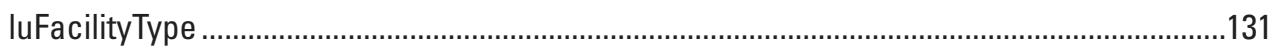

luFate 131

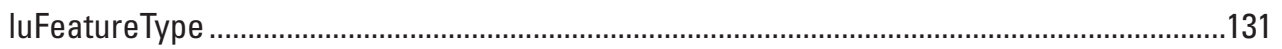

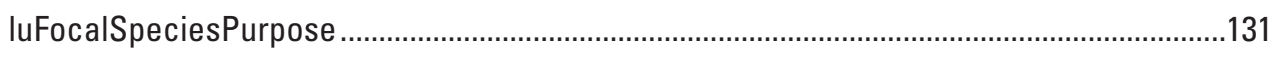

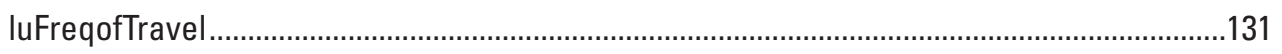

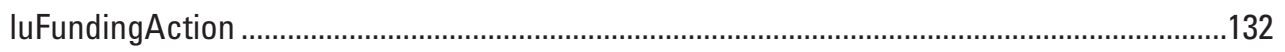

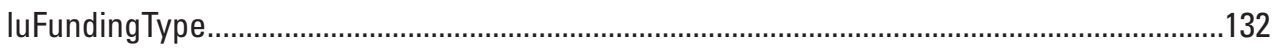

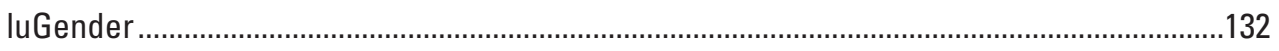

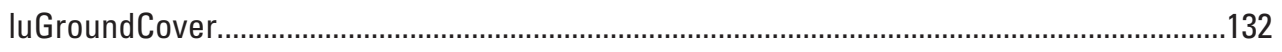




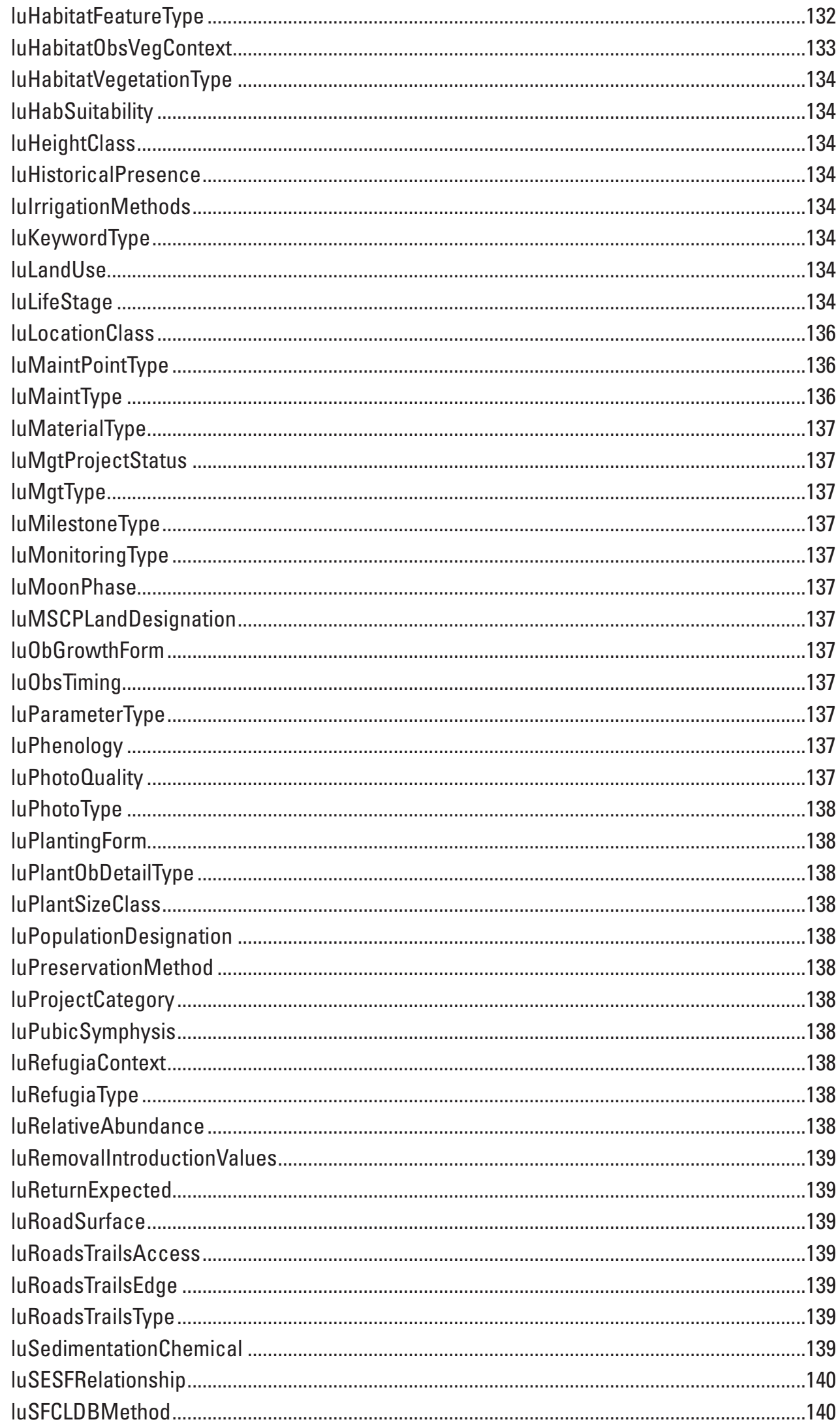




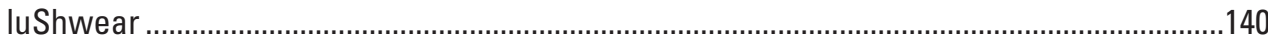

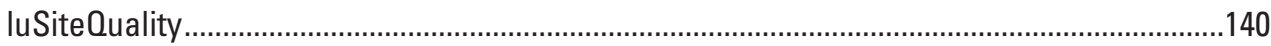

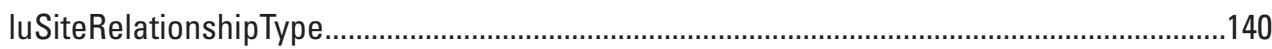

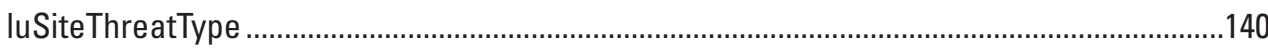

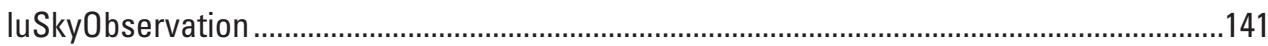

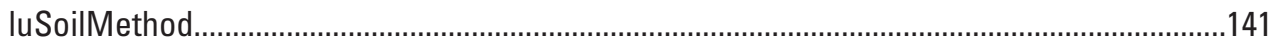

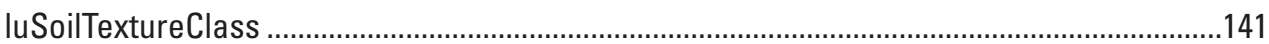

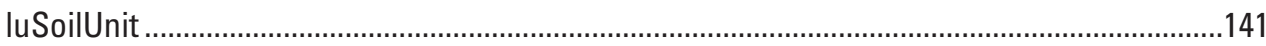

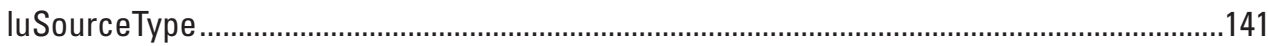

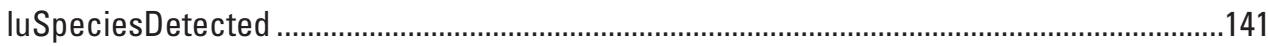

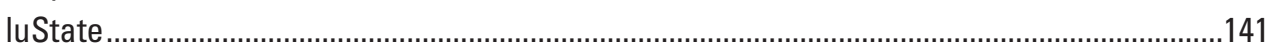

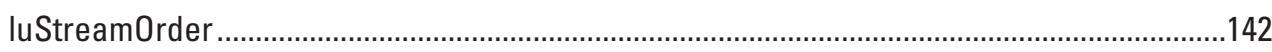

luStreamOrderMethodID ................................................................................................. 142

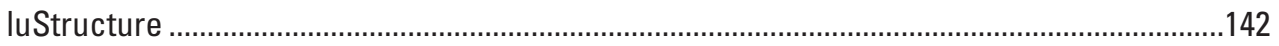

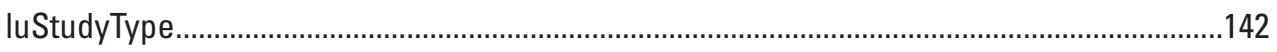

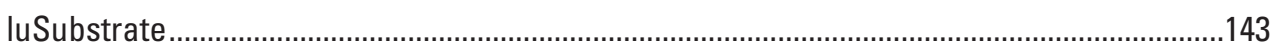

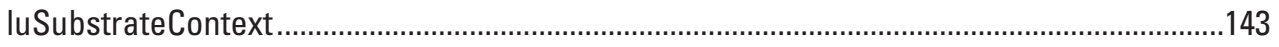

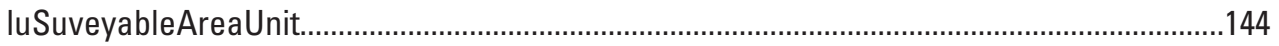

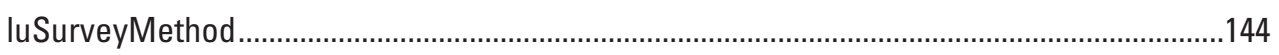

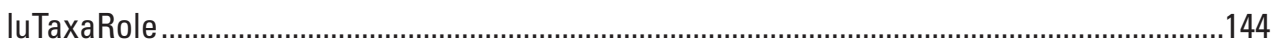

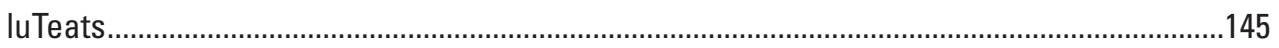

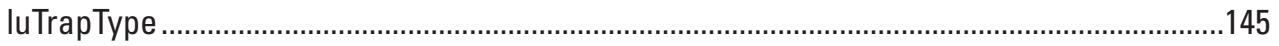

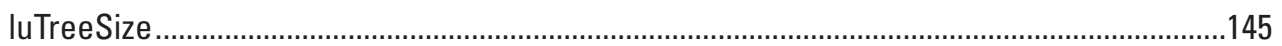

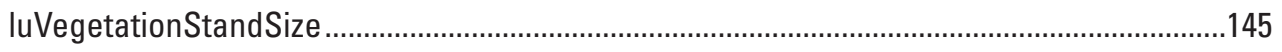

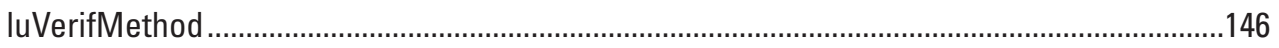

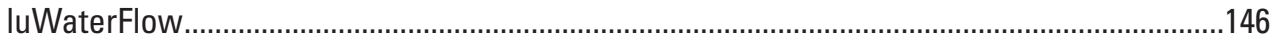

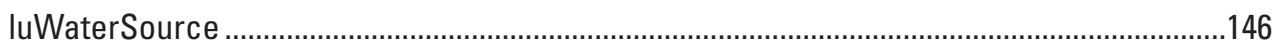

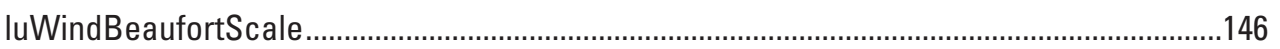

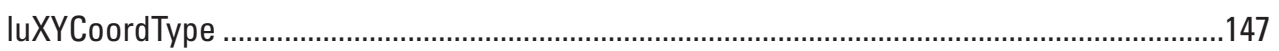

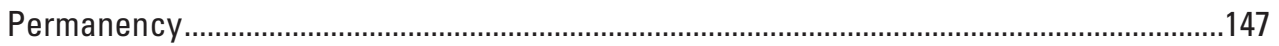

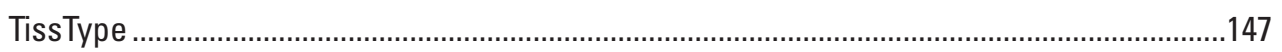

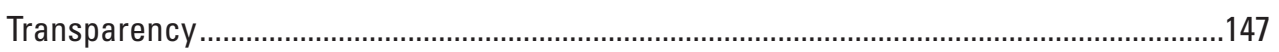

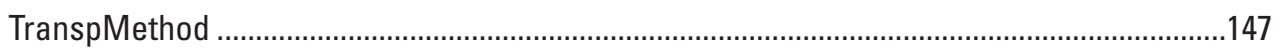

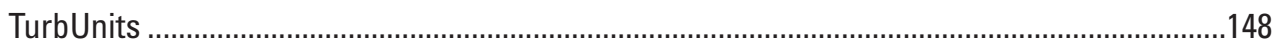

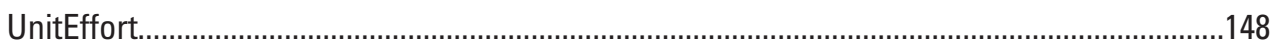

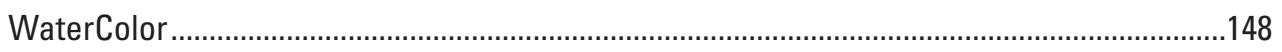

Appendix 1. Entity Relationship Diagram of All Database Tables.................................................149

Appendix 2. Entity Relationship Diagram of Tables Associated with Survey Events ...................149

Appendix 3. Entity Relationship Diagram of Tables Associated with Sites....................................149

Appendix 4. Entity Relationship Diagram of Tables Associated with Taxa Observations.............149

Appendix 5. Entity Relationship Diagram of Tables Associated with Habitat Observations .........149 


\section{Conversion Factors}

International System of Units to U.S. customary units

\begin{tabular}{lcl}
\hline \multicolumn{1}{c}{ Multiply } & By & \multicolumn{1}{c}{ To obtain } \\
\hline & Length & \\
\hline centimeter $(\mathrm{cm})$ & 0.3937 & inch (in.) \\
millimeter $(\mathrm{mm})$ & 0.03937 & inch (in.) \\
meter $(\mathrm{m})$ & 3.281 & foot (ft) \\
kilometer $(\mathrm{km})$ & 0.6214 & mile (mi) \\
kilometer $(\mathrm{km})$ & 0.5400 & mile, nautical (nmi) \\
meter $(\mathrm{m})$ & 1.094 & yard (yd) \\
\hline
\end{tabular}

\section{Supplemental Information}

Concentrations of chemical constituents in water are given in either milligrams per liter (mg/L) or micrograms per liter $(\mu \mathrm{g} / \mathrm{L})$.

\section{Abbreviations}

$\begin{array}{ll}\text { ARMI } & \text { Amphibian Research and Monitoring Initiative } \\ \text { ATV } & \text { All Terrain Vehicle } \\ \text { CDFW } & \text { California Department of Fish and Wildlife } \\ \text { CLDB } & \text { Conserved Lands Database } \\ \text { CWRH } & \text { California Wildlife Habitat Relationship } \\ \text { DAR } & \text { Distance angle rate } \\ \text { dB } & \text { decibels } \\ \text { DBH } & \text { diameter breast height } \\ \text { DBS } & \text { Database Management System } \\ \text { EtOH } & \text { Ethanol } \\ \text { FIPS } & \text { Federal Information Processing Standard } \\ \text { FTU } & \text { Formazin Turbidity Unit } \\ \text { GPS } & \text { Global Positioning System } \\ \text { Hg } & \text { Mercury } \\ \text { ITIS } & \text { Integrated Taxonomic Integration System }\end{array}$




$\begin{array}{ll}\text { kph } & \text { kilometers per hour } \\ \text { MCL } & \text { Maximum Carapace Length } \\ \mu \mathrm{g} / \mathrm{g} & \text { micrograms per gram } \\ \text { MRD } & \text { minimum redundancy } \\ \text { MSCP } & \text { Multiple Species Conservation Plan } \\ \text { MTX } & \text { Multi-Taxa database } \\ \text { NAAMP } & \text { North American Amphibian Monitoring Program } \\ \text { NPS } & \text { National Park Service } \\ \text { PDA } & \text { personal digital assistant } \\ \text { PIT } & \text { passive integrated transponder } \\ \text { NA } & \text { Not applicable } \\ \text { Ng/ul } & \text { nanograms per microliter } \\ \text { NTU } & \text { Nephelometric Turbidity Units } \\ \text { OAOC } & \text { Quality Assurance/ Ouality Control } \\ \text { SanDAG } & \text { San Diego Association of Governments } \\ \text { SDSU } & \text { San Diego State University } \\ \text { sec } & \text { seconds } \\ \text { T/F } & \text { True/False } \\ \text { USFWS } & \text { U.S. Fish and Wildlife Service } \\ \text { USGS } & \text { U.S. Geological Survey } \\ \text { UTM } & \text { Universal Transverse Mercator } \\ \text { VARCHAR } & \text { variable characters } \\ \text { VIE } & \text { Visible Implant Elastomer } \\ \text { Y/N } & \text { Yes/No } \\ & \end{array}$




\title{
Multi-Taxa Database Data Dictionary
}

\author{
By Elise Watson, Carlton J. Rochester, Chris W. Brown, Donn A. Holmes, Stacie A. Hathaway, and \\ Robert N. Fisher
}

\section{Abstract}

The conservation of biological resources relies on the successful management of ecological and physiological research data. The Western Ecological Research Center of the U.S. Geological Survey is working with researchers, land managers, and decision makers from non-government organizations and city, county, state, and federal resource agencies to develop data management methods. Access to the most current and applicable research data available in making sound decisions to conserve species diversity is foundational. We sought to accomplish several goals in developing the data management strategy used in the Multi-Taxa database. Data persistence and availability are primary goals of well-developed databases. By documenting and sharing the structure and definitions of Multi-Taxa database, we hope to further the successful management of these crucial data.

\section{Introduction}

\section{Background}

Successful management of biological resources relies on successful management of ecological and physiological research data. In California, the Western Ecological Research Center of the U.S. Geological Survey (USGS) is working with researchers and city, county, state, and federal resource agencies to develop strategies to supply data that meet management needs. We designed the Multi-Taxa database (MTX) described in this document to help meet the needs of resource managers in California and the western region while providing utility to researchers. We hope that others can benefit from understanding our database design.

Land managers and regulators rely on access to the most current and applicable research data available in making sound decisions to conserve species diversity. Integrated data management allows researchers, agencies, and consultants to share information regarding their independent research, inventory, and monitoring projects. We examined several data management strategies from local, regional, and federal partners to determine how to meet the needs of the managers. We consulted with federal and university researchers and statisticians to incorporate study design needs and capabilities and to ensure data of highest value were considered in our database strategy. Together, these concepts provided a basis on which a strategy could be developed.

We sought to accomplish several goals in developing the data management strategy used in the MTX database. One of the main goals was to develop a stringent database to provide a platform that could meet the needs of the managers and still provide flexibility for researchers. Peer reviewed and established protocols from wildlife agencies were thoroughly examined to develop data consistency. We provided for multiple data entry and recall tools to meet the demands of different study designs, including full digital data collection utilizing handheld computers with standardized forms, queries, and reports. Coupled with sound science, this strategy of integrated data management is of great benefit to the researchers and an invaluable tool to land managers.

Data persistence is a primary goal of well-developed relational databases. Having a robust relational database with well defined fields, relationships, and metadata allows the data to be useful long after the study for which it was originally collected has been completed. Relational databases can easily be managed with ever-evolving software. This capability, combined with the ability to quickly create backups and export data in multiple standard formats, such as comma or tab delimited text files, increases data persistence.

Databases like MTX can be used to export formatted data for many purposes. The Multi-Taxa database has been used to compile USGS data required by California scientific permits and to contribute to the National Wildlife Health Center lab results database that processes tissue sample data from the national Amphibian Research and Monitoring Initiative program (ARMI), which uses MTX for data management. The relational structure of these kinds of databases allows flexibility in developing reports and sharing data among researchers, and the permission system supports access restrictions on data until proper review has been conducted according to USGS procedures. 
Staff from several local and state agencies contributed to the early design of the MTX. The initial architecture of the MTX was influenced by the National Park Service's Inventory and Monitoring Program. Proposed database design, structure, and data fields were reviewed by staff from the California Department of Fish and Wildlife Biogeographic Data Branch, the U.S. Fish and Wildlife Service, and the National Park Service, as well as by U.S. Geological Survey biologists.

\section{Structure of the Database}

The MTX is a relational database, in which subject specific data tables are linked to each other using common fields ("foreign keys"). The MTX has several advantages for data storage. Data that are stored in the MTX are immediately accessible, given the proper access rights. The data can be reduced easily through simple query tools provided with many database applications ranging from shareware to industrial application software. The data can be queried repeatedly for multiple values across many fields at a very quick pace, without having to physically examine the entire dataset (either paper or spreadsheet) to look for representative values and types of data. By separating the spatial and temporal information and creating relationships between the categories of field data, collection errors can often be avoided. For example, errors such as recording an individual surveyor in multiple locations at the same time or twice entering the survey of a single location at a single time can be easily assessed or even not permitted. Relationships also clarify the associations among kinds of data. Particular data may pertain to a specific location at a specific time or they may have a larger spatial or temporal extent pertaining to multiple sites or surveys, which may be obscured when the data are in spreadsheet format. For example, many studies store data in single spreadsheets where relatively static site-specific data (for example, habitat type, slope, and aspect) are included and repeated for each survey. As well, survey specific covariates (for example, weather and water quality) can be repeated across locations or species observations in a survey. The structure of this database ensures that site covariate data will not be stored as survey covariates and vice versa.

Data and user security are integral to the structure of the database, with access permissions built into the database itself. In the case of the MTX, all access to data is managed through user accounts synchronized with the federal individual log-in system. User access is granted by by the data steward for each project. Users are never allowed direct access to a table; instead, specific data are selected, filtered, and presented through database coding, according to the design requirements of each data view. User access is linked to the data on a by-record-basis in order to make use of current and future applications to collect, review, and retrieve data.

\section{Purpose and Organization}

This document is intended to provide basic information on the structure and contents of the MTX database. Section I lists the principle data tables and their purposes. Tables are presented within their subject matter groups or "suites," such as Project Management related, Survey related, Site related, and so forth. Within each suite, the tables are presented in order of hierarchy of relatedness. Section II contains the definitions for all fields within the main tables (Part 1) and look-up tables (Part 2), which are presented alphabetically. Information on data types, field lengths, integer decimal places (Decimal Places), null requirements (Required Field), and Primary Key identification also is included with each field definition. Section III lists the domain values for each look-up table, which also are accessible by hyperlink functions in Section II. Hyperlinks allow the reader to jump directly to the associated look up table or field domain. In addition, the Navigation Window provides links to the main document sections as well as the ability to move forward or back in the document. Finally, in the appendix section, one can find a collection of diagrams that depict the relationships among all of the tables. These diagrams are commonly referred to as Entity Relationship Diagrams. 


\section{SECTION I: DATA TABLES1}

\section{Survey Data Table Suite}

\begin{tabular}{|c|c|c|}
\hline Table Name & Purpose & GoTo \\
\hline SurveyEvent & $\begin{array}{l}\text { The SurveyEvent table stores detailed information about the survey being conducted, including start and } \\
\text { end dates and times, surveyors, recorders, survey effort and constraints. This table is able to store multiple } \\
\text { observers in a way that they can be referenced in the species detection tables of the database. }\end{array}$ & E \\
\hline SurveyEvent_Site & $\begin{array}{l}\text { The SurveyEvent_Site table connects SurveyEvent records to Site records. This table also contains additional } \\
\text { data fields for survey-specific site information. }\end{array}$ & 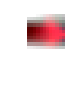 \\
\hline WeatherObservation & The WeatherObservation table stores data on weather conditions for the survey at a given point. & E \\
\hline
\end{tabular}

\section{Site Data Table Suite}

\begin{tabular}{|c|c|c|}
\hline Table Name & Purpose & GoTo \\
\hline Site & $\begin{array}{l}\text { The Site table contains data that describe the location of the site. It includes site name, subsites and sub- } \\
\text { subsites. The SiteID is carried down to the data entry tables below it, including those in other data suites. }\end{array}$ & E. \\
\hline Site_SiteThreatType & The Site_SiteThreatType table contains Site specific threat information. & = \\
\hline SpatialFeature & $\begin{array}{l}\text { The SpatialFeature table stores descriptive information about the location of an observation, such as waypoint } \\
\text { names or, if it is a revisit of a historical location, where a species was observed. }\end{array}$ & E \\
\hline SourceXY & $\begin{array}{l}\text { The SourceXY table stores data specific to the geographic location where data are being collected. This table } \\
\text { contains information on the coordinate types and collection methods. }\end{array}$ & = \\
\hline LandManagement & The LandManagement table contains information about the Land Managers for a site. & E \\
\hline SiteMaintenance & $\begin{array}{l}\text { The SiteMaintenance table stores data on maintenance needs and maintenance performed on the site. } \\
\text { Disprepair or ongoing repair at a site may influence survey data and may be responsible for data } \\
\text { abnormalities. This table records the dates of maintenance issues to account for this in the survey data. }\end{array}$ & = \\
\hline RoadsTrails & $\begin{array}{l}\text { The RoadsTrails table records the presence of roads and/or trails that exist on or near a given site. Associated } \\
\text { characteristics are also recorded for the given date. }\end{array}$ & E. \\
\hline
\end{tabular}

1This section describes only the tables relevant to data entry and does not include the additional tables that serve as lookup or administrative tables 


\section{Species Data Table Suite}

\begin{tabular}{|c|c|c|}
\hline Table Name & Purpose & GoTo \\
\hline TaxaObservation & $\begin{array}{l}\text { The TaxaObservation table stores general data about the species that has been detected, including if it } \\
\text { is a target species, and can store negative data for that species as well (that is, Species X was a target } \\
\text { species of the survey but was not detected). This table also stores observer/recorder information. }\end{array}$ & E. \\
\hline TaxaObservationDetails & $\begin{array}{l}\text { The TaxaObservation table stores specific data about the animal species that has been detected } \\
\text { including age class, gender and number of individuals of that category. }\end{array}$ & E \\
\hline IndivAnimals & $\begin{array}{l}\text { The IndivAnimal table stores data in reference to a specific animal during a specific sample event, such } \\
\text { as weight, length, mass and identification number/label. }\end{array}$ & $\mathrm{E}$ \\
\hline IndividualAnimalIdentifier & $\begin{array}{l}\text { The IndividualAnimalIdentifier table stores identification information for captured animals that are } \\
\text { tagged by some method (pit tag, toe clip, and so forth). Also noted is when an individual animal's ID } \\
\text { value or type has been changed or corrected. }\end{array}$ & 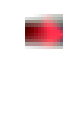 \\
\hline PlantObsevationDetails & $\begin{array}{l}\text { The PlantObservationDetails table stores specific data about a plant species that has been detected } \\
\text { including counts, areal estimates and physical characteristics. }\end{array}$ & $=$ \\
\hline Collection & $\begin{array}{l}\text { The Collection table stores data on physical items taken from the field, including whole or part(s) of } \\
\text { an animal or plant, soil and water samples. Quantity and storage location of the item(s) are recorded } \\
\text { here. The table allows for multiple collections to be associated with an individual animal. }\end{array}$ & 1 \\
\hline Collection_Affiliate & $\begin{array}{l}\text { The Collection_Affiliate table stores tracking information if a sample has been sent to a person outside } \\
\text { the USGS for identification or research purposes. }\end{array}$ & $=$ \\
\hline LabResults & The LabResults table stores information on lab results after samples have been processed. & = \\
\hline
\end{tabular}

\section{Habitat Data Table Suite}

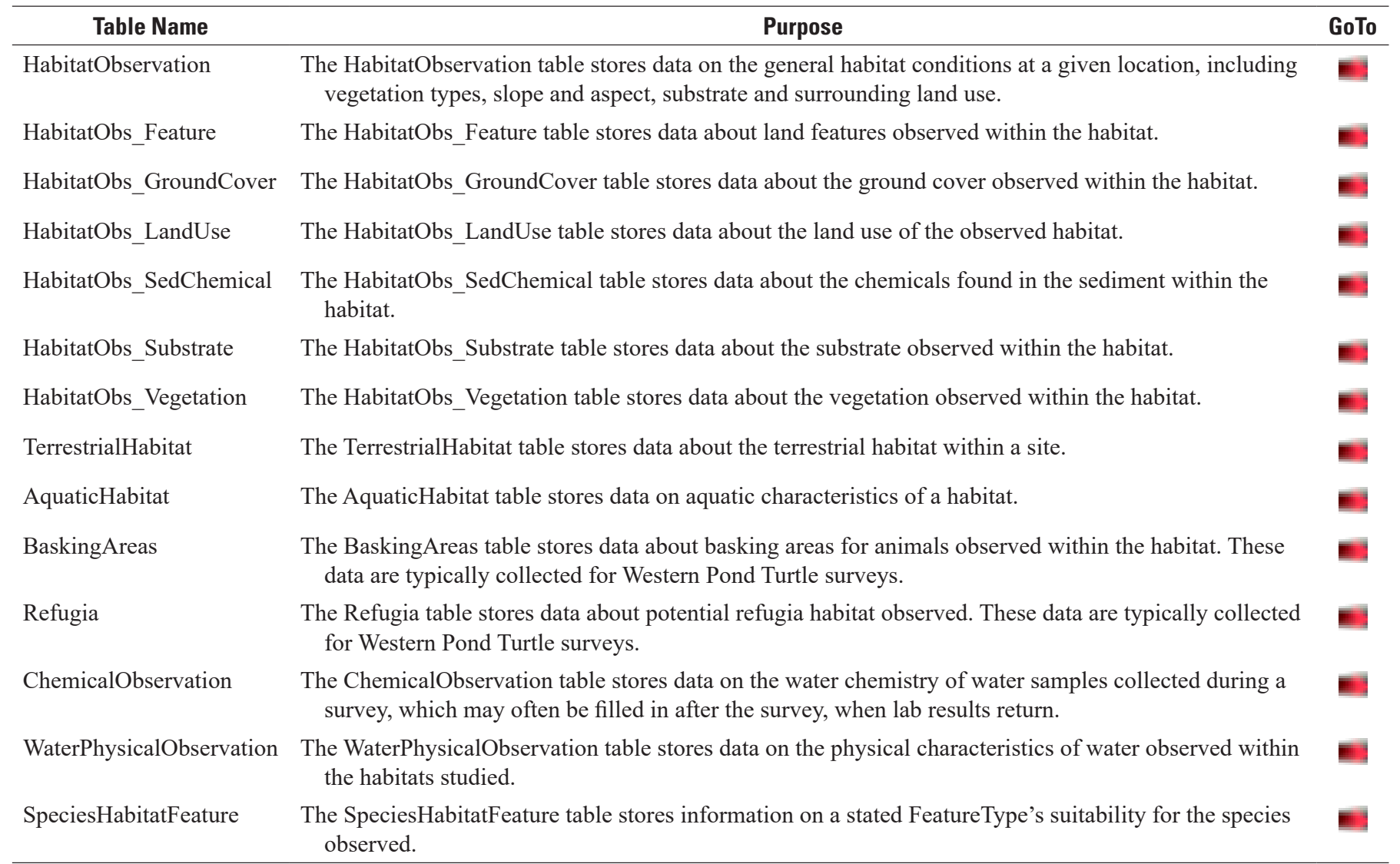




\section{SECTION II: FIELD DEFINITIONS}

PART 1. MAIN DATA TABLES

AbundanceEstimates Table

\begin{tabular}{|c|c|c|c|c|c|c|}
\hline Field Name & $\begin{array}{l}\text { Data } \\
\text { Type }\end{array}$ & $\begin{array}{c}\text { Field } \\
\text { Length }\end{array}$ & $\begin{array}{l}\text { Decimal } \\
\text { Places }\end{array}$ & $\begin{array}{l}\text { Required } \\
\text { Field }\end{array}$ & $\begin{array}{c}\text { Primary } \\
\text { Key }\end{array}$ & Definition \\
\hline 95ciHigh & decimal & 10 & 0 & $\mathrm{~N}$ & $\mathrm{~N}$ & $\begin{array}{l}\text { The upper bound of a } 95 \text { percent confidance interval around } \\
\text { the population estimate, should be greater than or equal to } \\
\text { 'pop_est'. }\end{array}$ \\
\hline 95ciLow & decimal & 10 & 0 & $\mathrm{~N}$ & $\mathrm{~N}$ & $\begin{array}{l}\text { The lower bound of a } 95 \text { percent confidance interval around the } \\
\text { population estimate, should be less than or equal to 'pop_est'. }\end{array}$ \\
\hline AEID & varchar & 50 & 0 & Y & $\mathrm{Y}$ & Abundance Estimates record identifier. \\
\hline CaptureProbability & decimal & 8 & 7 & $\mathrm{~N}$ & $\mathrm{~N}$ & An estimate of the individual capture probability (p-hat). \\
\hline CreatedBy & varchar & 64 & 0 & Y & $\mathrm{N}$ & Database username of person who created record. \\
\hline CreatedDate & datetime & 0 & 0 & $\mathrm{Y}$ & $\mathrm{N}$ & Date and time when record was created. \\
\hline EditDate & datetime & 0 & 0 & $\mathrm{~N}$ & $\mathrm{~N}$ & Date and time when record was most recently updated. \\
\hline EditedBy & varchar & 64 & 0 & $\mathrm{~N}$ & $\mathrm{~N}$ & Database username of person who most recently updated record. \\
\hline ESID & varchar & 50 & 0 & $\mathrm{Y}$ & $\mathrm{N}$ & Associated EstimateSeries record identifier. \\
\hline Gamma1 & decimal & 6 & 5 & $\mathrm{~N}$ & $\mathrm{~N}$ & $\begin{array}{l}\text { An estimate of the probability that an individual that is part } \\
\text { of the population was not available for sampling during the } \\
\text { sampling period (gamma prime). }\end{array}$ \\
\hline Gamma2 & decimal & 6 & 5 & $\mathrm{~N}$ & $\mathrm{~N}$ & $\begin{array}{l}\text { An estimate of the probability that an individual unavailable for } \\
\text { sampling in the previous year is still unavailable in the current } \\
\text { year (gamma double prime). }\end{array}$ \\
\hline Notes & varchar & 250 & 0 & $\mathrm{~N}$ & $\mathrm{~N}$ & Notes about the abundance estimate. \\
\hline NumberCaught & int & 0 & 0 & $\mathrm{~N}$ & $\mathrm{~N}$ & Number of animals included in estimates. \\
\hline PopEstError & decimal & 15 & 5 & $\mathrm{Y}$ & $\mathrm{N}$ & $\begin{array}{l}\text { An estimate of the standard error associated with the population } \\
\text { estimate. }\end{array}$ \\
\hline PopulationEstimate & decimal & 15 & 5 & $\mathrm{Y}$ & $\mathrm{N}$ & $\begin{array}{l}\text { An estimate of the number of individuals in the population } \\
\text { (N-hat). Use the superpopulation estimate if temporary } \\
\text { emigration has been estimated. }\end{array}$ \\
\hline Program & varchar & 50 & 0 & $\mathrm{~N}$ & $\mathrm{~N}$ & $\begin{array}{l}\text { The name of the software used to produce the parameter } \\
\text { estimate. }\end{array}$ \\
\hline QAQC_Note & varchar & 255 & 0 & $\mathrm{~N}$ & $\mathrm{~N}$ & Reason for change made to record during QAQC review. \\
\hline QAQC_Status & varchar & 20 & 0 & $\mathrm{Y}$ & $\mathrm{N}$ & Describes current stage of QAQC. \\
\hline RecaptureProbability & decimal & 8 & 7 & $\mathrm{~N}$ & $\mathrm{~N}$ & $\begin{array}{l}\text { An estimate of the probability of recapturing an individual that } \\
\text { has been previously captured(c-hat). }\end{array}$ \\
\hline Season & varchar & 15 & 0 & $\mathrm{~N}$ & $\mathrm{~N}$ & Season for which estimate was calculated. \\
\hline Version & varchar & 25 & 0 & $\mathrm{~N}$ & $\mathrm{~N}$ & Numers or letters identifying the version of the software. \\
\hline Year & varchar & 25 & 0 & $\mathrm{~N}$ & $\mathrm{~N}$ & The year(s) to which this estimate applies. \\
\hline
\end{tabular}




\section{Actions Table}

\begin{tabular}{|c|c|c|c|c|c|c|}
\hline Field Name & $\begin{array}{l}\text { Data } \\
\text { Type }\end{array}$ & $\begin{array}{c}\text { Field } \\
\text { Length }\end{array}$ & $\begin{array}{l}\text { Decimal } \\
\text { Places }\end{array}$ & $\begin{array}{l}\text { Required } \\
\text { Field }\end{array}$ & $\begin{array}{l}\text { Primary } \\
\text { Key }\end{array}$ & Definition \\
\hline ActionEndDate & datetime & 0 & 0 & $\mathrm{~N}$ & $\mathrm{~N}$ & End date of action. \\
\hline ActionName & varchar & 50 & 0 & $\mathrm{~N}$ & $\mathrm{~N}$ & Name of the action. \\
\hline CreatedBy & varchar & 64 & 0 & $\mathrm{Y}$ & $\mathrm{N}$ & Database username of person who created record. \\
\hline EditDate & datetime & 0 & 0 & $\mathrm{~N}$ & $\mathrm{~N}$ & Date and time when record was most recently updated. \\
\hline EditedBy & varchar & 64 & 0 & $\mathrm{~N}$ & $\mathrm{~N}$ & $\begin{array}{l}\text { Database username of person who most recently updated } \\
\text { record. }\end{array}$ \\
\hline InitiationDate & datetime & 0 & 0 & $\mathrm{~N}$ & $\mathrm{~N}$ & Date action initiated. \\
\hline Operative & int & 0 & 0 & $\mathrm{~N}$ & $\mathrm{~N}$ & Person or organization that carried out the action. \\
\hline ProtocolID & int & 0 & 0 & $\mathrm{~N}$ & $\mathrm{~N}$ & Associated Protocol record identifier. \\
\hline QAQC_Notes & varchar & 255 & 0 & $\mathrm{~N}$ & $\mathrm{~N}$ & Reason for change made to record during QAQC review. \\
\hline QAQC_Status & varchar & 20 & 0 & $\mathrm{Y}$ & $\mathrm{N}$ & Describes current stage of QAQC. \\
\hline TargetHabitatType & varchar & 3 & 0 & $\mathrm{~N}$ & $\mathrm{~N}$ & $\begin{array}{l}\text { Habitat, based on updated vegetation classification system, } \\
\text { which action pertains to. See luCWHRHabitat. }\end{array}$ \\
\hline TargetSpecies & varchar & 25 & 0 & $\mathrm{~N}$ & $\mathrm{~N}$ & $\begin{array}{l}\text { Integrated Taxonomic Integration System (ITIS) taxonomic } \\
\text { serial number or assigned identifier of plant, animal, } \\
\text { or vegetation community that action pertains to. } \\
\text { See http://www.itis.gov/ for plant or animal species } \\
\text { information. }\end{array}$ \\
\hline
\end{tabular}

Affiliate Table

\begin{tabular}{lcccccl}
\hline \multicolumn{1}{c}{ Field Name } & $\begin{array}{c}\text { Data } \\
\text { Type }\end{array}$ & $\begin{array}{c}\text { Field } \\
\text { Length }\end{array}$ & $\begin{array}{c}\text { Decimal } \\
\text { Places }\end{array}$ & $\begin{array}{c}\text { Required } \\
\text { Field }\end{array}$ & $\begin{array}{c}\text { Primary } \\
\text { Key }\end{array}$ & Definition \\
\hline Address1 & varchar & 100 & 0 & $\mathrm{~N}$ & $\mathrm{~N}$ & Affiliate's first line of address. \\
Address2 & varchar & 100 & 0 & $\mathrm{~N}$ & $\mathrm{~N}$ & Affiliate's second line of address. \\
Affiliate_Initials & varchar & 10 & 0 & $\mathrm{~N}$ & $\mathrm{~N}$ & Affiliate's initials. \\
AffiliateID & int & 0 & 0 & $\mathrm{Y}$ & $\mathrm{Y}$ & Affiliate record identifier. \\
AffiliateRecord-Validated & bit & 0 & 0 & $\mathrm{~N}$ & $\mathrm{~N}$ & Indicates if the Affiliate record has been validated. \\
AffiliateTypeID & int & 0 & 0 & $\mathrm{Y}$ & $\mathrm{N}$ & Type of organization affiliate belongs to. See \\
& & & & & & luAffiliateType. \\
CDFGPermitNum & varchar & 25 & 0 & $\mathrm{~N}$ & $\mathrm{~N}$ & CDFW (formerly CDFG) Permit number issued to affiliate. \\
CellPhone & varchar & 20 & 0 & $\mathrm{~N}$ & $\mathrm{~N}$ & Affiliate's cell phone number. \\
City & varchar & 50 & 0 & $\mathrm{~N}$ & $\mathrm{~N}$ & Affiliate's address city. \\
CountryCode & char & 2 & 0 & $\mathrm{~N}$ & $\mathrm{~N}$ & Affiliate's address country abbreviation. See luCountry.
\end{tabular}


Affiliate Table-Continued

\begin{tabular}{|c|c|c|c|c|c|c|}
\hline Field Name & $\begin{array}{l}\text { Data } \\
\text { Type }\end{array}$ & $\begin{array}{l}\text { Field } \\
\text { Length }\end{array}$ & $\begin{array}{c}\text { Decimal } \\
\text { Places }\end{array}$ & $\begin{array}{l}\text { Required } \\
\text { Field }\end{array}$ & $\begin{array}{c}\text { Primary } \\
\text { Key }\end{array}$ & Definition \\
\hline Created & datetime & 0 & 0 & $\mathrm{~N}$ & $\mathrm{~N}$ & Pendragon PDA field, for PDA compatibility. \\
\hline CreatedDate & datetime & 0 & 0 & Y & $\mathrm{N}$ & Date and time when record was created. \\
\hline EditDate & datetime & 0 & 0 & $\mathrm{~N}$ & $\mathrm{~N}$ & Date and time when record was most recently updated. \\
\hline EMail & varchar & 320 & 0 & $\mathrm{~N}$ & $\mathrm{~N}$ & Affiliate's email address. \\
\hline Extension & varchar & 4 & 0 & $\mathrm{~N}$ & $\mathrm{~N}$ & Affiliate's phone number extension. \\
\hline Fax & varchar & 20 & 0 & $\mathrm{~N}$ & $\mathrm{~N}$ & Affiliate's fax number. \\
\hline FirstName & varchar & 50 & 0 & $\mathrm{~N}$ & $\mathrm{~N}$ & Affiliate's first name. \\
\hline LoginName & varchar & 20 & 0 & $\mathrm{~N}$ & $\mathrm{~N}$ & Affiliate's login name for MTX. \\
\hline MiddleInit & varchar & 1 & 0 & $\mathrm{~N}$ & $\mathrm{~N}$ & Affiliate's middle initial. \\
\hline Organization & varchar & 100 & 0 & $\mathrm{~N}$ & $\mathrm{~N}$ & Affiliate's organization. \\
\hline Phone & varchar & 20 & 0 & $\mathrm{~N}$ & $\mathrm{~N}$ & Affiliate's phone number. \\
\hline PostalCode & varchar & 50 & 0 & $\mathrm{~N}$ & $\mathrm{~N}$ & Affiliate's address postal zip code. \\
\hline RecordID & int & 0 & 0 & $\mathrm{~N}$ & $\mathrm{~N}$ & Pendragon PDA field, for PDA compatibility. \\
\hline SourceOfRecord & varchar & 50 & 0 & $\mathrm{~N}$ & $\mathrm{~N}$ & Source of the Affiiate record information. \\
\hline StateCode & char & 2 & 0 & $\mathrm{~N}$ & $\mathrm{~N}$ & Affiliate's address state abbreviation. See luState. \\
\hline StatusCode & char & 1 & 0 & $\mathrm{Y}$ & $\mathrm{N}$ & Current status of affiliate, $\mathrm{A}=$ Active; $\mathrm{I}=$ Inactive. \\
\hline
\end{tabular}

\section{Affiliate_Affiliate Table}

\begin{tabular}{lcccccl}
\hline \multicolumn{1}{c}{ Field Name } & $\begin{array}{c}\text { Data } \\
\text { Type }\end{array}$ & $\begin{array}{c}\text { Field } \\
\text { Length }\end{array}$ & $\begin{array}{c}\text { Decimal } \\
\text { Places }\end{array}$ & $\begin{array}{c}\text { Required } \\
\text { Field }\end{array}$ & $\begin{array}{c}\text { Primary } \\
\text { Key }\end{array}$ & Definition \\
\hline AffiliateAffiliateID & int & 0 & 0 & $\mathrm{Y}$ & $\mathrm{Y}$ & Affiliate_Affiliate record identifier. \\
AffiliateID & int & 0 & 0 & $\mathrm{Y}$ & $\mathrm{Y}$ & Associated Affiliate record identifier. \\
CreatedBy & varchar & 64 & 0 & $\mathrm{Y}$ & $\mathrm{N}$ & Database username of person who created record. \\
CreatedDate & datetime & 0 & 0 & $\mathrm{Y}$ & $\mathrm{N}$ & Date and time when record was created. \\
EditDate & datetime & 0 & 0 & $\mathrm{~N}$ & $\mathrm{~N}$ & Date and time when record was most recently updated. \\
EditedBy & varchar & 64 & 0 & $\mathrm{~N}$ & $\mathrm{~N}$ & Database username of person who most recently updated record. \\
MailToAffiliateID & int & 0 & 0 & $\mathrm{Y}$ & $\mathrm{Y}$ & Affiliate record identifier of person mailings should be directed to. \\
ParentAffiliateID & int & 0 & 0 & $\mathrm{Y}$ & $\mathrm{Y}$ & Affiliate record identifier of parent affiliate (such as organization \\
& & & & & & that person belongs to). \\
StatusCode & varchar & 2 & 0 & $\mathrm{~N}$ & $\mathrm{~N}$ & Current status of affiliate, A = Active, I= Inactive.
\end{tabular}


Affiliate_Communication Table

\begin{tabular}{lcccccl}
\hline \multicolumn{1}{c}{ Field Name } & $\begin{array}{c}\text { Data } \\
\text { Type }\end{array}$ & $\begin{array}{c}\text { Field } \\
\text { Length }\end{array}$ & $\begin{array}{c}\text { Decimal } \\
\text { Places }\end{array}$ & $\begin{array}{c}\text { Required } \\
\text { Field }\end{array}$ & $\begin{array}{c}\text { Primary } \\
\text { Key }\end{array}$ & Definition \\
\hline AffiliateID & int & 0 & 0 & $\mathrm{Y}$ & $\mathrm{Y}$ & Affiliate record identifier. \\
CommunicationID & bigint & 19 & 0 & $\mathrm{Y}$ & $\mathrm{Y}$ & Communication record identifier. \\
\hline
\end{tabular}

Aquatic Habitat Table

\begin{tabular}{|c|c|c|c|c|c|c|}
\hline Field Name & $\begin{array}{l}\text { Data } \\
\text { Type }\end{array}$ & $\begin{array}{c}\text { Field } \\
\text { Length }\end{array}$ & $\begin{array}{l}\text { Decimal } \\
\text { Places }\end{array}$ & $\begin{array}{l}\text { Required } \\
\text { Field }\end{array}$ & $\begin{array}{l}\text { Primary } \\
\text { Key }\end{array}$ & Definition \\
\hline AquaticHabitatID & varchar & 50 & 0 & $\mathrm{Y}$ & $\mathrm{Y}$ & AquaticHabitat record identifier. \\
\hline Created & datetime & 0 & 0 & $\mathrm{~N}$ & $\mathrm{~N}$ & Pendragon PDA field, for PDA compatibility. \\
\hline CreatedBy & varchar & 64 & 0 & Y & $\mathrm{N}$ & Database username of person who created record. \\
\hline EditDate & datetime & 0 & 0 & $\mathrm{~N}$ & $\mathrm{~N}$ & Date and time when record was most recently updated. \\
\hline EditedBy & varchar & 64 & 0 & $\mathrm{~N}$ & $\mathrm{~N}$ & $\begin{array}{l}\text { Database username of person who most recently updated } \\
\text { record. }\end{array}$ \\
\hline ExtentInundationID & int & 0 & 0 & $\mathrm{~N}$ & $\mathrm{~N}$ & $\begin{array}{l}\text { Percent range of site covered in water. See luExtentInundation } \\
\text { table. }\end{array}$ \\
\hline FloodProneWidth & decimal & 9 & 2 & $\mathrm{~N}$ & $\mathrm{~N}$ & $\begin{array}{l}\text { The width measured at an elevation which is determined at } \\
\text { twice the maximum bankfull depth ( } 1 \text { to } 50 \text { year flood); } \\
\text { measured in meters. }\end{array}$ \\
\hline FlowDirectionCode & varchar & 3 & 0 & $\mathrm{~N}$ & $\mathrm{~N}$ & Abbreviation of direction of water flow. \\
\hline HabitatObservationID & varchar & 50 & 0 & $\mathrm{~N}$ & $\mathrm{~N}$ & Associated HabitatObservation record identifier. \\
\hline IsFishBarrierPresent & int & 0 & 0 & $\mathrm{~N}$ & $\mathrm{~N}$ & Y/N. If Fish Barrier present in area surveyed. \\
\hline IsFishBarrierStart & int & 0 & 0 & $\mathrm{~N}$ & $\mathrm{~N}$ & Y/N. If Fish Barrier starts in area surveyed. \\
\hline Notes & varchar & 250 & 0 & $\mathrm{~N}$ & $\mathrm{~N}$ & Notes about the aquatic habitat observation. \\
\hline Canopy & varchar & 10 & 0 & $\mathrm{~N}$ & $\mathrm{~N}$ & $\begin{array}{l}\text { Percentage of area of the stream covered by the topmost } \\
\text { vegetative cover. Can be calculated by estimating the average } \\
\text { percent of the sky visible between } 45 \text { degree upward angles } \\
\text { of the bank. }\end{array}$ \\
\hline $\begin{array}{l}\text { PercentReachWith } \\
\text { DeepPool }\end{array}$ & varchar & 10 & 0 & $\mathrm{~N}$ & $\mathrm{~N}$ & Percent-range of reach with deep pooling water. \\
\hline $\begin{array}{l}\text { PercentReachWith } \\
\text { MedPool }\end{array}$ & varchar & 10 & 0 & $\mathrm{~N}$ & $\mathrm{~N}$ & Percent-range of reach with medium pooling water. \\
\hline $\begin{array}{l}\text { PercentReachWith } \\
\quad \text { Riffles }\end{array}$ & varchar & 10 & 0 & $\mathrm{~N}$ & $\mathrm{~N}$ & Percent-range of reach with riffles visible in water. \\
\hline
\end{tabular}




\begin{tabular}{|c|c|c|c|c|c|c|}
\hline Field Name & $\begin{array}{l}\text { Data } \\
\text { Type }\end{array}$ & $\begin{array}{l}\text { Field } \\
\text { Length }\end{array}$ & $\begin{array}{l}\text { Decimal } \\
\text { Places }\end{array}$ & $\begin{array}{l}\text { Required } \\
\text { Field }\end{array}$ & $\begin{array}{l}\text { Primary } \\
\text { Key }\end{array}$ & Definition \\
\hline $\begin{array}{l}\text { PercentReachWith } \\
\quad \text { Runs }\end{array}$ & varchar & 10 & 0 & $\mathrm{~N}$ & $\mathrm{~N}$ & Percent-range of reach with running water. \\
\hline $\begin{array}{l}\text { PercentReachWith } \\
\text { ShallowPool }\end{array}$ & varchar & 10 & 0 & $\mathrm{~N}$ & $\mathrm{~N}$ & Percent-range of reach with shallow pooling water. \\
\hline $\begin{array}{l}\text { PercentWetLength } \\
\text { OfSurvey }\end{array}$ & varchar & 10 & 0 & $\mathrm{~N}$ & $\mathrm{~N}$ & Percent-range for length of survey that is wet. \\
\hline QAQC_Note & varchar & 255 & 0 & $\mathrm{~N}$ & $\mathrm{~N}$ & Reason for change made to record during QAQC review. \\
\hline QAQC_Status & varchar & 20 & 0 & $\mathrm{Y}$ & $\mathrm{N}$ & Describes current stage of QAQC. \\
\hline RecordID & int & 0 & 0 & $\mathrm{~N}$ & $\mathrm{~N}$ & Pendragon PDA field, for PDA compatibility. \\
\hline StreamOrderID & int & 0 & 0 & $\mathrm{~N}$ & $\mathrm{~N}$ & $\begin{array}{l}\text { Stream size based on a hierarchy of tributaries. See } \\
\text { luStreamOrder table. }\end{array}$ \\
\hline StreamSlopeDegrees & decimal & 10 & 4 & $\mathrm{~N}$ & $\mathrm{~N}$ & Slope of stream in degrees. \\
\hline UnitID & int & 0 & 0 & $\mathrm{~N}$ & $\mathrm{~N}$ & Pendragon PDA field, for PDA compatibility. \\
\hline UserName & varchar & 64 & 0 & $\mathrm{~N}$ & $\mathrm{~N}$ & Pendragon PDA field, for PDA compatibility. \\
\hline WaterAerialExtent & decimal & 10 & 4 & $\mathrm{~N}$ & $\mathrm{~N}$ & Extent of area of water feature in square meters. \\
\hline WaterAverageDepth & decimal & 10 & 4 & $\mathrm{~N}$ & $\mathrm{~N}$ & Average depth of water feature in meters. \\
\hline WaterAverageLength & decimal & 10 & 4 & $\mathrm{~N}$ & $\mathrm{~N}$ & Average length of water feature in meters. \\
\hline WaterAverageWidth & decimal & 10 & 4 & $\mathrm{~N}$ & $\mathrm{~N}$ & Average width of water feature in meters. \\
\hline WaterDepth & decimal & 10 & 4 & $\mathrm{~N}$ & $\mathrm{~N}$ & Water depth at the point in meters. \\
\hline WaterFlowID & int & 0 & 0 & $\mathrm{~N}$ & $\mathrm{~N}$ & Flow rate of the water. See luWaterFlow table. \\
\hline WaterLength & decimal & 10 & 4 & $\mathrm{~N}$ & $\mathrm{~N}$ & Length of water feature in meters. \\
\hline WaterMaxDepth & decimal & 10 & 4 & $\mathrm{~N}$ & $\mathrm{~N}$ & Maximum depth of water feature in meters. \\
\hline WaterMaxLength & decimal & 10 & 4 & $\mathrm{~N}$ & $\mathrm{~N}$ & Maximum length of water feature in meters. \\
\hline WaterMaxWidth & decimal & 10 & 4 & $\mathrm{~N}$ & $\mathrm{~N}$ & Maximum width of water feature in meters. \\
\hline WaterSourceCode & varchar & 2 & 0 & $\mathrm{~N}$ & $\mathrm{~N}$ & Origin of water body. See luWaterSource table. \\
\hline WaterVelocity & decimal & 10 & 4 & $\mathrm{~N}$ & $\mathrm{~N}$ & Velocity of water in meters/second. \\
\hline WaterWidth & decimal & 10 & 4 & $\mathrm{~N}$ & $\mathrm{~N}$ & Width of water feature in meters. \\
\hline
\end{tabular}

\section{Basking Areas Table}

\begin{tabular}{|c|c|c|c|c|c|c|}
\hline Field Name & $\begin{array}{l}\text { Data } \\
\text { Type }\end{array}$ & $\begin{array}{l}\text { Field } \\
\text { Length }\end{array}$ & $\begin{array}{l}\text { Decimal } \\
\text { Places }\end{array}$ & $\begin{array}{l}\text { Required } \\
\text { Field }\end{array}$ & $\begin{array}{l}\text { Primary } \\
\text { Key }\end{array}$ & Definition \\
\hline BaskingArea & varchar & 20 & 0 & $\mathrm{~N}$ & $\mathrm{~N}$ & $\begin{array}{l}\text { Area above the surface of the water where sunny space } \\
\text { is available for animals to rest and sun themselves (for } \\
\text { example, rocks, sunny banks). See luBaskingAreas table. }\end{array}$ \\
\hline BaskingAreasID & varchar & 50 & 0 & $\mathrm{Y}$ & $\mathrm{Y}$ & BaskingAreas record identifier. \\
\hline Context & varchar & 20 & 0 & $\mathrm{~N}$ & $\mathrm{~N}$ & $\begin{array}{l}\text { Area within habitat where basking area is observed. See } \\
\text { luBaskingAreasContext table. }\end{array}$ \\
\hline Created & datetime & 0 & 0 & $\mathrm{~N}$ & $\mathrm{~N}$ & Pendragon PDA field, for PDA compatibility. \\
\hline CreatedBy & varchar & 64 & 0 & Y & $\mathrm{N}$ & Database username of person who created record. \\
\hline CreatedDate & datetime & 0 & 0 & $\mathrm{Y}$ & $\mathrm{N}$ & Date and time when record was created. \\
\hline EditDate & datetime & 0 & 0 & $\mathrm{~N}$ & $\mathrm{~N}$ & Date and time when record was most recently updated. \\
\hline EditedBy & varchar & 64 & 0 & $\mathrm{~N}$ & $\mathrm{~N}$ & $\begin{array}{l}\text { Database username of person who most recently updated } \\
\text { record. }\end{array}$ \\
\hline
\end{tabular}


Basking Areas Table-Continued

\begin{tabular}{lcrcccl}
\hline Field Name & $\begin{array}{c}\text { Data } \\
\text { Type }\end{array}$ & $\begin{array}{c}\text { Field } \\
\text { Length }\end{array}$ & $\begin{array}{c}\text { Decimal } \\
\text { Places }\end{array}$ & $\begin{array}{c}\text { Required } \\
\text { Field }\end{array}$ & $\begin{array}{c}\text { Primary } \\
\text { Key }\end{array}$ & Definition \\
\hline RecordID & int & 0 & 0 & $\mathrm{~N}$ & $\mathrm{~N}$ & Pendragon PDA field, for PDA compatibility. \\
UnitID & int & 0 & 0 & $\mathrm{~N}$ & $\mathrm{~N}$ & Pendragon PDA field, for PDA compatibility. \\
UserName & varchar & 64 & 0 & $\mathrm{~N}$ & $\mathrm{~N}$ & Pendragon PDA field, for PDA compatibility. \\
\hline
\end{tabular}

\section{Behaviors Table}

\begin{tabular}{lcccccl}
\hline \multicolumn{1}{c}{ Field Name } & $\begin{array}{c}\text { Data } \\
\text { Type }\end{array}$ & $\begin{array}{c}\text { Field } \\
\text { Length }\end{array}$ & $\begin{array}{c}\text { Decimal } \\
\text { Places }\end{array}$ & $\begin{array}{c}\text { Required } \\
\text { Field }\end{array}$ & $\begin{array}{c}\text { Primary } \\
\text { Key }\end{array}$ & Definition \\
\hline Behavior & varchar & 50 & 0 & $\mathrm{~N}$ & $\mathrm{~N}$ & Behavior of observed taxon. See luBehavior. \\
BehaviorsID & varchar & 50 & 0 & $\mathrm{Y}$ & $\mathrm{Y}$ & Behaviors record identifier. \\
Created & datetime & 0 & 0 & $\mathrm{~N}$ & $\mathrm{~N}$ & Pendragon PDA field, for PDA compatibility. \\
CreatedBy & varchar & 64 & 0 & $\mathrm{Y}$ & $\mathrm{N}$ & Database username of person who created record. \\
CreatedDate & datetime & 0 & 0 & $\mathrm{Y}$ & $\mathrm{N}$ & Date and time when record was created. \\
DirectionOfBehavior & decimal & 4 & 1 & $\mathrm{~N}$ & $\mathrm{~N}$ & Decimal degree direction of behavior. \\
EditDate & datetime & 0 & 0 & $\mathrm{~N}$ & $\mathrm{~N}$ & Date and time when record was most recently updated. \\
EditedBy & varchar & 64 & 0 & $\mathrm{~N}$ & $\mathrm{~N}$ & Database username of person who most recently updated record. \\
QAQC_Note & varchar & 255 & 0 & $\mathrm{~N}$ & $\mathrm{~N}$ & Reason for change made to record during QAQC review. \\
QAQC_Status & varchar & 20 & 0 & $\mathrm{Y}$ & $\mathrm{N}$ & Describes current stage of QAQC. \\
RecordID & int & 0 & 0 & $\mathrm{~N}$ & $\mathrm{~N}$ & Pendragon PDA field, for PDA compatibility. \\
TaxaObservation & varchar & 50 & 0 & $\mathrm{~N}$ & $\mathrm{~N}$ & Associated TaxaObservationDetails record identifier. \\
$\quad$ & & & & & & \\
DetailsID & int & 0 & 0 & $\mathrm{~N}$ & $\mathrm{~N}$ & Pendragon PDA field, for PDA compatibility. \\
UnitID & varchar & 64 & 0 & $\mathrm{~N}$ & $\mathrm{~N}$ & Pendragon PDA field, for PDA compatibility. \\
\hline
\end{tabular}

\section{Capture Table}

\begin{tabular}{lcccccl}
\hline \multicolumn{1}{c}{ Field Name } & $\begin{array}{c}\text { Data } \\
\text { Type }\end{array}$ & $\begin{array}{c}\text { Field } \\
\text { Length }\end{array}$ & $\begin{array}{c}\text { Decimal } \\
\text { Places }\end{array}$ & $\begin{array}{c}\text { Required } \\
\text { Field }\end{array}$ & $\begin{array}{c}\text { Primary } \\
\text { Key }\end{array}$ & Definition \\
\hline AirwayClear & char & 3 & 0 & $\mathrm{~N}$ & $\mathrm{~N}$ & Was the airway clear for the capture. \\
CapID & varchar & 50 & 0 & $\mathrm{Y}$ & $\mathrm{Y}$ & Capture record identifier. \\
Created & datetime & 0 & 0 & $\mathrm{~N}$ & $\mathrm{~N}$ & Pendragon PDA field, for PDA compatibility. \\
CreatedBy & varchar & 64 & 0 & $\mathrm{Y}$ & $\mathrm{N}$ & Database username of person who created record. \\
CreatedDate & datetime & 0 & 0 & $\mathrm{Y}$ & $\mathrm{N}$ & Date and time when record was created. \\
EditDate & datetime & 0 & 0 & $\mathrm{~N}$ & $\mathrm{~N}$ & Date and time when record was most recently updated. \\
EditedBy & varchar & 64 & 0 & $\mathrm{~N}$ & $\mathrm{~N}$ & Database username of person who most recently updated record. \\
EyeOintment & char & 3 & 0 & $\mathrm{~N}$ & $\mathrm{~N}$ & Was eye ointment applied to capture. \\
IAID & varchar & 50 & 0 & $\mathrm{~N}$ & $\mathrm{~N}$ & Associated IndivAnimal record identifier. \\
RecordID & int & 0 & 0 & $\mathrm{~N}$ & $\mathrm{~N}$ & Pendragon PDA field, for PDA compatibility. \\
TimeAmbulatory & datetime & 0 & 0 & $\mathrm{~N}$ & $\mathrm{~N}$ & Time that capture was ambulatory or capable of movement. \\
TimeDown & datetime & 0 & 0 & $\mathrm{~N}$ & $\mathrm{~N}$ & Time that capture was immobilized. \\
TimeNotTractable & datetime & 0 & 0 & $\mathrm{~N}$ & $\mathrm{~N}$ & Time that capture was not under control. \\
UnitID & int & 0 & 0 & $\mathrm{~N}$ & $\mathrm{~N}$ & Pendragon PDA field, for PDA compatibility. \\
UserName & varchar & 64 & 0 & $\mathrm{~N}$ & $\mathrm{~N}$ & Pendragon PDA field, for PDA compatibility. \\
\hline
\end{tabular}




\section{ChemicalObservation Table}

\begin{tabular}{|c|c|c|c|c|c|c|}
\hline Field Name & $\begin{array}{l}\text { Data } \\
\text { Type }\end{array}$ & $\begin{array}{l}\text { Field } \\
\text { Length }\end{array}$ & $\begin{array}{c}\text { Decimal } \\
\text { Places }\end{array}$ & $\begin{array}{l}\text { Required } \\
\text { Field }\end{array}$ & $\begin{array}{l}\text { Primary } \\
\text { Key }\end{array}$ & Definition \\
\hline ANC & int & 0 & 0 & $\mathrm{~N}$ & $\mathrm{~N}$ & $\begin{array}{l}\text { Acid Neutralizing Capacity: ability of water to resist changes } \\
\text { in } \mathrm{pH} \text {. }\end{array}$ \\
\hline Chloride & decimal & 4 & 1 & $\mathrm{~N}$ & $\mathrm{~N}$ & Concentration of chloride in the water to nearest $0.001 \mathrm{mg} / \mathrm{L}$. \\
\hline Chlorophyll & decimal & 4 & 1 & $\mathrm{~N}$ & $\mathrm{~N}$ & Concentration of chlorophyll (mg/L). \\
\hline COID & varchar & 50 & 0 & $\mathrm{Y}$ & Y & ChemicalObservation record identifier. \\
\hline Created & datetime & 0 & 0 & $\mathrm{~N}$ & $\mathrm{~N}$ & Pendragon PDA field, for PDA compatibility. \\
\hline CreatedBy & varchar & 64 & 0 & $\mathrm{Y}$ & $\mathrm{N}$ & Database username of person who created record. \\
\hline CreatedDate & datetime & 0 & 0 & $\mathrm{Y}$ & $\mathrm{N}$ & Date and time when record was created. \\
\hline EditDate & datetime & 0 & 0 & $\mathrm{~N}$ & $\mathrm{~N}$ & Date and time when record was most recently updated. \\
\hline Nitrogen & decimal & 4 & 1 & $\mathrm{~N}$ & $\mathrm{~N}$ & Nitrogen concentration to nearest $0.1 \mathrm{mg} / \mathrm{L}$. \\
\hline Notes & varchar & 255 & 0 & $\mathrm{~N}$ & $\mathrm{~N}$ & Notes. \\
\hline ObservationDate & datetime & 0 & 0 & $\mathrm{~N}$ & $\mathrm{~N}$ & Date and time this water chemistry observation was made. \\
\hline ObservedByAffiliateID & int & 0 & 0 & $\mathrm{~N}$ & $\mathrm{~N}$ & ID of person making observation. See Affiliate table. \\
\hline $\mathrm{pH}$ & decimal & 4 & 1 & $\mathrm{~N}$ & $\mathrm{~N}$ & $\begin{array}{l}\text { An expression of the intensity of the basic or acid condition of } \\
\text { a liquid; may range from } 0 \text { to } 14 \text {, where } 0 \text { is the most acid } \\
\text { and } 7 \text { is neutral, measured to the nearest } 0.01 \text {. }\end{array}$ \\
\hline Phosphates & decimal & 5 & 3 & $\mathrm{~N}$ & $\mathrm{~N}$ & Concentration of phosphates in water $(\mathrm{mg} / \mathrm{L})$. \\
\hline PointID & varchar & 50 & 0 & $\mathrm{~N}$ & $\mathrm{~N}$ & Associated SpatialFeature record identifier. \\
\hline SurveyEventID & varchar & 50 & 0 & $\mathrm{Y}$ & $\mathrm{N}$ & Associated SurveyEvent record identifier. \\
\hline TotalAlk & decimal & 4 & 1 & $\mathrm{~N}$ & $\mathrm{~N}$ & Total alkalinity in water (ppm). \\
\hline UnitID & int & 0 & 0 & $\mathrm{~N}$ & $\mathrm{~N}$ & Pendragon PDA field, for PDA compatibility. \\
\hline UserName & varchar & 64 & 0 & $\mathrm{~N}$ & $\mathrm{~N}$ & Pendragon PDA field, for PDA compatibility. \\
\hline
\end{tabular}

\section{ChemicalTreatment Table}

\begin{tabular}{lcccccl}
\hline \multicolumn{1}{c}{ Field Name } & $\begin{array}{c}\text { Data } \\
\text { Type }\end{array}$ & $\begin{array}{c}\text { Field } \\
\text { Length }\end{array}$ & $\begin{array}{c}\text { Decimal } \\
\text { Places }\end{array}$ & $\begin{array}{c}\text { Required } \\
\text { Field }\end{array}$ & $\begin{array}{c}\text { Primary } \\
\text { Key }\end{array}$ & Definition \\
\hline AppEquipment & varchar & 25 & 0 & $\mathrm{~N}$ & $\mathrm{~N}$ & Equipment/vehicle used to apply the chemical. \\
AppliedBy & int & 0 & 0 & $\mathrm{~N}$ & $\mathrm{~N}$ & Affiliate who applied the treatment. \\
AppLocContext & varchar & 25 & 0 & $\mathrm{~N}$ & $\mathrm{~N}$ & General area within habitat that treatment occurred. \\
AppMethod & varchar & 25 & 0 & $\mathrm{~N}$ & $\mathrm{~N}$ & Method used to apply chemical. \\
AreaTreated & numeric & 8 & 4 & $\mathrm{~N}$ & $\mathrm{~N}$ & Acreage of the area treated. \\
ChemicalApplied & varchar & 25 & 0 & $\mathrm{~N}$ & $\mathrm{~N}$ & Name of herbicide/pesticide/insecticide.
\end{tabular}


ChemicalTreatment Table-Continued

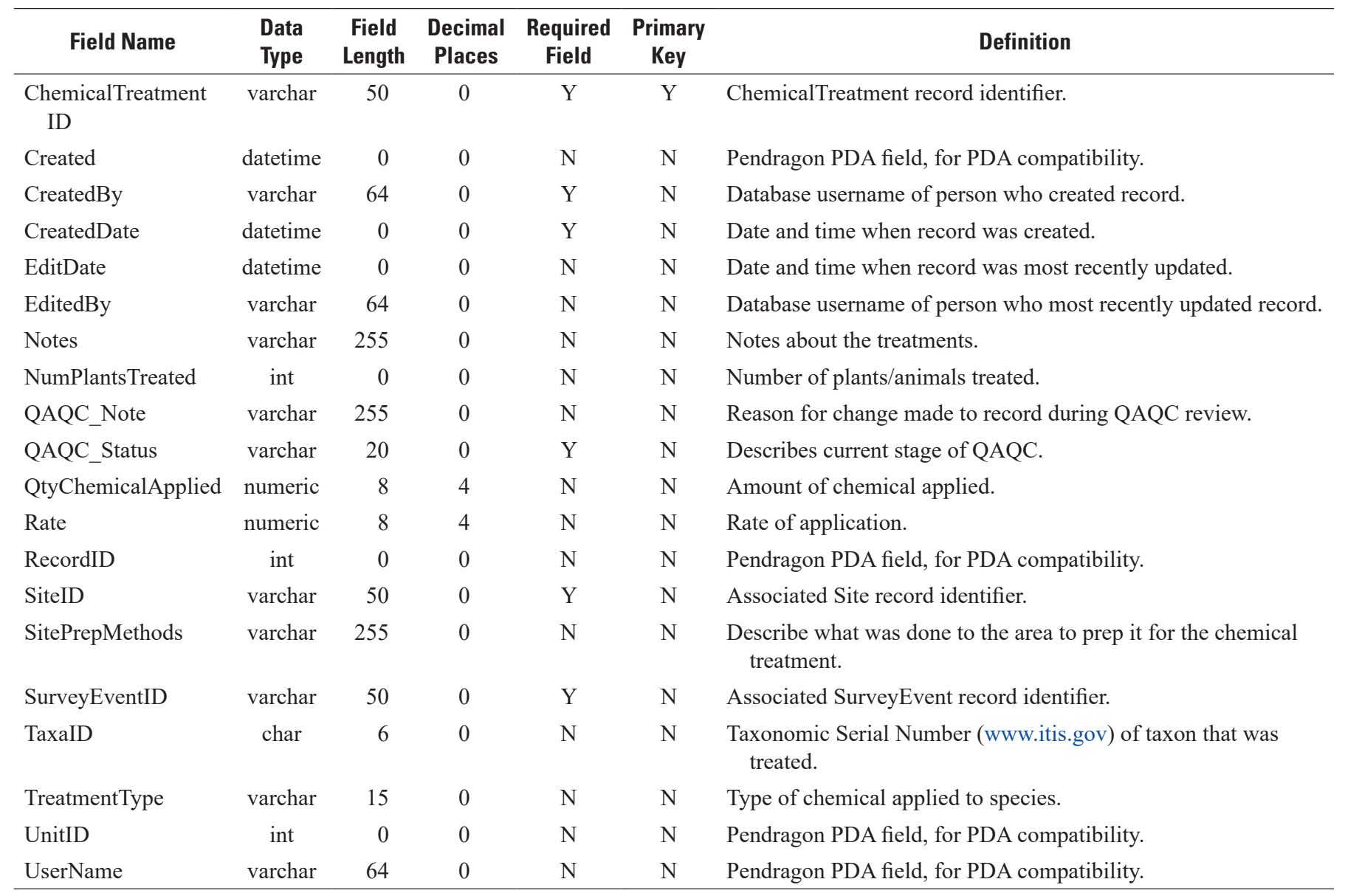

\section{Citation Table}

\begin{tabular}{|c|c|c|c|c|c|c|}
\hline Field Name & Data Type & $\begin{array}{c}\text { Field } \\
\text { Length }\end{array}$ & $\begin{array}{l}\text { Decimal } \\
\text { Places }\end{array}$ & $\begin{array}{l}\text { Required } \\
\text { Field }\end{array}$ & $\begin{array}{c}\text { Primary } \\
\text { Key }\end{array}$ & Definition \\
\hline ArticleDate & Smalldatetime & 0 & 0 & $\mathrm{~N}$ & $\mathrm{~N}$ & Date of publication. \\
\hline ArticleType & varchar & 50 & 0 & $\mathrm{Y}$ & $\mathrm{N}$ & Type of publication (tech report, journal, and so forth). \\
\hline ArtNotes & varchar & 200 & 0 & $\mathrm{~N}$ & $\mathrm{~N}$ & Notes in reference to article. \\
\hline AuthorID3 & varchar & 50 & 0 & $\mathrm{~N}$ & $\mathrm{~N}$ & Tertiary author. \\
\hline AuthorID4 & varchar & 50 & 0 & $\mathrm{~N}$ & $\mathrm{~N}$ & Quaternary author. \\
\hline AuthorID5 & varchar & 50 & 0 & $\mathrm{~N}$ & $\mathrm{~N}$ & Pentanary author. \\
\hline CitationID & varchar & 50 & 0 & $\mathrm{Y}$ & Y & Citation record identifier. \\
\hline EditedBy & varchar & 64 & 0 & $\mathrm{~N}$ & $\mathrm{~N}$ & Database username of person who most recently updated record. \\
\hline EFIID & bigint & 19 & 0 & $\mathrm{~N}$ & $\mathrm{~N}$ & Associated ExternalFileInfo record identifier. \\
\hline PubCity & varchar & 50 & 0 & $\mathrm{~N}$ & $\mathrm{~N}$ & City of publication. \\
\hline PubCountry & varchar & 50 & 0 & $\mathrm{~N}$ & $\mathrm{~N}$ & Country of publication. \\
\hline
\end{tabular}


Citation Table-Continued

\begin{tabular}{lccccccl}
\hline Field Name & Data Type & $\begin{array}{c}\text { Field } \\
\text { Length }\end{array}$ & $\begin{array}{c}\text { Decimal } \\
\text { Places }\end{array}$ & $\begin{array}{c}\text { Required } \\
\text { Field }\end{array}$ & $\begin{array}{c}\text { Primary } \\
\text { Key }\end{array}$ & Definition \\
\hline PublisherName & varchar & 100 & 0 & $\mathrm{~N}$ & $\mathrm{~N}$ & Name of publisher. \\
TechName & varchar & 200 & 0 & $\mathrm{~N}$ & $\mathrm{~N}$ & Name of office producing technical report. \\
\hline
\end{tabular}

Citation_Collection_Affiliate Table

\begin{tabular}{lcccccl}
\hline \multicolumn{1}{c}{ Field Name } & $\begin{array}{c}\text { Data } \\
\text { Type }\end{array}$ & $\begin{array}{c}\text { Field } \\
\text { Length }\end{array}$ & $\begin{array}{c}\text { Decimal } \\
\text { Places }\end{array}$ & $\begin{array}{c}\text { Required } \\
\text { Field }\end{array}$ & $\begin{array}{c}\text { Primary } \\
\text { Key }\end{array}$ & Definition \\
\hline CitationID & varchar & 50 & 0 & $\mathrm{Y}$ & $\mathrm{Y}$ & Citation record identifier. \\
$\begin{array}{l}\text { CollectionAffil- } \\
\quad \text { iateID }\end{array}$ & int & 0 & 0 & $\mathrm{Y}$ & $\mathrm{Y}$ & Collection_Affiliate record identifier. \\
CreatedBy & varchar & 64 & 0 & $\mathrm{Y}$ & $\mathrm{N}$ & Database username of person who created record. \\
CreatedDate & datetime & 0 & 0 & $\mathrm{Y}$ & $\mathrm{N}$ & Date and time when record was created. \\
EditDate & datetime & 0 & 0 & $\mathrm{~N}$ & $\mathrm{~N}$ & Date and time when record was most recently updated. \\
EditedBy & varchar & 64 & 0 & $\mathrm{~N}$ & $\mathrm{~N}$ & Database username of person who most recently updated record. \\
\hline
\end{tabular}

\section{Collection Table}

\begin{tabular}{|c|c|c|c|c|c|c|}
\hline Field Name & $\begin{array}{l}\text { Data } \\
\text { Type }\end{array}$ & $\begin{array}{l}\text { Field } \\
\text { Length }\end{array}$ & $\begin{array}{l}\text { Decimal } \\
\text { Places }\end{array}$ & $\begin{array}{l}\text { Required } \\
\text { Field }\end{array}$ & $\begin{array}{l}\text { Primary } \\
\text { Key }\end{array}$ & Definition \\
\hline AmtWaterFiltered & decimal & 7 & 2 & $\mathrm{~N}$ & $\mathrm{~N}$ & $\begin{array}{l}\text { For filter collection types, records how many milliliters of } \\
\text { water were pumped through the filter. }\end{array}$ \\
\hline BoxName & varchar & 10 & 0 & $\mathrm{~N}$ & $\mathrm{~N}$ & Box ID within freezer. \\
\hline CollectionID & varchar & 50 & 0 & $\mathrm{Y}$ & $\mathrm{Y}$ & Collection record identifier. \\
\hline CollectionType & varchar & 10 & 0 & $\mathrm{~N}$ & $\mathrm{~N}$ & $\begin{array}{l}\text { Description of physical type of collection. See } \\
\text { luCollectionType. }\end{array}$ \\
\hline ColumnName & varchar & 10 & 0 & $\mathrm{~N}$ & $\mathrm{~N}$ & Column ID within freezer. \\
\hline CompactingShelf & varchar & 20 & 0 & $\mathrm{~N}$ & $\mathrm{~N}$ & $\begin{array}{l}\text { Name of San Diego Field Station compacting shelf where } \\
\text { collection is stored. }\end{array}$ \\
\hline ConvertedToDNA & bit & 0 & 0 & $\mathrm{~N}$ & $\mathrm{~N}$ & If true, tissue was converted to DNA. \\
\hline Created & datetime & 0 & 0 & $\mathrm{~N}$ & $\mathrm{~N}$ & Pendragon PDA field, for PDA compatibility. \\
\hline CurrentStorageMedium & varchar & 30 & 0 & $\mathrm{~N}$ & $\mathrm{~N}$ & $\begin{array}{l}\text { Description of container currently used to store collected } \\
\text { item. }\end{array}$ \\
\hline Depleted & bit & 0 & 0 & $\mathrm{~N}$ & $\mathrm{~N}$ & If true, entirety of collection has been used; nothing left. \\
\hline DNAConcentration & decimal & 5 & 1 & $\mathrm{~N}$ & $\mathrm{~N}$ & $\begin{array}{l}\text { Concentration of DNA used in extraction. Measured in } \\
\text { nanograms per microliter or (ng/ul). }\end{array}$ \\
\hline EditDate & datetime & 0 & 0 & $\mathrm{~N}$ & $\mathrm{~N}$ & Date and time when record was most recently updated. \\
\hline EditedBy & varchar & 64 & 0 & $\mathrm{~N}$ & $\mathrm{~N}$ & $\begin{array}{l}\text { Database username of person who most recently updated } \\
\text { record. }\end{array}$ \\
\hline
\end{tabular}


Collection Table-Continued

\begin{tabular}{|c|c|c|c|c|c|c|}
\hline Field Name & $\begin{array}{l}\text { Data } \\
\text { Type }\end{array}$ & $\begin{array}{l}\text { Field } \\
\text { Length }\end{array}$ & $\begin{array}{l}\text { Decimal } \\
\text { Places }\end{array}$ & $\begin{array}{l}\text { Required } \\
\text { Field }\end{array}$ & $\begin{array}{l}\text { Primary } \\
\text { Key }\end{array}$ & Definition \\
\hline FromCollectionID & varchar & 50 & 0 & $\mathrm{~N}$ & $\mathrm{~N}$ & $\begin{array}{l}\text { CollectionID of collection from which this portion of } \\
\text { collection was taken. }\end{array}$ \\
\hline GeneratedID & varchar & 50 & 0 & $\mathrm{~N}$ & $\mathrm{~N}$ & $\begin{array}{l}\text { Unique alpha-numeric value generated at the site of } \\
\text { collection, used to identify this collection of tissue, } \\
\text { voucher, swab, or other. This value is used to mark the } \\
\text { vial or other container used to store the collection and is } \\
\text { retained through the lifetime of the specimen and data } \\
\text { record. }\end{array}$ \\
\hline IAID & varchar & 50 & 0 & $\mathrm{~N}$ & $\mathrm{~N}$ & Associated IndivAnimals record identifier. \\
\hline Notes & varchar & 250 & 0 & $\mathrm{~N}$ & $\mathrm{~N}$ & Notes about the Collection record. \\
\hline OriginalStorageMedium & varchar & 30 & 0 & $\mathrm{~N}$ & $\mathrm{~N}$ & $\begin{array}{l}\text { Description of container originally used to store collected } \\
\text { item. }\end{array}$ \\
\hline QAQC_Note & varchar & 255 & 0 & $\mathrm{~N}$ & $\mathrm{~N}$ & Reason for change made to record during QAQC review. \\
\hline QAQC_Status & varchar & 20 & 0 & $\mathrm{Y}$ & $\mathrm{N}$ & Describes current stage of QAQC. \\
\hline Rack & varchar & 10 & 0 & $\mathrm{~N}$ & $\mathrm{~N}$ & Rack ID within freezer. \\
\hline RecordID & int & 0 & 0 & $\mathrm{~N}$ & $\mathrm{~N}$ & Pendragon PDA field, for PDA compatibility. \\
\hline RowName & varchar & 10 & 0 & $\mathrm{~N}$ & $\mathrm{~N}$ & Row ID within freezer. \\
\hline TissueTypeID & char & 2 & 0 & $\mathrm{~N}$ & $\mathrm{~N}$ & TissueType record identifier. See TissType. \\
\hline UnitID & int & 0 & 0 & $\mathrm{~N}$ & $\mathrm{~N}$ & Pendragon PDA field, for PDA compatibility. \\
\hline UserName & varchar & 64 & 0 & $\mathrm{~N}$ & $\mathrm{~N}$ & Pendragon PDA field, for PDA compatibility. \\
\hline WPOID & varchar & 50 & 0 & $\mathrm{~N}$ & $\mathrm{~N}$ & Associated Water PhysicalObservation record identifier. \\
\hline
\end{tabular}

\section{Collection_Affilliate Table}

\begin{tabular}{|c|c|c|c|c|c|c|}
\hline Field Name & $\begin{array}{l}\text { Data } \\
\text { Type }\end{array}$ & $\begin{array}{l}\text { Field } \\
\text { Length }\end{array}$ & $\begin{array}{l}\text { Decimal } \\
\text { Places }\end{array}$ & $\begin{array}{l}\text { Required } \\
\text { Field }\end{array}$ & $\begin{array}{l}\text { Primary } \\
\text { Key }\end{array}$ & Definition \\
\hline AffiliateID & int & 0 & 0 & $\mathrm{Y}$ & $\mathrm{N}$ & Associated Affiliate record identifier. \\
\hline CollectionID & varchar & 50 & 0 & Y & $\mathrm{N}$ & Associated Collection record identifier. \\
\hline CollectionUse & varchar & 25 & 0 & $\mathrm{~N}$ & $\mathrm{~N}$ & $\begin{array}{l}\text { Use of the collected sample for the affiliate who requested it. } \\
\text { See luCollectionUses. }\end{array}$ \\
\hline CreatedBy & varchar & 64 & 0 & Y & $\mathrm{N}$ & Database username of person who created record. \\
\hline CreatedDate & datetime & 0 & 0 & Y & $\mathrm{N}$ & Date and time when record was created. \\
\hline CustodianRefID & varchar & 30 & 0 & $\mathrm{~N}$ & $\mathrm{~N}$ & $\begin{array}{l}\text { The reference ID used by the entity that is or was in possession } \\
\text { of collection item. }\end{array}$ \\
\hline DateEnd & datetime & 0 & 0 & $\mathrm{~N}$ & $\mathrm{~N}$ & Date item expected to return, if return is expected. \\
\hline EditDate & datetime & 0 & 0 & $\mathrm{~N}$ & $\mathrm{~N}$ & Date and time when record was most recently updated. \\
\hline EditedBy & varchar & 64 & 0 & $\mathrm{~N}$ & $\mathrm{~N}$ & Database username of person who most recently updated record. \\
\hline Notes & varchar & 250 & 0 & $\mathrm{~N}$ & $\mathrm{~N}$ & Notes about the Collection_Affiliate record. \\
\hline RecordID & int & 0 & 0 & $\mathrm{~N}$ & $\mathrm{~N}$ & Pendragon PDA field, for PDA compatibility. \\
\hline
\end{tabular}


Collection_Affilliate Table-Continued

\begin{tabular}{lcccccc}
\hline Field Name & $\begin{array}{c}\text { Data } \\
\text { Type }\end{array}$ & $\begin{array}{c}\text { Field } \\
\text { Length }\end{array}$ & $\begin{array}{c}\text { Decimal } \\
\text { Places }\end{array}$ & $\begin{array}{c}\text { Required } \\
\text { Field }\end{array}$ & $\begin{array}{c}\text { Primary } \\
\text { Key }\end{array}$ & Definition \\
\hline ReturnExpected & varchar & 10 & 0 & $\mathrm{~N}$ & $\mathrm{~N}$ & $\begin{array}{c}\text { If true, item is expected to be returned by DateEnd. See } \\
\text { luReturnExpected. }\end{array}$ \\
UnitID & int & 0 & 0 & $\mathrm{~N}$ & $\mathrm{~N}$ & $\begin{array}{c}\text { Pendragon PDA field, for PDA compatibility. } \\
\text { UserName }\end{array}$ \\
\hline
\end{tabular}

\section{Communication Table}

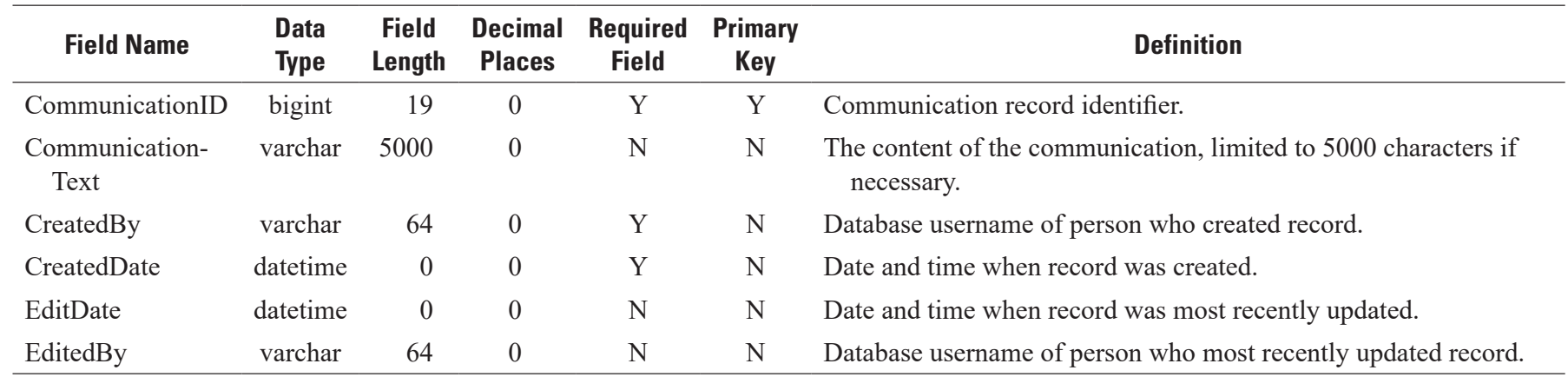

\section{DistanceTo Table}

\begin{tabular}{|c|c|c|c|c|c|c|}
\hline Field Name & $\begin{array}{l}\text { Data } \\
\text { Type }\end{array}$ & $\begin{array}{c}\text { Field } \\
\text { Length }\end{array}$ & $\begin{array}{l}\text { Decimal } \\
\text { Places }\end{array}$ & $\begin{array}{l}\text { Required } \\
\text { Field }\end{array}$ & $\begin{array}{l}\text { Primary } \\
\text { Key }\end{array}$ & Definition \\
\hline Created & datetime & 0 & 0 & $\mathrm{~N}$ & $\mathrm{~N}$ & Pendragon PDA field, for PDA compatibility. \\
\hline CreatedDate & datetime & 0 & 0 & $\mathrm{Y}$ & $\mathrm{N}$ & Date and time when record was created. \\
\hline Direction & numeric & 3 & 0 & $\mathrm{~N}$ & $\mathrm{~N}$ & Measured in compass degrees. \\
\hline DTID & varchar & 50 & 0 & Y & Y & DistanceTo record identifier. \\
\hline EditDate & datetime & 0 & 0 & $\mathrm{~N}$ & $\mathrm{~N}$ & Date and time when record was most recently updated. \\
\hline DistEstMethod & varchar & 20 & 0 & $\mathrm{~N}$ & $\mathrm{~N}$ & $\begin{array}{l}\text { How the distance was measured. Methods include actual measurement } \\
\text { while in the field, estimated (ball-parked), or already known from a } \\
\text { map or similar. See luEstMethod. }\end{array}$ \\
\hline EditDate & datetime & 0 & 0 & $\mathrm{~N}$ & $\mathrm{~N}$ & Date and time when record was most recently updated. \\
\hline EditedBy & varchar & 64 & 0 & $\mathrm{~N}$ & $\mathrm{~N}$ & Database username of person who most recently updated record. \\
\hline Notes & varchar & 250 & 0 & $\mathrm{~N}$ & $\mathrm{~N}$ & Notes about the DistanceTo record. \\
\hline OtherItem & varchar & 75 & 0 & $\mathrm{~N}$ & $\mathrm{~N}$ & $\begin{array}{l}\text { Description of feature/object/item to which direction and distance } \\
\text { is being recorded. Optional, as measurement could be to a } \\
\text { TaxaObservation record instead. }\end{array}$ \\
\hline PODID & varchar & 50 & 0 & $\mathrm{~N}$ & $\mathrm{~N}$ & $\begin{array}{l}\text { Optional foreign key, as this record may relate to a } \\
\text { PlantObservationDetails instead. }\end{array}$ \\
\hline QAQC_Note & varchar & 255 & 0 & $\mathrm{~N}$ & $\mathrm{~N}$ & Reason for change made to record during QAQC review. \\
\hline
\end{tabular}




\begin{tabular}{|c|c|c|c|c|c|c|}
\hline Field Name & $\begin{array}{l}\text { Data } \\
\text { Type }\end{array}$ & $\begin{array}{c}\text { Field } \\
\text { Length }\end{array}$ & $\begin{array}{l}\text { Decimal } \\
\text { Places }\end{array}$ & $\begin{array}{l}\text { Required } \\
\text { Field }\end{array}$ & $\begin{array}{c}\text { Primary } \\
\text { Key }\end{array}$ & Definition \\
\hline Taxa & varchar & 50 & 0 & $\mathrm{~N}$ & $\mathrm{~N}$ & $\begin{array}{l}\text { Reference to TaxaObservation record to which direction and distance } \\
\text { is being recorded. Optional, as measurement could be to Other Item } \\
\text { instead. }\end{array}$ \\
\hline TODID & varchar & 50 & 0 & $\mathrm{~N}$ & $\mathrm{~N}$ & $\begin{array}{l}\text { Optional foreign key, as this record may relate to a } \\
\text { TaxaObservationDetails record instead. }\end{array}$ \\
\hline UnitID & int & 0 & 0 & $\mathrm{~N}$ & $\mathrm{~N}$ & Pendragon PDA field, for PDA compatibility. \\
\hline UserName & varchar & 64 & 0 & $\mathrm{~N}$ & $\mathrm{~N}$ & Pendragon PDA field, for PDA compatibility. \\
\hline
\end{tabular}

\section{Disturbance Table}

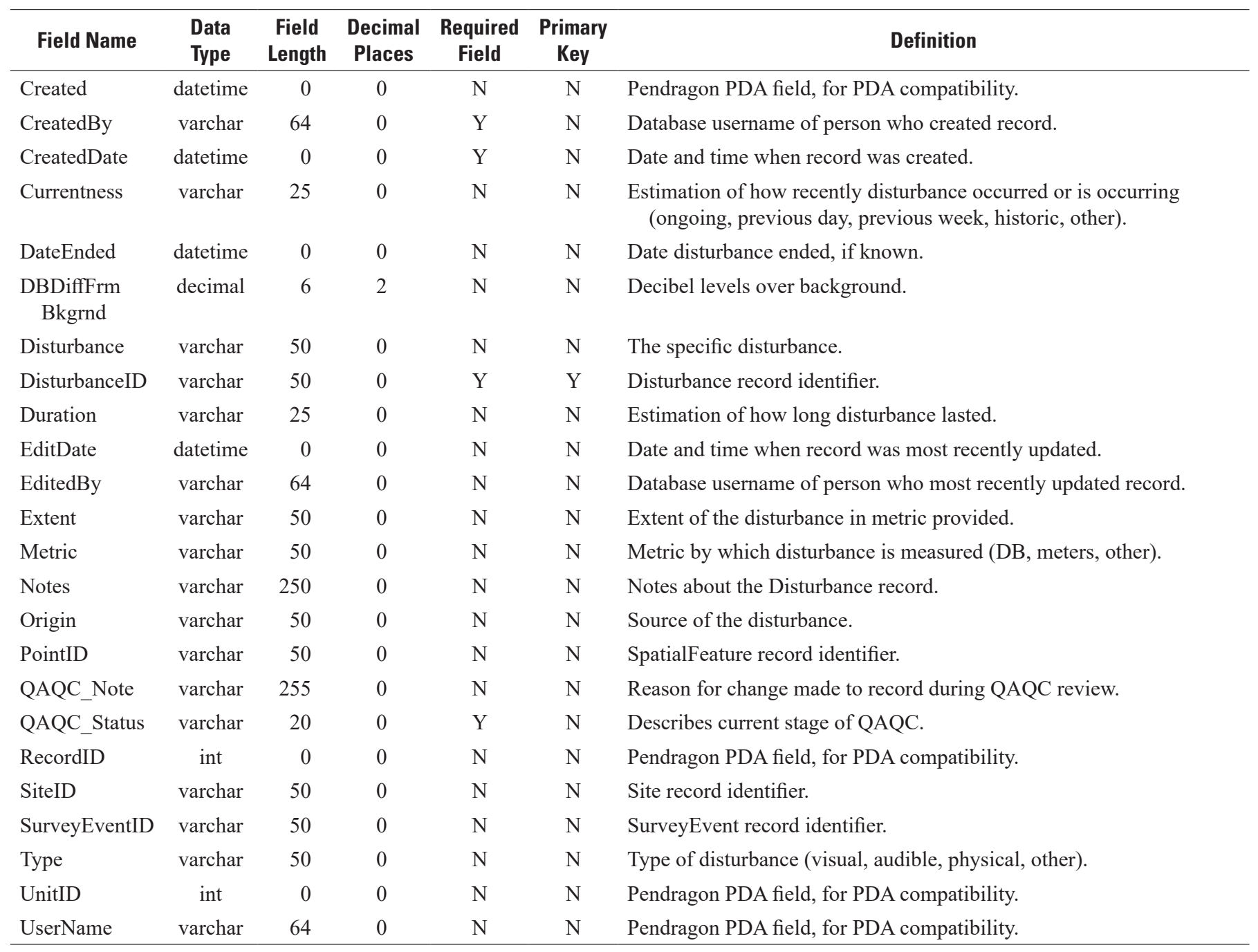


EggmassCounts Table

\begin{tabular}{lcccccl}
\hline Field Name & $\begin{array}{c}\text { Data } \\
\text { Type }\end{array}$ & $\begin{array}{c}\text { Field } \\
\text { Length }\end{array}$ & $\begin{array}{c}\text { Decimal } \\
\text { Places }\end{array}$ & $\begin{array}{c}\text { Required } \\
\text { Field }\end{array}$ & $\begin{array}{c}\text { Primary } \\
\text { Key }\end{array}$ & Definition \\
\hline Count & int & 0 & 0 & $\mathrm{Y}$ & $\mathrm{N}$ & Count of egg masses. \\
CreatedBy & varchar & 64 & 0 & $\mathrm{Y}$ & $\mathrm{N}$ & Database username of person who created record. \\
CreatedDate & datetime & 0 & 0 & $\mathrm{Y}$ & $\mathrm{N}$ & Date and time when record was created. \\
ECID & bigint & 19 & 0 & $\mathrm{Y}$ & $\mathrm{Y}$ & EggmassCounts record identifier. \\
EditDate & datetime & 0 & 0 & $\mathrm{~N}$ & $\mathrm{~N}$ & Date and time when record was most recently updated. \\
EditedBy & varchar & 64 & 0 & $\mathrm{~N}$ & $\mathrm{~N}$ & Database username of person who most recently updated record. \\
ESID & varchar & 50 & 0 & $\mathrm{Y}$ & $\mathrm{N}$ & EstimateSeries record identifier. \\
Notes & varchar & 250 & 0 & $\mathrm{~N}$ & $\mathrm{~N}$ & Notes about the eggmass count. \\
QAQC_Note & varchar & 255 & 0 & $\mathrm{~N}$ & $\mathrm{~N}$ & Reason for change made to record during QAQC review. \\
QAQC_Status & varchar & 20 & 0 & $\mathrm{Y}$ & $\mathrm{N}$ & Describes current stage of QAQC. \\
Season & varchar & 15 & 0 & $\mathrm{~N}$ & $\mathrm{~N}$ & The season of the year to which the estimates apply. \\
Year & varchar & 25 & 0 & $\mathrm{~N}$ & $\mathrm{~N}$ & The year to which this count applies. \\
\hline
\end{tabular}

\section{ElectronicFileInfo Table}

\begin{tabular}{|c|c|c|c|c|c|c|}
\hline Field Name & $\begin{array}{l}\text { Data } \\
\text { Type }\end{array}$ & $\begin{array}{l}\text { Field } \\
\text { Length }\end{array}$ & $\begin{array}{l}\text { Decimal } \\
\text { Places }\end{array}$ & $\begin{array}{l}\text { Required } \\
\text { Field }\end{array}$ & $\begin{array}{l}\text { Primary } \\
\text { Key }\end{array}$ & Definition \\
\hline AccessLevel & char & 10 & 0 & $\mathrm{~N}$ & $\mathrm{~N}$ & $\begin{array}{l}\text { Code used to specify what level of access is required to obtain this } \\
\text { file; the specifics of this method not presently implemented. }\end{array}$ \\
\hline CreatedBy & varchar & 64 & 0 & $\mathrm{Y}$ & $\mathrm{N}$ & Database username of person who created record. \\
\hline CreatedDate & datetime & 0 & 0 & $\mathrm{Y}$ & $\mathrm{N}$ & Date and time when record was created. \\
\hline EditDate & datetime & 0 & 0 & $\mathrm{~N}$ & $\mathrm{~N}$ & Date and time when record was most recently updated. \\
\hline EFIID & bigint & 19 & 0 & Y & $\mathrm{Y}$ & ElectronicFileInfo record identifier. \\
\hline FileDescription & varchar & 200 & 0 & $\mathrm{~N}$ & $\mathrm{~N}$ & Description of the file, provided by the user. \\
\hline FileOwner & int & 0 & 0 & $\mathrm{~N}$ & $\mathrm{~N}$ & $\begin{array}{l}\text { Associated Affiliate record identifier to identify the owner of the } \\
\text { file. }\end{array}$ \\
\hline FRID & bigint & 19 & 0 & $\mathrm{~N}$ & $\mathrm{~N}$ & Associated FileRepository record identifier. \\
\hline OriginalFileName & varchar & 100 & 0 & Y & $\mathrm{N}$ & $\begin{array}{l}\text { The name of the file when it was added to the system, as provided } \\
\text { by the user. }\end{array}$ \\
\hline PhotoID & varchar & 50 & 0 & $\mathrm{~N}$ & $\mathrm{~N}$ & $\begin{array}{l}\text { Associated PhotoInfo record identifier, if this record is related to a } \\
\text { PhotoInfo record. }\end{array}$ \\
\hline URL & varchar & 250 & 0 & $\mathrm{~N}$ & $\mathrm{~N}$ & The web address of the file, if applicable. \\
\hline
\end{tabular}


ElectronicFilelnfo_Keywords Table

\begin{tabular}{lcccccc}
\hline Field Name & $\begin{array}{c}\text { Data } \\
\text { Type }\end{array}$ & $\begin{array}{c}\text { Field } \\
\text { Length }\end{array}$ & $\begin{array}{c}\text { Decimal } \\
\text { Places }\end{array}$ & $\begin{array}{c}\text { Required } \\
\text { Field }\end{array}$ & $\begin{array}{c}\text { Primary } \\
\text { Key }\end{array}$ & Definition \\
\hline CreatedBy & varchar & 64 & 0 & $\mathrm{Y}$ & $\mathrm{N}$ & Database username of person who created record. \\
CreatedDate & datetime & 0 & 0 & $\mathrm{Y}$ & $\mathrm{N}$ & Date and time when record was created. \\
EditDate & datetime & 0 & 0 & $\mathrm{~N}$ & $\mathrm{~N}$ & Date and time when record was most recently updated. \\
EditedBy & varchar & 64 & 0 & $\mathrm{~N}$ & $\mathrm{~N}$ & Database username of person who most recently updated record. \\
EFIID & bigint & 19 & 0 & $\mathrm{Y}$ & $\mathrm{Y}$ & Associated ElectronicFileInfo record identifier. \\
KeywordID & bigint & 19 & 0 & $\mathrm{Y}$ & $\mathrm{Y}$ & Associated Keyword record identifier. \\
\hline
\end{tabular}

\section{EstimateSeries Table}

\begin{tabular}{|c|c|c|c|c|c|c|}
\hline Field Name & $\begin{array}{l}\text { Data } \\
\text { Type }\end{array}$ & $\begin{array}{l}\text { Field } \\
\text { Length }\end{array}$ & $\begin{array}{l}\text { Decimal } \\
\text { Places }\end{array}$ & $\begin{array}{l}\text { Required } \\
\text { Field }\end{array}$ & $\begin{array}{l}\text { Primary } \\
\text { Key }\end{array}$ & Definition \\
\hline CreatedBy & varchar & 64 & 0 & $\mathrm{Y}$ & $\mathrm{N}$ & Database username of person who created record. \\
\hline EditDate & datetime & 0 & 0 & $\mathrm{~N}$ & $\mathrm{~N}$ & Date and time when record was most recently updated. \\
\hline EditedBy & varchar & 64 & 0 & $\mathrm{~N}$ & $\mathrm{~N}$ & Database username of person who most recently updated record. \\
\hline Model & varchar & 255 & 0 & $\mathrm{~N}$ & $\mathrm{~N}$ & $\begin{array}{l}\text { Notes regarding how occupancy was modeled in this observation/ } \\
\text { case. }\end{array}$ \\
\hline Notes & varchar & 250 & 0 & $\mathrm{~N}$ & $\mathrm{~N}$ & Notes about the estimate series record. \\
\hline $\begin{array}{l}\text { NumberOfSites } \\
\text { Sampled }\end{array}$ & int & 0 & 0 & $\mathrm{~N}$ & $\mathrm{~N}$ & Number of sites sampled in inference area. \\
\hline PEID & varchar & 50 & 0 & $\mathrm{Y}$ & $\mathrm{N}$ & Associated PopulationEstimates record identifier. \\
\hline QAQC_Note & varchar & 255 & 0 & $\mathrm{~N}$ & $\mathrm{~N}$ & Reason for change made to record during QAQC review. \\
\hline QAQC_Status & varchar & 20 & 0 & $\mathrm{Y}$ & $\mathrm{N}$ & Describes current stage of QAQC. \\
\hline Sex & int & 0 & 0 & $\mathrm{~N}$ & $\mathrm{~N}$ & Sex of species observed. See luGender. \\
\hline TaxaID & varchar & 25 & 0 & $\mathrm{Y}$ & $\mathrm{N}$ & $\begin{array}{l}\text { Taxonomic Serial Number (www.itis.gov) of species estimate is } \\
\text { applied to. }\end{array}$ \\
\hline Use & bit & 0 & 0 & $\mathrm{Y}$ & $\mathrm{N}$ & $\begin{array}{l}\text { Indicates whether or not this time series should be included in a } \\
\text { national synthesis. }\end{array}$ \\
\hline
\end{tabular}

ExternalReleaseTracking Table

\begin{tabular}{lcccccl}
\hline \multicolumn{1}{c}{ Field Name } & $\begin{array}{c}\text { Data } \\
\text { Type }\end{array}$ & $\begin{array}{c}\text { Field } \\
\text { Length }\end{array}$ & $\begin{array}{c}\text { Decimal } \\
\text { Places }\end{array}$ & $\begin{array}{c}\text { Required } \\
\text { Field }\end{array}$ & $\begin{array}{c}\text { Primary } \\
\text { Key }\end{array}$ & Definition \\
\hline ApprovalBy & int & 0 & 0 & $\mathrm{~N}$ & $\mathrm{~N}$ & Affiliate who approved the release of the dataset. \\
Comments & varchar & 250 & 0 & $\mathrm{~N}$ & $\mathrm{~N}$ & Comments regarding the dataset that was released. \\
CreatedBy & varchar & 64 & 0 & $\mathrm{Y}$ & $\mathrm{N}$ & Database username of person who created record. \\
CreatedDate & datetime & 0 & 0 & $\mathrm{Y}$ & $\mathrm{N}$ & Date and time when record was created. \\
DATE & datetime & 0 & 0 & $\mathrm{~N}$ & $\mathrm{~N}$ & Date the dataset was submitted to external source. \\
EditDate & datetime & 0 & 0 & $\mathrm{~N}$ & $\mathrm{~N}$ & Date and time when record was most recently updated. \\
EditedBy & varchar & 64 & 0 & $\mathrm{~N}$ & $\mathrm{~N}$ & Database username of person who most recently updated record. \\
ExternalReleaseID & varchar & 50 & 0 & $\mathrm{Y}$ & $\mathrm{Y}$ & ExternalReleaseTracking record identifier.
\end{tabular}


ExternalReleaseTracking Table-Continued

\begin{tabular}{|c|c|c|c|c|c|c|}
\hline Field Name & $\begin{array}{l}\text { Data } \\
\text { Type }\end{array}$ & $\begin{array}{l}\text { Field } \\
\text { Length }\end{array}$ & $\begin{array}{l}\text { Decimal } \\
\text { Places }\end{array}$ & $\begin{array}{l}\text { Required } \\
\text { Field }\end{array}$ & $\begin{array}{l}\text { Primary } \\
\text { Key }\end{array}$ & Definition \\
\hline ExternalSite & varchar & 50 & 0 & $\mathrm{~N}$ & $\mathrm{~N}$ & Site that the dataset was submitted to. \\
\hline ReleaseNotice & varchar & 100 & 0 & $\mathrm{~N}$ & $\mathrm{~N}$ & Link to Intent of Dataset Use PDF document. \\
\hline SurveyDescriptionID & int & 0 & 0 & $\mathrm{~N}$ & $\mathrm{~N}$ & $\begin{array}{l}\text { SurveyDescription record identifier of study that dataset is part } \\
\text { of. }\end{array}$ \\
\hline
\end{tabular}

FileRepositories Table

\begin{tabular}{lcccccl}
\hline \multicolumn{1}{c}{ Field Name } & $\begin{array}{c}\text { Data } \\
\text { Type }\end{array}$ & $\begin{array}{c}\text { Field } \\
\text { Length }\end{array}$ & $\begin{array}{c}\text { Decimal } \\
\text { Places }\end{array}$ & $\begin{array}{c}\text { Required } \\
\text { Field }\end{array}$ & $\begin{array}{c}\text { Primary } \\
\text { Key }\end{array}$ & Definition \\
\hline CreatedBy & varchar & 64 & 0 & $\mathrm{Y}$ & $\mathrm{N}$ & Database username of person who created record. \\
CreatedDate & datetime & 0 & 0 & $\mathrm{Y}$ & $\mathrm{N}$ & Date and time when record was created. \\
EditDate & datetime & 0 & 0 & $\mathrm{~N}$ & $\mathrm{~N}$ & Date and time when record was most recently updated. \\
EditedBy & varchar & 64 & 0 & $\mathrm{~N}$ & $\mathrm{~N}$ & Database username of person who most recently updated record. \\
FRID & bigint & 19 & 0 & $\mathrm{Y}$ & $\mathrm{Y}$ & FileRepositories record identifier. \\
OurLoginName & varchar & 100 & 0 & $\mathrm{~N}$ & $\mathrm{~N}$ & Username used to access repository. \\
OurPassword & varchar & 50 & 0 & $\mathrm{~N}$ & $\mathrm{~N}$ & Password used to access repository. \\
RepositoryName & int & 0 & 0 & $\mathrm{Y}$ & $\mathrm{N}$ & Affiliate record identifier identifying the location where the file is \\
& & & & & & managed. \\
RepositoryPOC & int & 0 & 0 & $\mathrm{Y}$ & $\mathrm{N}$ & Affiliate record identifier of the point of contact at the repository. \\
\hline
\end{tabular}

FocalSpeciesList Table

\begin{tabular}{|c|c|c|c|c|c|c|}
\hline Field Name & $\begin{array}{l}\text { Data } \\
\text { Type }\end{array}$ & $\begin{array}{l}\text { Field } \\
\text { Length }\end{array}$ & $\begin{array}{c}\text { Decimal } \\
\text { Places }\end{array}$ & $\begin{array}{l}\text { Required } \\
\text { Field }\end{array}$ & $\begin{array}{l}\text { Primary } \\
\text { Key }\end{array}$ & Definition \\
\hline CreatedBy & varchar & 64 & 0 & $\mathrm{Y}$ & $\mathrm{N}$ & Database username of person who created record. \\
\hline EditDate & datetime & 0 & 0 & $\mathrm{~N}$ & $\mathrm{~N}$ & Date and time when record was most recently updated. \\
\hline EditedBy & varchar & 64 & 0 & $\mathrm{~N}$ & $\mathrm{~N}$ & $\begin{array}{l}\text { Database username of person who most recently updated } \\
\text { record. }\end{array}$ \\
\hline FSLID & varchar & 50 & 0 & Y & Y & FocalSpeciesList record identifier. \\
\hline PopulationDesignation & varchar & 10 & 0 & $\mathrm{~N}$ & $\mathrm{~N}$ & $\begin{array}{l}\text { MultiSpecies Conservation Plan (MSCP) Population } \\
\text { designation. See luPopulationDesignation. }\end{array}$ \\
\hline SurveyDescriptionID & int & 0 & 0 & $\mathrm{Y}$ & $\mathrm{N}$ & Associated SurveyDescription record identifier. \\
\hline
\end{tabular}

Funding Table

\begin{tabular}{lcccccl}
\hline \multicolumn{1}{c}{ Field Name } & $\begin{array}{c}\text { Data } \\
\text { Type }\end{array}$ & $\begin{array}{c}\text { Field } \\
\text { Length }\end{array}$ & $\begin{array}{c}\text { Decimal } \\
\text { Places }\end{array}$ & $\begin{array}{c}\text { Required } \\
\text { Field }\end{array}$ & $\begin{array}{c}\text { Primary } \\
\text { Key }\end{array}$ & Definition \\
\hline AmountFunded & money & 19 & 4 & $\mathrm{~N}$ & $\mathrm{~N}$ & Amount awarded by grant, contract, or other funding source. \\
AwardDate & datetime & 0 & 0 & $\mathrm{~N}$ & $\mathrm{~N}$ & Date funding agreement executed.
\end{tabular}


Funding Table-Continued

\begin{tabular}{|c|c|c|c|c|c|c|}
\hline Field Name & $\begin{array}{l}\text { Data } \\
\text { Type }\end{array}$ & $\begin{array}{c}\text { Field } \\
\text { Length }\end{array}$ & $\begin{array}{c}\text { Decimal } \\
\text { Places }\end{array}$ & $\begin{array}{l}\text { Required } \\
\text { Field }\end{array}$ & $\begin{array}{c}\text { Primary } \\
\text { Key }\end{array}$ & Definition \\
\hline CreatedBy & varchar & 64 & 0 & $\mathrm{Y}$ & $\mathrm{N}$ & Database username of person who created record. \\
\hline EditDate & datetime & 0 & 0 & $\mathrm{~N}$ & $\mathrm{~N}$ & Date and time when record was most recently updated. \\
\hline EditedBy & varchar & 64 & 0 & $\mathrm{~N}$ & $\mathrm{~N}$ & Database username of person who most recently updated record. \\
\hline FundingID & bigint & 19 & 0 & $\mathrm{Y}$ & Y & Funding record identifier. \\
\hline FundingPOC & int & 0 & 0 & $\mathrm{~N}$ & $\mathrm{~N}$ & Agency Point of Contact. \\
\hline FundingProgramID & int & 0 & 0 & $\mathrm{~N}$ & $\mathrm{~N}$ & Associated FundingProgram record identifier. \\
\hline FundingTypeID & int & 0 & 0 & $\mathrm{~N}$ & $\mathrm{~N}$ & Type of funding for project. See luFundingType. \\
\hline QAQC_Note & varchar & 255 & 0 & $\mathrm{~N}$ & $\mathrm{~N}$ & Reason for change made to record during QAQC review. \\
\hline QAQC_Status & varchar & 20 & 0 & $\mathrm{Y}$ & $\mathrm{N}$ & Describes current stage of quality-analysis/quality-control. \\
\hline Recipient & int & 0 & 0 & $\mathrm{~N}$ & $\mathrm{~N}$ & Person/Agency that received the funding. \\
\hline RecipientPOC & int & 0 & 0 & $\mathrm{~N}$ & $\mathrm{~N}$ & Recipient Point of Contact. \\
\hline
\end{tabular}

\section{Funding Programs Table}

\begin{tabular}{lcrcccl}
\hline \multicolumn{1}{c}{ Field Name } & $\begin{array}{c}\text { Data } \\
\text { Type }\end{array}$ & $\begin{array}{c}\text { Field } \\
\text { Length }\end{array}$ & $\begin{array}{c}\text { Decimal } \\
\text { Places }\end{array}$ & $\begin{array}{c}\text { Required } \\
\text { Field }\end{array}$ & $\begin{array}{c}\text { Primary } \\
\text { Key }\end{array}$ & Definition \\
\hline CreatedBy & varchar & 64 & 0 & $\mathrm{Y}$ & $\mathrm{N}$ & Database username of person who created record. \\
CreatedDate & datetime & 0 & 0 & $\mathrm{Y}$ & $\mathrm{N}$ & Date and time when record was created. \\
EditDate & datetime & 0 & 0 & $\mathrm{~N}$ & $\mathrm{~N}$ & Date and time when record was most recently updated. \\
EditedBy & varchar & 64 & 0 & $\mathrm{~N}$ & $\mathrm{~N}$ & Database username of person who most recently updated record. \\
FundingEntity & int & 0 & 0 & $\mathrm{~N}$ & $\mathrm{~N}$ & Agency that awarded the grant, contract, or other funding source. \\
FundingProgramID & int & 0 & 0 & $\mathrm{Y}$ & $\mathrm{Y}$ & FundingProgram record identifier. \\
FundingTypeID & int & 0 & 0 & $\mathrm{~N}$ & $\mathrm{~N}$ & Type of funding. See luFundingType. \\
FundingYear & numeric & 4 & 0 & $\mathrm{~N}$ & $\mathrm{~N}$ & Year of funding. \\
Notes & varchar & 255 & 0 & $\mathrm{~N}$ & $\mathrm{~N}$ & Notes about the Funding Program. \\
ProgramName & varchar & 50 & 0 & $\mathrm{~N}$ & $\mathrm{~N}$ & Name of the program. \\
ProgramPOC & int & 0 & 0 & $\mathrm{~N}$ & $\mathrm{~N}$ & Program Point of Contact. See Affiliate table. \\
ProposalDueDate & datetime & 0 & 0 & $\mathrm{~N}$ & $\mathrm{~N}$ & Due date of proposal for Funding Program. \\
QAQC_Note & varchar & 255 & 0 & $\mathrm{~N}$ & $\mathrm{~N}$ & Reason for change made to record during QAQC review. \\
QAQC_Status & varchar & 20 & 0 & $\mathrm{Y}$ & $\mathrm{N}$ & Describes current stage of QAQC. \\
RFPDate & datetime & 0 & 0 & $\mathrm{~N}$ & $\mathrm{~N}$ & Due date of request for proposals (RFP). \\
\hline
\end{tabular}

\section{GrantMgtActions Table}

\begin{tabular}{|c|c|c|c|c|c|c|}
\hline Field Name & $\begin{array}{l}\text { Data } \\
\text { Type }\end{array}$ & $\begin{array}{c}\text { Field } \\
\text { Length }\end{array}$ & $\begin{array}{l}\text { Decimal } \\
\text { Places }\end{array}$ & $\begin{array}{l}\text { Required } \\
\text { Field }\end{array}$ & $\begin{array}{l}\text { Primary } \\
\text { Key }\end{array}$ & Definition \\
\hline AdminAction & int & 0 & 0 & $\mathrm{~N}$ & $\mathrm{~N}$ & $\begin{array}{l}\text { Administrative action associated with management action. See } \\
\text { luFundingAction. }\end{array}$ \\
\hline BillingAmount & money & 19 & 4 & $\mathrm{~N}$ & $\mathrm{~N}$ & Billing amount for management action. \\
\hline
\end{tabular}


GrantMgtActions Table-Continued

\begin{tabular}{lcccccl}
\hline \multicolumn{1}{c}{ Field Name } & $\begin{array}{c}\text { Data } \\
\text { Type }\end{array}$ & $\begin{array}{c}\text { Field } \\
\text { Length }\end{array}$ & $\begin{array}{c}\text { Decimal } \\
\text { Places }\end{array}$ & $\begin{array}{c}\text { Required } \\
\text { Field }\end{array}$ & $\begin{array}{c}\text { Primary } \\
\text { Key }\end{array}$ & Definition \\
\hline BillingDate & datetime & 0 & 0 & $\mathrm{~N}$ & $\mathrm{~N}$ & Billing date for management action. \\
CreatedBy & varchar & 64 & 0 & $\mathrm{Y}$ & $\mathrm{N}$ & Database username of person who created record. \\
CreatedDate & datetime & 0 & 0 & $\mathrm{Y}$ & $\mathrm{N}$ & Date and time when record was created. \\
EditDate & datetime & 0 & 0 & $\mathrm{~N}$ & $\mathrm{~N}$ & Date and time when record was most recently updated. \\
EditedBy & varchar & 64 & 0 & $\mathrm{~N}$ & $\mathrm{~N}$ & Database username of person who most recently updated record. \\
FundingID & bigint & 19 & 0 & $\mathrm{Y}$ & $\mathrm{N}$ & Associated Funding record identifier. \\
GrantMgtActionID & int & 0 & 0 & $\mathrm{Y}$ & $\mathrm{Y}$ & GrantMgtAction record identifier. \\
Notes & varchar & 255 & 0 & $\mathrm{~N}$ & $\mathrm{~N}$ & Notes about the action. \\
QAQC_Note & varchar & 255 & 0 & $\mathrm{~N}$ & $\mathrm{~N}$ & Reason for change made to record during QAQC review. \\
QAQC_Status & varchar & 20 & 0 & $\mathrm{Y}$ & $\mathrm{N}$ & Describes current stage of QAQC. \\
\hline
\end{tabular}

GrantMgtActions_Actions Table

\begin{tabular}{lcccccl}
\hline \multicolumn{1}{c}{ Field Name } & $\begin{array}{c}\text { Data } \\
\text { Type }\end{array}$ & $\begin{array}{c}\text { Field } \\
\text { Length }\end{array}$ & $\begin{array}{c}\text { Decimal } \\
\text { Places }\end{array}$ & $\begin{array}{c}\text { Required } \\
\text { Field }\end{array}$ & $\begin{array}{c}\text { Primary } \\
\text { Key }\end{array}$ & Definition \\
\hline ActionID & bigint & 19 & 0 & $\mathrm{~N}$ & $\mathrm{~N}$ & Associated Action record identifier. \\
Cost & money & 19 & 4 & $\mathrm{~N}$ & $\mathrm{~N}$ & $\begin{array}{l}\text { Total estimated cost of this action, including labor, equipment, and } \\
\text { supplies. }\end{array}$ \\
CreatedBy & varchar & 64 & 0 & $\mathrm{Y}$ & $\mathrm{N}$ & $\begin{array}{l}\text { Database username of person who created record. } \\
\text { CreatedDate }\end{array}$ \\
datetime & 0 & 0 & $\mathrm{Y}$ & $\mathrm{N}$ & Date and time when record was created. \\
EditDate & datetime & 0 & 0 & $\mathrm{~N}$ & $\mathrm{~N}$ & Date and time when record was most recently updated. \\
EditedBy & varchar & 64 & 0 & $\mathrm{~N}$ & $\mathrm{~N}$ & Database username of person who most recently updated record. \\
GMAAID & int & 0 & 0 & $\mathrm{Y}$ & $\mathrm{Y}$ & GrantMgtAction_Action record identifier. \\
GrantMgtActionID & int & 0 & 0 & $\mathrm{~N}$ & $\mathrm{~N}$ & Associated GrantMgtAction record identifier. \\
Hours & decimal & 7 & 2 & $\mathrm{~N}$ & $\mathrm{~N}$ & Total estimated labor hours to complete this action. \\
Notes & varchar & 200 & 0 & $\mathrm{~N}$ & $\mathrm{~N}$ & Notes about the action. \\
\hline & & & & & &
\end{tabular}

HabitatObservation Table

\begin{tabular}{|c|c|c|c|c|c|c|}
\hline Field Name & $\begin{array}{l}\text { Data } \\
\text { Type }\end{array}$ & $\begin{array}{l}\text { Field } \\
\text { Length }\end{array}$ & $\begin{array}{l}\text { Decimal } \\
\text { Places }\end{array}$ & $\begin{array}{l}\text { Required } \\
\text { Field }\end{array}$ & $\begin{array}{l}\text { Primary } \\
\text { Key }\end{array}$ & Definition \\
\hline AspectDirection Code1 & varchar & 3 & 0 & $\mathrm{~N}$ & $\mathrm{~N}$ & First most dominant aspect direction at the site. \\
\hline $\begin{array}{l}\text { BankDisturbance } \\
\text { Evidence }\end{array}$ & varchar & 100 & 0 & $\mathrm{~N}$ & $\mathrm{~N}$ & Description of evidence suggesting bank disturbance. \\
\hline $\begin{array}{l}\text { BeaverActivity } \\
\text { Evidence }\end{array}$ & varchar & 100 & 0 & $\mathrm{~N}$ & $\mathrm{~N}$ & Description of evidence suggesting beaver activity. \\
\hline Created & datetime & 0 & 0 & $\mathrm{~N}$ & $\mathrm{~N}$ & Pendragon PDA field, for PDA compatibility. \\
\hline CreatedBy & varchar & 64 & 0 & $\mathrm{Y}$ & $\mathrm{N}$ & Database username of person who created record. \\
\hline CreatedDate & datetime & 0 & 0 & $\mathrm{Y}$ & $\mathrm{N}$ & Date and time when record was created. \\
\hline DistanceToRoad & int & 0 & 0 & $\mathrm{~N}$ & $\mathrm{~N}$ & Distance in meters to the nearest road. \\
\hline DistanceToWater & decimal & 8 & 2 & $\mathrm{~N}$ & $\mathrm{~N}$ & Distance to nearest water source, measured in meters. \\
\hline EditDate & datetime & 0 & 0 & $\mathrm{~N}$ & $\mathrm{~N}$ & Date and time when record was most recently updated. \\
\hline EditedBy & varchar & 64 & 0 & $\mathrm{~N}$ & $\mathrm{~N}$ & $\begin{array}{l}\text { Database username of person who most recently updated } \\
\text { record. }\end{array}$ \\
\hline EditNotes & varchar & 50 & 0 & $\mathrm{~N}$ & $\mathrm{~N}$ & Notes about the updated record. \\
\hline Elevation & int & 0 & 0 & $\mathrm{~N}$ & $\mathrm{~N}$ & Elevation, in meters, of habitat. \\
\hline
\end{tabular}




\section{HabitatObservation Table-Continued}

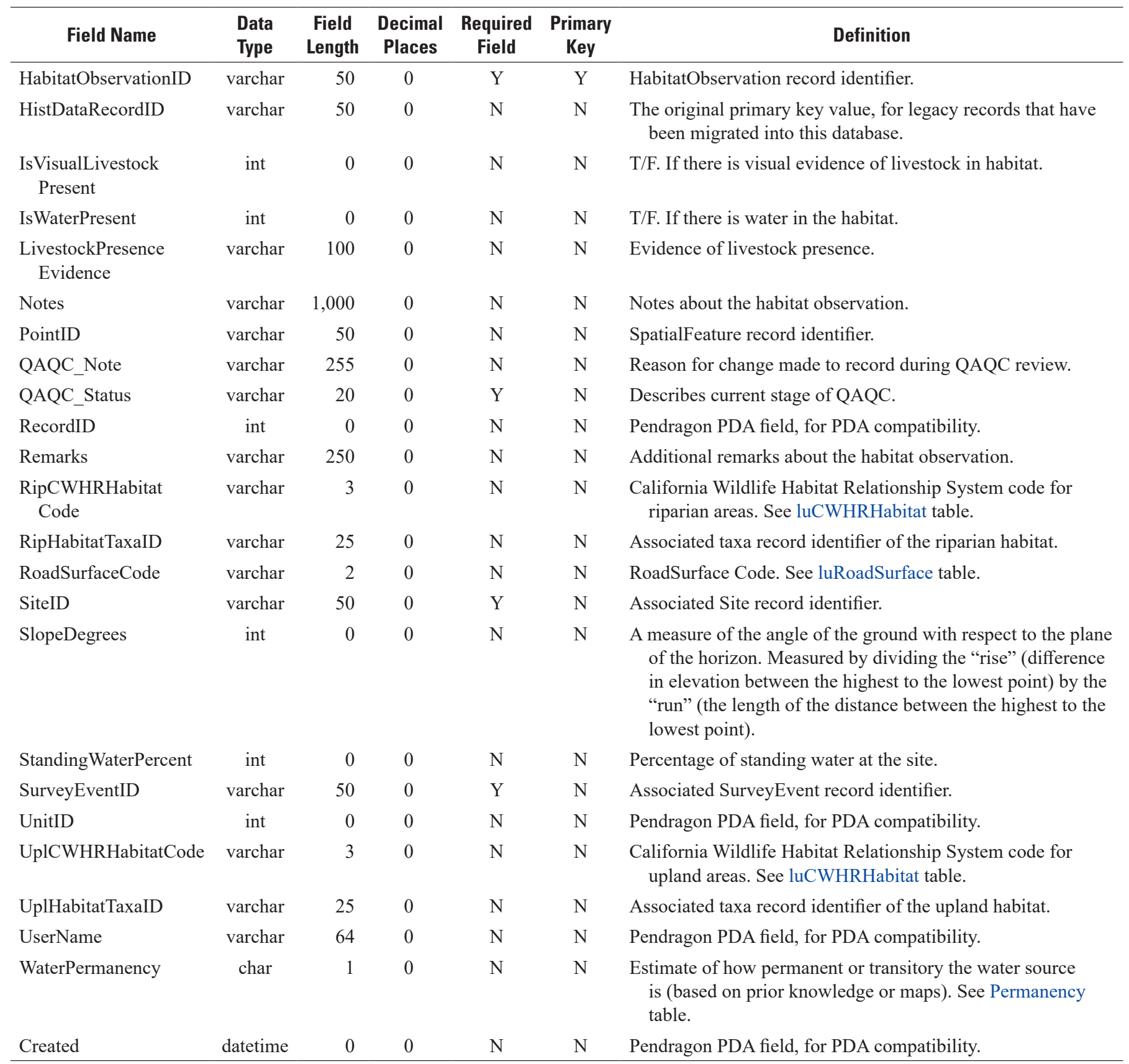

\section{HabitatObservation_Feature Table}

\section{Field Name}

\section{CreatedBy}

CreatedDate

EditDate

EditedBy

$\begin{array}{ccccc}\begin{array}{c}\text { Data } \\ \text { Type }\end{array} & \begin{array}{c}\text { Field } \\ \text { Length }\end{array} & \begin{array}{c}\text { Decimal } \\ \text { Places }\end{array} & \begin{array}{c}\text { Required } \\ \text { Field }\end{array} & \begin{array}{c}\text { Primary } \\ \text { Key }\end{array} \\ \text { varchar } & 64 & 0 & \mathrm{Y} & \mathrm{N} \\ \text { datetime } & 0 & 0 & \mathrm{Y} & \mathrm{N} \\ \text { datetime } & 0 & 0 & \mathrm{~N} & \mathrm{~N} \\ \text { varchar } & 64 & 0 & \mathrm{~N} & \mathrm{~N}\end{array}$

\section{Definition}

Database username of person who created record.

Date and time when record was created.

Date and time when record was most recently updated.

Database username of person who most recently updated record. 
HabitatObservation Feature Table-Continued

\begin{tabular}{lcccccc}
\hline \multicolumn{1}{c}{ Field Name } & $\begin{array}{c}\text { Data } \\
\text { Type }\end{array}$ & $\begin{array}{c}\text { Field } \\
\text { Length }\end{array}$ & $\begin{array}{c}\text { Decimal } \\
\text { Places }\end{array}$ & $\begin{array}{c}\text { Required } \\
\text { Field }\end{array}$ & $\begin{array}{c}\text { Primary } \\
\text { Key }\end{array}$ & Definition \\
\hline $\begin{array}{l}\text { HabitatFeatureType } \\
\text { ID }\end{array}$ & int & 0 & 0 & Y & Y & $\begin{array}{c}\text { Type of feature observed within habitat. Used to record } \\
\text { features of note within a habitat (for example, provides } \\
\text { beneficial habitat, survey waypoints, and so forth). See } \\
\text { luHabitatFeatureType table. }\end{array}$ \\
HabitatObservationID & varchar & 50 & 0 & Y & Y & Associated HabitatObservation record identifier. \\
NumFeatures & int & 0 & 0 & $\mathrm{~N}$ & $\mathrm{~N}$ & Number of features observed. \\
RecordID & int & 0 & 0 & $\mathrm{~N}$ & $\mathrm{~N}$ & Pendragon PDA field, for PDA compatibility. \\
UnitID & int & 0 & 0 & $\mathrm{~N}$ & $\mathrm{~N}$ & Pendragon PDA field, for PDA compatibility. \\
UserName & varchar & 64 & 0 & $\mathrm{~N}$ & $\mathrm{~N}$ & Pendragon PDA field, for PDA compatibility. \\
\hline
\end{tabular}

HabitatObservation_GroundCover Table

\begin{tabular}{lcccccl}
\hline \multicolumn{1}{c}{ Field Name } & $\begin{array}{c}\text { Data } \\
\text { Type }\end{array}$ & $\begin{array}{c}\text { Field } \\
\text { Length }\end{array}$ & $\begin{array}{c}\text { Decimal } \\
\text { Places }\end{array}$ & $\begin{array}{c}\text { Required } \\
\text { Field }\end{array}$ & $\begin{array}{c}\text { Primary } \\
\text { Key }\end{array}$ & Definition \\
\hline Created & datetime & 0 & 0 & $\mathrm{~N}$ & $\mathrm{~N}$ & Pendragon PDA field, for PDA compatibility. \\
CreatedBy & varchar & 64 & 0 & $\mathrm{Y}$ & $\mathrm{N}$ & Database username of person who created record. \\
CreatedDate & datetime & 0 & 0 & $\mathrm{Y}$ & $\mathrm{N}$ & Date and time when record was created. \\
EditDate & datetime & 0 & 0 & $\mathrm{~N}$ & $\mathrm{~N}$ & Date and time when record was most recently updated. \\
EditedBy & varchar & 64 & 0 & $\mathrm{~N}$ & $\mathrm{~N}$ & $\begin{array}{c}\text { Database username of person who most recently updated } \\
\text { record. }\end{array}$ \\
GroundCoverID & int & 0 & 0 & $\mathrm{Y}$ & $\mathrm{Y}$ & $\begin{array}{c}\text { Type of ground cover observed within habitat. See } \\
\text { GroundCoverPercent }\end{array}$ \\
HabitatObservationID & varchar & 50 & 0 & $\mathrm{Y}$ & $\mathrm{Y}$ & Associated HabitatObservation record identifier. \\
RecordID & int & 0 & 0 & $\mathrm{~N}$ & $\mathrm{~N}$ & Pendragon PDA field, for PDA compatibility. \\
UnitID & int & 0 & 0 & $\mathrm{~N}$ & $\mathrm{~N}$ & Pendragon PDA field, for PDA compatibility. \\
UserName & varchar & 64 & 0 & $\mathrm{~N}$ & $\mathrm{~N}$ & Pendragon PDA field, for PDA compatibility. \\
\hline
\end{tabular}

HabitatObservation_LandUse Table

\begin{tabular}{|c|c|c|c|c|c|c|}
\hline Field Name & $\begin{array}{l}\text { Data } \\
\text { Type }\end{array}$ & $\begin{array}{c}\text { Field } \\
\text { Length }\end{array}$ & $\begin{array}{c}\text { Decimal } \\
\text { Places }\end{array}$ & $\begin{array}{l}\text { Required } \\
\text { Field }\end{array}$ & $\begin{array}{c}\text { Primary } \\
\text { Key }\end{array}$ & Definition \\
\hline Created & datetime & 0 & 0 & $\mathrm{~N}$ & $\mathrm{~N}$ & Pendragon PDA field, for PDA compatibility. \\
\hline CreatedDate & datetime & 0 & 0 & $\mathrm{Y}$ & $\mathrm{N}$ & Date and time when record was created. \\
\hline EditDate & datetime & 0 & 0 & $\mathrm{~N}$ & $\mathrm{~N}$ & Date and time when record was most recently updated. \\
\hline HabitatObservationID & varchar & 50 & 0 & $\mathrm{Y}$ & $\mathrm{Y}$ & Associated HabitatObservation record identifier. \\
\hline LandUseID & int & 0 & 0 & $\mathrm{Y}$ & $\mathrm{Y}$ & The primary function of the land. See luLandUse table. \\
\hline LandUsePercent & char & 10 & 0 & $\mathrm{~N}$ & $\mathrm{~N}$ & Percentage of habitat with identified land use. \\
\hline RecordID & int & 0 & 0 & $\mathrm{~N}$ & $\mathrm{~N}$ & Pendragon PDA field, for PDA compatibility. \\
\hline
\end{tabular}


HabitatObservation_SedChemical Table

\begin{tabular}{|c|c|c|c|c|c|c|}
\hline Field Name & $\begin{array}{l}\text { Data } \\
\text { Type }\end{array}$ & $\begin{array}{l}\text { Field } \\
\text { Length }\end{array}$ & $\begin{array}{l}\text { Decimal } \\
\text { Places }\end{array}$ & $\begin{array}{l}\text { Required } \\
\text { Field }\end{array}$ & $\begin{array}{l}\text { Primary } \\
\text { Key }\end{array}$ & Definition \\
\hline $\begin{array}{l}\text { Chemical } \\
\text { Concentration }\end{array}$ & decimal & 10 & 6 & $\mathrm{~N}$ & $\mathrm{~N}$ & Concentration of the chemical in the sediment. \\
\hline Created & datetime & 0 & 0 & $\mathrm{~N}$ & $\mathrm{~N}$ & Pendragon PDA field, for PDA compatibility. \\
\hline CreatedBy & varchar & 64 & 0 & $\mathrm{Y}$ & $\mathrm{N}$ & Database username of person who created record. \\
\hline CreatedDate & datetime & 0 & 0 & $\mathrm{Y}$ & $\mathrm{N}$ & Date and time when record was created. \\
\hline EditedBy & varchar & 64 & 0 & $\mathrm{~N}$ & $\mathrm{~N}$ & $\begin{array}{l}\text { Database username of person who most recently updated } \\
\text { record. }\end{array}$ \\
\hline HabitatObservationID & varchar & 50 & 0 & $\mathrm{Y}$ & $\mathrm{Y}$ & Associated HabitatObservation record identifier. \\
\hline RecordID & int & 0 & 0 & $\mathrm{~N}$ & $\mathrm{~N}$ & Pendragon PDA field, for PDA compatibility. \\
\hline
\end{tabular}

HabitatObservation_Substrate Table

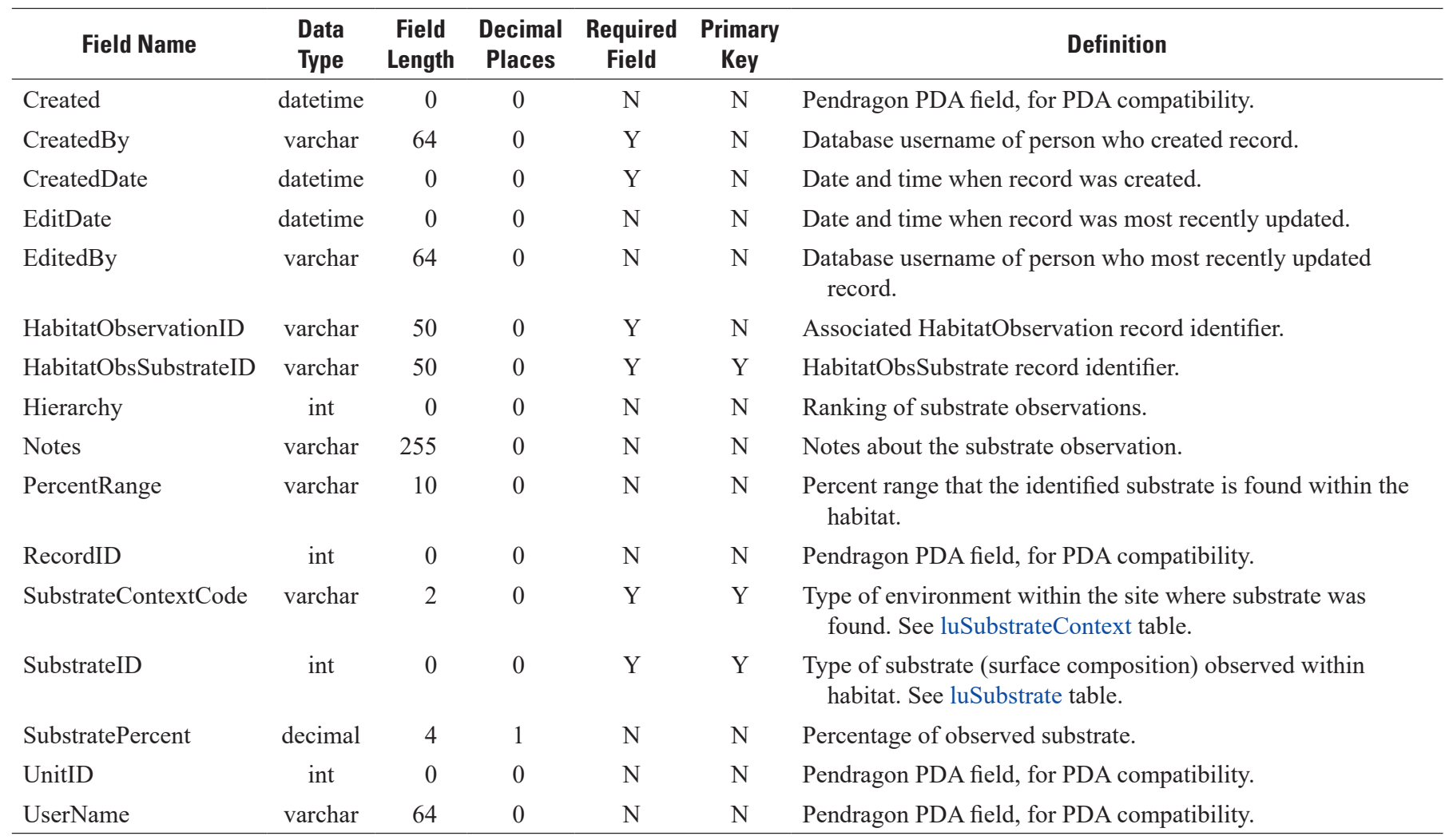


HabitatObservation_Vegetation Table

\begin{tabular}{|c|c|c|c|c|c|c|}
\hline Field Name & $\begin{array}{l}\text { Data } \\
\text { Type }\end{array}$ & $\begin{array}{c}\text { Field } \\
\text { Length }\end{array}$ & $\begin{array}{l}\text { Decimal } \\
\text { Places }\end{array}$ & $\begin{array}{l}\text { Required } \\
\text { Field }\end{array}$ & $\begin{array}{l}\text { Primary } \\
\text { Key }\end{array}$ & Definition \\
\hline Context & varchar & 20 & 0 & $\mathrm{~N}$ & $\mathrm{~N}$ & $\begin{array}{l}\text { General area of habitat in which vegetation was observed. See } \\
\text { luHabitatObsVegContext table. }\end{array}$ \\
\hline Created & datetime & 0 & 0 & $\mathrm{~N}$ & $\mathrm{~N}$ & Pendragon PDA field, for PDA compatibility. \\
\hline CreatedBy & varchar & 64 & 0 & $\mathrm{Y}$ & $\mathrm{N}$ & Database username of person who created record. \\
\hline CreatedDate & datetime & 0 & 0 & $\mathrm{Y}$ & $\mathrm{N}$ & Date and time when record was created. \\
\hline EditedBy & varchar & 64 & 0 & $\mathrm{~N}$ & $\mathrm{~N}$ & Database username of person who most recently updated record. \\
\hline $\begin{array}{l}\text { HabitatObservation } \\
\quad \text { ID }\end{array}$ & varchar & 50 & 0 & Y & $\mathrm{N}$ & Associated HabitatObservation record identifier. \\
\hline HabitatObsVegID & varchar & 50 & 0 & Y & Y & HabitatObs_Vegetation record identifier. \\
\hline $\begin{array}{l}\text { HabitatVegetation } \\
\text { TypeID }\end{array}$ & int & 0 & 0 & $\mathrm{Y}$ & Y & $\begin{array}{l}\text { Type of vegetation found within habitat. See } \\
\text { luHabitatVegetationType table. }\end{array}$ \\
\hline UserName & varchar & 64 & 0 & $\mathrm{~N}$ & $\mathrm{~N}$ & Pendragon PDA field, for PDA compatibility. \\
\hline $\begin{array}{l}\text { VegetationMax } \\
\text { Height }\end{array}$ & decimal & 10 & 4 & $\mathrm{~N}$ & $\mathrm{~N}$ & Maximum height of observed vegetation. \\
\hline $\begin{array}{l}\text { VegetationType } \\
\text { Percent }\end{array}$ & int & 0 & 0 & $\mathrm{~N}$ & $\mathrm{~N}$ & Percentage of identified vegetation observed within habitat. \\
\hline $\begin{array}{l}\text { VegType } \\
\text { PercentRange }\end{array}$ & varchar & 10 & 0 & $\mathrm{~N}$ & $\mathrm{~N}$ & Range (min to $\max$ ) in percentages of the vegetation type. \\
\hline
\end{tabular}

IndivAnimals Table

\begin{tabular}{|c|c|c|c|c|c|c|}
\hline Field Name & $\begin{array}{l}\text { Data } \\
\text { Type }\end{array}$ & $\begin{array}{l}\text { Field } \\
\text { Length }\end{array}$ & $\begin{array}{l}\text { Decimal } \\
\text { Places }\end{array}$ & $\begin{array}{l}\text { Required } \\
\text { Field }\end{array}$ & $\begin{array}{l}\text { Primary } \\
\text { Key }\end{array}$ & Definition \\
\hline AbnormalDesc & varchar & 200 & 0 & $\mathrm{~N}$ & $\mathrm{~N}$ & Description of abnormalities observed on animal. \\
\hline AgeDetMethod & varchar & 50 & 0 & $\mathrm{~N}$ & $\mathrm{~N}$ & Description of method used to determine age of individual animal. \\
\hline Atmarg & numeric & 2 & 0 & $\mathrm{~N}$ & $\mathrm{~N}$ & $\begin{array}{l}\text { The number of the marginal scute (scute/scalel located along } \\
\text { the edge of a turtle's carapace) where the greatest width was } \\
\text { measured. }\end{array}$ \\
\hline AtmargWidth & numeric & 4 & 0 & $\mathrm{~N}$ & $\mathrm{~N}$ & Width of carapace at the Atmarg location. \\
\hline BodyGirth & int & 0 & 0 & $\mathrm{~N}$ & $\mathrm{~N}$ & Distance around the animal's body at the widest point. \\
\hline BodyLength & decimal & 5 & 1 & $\mathrm{~N}$ & $\mathrm{~N}$ & $\begin{array}{l}\text { For anurans, this is measured from tip of the snout to the tip of the } \\
\text { urostyle, unless otherwise noted in protocol and/or metadata. }\end{array}$ \\
\hline BodyTemp & decimal & 5 & 1 & $\mathrm{~N}$ & $\mathrm{~N}$ & Body temperature of the animal in Celsius. \\
\hline CementumRidge & int & 0 & 0 & $\mathrm{~N}$ & $\mathrm{~N}$ & Measurement of ridge in millimeters. \\
\hline ChestCirc & decimal & 5 & 1 & $\mathrm{~N}$ & $\mathrm{~N}$ & Circumference of chest in $\mathrm{mm}$. \\
\hline Created & datetime & 0 & 0 & $\mathrm{~N}$ & $\mathrm{~N}$ & Pendragon PDA field, for PDA compatibility. \\
\hline CreatedBy & varchar & 64 & 0 & Y & $\mathrm{N}$ & Database username of person who created record. \\
\hline CreatedDate & datetime & 0 & 0 & Y & $\mathrm{N}$ & Date and time when record was created. \\
\hline Disposition & varchar & 10 & 0 & $\mathrm{~N}$ & $\mathrm{~N}$ & $\begin{array}{l}\text { Disposition of animal when observed (for example, alive, dead). } \\
\text { See luDisposition table. }\end{array}$ \\
\hline
\end{tabular}




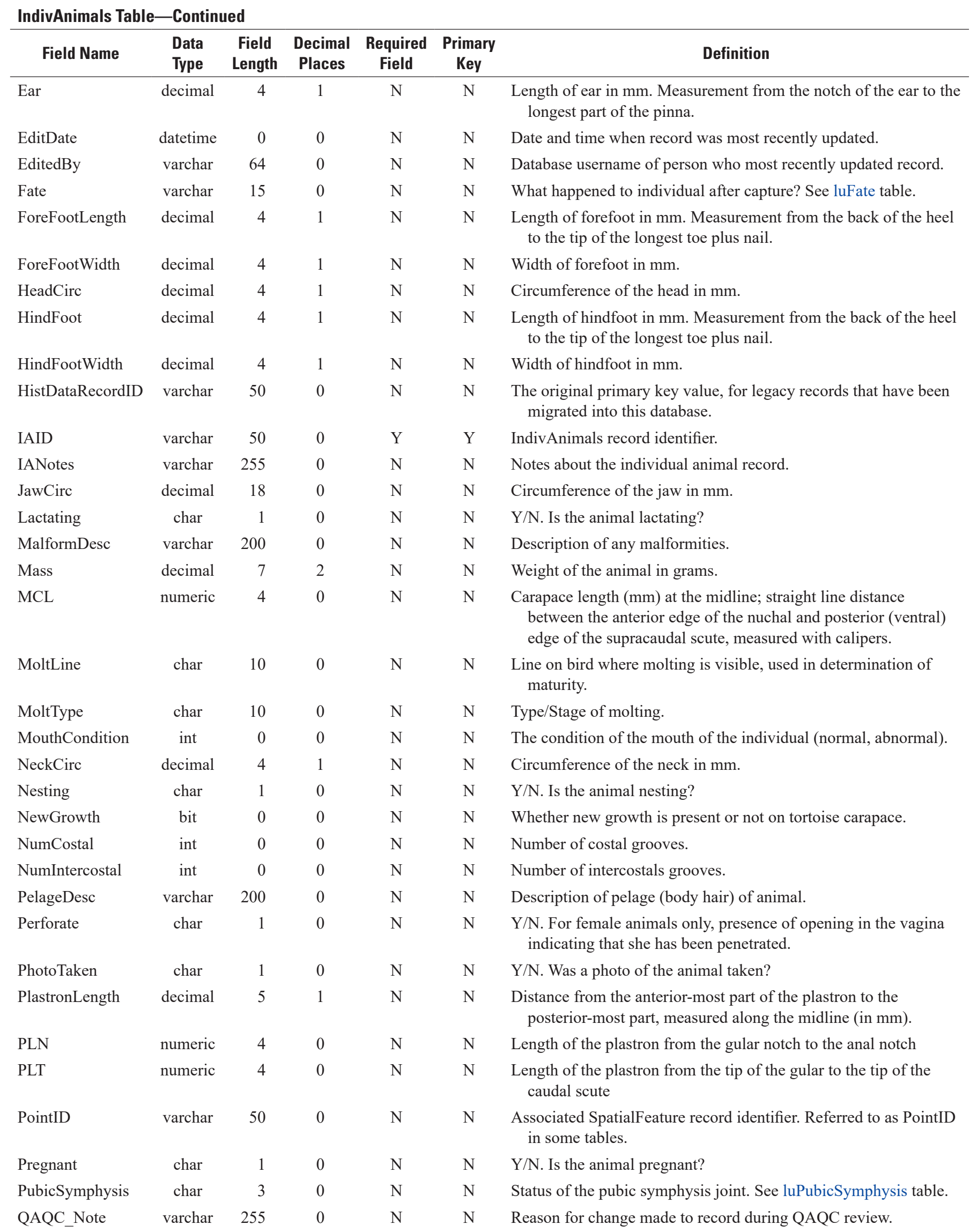


IndivAnimals Table-Continued

\begin{tabular}{|c|c|c|c|c|c|c|}
\hline Field Name & $\begin{array}{l}\text { Data } \\
\text { Type }\end{array}$ & $\begin{array}{c}\text { Field } \\
\text { Length }\end{array}$ & $\begin{array}{l}\text { Decimal } \\
\text { Places }\end{array}$ & $\begin{array}{l}\text { Required } \\
\text { Field }\end{array}$ & $\begin{array}{l}\text { Primary } \\
\text { Key }\end{array}$ & Definition \\
\hline QAQC_Status & varchar & 20 & 0 & $\mathrm{Y}$ & $\mathrm{N}$ & Describes current stage of QAQC. \\
\hline RecordID & int & 0 & 0 & $\mathrm{~N}$ & $\mathrm{~N}$ & Pendragon PDA field, for PDA compatibility. \\
\hline Residency & char & 1 & 0 & $\mathrm{~N}$ & $\mathrm{~N}$ & Residency condition (wintering, territory, rookery). \\
\hline Sex & int & 0 & 0 & $\mathrm{~N}$ & $\mathrm{~N}$ & Sex of the animal. \\
\hline Shwear & numeric & 1 & 0 & $\mathrm{~N}$ & $\mathrm{~N}$ & Degree of shell wear observed on tortoise shell. See luShwear. \\
\hline SpermPlug & char & 1 & 0 & $\mathrm{~N}$ & $\mathrm{~N}$ & $\begin{array}{l}\text { Y/N. For female animals only, presence of sperm plug (gooey } \\
\text { film) over the vagina after penetration. }\end{array}$ \\
\hline TailLength & decimal & 5 & 1 & $\mathrm{~N}$ & $\mathrm{~N}$ & $\begin{array}{l}\text { Tail length of the individual animal in mm, measurement from } \\
\text { the base of the rump to the tip of the last vertebrate. Hair is not } \\
\text { included. }\end{array}$ \\
\hline Teats & char & 2 & 0 & $\mathrm{~N}$ & $\mathrm{~N}$ & Condition of teats. See luTeats table. \\
\hline TissueSample & char & 1 & 0 & $\mathrm{~N}$ & $\mathrm{~N}$ & Y/N. Whether tissue sample was taken. \\
\hline TotalLength & decimal & 5 & 1 & $\mathrm{~N}$ & $\mathrm{~N}$ & $\begin{array}{l}\text { Total length of the individual animal in mm, measured from } \\
\text { beginning of snout to tip of tail. }\end{array}$ \\
\hline UnitID & int & 0 & 0 & $\mathrm{~N}$ & $\mathrm{~N}$ & Pendragon PDA field, for PDA compatibility. \\
\hline UserName & varchar & 64 & 0 & $\mathrm{~N}$ & $\mathrm{~N}$ & Pendragon PDA field, for PDA compatibility. \\
\hline Width_M3 & numeric & 4 & 0 & $\mathrm{~N}$ & $\mathrm{~N}$ & $\begin{array}{l}\text { Width of the tortoise at the posterior edge of the third marginal } \\
\text { scute. }\end{array}$ \\
\hline
\end{tabular}

IndividualAnimalldentifier Table

\begin{tabular}{lcccccl}
\hline \multicolumn{1}{c}{ Field Name } & $\begin{array}{c}\text { Data } \\
\text { Type }\end{array}$ & $\begin{array}{c}\text { Field } \\
\text { Length }\end{array}$ & $\begin{array}{c}\text { Decimal } \\
\text { Places }\end{array}$ & $\begin{array}{c}\text { Required } \\
\text { Field }\end{array}$ & $\begin{array}{c}\text { Primary } \\
\text { Key }\end{array}$ & Definition \\
\hline Created & datetime & 0 & 0 & $\mathrm{~N}$ & $\mathrm{~N}$ & Pendragon PDA field, for PDA compatibility. \\
CreatedBy & varchar & 64 & 0 & $\mathrm{Y}$ & $\mathrm{N}$ & Database username of person who created record. \\
CreatedDate & datetime & 0 & 0 & $\mathrm{Y}$ & $\mathrm{N}$ & Date and time when record was created. \\
EditDate & datetime & 0 & 0 & $\mathrm{~N}$ & $\mathrm{~N}$ & Date and time when record was most recently updated. \\
EditedBy & varchar & 64 & 0 & $\mathrm{~N}$ & $\mathrm{~N}$ & Database username of person who most recently updated record. \\
IAID & varchar & 50 & 0 & $\mathrm{~N}$ & $\mathrm{~N}$ & Associated IndivAnimal record identifier. \\
IDType & char & 10 & 0 & $\mathrm{~N}$ & $\mathrm{~N}$ & Type of tag or identification marker used on animal. See IDType \\
& & & & & & table. \\
IDValue & varchar & 250 & 0 & $\mathrm{~N}$ & $\mathrm{~N}$ & Identification number of animal/tag. \\
IndivAnimalIDID & varchar & 50 & 0 & $\mathrm{Y}$ & $\mathrm{Y}$ & IndividualAnimalIdentifier record identifier. \\
IsChange & char & 1 & 0 & $\mathrm{~N}$ & $\mathrm{~N}$ & Y/N. Is the current IDValue a new value? \\
Notes & varchar & 200 & 0 & $\mathrm{~N}$ & $\mathrm{~N}$ & Notes about the animal identifier. \\
OldIDType & char & 10 & 0 & $\mathrm{~N}$ & $\mathrm{~N}$ & Former type of tag or identification marker used on animal. See \\
& & & & & & IDType table.
\end{tabular}


IndividualAnimalldentifier Table-Continued

\begin{tabular}{lcccccl}
\hline \multicolumn{1}{c}{ Field Name } & $\begin{array}{c}\text { Data } \\
\text { Type }\end{array}$ & $\begin{array}{c}\text { Field } \\
\text { Length }\end{array}$ & $\begin{array}{c}\text { Decimal } \\
\text { Places }\end{array}$ & $\begin{array}{c}\text { Required } \\
\text { Field }\end{array}$ & $\begin{array}{c}\text { Primary } \\
\text { Key }\end{array}$ & Definition \\
\hline OldIDValue & varchar & 250 & 0 & $\mathrm{~N}$ & $\mathrm{~N}$ & Former identification number of animal/tag. \\
RecordID & int & 0 & 0 & $\mathrm{~N}$ & $\mathrm{~N}$ & Pendragon PDA field, for PDA compatibility. \\
UnitID & int & 0 & 0 & $\mathrm{~N}$ & $\mathrm{~N}$ & Pendragon PDA field, for PDA compatibility. \\
UserName & varchar & 64 & 0 & $\mathrm{~N}$ & $\mathrm{~N}$ & Pendragon PDA field, for PDA compatibility. \\
\hline
\end{tabular}

Keywords Table

\begin{tabular}{lcccccc}
\hline Field Name & $\begin{array}{c}\text { Data } \\
\text { Type }\end{array}$ & $\begin{array}{c}\text { Field } \\
\text { Length }\end{array}$ & $\begin{array}{c}\text { Decimal } \\
\text { Places }\end{array}$ & $\begin{array}{c}\text { Required } \\
\text { Field }\end{array}$ & $\begin{array}{c}\text { Primary } \\
\text { Key }\end{array}$ & Definition \\
\hline CreatedBy & varchar & 64 & 0 & $\mathrm{Y}$ & $\mathrm{N}$ & Database username of person who created record. \\
CreatedDate & datetime & 0 & 0 & $\mathrm{Y}$ & $\mathrm{N}$ & Date and time when record was created. \\
EditDate & datetime & 0 & 0 & $\mathrm{~N}$ & $\mathrm{~N}$ & Date and time when record was most recently updated. \\
EditedBy & varchar & 64 & 0 & $\mathrm{~N}$ & $\mathrm{~N}$ & Database username of person who most recently updated record. \\
Keyword & varchar & 50 & 0 & $\mathrm{Y}$ & $\mathrm{N}$ & Keyword. \\
KeywordID & bigint & 19 & 0 & $\mathrm{Y}$ & $\mathrm{Y}$ & Keywords record identifier. \\
Type & varchar & 25 & 0 & $\mathrm{~N}$ & $\mathrm{~N}$ & $\begin{array}{l}\text { Optional way to classify and/or organize the keywords into lists, and so } \\
\text { forth. See luKeywordType. }\end{array}$ \\
\hline
\end{tabular}

Lab Results Table

\begin{tabular}{lcccccl}
\hline \multicolumn{1}{c}{ Field Name } & $\begin{array}{c}\text { Data } \\
\text { Type }\end{array}$ & $\begin{array}{c}\text { Field } \\
\text { Length }\end{array}$ & $\begin{array}{c}\text { Decimal } \\
\text { Places }\end{array}$ & $\begin{array}{c}\text { Required } \\
\text { Field }\end{array}$ & $\begin{array}{c}\text { Primary } \\
\text { Key }\end{array}$ & Definition \\
\hline AnalysisDate & date & 0 & 0 & $\mathrm{~N}$ & $\mathrm{~N}$ & Date the lab analysis was conducted. \\
CollectionID & varchar & 50 & 0 & $\mathrm{~N}$ & $\mathrm{~N}$ & Associated Collection record identifier. \\
CreatedBy & varchar & 64 & 0 & $\mathrm{Y}$ & $\mathrm{N}$ & Database username of person who created record. \\
CreatedDate & datetime & 0 & 0 & $\mathrm{Y}$ & $\mathrm{N}$ & Date and time when record was created. \\
$\mathrm{Ct}$ & decimal & 6 & 3 & $\mathrm{~N}$ & $\mathrm{~N}$ & Count. \\
DNACopiesCount & decimal & 17 & 7 & $\mathrm{~N}$ & $\mathrm{~N}$ & Number of copies of DNA. \\
EditDate & datetime & 0 & 0 & $\mathrm{~N}$ & $\mathrm{~N}$ & Date and time when record was most recently updated. \\
EditedBy & varchar & 64 & 0 & $\mathrm{~N}$ & $\mathrm{~N}$ & Database username of person who most recently updated record. \\
LabID & int & 0 & 0 & $\mathrm{~N}$ & $\mathrm{~N}$ & AffiliateID of the Lab that conducted the analysis. \\
LabRefID & varchar & 30 & 0 & $\mathrm{~N}$ & $\mathrm{~N}$ & ID that lab assigned to sample. \\
LabResultsID & varchar & 50 & 0 & $\mathrm{Y}$ & $\mathrm{Y}$ & LabResults record identifier. \\
Notes & varchar & 250 & 0 & $\mathrm{~N}$ & $\mathrm{~N}$ & Notes about the lab results. \\
Result & varchar & 50 & 0 & $\mathrm{~N}$ & $\mathrm{~N}$ & Result value from analysis. \\
Test & varchar & 50 & 0 & $\mathrm{~N}$ & $\mathrm{~N}$ & Test performed by lab. \\
Trial & int & 0 & 0 & $\mathrm{~N}$ & $\mathrm{~N}$ & For tests that are run multiple times, this is the number representing \\
& & & & & & to which test in the sequence of tests this result is associated. \\
\hline
\end{tabular}

LandManagement Table

\begin{tabular}{lcccccl}
\hline \multicolumn{1}{c}{ Field Name } & $\begin{array}{c}\text { Data } \\
\text { Type }\end{array}$ & $\begin{array}{c}\text { Field } \\
\text { Length }\end{array}$ & $\begin{array}{c}\text { Decimal } \\
\text { Places }\end{array}$ & $\begin{array}{c}\text { Required } \\
\text { Field }\end{array}$ & $\begin{array}{c}\text { Primary } \\
\text { Key }\end{array}$ & Definition \\
\hline AffiliateID & int & 0 & 0 & $\mathrm{~N}$ & $\mathrm{~N}$ & Associated Affiliate record identifier. \\
CreatedBy & varchar & 64 & 0 & $\mathrm{Y}$ & $\mathrm{N}$ & Database username of person who created record. \\
CreatedDate & datetime & 0 & 0 & $\mathrm{Y}$ & $\mathrm{N}$ & Date and time when record was created.
\end{tabular}


LandManagement Table-Continued

\begin{tabular}{lcccccl}
\hline \multicolumn{1}{c}{ Field Name } & $\begin{array}{c}\text { Data } \\
\text { Type }\end{array}$ & $\begin{array}{c}\text { Field } \\
\text { Length }\end{array}$ & $\begin{array}{c}\text { Decimal } \\
\text { Places }\end{array}$ & $\begin{array}{c}\text { Required } \\
\text { Field }\end{array}$ & $\begin{array}{c}\text { Primary } \\
\text { Key }\end{array}$ & Definition \\
\hline EditDate & datetime & 0 & 0 & $\mathrm{~N}$ & $\mathrm{~N}$ & Date and time when record was most recently updated. \\
EditedBy & varchar & 64 & 0 & $\mathrm{~N}$ & $\mathrm{~N}$ & Database username of person who most recently updated record. \\
LandManagementID & varchar & 50 & 0 & $\mathrm{Y}$ & $\mathrm{Y}$ & LandManagement record identifier. \\
ManagementEnd & datetime & 0 & 0 & $\mathrm{~N}$ & $\mathrm{~N}$ & Date management of this site by this affiliate ended. \\
ManagementStart & datetime & 0 & 0 & $\mathrm{~N}$ & $\mathrm{~N}$ & Date management of this site by this affiliate began. \\
Notes & varchar & 100 & 0 & $\mathrm{~N}$ & $\mathrm{~N}$ & Notes about the Land Management record. \\
QAQC_Note & varchar & 255 & 0 & $\mathrm{~N}$ & $\mathrm{~N}$ & Reason for change made to record during QAQC review. \\
QAQC_Status & varchar & 20 & 0 & $\mathrm{Y}$ & $\mathrm{N}$ & Describes current stage of QAQC. \\
Role & varchar & 50 & 0 & $\mathrm{~N}$ & $\mathrm{~N}$ & Describes the role the affiliate plays in the management of the \\
& & & & & & site. \\
SiteID & varchar & 50 & 0 & $\mathrm{Y}$ & $\mathrm{N}$ & Associated Site record identifier.
\end{tabular}

\section{Maintenance Table}

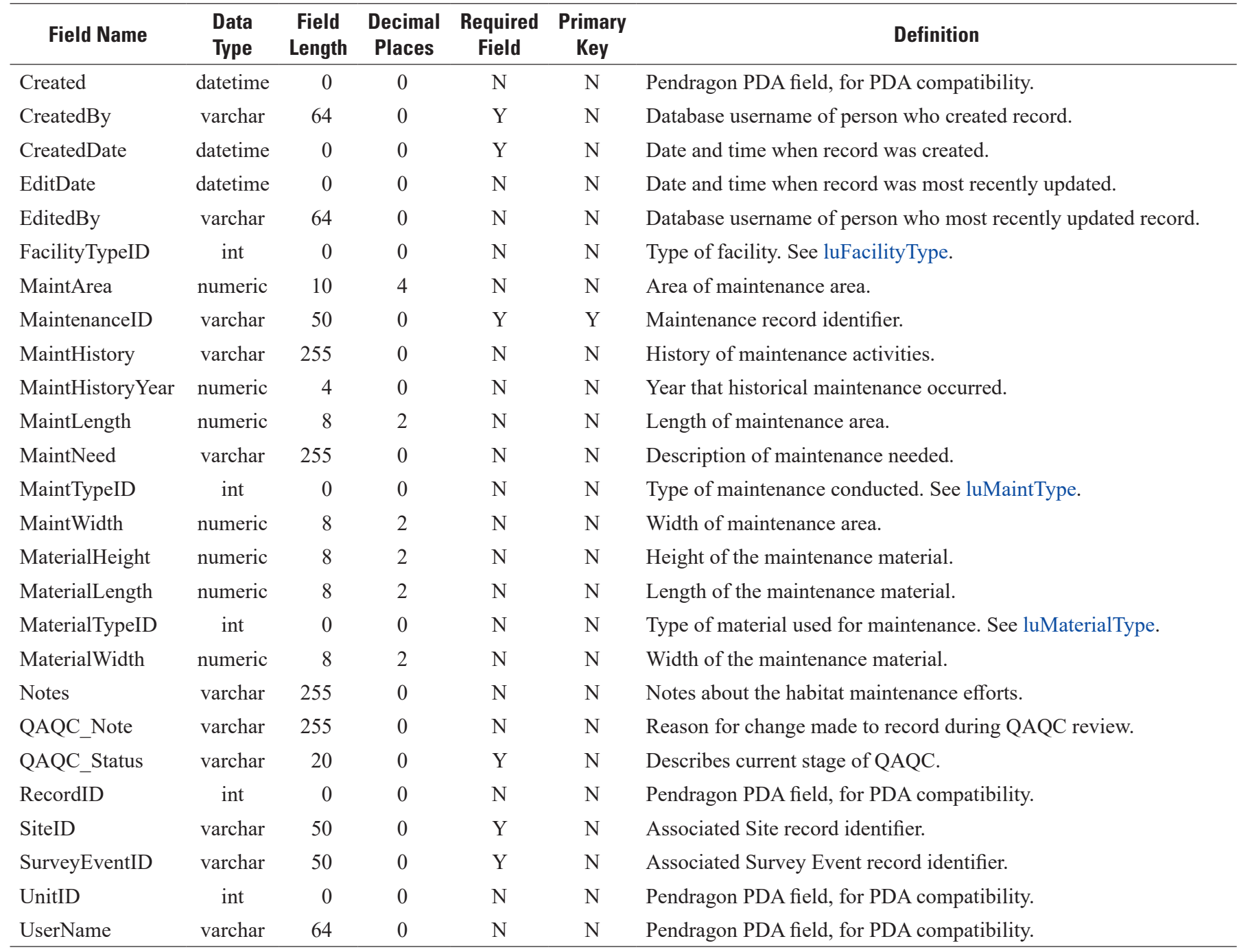


Maintenance_Affiliate Table

\begin{tabular}{lcccccl}
\hline \multicolumn{1}{c}{ Field Name } & $\begin{array}{c}\text { Data } \\
\text { Type }\end{array}$ & $\begin{array}{c}\text { Field } \\
\text { Length }\end{array}$ & $\begin{array}{c}\text { Decimal } \\
\text { Places }\end{array}$ & $\begin{array}{c}\text { Required } \\
\text { Field }\end{array}$ & $\begin{array}{c}\text { Primary } \\
\text { Key }\end{array}$ & Definition \\
\hline AffiliateID & int & 0 & 0 & Y & N & Person or entity who conducted the maintenance. \\
Created & datetime & 0 & 0 & N & N & Pendragon PDA field, for PDA compatibility. \\
CreatedBy & varchar & 64 & 0 & Y & N & Database username of person who created record. \\
CreatedDate & datetime & 0 & 0 & Y & N & Date and time when record was created. \\
EditDate & datetime & 0 & 0 & N & N & Date and time when record was most recently updated. \\
EditedBy & varchar & 64 & 0 & N & N & Database username of person who most recently updated record. \\
Maintenance_ & bigint & 19 & 0 & Y & Y & Maintenance_Affiliate record identifier. \\
$\quad$ AffiliateID & & & & & & \\
MaintenanceID & varchar & 50 & 0 & Y & N & Associated Maintenance record identifier. \\
MaintenanceRole & varchar & 25 & 0 & $\mathrm{~N}$ & $\mathrm{~N}$ & Role of affiliate involved in the maintenance activity. \\
QAQC_Note & varchar & 255 & 0 & $\mathrm{~N}$ & $\mathrm{~N}$ & Reason for change made to record during QAQC review. \\
QAQC_Status & varchar & 20 & 0 & $\mathrm{Y}$ & $\mathrm{N}$ & Describes current stage of QAQC. \\
RecordID & int & 0 & 0 & $\mathrm{~N}$ & $\mathrm{~N}$ & Pendragon PDA field, for PDA compatibility. \\
UnitID & int & 0 & 0 & $\mathrm{~N}$ & $\mathrm{~N}$ & Pendragon PDA field, for PDA compatibility. \\
UserName & varchar & 64 & 0 & $\mathrm{~N}$ & $\mathrm{~N}$ & Pendragon PDA field, for PDA compatibility. \\
\hline
\end{tabular}

MaintenancePoints Table

\begin{tabular}{lcccccl}
\hline \multicolumn{1}{c}{ Field Name } & $\begin{array}{c}\text { Data } \\
\text { Type }\end{array}$ & $\begin{array}{c}\text { Field } \\
\text { Length }\end{array}$ & $\begin{array}{c}\text { Decimal } \\
\text { Places }\end{array}$ & $\begin{array}{c}\text { Required } \\
\text { Field }\end{array}$ & $\begin{array}{c}\text { Primary } \\
\text { Key }\end{array}$ & Definition \\
\hline Created & datetime & 0 & 0 & $\mathrm{~N}$ & $\mathrm{~N}$ & Pendragon PDA field, for PDA compatibility. \\
CreatedBy & varchar & 64 & 0 & $\mathrm{Y}$ & $\mathrm{N}$ & Database username of person who created record. \\
CreatedDate & datetime & 0 & 0 & $\mathrm{Y}$ & $\mathrm{N}$ & Date and time when record was created. \\
EditDate & datetime & 0 & 0 & $\mathrm{~N}$ & $\mathrm{~N}$ & Date and time when record was most recently updated. \\
EditedBy & varchar & 64 & 0 & $\mathrm{~N}$ & $\mathrm{~N}$ & Database username of person who most recently updated record. \\
MaintenanceID & varchar & 50 & 0 & $\mathrm{Y}$ & $\mathrm{N}$ & Associated Maintenance record identifier. \\
MaintenancePointID & varchar & 50 & 0 & $\mathrm{Y}$ & $\mathrm{Y}$ & Maintenance Point record identifier. \\
MaintPointNum & int & 0 & 0 & $\mathrm{~N}$ & $\mathrm{~N}$ & Maintenance Point number. \\
MaintPointType & varchar & 15 & 0 & $\mathrm{~N}$ & $\mathrm{~N}$ & Type of maintenance point. See luMaintPointType. \\
PointID & varchar & 50 & 0 & $\mathrm{~N}$ & $\mathrm{~N}$ & Associated Spatial Feature record identifier. \\
QAQC_Note & varchar & 255 & 0 & $\mathrm{~N}$ & $\mathrm{~N}$ & Reason for change made to record during QAQC review. \\
QAQC_Status & varchar & 20 & 0 & $\mathrm{Y}$ & $\mathrm{N}$ & Describes current stage of QAQC. \\
RecordID & int & 0 & 0 & $\mathrm{~N}$ & $\mathrm{~N}$ & Pendragon PDA field, for PDA compatibility. \\
UnitID & int & 0 & 0 & $\mathrm{~N}$ & $\mathrm{~N}$ & Pendragon PDA field, for PDA compatibility. \\
UserName & varchar & 64 & 0 & $\mathrm{~N}$ & $\mathrm{~N}$ & Pendragon PDA field, for PDA compatibility. \\
\hline
\end{tabular}

ManagementActivities Table

\begin{tabular}{lcccccl}
\hline \multicolumn{1}{c}{ Field Name } & $\begin{array}{c}\text { Data } \\
\text { Type }\end{array}$ & $\begin{array}{c}\text { Field } \\
\text { Length }\end{array}$ & $\begin{array}{c}\text { Decimal } \\
\text { Places }\end{array}$ & $\begin{array}{c}\text { Required } \\
\text { Field }\end{array}$ & $\begin{array}{c}\text { Primary } \\
\text { Key }\end{array}$ & Definition \\
\hline ActivityTypeID & int & 0 & 0 & $\mathrm{~N}$ & $\mathrm{~N}$ & Type of management activity. See luActivityType. \\
ActualEndDate & datetime & 0 & 0 & $\mathrm{~N}$ & $\mathrm{~N}$ & True end date of management activity. \\
CreatedBy & varchar & 64 & 0 & $\mathrm{Y}$ & $\mathrm{N}$ & Database username of person who created record. \\
CreatedDate & datetime & 0 & 0 & $\mathrm{Y}$ & $\mathrm{N}$ & Date and time when record was created. \\
EditDate & datetime & 0 & 0 & $\mathrm{~N}$ & $\mathrm{~N}$ & Date and time when record was most recently updated.
\end{tabular}


ManagementActivities Table-Continued

\begin{tabular}{|c|c|c|c|c|c|c|}
\hline Field Name & $\begin{array}{l}\text { Data } \\
\text { Type }\end{array}$ & $\begin{array}{l}\text { Field } \\
\text { Length }\end{array}$ & $\begin{array}{l}\text { Decimal } \\
\text { Places }\end{array}$ & $\begin{array}{l}\text { Required } \\
\text { Field }\end{array}$ & $\begin{array}{l}\text { Primary } \\
\text { Key }\end{array}$ & Definition \\
\hline EditedBy & varchar & 64 & 0 & $\mathrm{~N}$ & $\mathrm{~N}$ & $\begin{array}{l}\text { Database username of person who most recently updated } \\
\text { record. }\end{array}$ \\
\hline Goals & varchar & 255 & 0 & $\mathrm{~N}$ & $\mathrm{~N}$ & Goals set forth for this management activity. \\
\hline ManagementActivityID & bigint & 19 & 0 & Y & Y & ManagementActivity record identifier. \\
\hline MgtPlanDetailID & bigint & 19 & 0 & Y & $\mathrm{N}$ & Associated MgtPlanDetail record identifier. \\
\hline PostactivityConditions & varchar & 255 & 0 & $\mathrm{~N}$ & $\mathrm{~N}$ & $\begin{array}{l}\text { Describe the habitat conditions after management activity } \\
\text { completed. }\end{array}$ \\
\hline PreactivityConditions & varchar & 255 & 0 & $\mathrm{~N}$ & $\mathrm{~N}$ & $\begin{array}{l}\text { Describe the habitat conditions before management activity } \\
\text { started. }\end{array}$ \\
\hline ProposedEndDate & datetime & 0 & 0 & $\mathrm{~N}$ & $\mathrm{~N}$ & Proposed end date of management activity. \\
\hline
\end{tabular}

ManagementPlans Table

\begin{tabular}{lcccccl}
\hline \multirow{2}{*}{ Field Name } & $\begin{array}{c}\text { Data } \\
\text { Type }\end{array}$ & $\begin{array}{c}\text { Field } \\
\text { Length }\end{array}$ & $\begin{array}{c}\text { Decimal } \\
\text { Places }\end{array}$ & $\begin{array}{c}\text { Required } \\
\text { Field }\end{array}$ & $\begin{array}{c}\text { Primary } \\
\text { Key }\end{array}$ & Definition \\
\hline CreatedBy & varchar & 64 & 0 & $\mathrm{Y}$ & $\mathrm{N}$ & Database username of person who created record. \\
CreatedDate & datetime & 0 & 0 & $\mathrm{Y}$ & $\mathrm{N}$ & Date and time when record was created. \\
EditDate & datetime & 0 & 0 & $\mathrm{~N}$ & $\mathrm{~N}$ & Date and time when record was most recently updated. \\
EditedBy & varchar & 64 & 0 & $\mathrm{~N}$ & $\mathrm{~N}$ & Database username of person who most recently updated record. \\
ManagementPlanID & bigint & 19 & 0 & $\mathrm{Y}$ & $\mathrm{Y}$ & ManagementPlan record identifier. \\
Manager & int & 0 & 0 & $\mathrm{~N}$ & $\mathrm{~N}$ & Affiliate ID of person who manages the plan. See Affiliate table. \\
MgtPlanDesc & varchar & 255 & 0 & $\mathrm{~N}$ & $\mathrm{~N}$ & Description of the management plan. \\
MgtPlanName & varchar & 255 & 0 & $\mathrm{Y}$ & $\mathrm{N}$ & Name of the management plan. \\
QAQC_Note & varchar & 255 & 0 & $\mathrm{~N}$ & $\mathrm{~N}$ & Reason for change made to record during QAQC review. \\
QAQC_Status & varchar & 20 & 0 & $\mathrm{Y}$ & $\mathrm{N}$ & Describes current stage of QAQC. \\
SiteID & varchar & 50 & 0 & $\mathrm{Y}$ & $\mathrm{N}$ & Associated Site record identifier. \\
\hline
\end{tabular}

MgmtActivityMonitoring Table

\begin{tabular}{lcccccc}
\hline \multicolumn{1}{c}{ Field Name } & $\begin{array}{c}\text { Data } \\
\text { Type }\end{array}$ & $\begin{array}{c}\text { Field } \\
\text { Length }\end{array}$ & $\begin{array}{c}\text { Decimal } \\
\text { Places }\end{array}$ & $\begin{array}{c}\text { Required } \\
\text { Field }\end{array}$ & $\begin{array}{c}\text { Primary } \\
\text { Key }\end{array}$ & Definition \\
\hline CreatedBy & varchar & 64 & 0 & $\mathrm{Y}$ & $\mathrm{N}$ & Database username of person who created record. \\
CreatedDate & datetime & 0 & 0 & $\mathrm{Y}$ & $\mathrm{N}$ & Date and time when record was created. \\
EditDate & datetime & 0 & 0 & $\mathrm{~N}$ & $\mathrm{~N}$ & Date and time when record was most recently updated. \\
EditedBy & varchar & 64 & 0 & $\mathrm{~N}$ & $\mathrm{~N}$ & Database username of person who most recently updated \\
& & & & & & record. \\
ManagementActivityID & bigint & 19 & 0 & $\mathrm{Y}$ & $\mathrm{N}$ & Associated ManagementActivity record identifier. \\
MgtActivityMonitoringID & bigint & 19 & 0 & $\mathrm{Y}$ & $\mathrm{Y}$ & MgtActivity Monitoring record identifier. \\
PropMonitoringTypeID & int & 0 & 0 & $\mathrm{~N}$ & $\mathrm{~N}$ & Proposed monitoring type. See luMonitoringType. \\
PropSurveyArea & numeric & 8 & 2 & $\mathrm{~N}$ & $\mathrm{~N}$ & Proposed survey area. \\
PropSurveyNum & int & 0 & 0 & $\mathrm{~N}$ & $\mathrm{~N}$ & Proposed number of surveys. \\
PropYearsMonitoring & int & 0 & 0 & $\mathrm{~N}$ & $\mathrm{~N}$ & Proposed years of monitoring.
\end{tabular}


MgmtActivityMonitoring Table-Continued

\begin{tabular}{lcccccc}
\hline \multicolumn{1}{c}{ Field Name } & $\begin{array}{c}\text { Data } \\
\text { Type }\end{array}$ & $\begin{array}{c}\text { Field } \\
\text { Length }\end{array}$ & $\begin{array}{c}\text { Decimal } \\
\text { Places }\end{array}$ & $\begin{array}{c}\text { Required } \\
\text { Field }\end{array}$ & $\begin{array}{c}\text { Primary } \\
\text { Key }\end{array}$ & Definition \\
\hline QAQC_Note & varchar & 255 & 0 & $\mathrm{~N}$ & $\mathrm{~N}$ & Reason for change made to record during QAQC review. \\
QAQC_Status & varchar & 20 & 0 & $\mathrm{Y}$ & $\mathrm{N}$ & $\begin{array}{c}\text { Describes current stage of QAQC. } \\
\text { Required }\end{array}$ \\
& bit & 0 & 0 & $\mathrm{~N}$ & $\mathrm{~N}$ & $\begin{array}{c}\text { Y/N. Is the monitoring activity required as part of the } \\
\text { management plan? }\end{array}$ \\
\hline
\end{tabular}

\section{MgtPlanDetails Table}

\begin{tabular}{|c|c|c|c|c|c|c|}
\hline Field Name & $\begin{array}{l}\text { Data } \\
\text { Type }\end{array}$ & $\begin{array}{c}\text { Field } \\
\text { Length }\end{array}$ & $\begin{array}{c}\text { Decimal } \\
\text { Places }\end{array}$ & $\begin{array}{l}\text { Required } \\
\text { Field }\end{array}$ & $\begin{array}{c}\text { Primary } \\
\text { Key }\end{array}$ & Definition \\
\hline Comments & varchar & 255 & 0 & $\mathrm{~N}$ & $\mathrm{~N}$ & Comments about the Management plan details. \\
\hline CreatedDate & datetime & 0 & 0 & $\mathrm{Y}$ & $\mathrm{N}$ & Date and time when record was created. \\
\hline Description & varchar & 255 & 0 & $\mathrm{~N}$ & $\mathrm{~N}$ & Description of the management plan. \\
\hline EndDate & datetime & 0 & 0 & $\mathrm{~N}$ & $\mathrm{~N}$ & End date of the management plan. \\
\hline ManagementPlanID & bigint & 19 & 0 & $\mathrm{Y}$ & $\mathrm{N}$ & Associated ManagementPlan record identifier. \\
\hline MgtPlanDetailID & bigint & 19 & 0 & $\mathrm{Y}$ & $\mathrm{Y}$ & MgtPlanDetail record identifier. \\
\hline MgtTypeID & int & 0 & 0 & Y & $\mathrm{N}$ & Type of management. See luMgtType. \\
\hline QAQC_Note & varchar & 255 & 0 & $\mathrm{~N}$ & $\mathrm{~N}$ & Reason for change made to record during QAQC review. \\
\hline QAQC_Status & varchar & 20 & 0 & $\mathrm{Y}$ & $\mathrm{N}$ & Describes current stage of QAQC. \\
\hline StartDate & datetime & 0 & 0 & $\mathrm{~N}$ & $\mathrm{~N}$ & Start date of the management plan. \\
\hline
\end{tabular}

\section{MgtProjectStatus Table}

\begin{tabular}{lcccccl}
\hline \multicolumn{1}{c}{ Field Name } & $\begin{array}{c}\text { Data } \\
\text { Type }\end{array}$ & $\begin{array}{c}\text { Field } \\
\text { Length }\end{array}$ & $\begin{array}{c}\text { Decimal } \\
\text { Places }\end{array}$ & $\begin{array}{c}\text { Required } \\
\text { Field }\end{array}$ & $\begin{array}{c}\text { Primary } \\
\text { Key }\end{array}$ & Definition \\
\hline CreatedBy & varchar & 64 & 0 & $\mathrm{Y}$ & $\mathrm{N}$ & Database username of person who created record. \\
CreatedDate & datetime & 0 & 0 & $\mathrm{Y}$ & $\mathrm{N}$ & Date and time when record was created. \\
CurrentMilestone & smallint & 5 & 0 & $\mathrm{~N}$ & $\mathrm{~N}$ & Current project milestone to be achieved. \\
EditDate & datetime & 0 & 0 & $\mathrm{~N}$ & $\mathrm{~N}$ & Date and time when record was most recently updated. \\
EditedBy & varchar & 64 & 0 & $\mathrm{~N}$ & $\mathrm{~N}$ & Database username of person who most recently updated record. \\
MgtPlanDetailID & bigint & 19 & 0 & $\mathrm{Y}$ & $\mathrm{N}$ & Associated MgtPlanDetail record identifier. \\
MgtProjectStatusID & bigint & 19 & 0 & $\mathrm{Y}$ & $\mathrm{Y}$ & MgtProjectStatus record identifier. \\
MilestoneDate & datetime & 0 & 0 & $\mathrm{~N}$ & $\mathrm{~N}$ & Date of current milestone. \\
MilestoneTypeID & smallint & 5 & 0 & $\mathrm{~N}$ & $\mathrm{~N}$ & Type of milestone expected for project. See luMilestoneType. \\
QAQC_Note & varchar & 255 & 0 & $\mathrm{~N}$ & $\mathrm{~N}$ & Reason for change made to record during QAQC review. \\
QAQC_Status & varchar & 20 & 0 & $\mathrm{Y}$ & $\mathrm{N}$ & Describes current stage of QAQC. \\
Status & varchar & 15 & 0 & $\mathrm{~N}$ & $\mathrm{~N}$ & Status of the management project. See luMgtProjectStatus. \\
TotalMilestones & smallint & 5 & 0 & $\mathrm{~N}$ & $\mathrm{~N}$ & Total number of milestones expected for project. \\
\hline
\end{tabular}


ModeledHabitatSuitability Table

\begin{tabular}{|c|c|c|c|c|c|c|}
\hline Field Name & $\begin{array}{l}\text { Data } \\
\text { Type }\end{array}$ & $\begin{array}{l}\text { Field } \\
\text { Length }\end{array}$ & $\begin{array}{l}\text { Decimal } \\
\text { Places }\end{array}$ & $\begin{array}{l}\text { Required } \\
\text { Field }\end{array}$ & $\begin{array}{l}\text { Primary } \\
\text { Key }\end{array}$ & Definition \\
\hline AffiliateID & int & 0 & 0 & $\mathrm{~N}$ & $\mathrm{~N}$ & Associated Affiliate record identifier. \\
\hline CreatedBy & varchar & 64 & 0 & Y & $\mathrm{N}$ & Database username of person who created record. \\
\hline CreatedDate & datetime & 0 & 0 & Y & $\mathrm{N}$ & Date and time when record was created. \\
\hline Method & varchar & 50 & 0 & $\mathrm{~N}$ & $\mathrm{~N}$ & Description of model used. \\
\hline $\begin{array}{l}\text { ModeledHabitatSuit- } \\
\text { abilityID }\end{array}$ & varchar & 50 & 0 & Y & Y & ModeledHabitatSuitability record identifier. \\
\hline ModelRank & varchar & 30 & 0 & $\mathrm{~N}$ & $\mathrm{~N}$ & Rank of the model. \\
\hline SiteID & varchar & 50 & 0 & $\mathrm{~N}$ & $\mathrm{~N}$ & Associated site record identifier. \\
\hline TaxaID & varchar & 25 & 0 & $\mathrm{~N}$ & $\mathrm{~N}$ & $\begin{array}{l}\text { Taxonomic Serial Number (www.itis.gov) of species that model } \\
\text { was created for. }\end{array}$ \\
\hline UnitID & int & 0 & 0 & $\mathrm{~N}$ & $\mathrm{~N}$ & Pendragon PDA field, for PDA compatibility. \\
\hline UserName & varchar & 64 & 0 & $\mathrm{~N}$ & $\mathrm{~N}$ & Pendragon PDA field, for PDA compatibility. \\
\hline
\end{tabular}

\section{NestCreationDetails Table}

\begin{tabular}{|c|c|c|c|c|c|c|}
\hline Field Name & $\begin{array}{l}\text { Data } \\
\text { Type }\end{array}$ & $\begin{array}{l}\text { Field } \\
\text { Length }\end{array}$ & $\begin{array}{l}\text { Decimal } \\
\text { Places }\end{array}$ & $\begin{array}{l}\text { Required } \\
\text { Field }\end{array}$ & $\begin{array}{l}\text { Primary } \\
\text { Key }\end{array}$ & Definition \\
\hline ConstructionType & varchar & 25 & 0 & $\mathrm{~N}$ & $\mathrm{~N}$ & Type of construction used to create nest. \\
\hline CreatedBy & varchar & 64 & 0 & Y & $\mathrm{N}$ & Database username of person who created record. \\
\hline CreatedDate & datetime & 0 & 0 & Y & $\mathrm{N}$ & Date and time when record was created. \\
\hline EditedBy & varchar & 64 & 0 & $\mathrm{~N}$ & $\mathrm{~N}$ & Database username of person who most recently updated record. \\
\hline Height & numeric & 8 & 2 & $\mathrm{~N}$ & $\mathrm{~N}$ & Height of the nest. \\
\hline InstallType & varchar & 25 & 0 & $\mathrm{~N}$ & $\mathrm{~N}$ & How the nest was installed. \\
\hline Length & numeric & 8 & 2 & $\mathrm{~N}$ & $\mathrm{~N}$ & Length of the nest. \\
\hline $\begin{array}{l}\text { NestCreationDetail } \\
\text { ID }\end{array}$ & bigint & 19 & 0 & Y & $\mathrm{Y}$ & NestCreationDetail record identifier. \\
\hline NestID & varchar & 50 & 0 & $\mathrm{~N}$ & $\mathrm{~N}$ & Associated Nest record identifier. \\
\hline NestName & varchar & 50 & 0 & $\mathrm{~N}$ & $\mathrm{~N}$ & Name of the nest. \\
\hline PointID & varchar & 50 & 0 & $\mathrm{~N}$ & $\mathrm{~N}$ & $\begin{array}{l}\text { Associated SpatialFeature record identifier. Referred to as PointID } \\
\text { in some tables. }\end{array}$ \\
\hline QAQC_Note & varchar & 255 & 0 & $\mathrm{~N}$ & $\mathrm{~N}$ & Reason for change made to record during QAQC review. \\
\hline QAQC_Status & varchar & 20 & 0 & $\mathrm{Y}$ & $\mathrm{N}$ & Describes current stage of QAQC. \\
\hline RecordID & int & 0 & 0 & $\mathrm{~N}$ & $\mathrm{~N}$ & Pendragon PDA field, for PDA compatibility. \\
\hline
\end{tabular}




\begin{tabular}{lcccccl}
\multicolumn{2}{c}{ NestCreationDetails Table-Continued } & & & \\
\multicolumn{1}{c}{ Field Name } & $\begin{array}{c}\text { Data } \\
\text { Type }\end{array}$ & $\begin{array}{c}\text { Field } \\
\text { Length }\end{array}$ & $\begin{array}{c}\text { Decimal } \\
\text { Places }\end{array}$ & $\begin{array}{c}\text { Required } \\
\text { Field }\end{array}$ & $\begin{array}{c}\text { Primary } \\
\text { Key }\end{array}$ & Definition \\
\hline SiteSlope & numeric & 5 & 2 & $\mathrm{~N}$ & $\mathrm{~N}$ & Slope at the site of the nest. \\
TaxaObservationID & varchar & 50 & 0 & $\mathrm{Y}$ & $\mathrm{N}$ & Associated TaxaObservation record identifier. \\
UnitID & int & 0 & 0 & $\mathrm{~N}$ & $\mathrm{~N}$ & Pendragon PDA field, for PDA compatibility. \\
UserName & varchar & 64 & 0 & $\mathrm{~N}$ & $\mathrm{~N}$ & Pendragon PDA field, for PDA compatibility. \\
Width & numeric & 8 & 2 & $\mathrm{~N}$ & $\mathrm{~N}$ & Width of the nest. \\
\hline
\end{tabular}

\section{NestEntranceDetails Table}

\begin{tabular}{lcccccl}
\hline \multicolumn{1}{c}{ Field Name } & $\begin{array}{c}\text { Data } \\
\text { Type }\end{array}$ & $\begin{array}{c}\text { Field } \\
\text { Length }\end{array}$ & $\begin{array}{c}\text { Decimal } \\
\text { Places }\end{array}$ & $\begin{array}{c}\text { Required } \\
\text { Field }\end{array}$ & $\begin{array}{c}\text { Primary } \\
\text { Key }\end{array}$ & Definition \\
\hline Created & datetime & 0 & 0 & $\mathrm{~N}$ & $\mathrm{~N}$ & Database username of person who created record. \\
CreatedBy & varchar & 64 & 0 & $\mathrm{Y}$ & $\mathrm{N}$ & Date and time when record was created. \\
CreatedDate & datetime & 0 & 0 & $\mathrm{Y}$ & $\mathrm{N}$ & Date and time when record was most recently updated. \\
EditDate & datetime & 0 & 0 & $\mathrm{~N}$ & $\mathrm{~N}$ & Database username of person who most recently updated record. \\
EditedBy & varchar & 64 & 0 & $\mathrm{~N}$ & $\mathrm{~N}$ & Database username of person who created record. \\
EntranceDirection & varchar & 3 & 0 & $\mathrm{~N}$ & $\mathrm{~N}$ & Abbreviation of direction that entrance faces. See luDirection. \\
EntranceNumber & int & 0 & 0 & $\mathrm{~N}$ & $\mathrm{~N}$ & Number of entrances. \\
NestCreationDetail & bigint & 19 & 0 & $\mathrm{Y}$ & $\mathrm{N}$ & Associated NestCreationDetail record identifier. \\
$\quad$ ID & & & & & & \\
NestEntranceDetail & bigint & 19 & 0 & $\mathrm{Y}$ & $\mathrm{Y}$ & NestEntranceDetails record identifier. \\
ID & & & & & & \\
QAQC_Note & varchar & 255 & 0 & $\mathrm{~N}$ & $\mathrm{~N}$ & Reason for change made to record during QAQC review. \\
QAQC_Status & varchar & 20 & 0 & $\mathrm{Y}$ & $\mathrm{N}$ & Describes current stage of QAQC. \\
RecordID & int & 0 & 0 & $\mathrm{~N}$ & $\mathrm{~N}$ & Pendragon PDA field, for PDA compatibility. \\
UnitID & int & 0 & 0 & $\mathrm{~N}$ & $\mathrm{~N}$ & Pendragon PDA field, for PDA compatibility. \\
UserName & varchar & 64 & 0 & $\mathrm{~N}$ & $\mathrm{~N}$ & Pendragon PDA field, for PDA compatibility. \\
\hline
\end{tabular}

\section{NestID Table}

\begin{tabular}{lcccccc}
\hline Field Name & $\begin{array}{c}\text { Data } \\
\text { Type }\end{array}$ & $\begin{array}{c}\text { Field } \\
\text { Length }\end{array}$ & $\begin{array}{c}\text { Decimal } \\
\text { Places }\end{array}$ & $\begin{array}{c}\text { Required } \\
\text { Field }\end{array}$ & $\begin{array}{c}\text { Primary } \\
\text { Key }\end{array}$ & Definition \\
\hline Created & datetime & 0 & 0 & $\mathrm{~N}$ & $\mathrm{~N}$ & Database username of person who created record. \\
CreatedBy & varchar & 64 & 0 & $\mathrm{Y}$ & $\mathrm{N}$ & Date and time when record was created. \\
CreatedDate & datetime & 0 & 0 & $\mathrm{Y}$ & $\mathrm{N}$ & Date and time when record was most recently updated. \\
EditDate & datetime & 0 & 0 & $\mathrm{~N}$ & $\mathrm{~N}$ & Database username of person who most recently updated record. \\
EditedBy & varchar & 64 & 0 & $\mathrm{~N}$ & $\mathrm{~N}$ & Database username of person who created record. \\
NestDescID & varchar & 50 & 0 & $\mathrm{Y}$ & $\mathrm{N}$ & NestDecription identifier. \\
NestID & varchar & 50 & 0 & $\mathrm{Y}$ & $\mathrm{Y}$ & NestID record identifier. \\
NestIdentifier & char & 5 & 0 & $\mathrm{~N}$ & $\mathrm{~N}$ & Unique identifier of nest. \\
NestIDNum & varchar & 50 & 0 & $\mathrm{Y}$ & $\mathrm{N}$ & NestID number. \\
Notes & varchar & 200 & 0 & $\mathrm{~N}$ & $\mathrm{~N}$ & Notes about the nest. \\
RecordID & int & 0 & 0 & $\mathrm{~N}$ & $\mathrm{~N}$ & Pendragon PDA field, for PDA compatibility. \\
UnitID & int & 0 & 0 & $\mathrm{~N}$ & $\mathrm{~N}$ & Pendragon PDA field, for PDA compatibility. \\
UserName & varchar & 64 & 0 & $\mathrm{~N}$ & $\mathrm{~N}$ & Pendragon PDA field, for PDA compatibility. \\
\hline
\end{tabular}


NestMorph Table

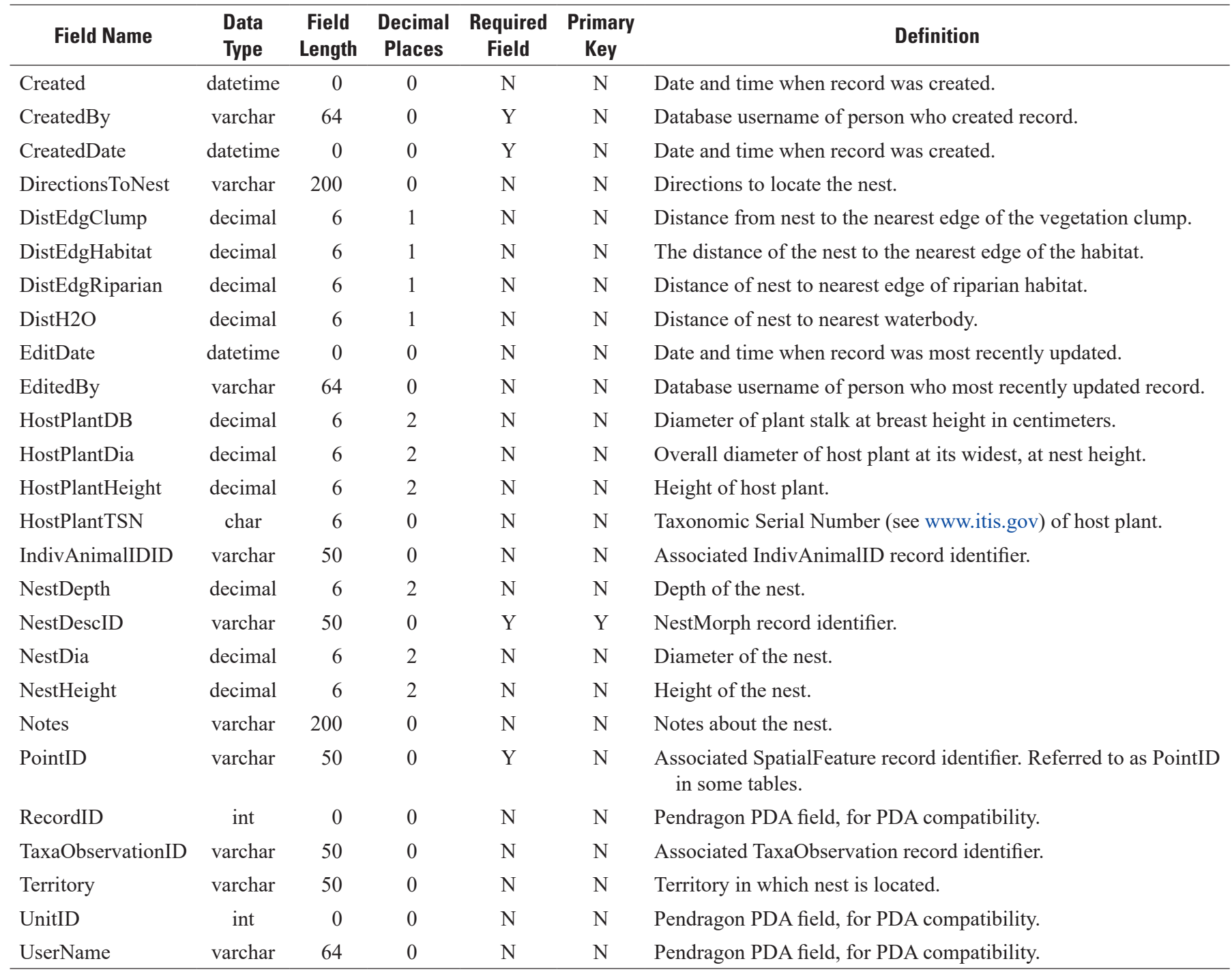

\section{ObservationDetectionMethods Table}

\begin{tabular}{|c|c|c|c|c|c|c|}
\hline Field Name & $\begin{array}{l}\text { Data } \\
\text { Type }\end{array}$ & $\begin{array}{l}\text { Field } \\
\text { Length }\end{array}$ & $\begin{array}{l}\text { Decimal } \\
\text { Places }\end{array}$ & $\begin{array}{l}\text { Required } \\
\text { Field }\end{array}$ & $\begin{array}{c}\text { Primary } \\
\text { Key }\end{array}$ & Definition \\
\hline Created & datetime & 0 & 0 & $\mathrm{~N}$ & $\mathrm{~N}$ & Pendragon PDA field, for PDA compatibility. \\
\hline CreatedBy & varchar & 64 & 0 & Y & $\mathrm{N}$ & Database username of person who created record. \\
\hline CreatedDate & datetime & 0 & 0 & Y & $\mathrm{N}$ & Date and time when record was created. \\
\hline DetectionMethodID & int & 0 & 0 & Y & $\mathrm{N}$ & Method used to detect species. See luDetectionMethod. \\
\hline $\begin{array}{l}\text { DetectopMethod- } \\
\text { Notes }\end{array}$ & varchar & 200 & 0 & $\mathrm{~N}$ & $\mathrm{~N}$ & Notes about the method used to detect the species. \\
\hline EditDate & datetime & 0 & 0 & $\mathrm{~N}$ & $\mathrm{~N}$ & Date and time when record was most recently updated. \\
\hline EditedBy & varchar & 64 & 0 & $\mathrm{~N}$ & $\mathrm{~N}$ & Database username of person who most recently updated record. \\
\hline $\begin{array}{l}\text { ObservationDetec- } \\
\text { tionMethodsID }\end{array}$ & varchar & 50 & 0 & Y & $\mathrm{Y}$ & ObservationDetectionMethods record identifier. \\
\hline
\end{tabular}




\begin{tabular}{|c|c|c|c|c|c|c|}
\hline Field Name & $\begin{array}{l}\text { Data } \\
\text { Type }\end{array}$ & $\begin{array}{c}\text { Field } \\
\text { Length }\end{array}$ & $\begin{array}{l}\text { Decimal } \\
\text { Places }\end{array}$ & $\begin{array}{l}\text { Required } \\
\text { Field }\end{array}$ & $\begin{array}{c}\text { Primary } \\
\text { Key }\end{array}$ & Definition \\
\hline $\begin{array}{l}\text { TaxaObservation- } \\
\text { DetailsID }\end{array}$ & varchar & 50 & 0 & $\mathrm{~N}$ & $\mathrm{~N}$ & Associated TaxaObservationDetails record identifier. \\
\hline UnitID & int & 0 & 0 & $\mathrm{~N}$ & $\mathrm{~N}$ & Pendragon PDA field, for PDA compatibility. \\
\hline UserName & varchar & 64 & 0 & $\mathrm{~N}$ & $\mathrm{~N}$ & Pendragon PDA field, for PDA compatibility. \\
\hline
\end{tabular}

\section{ObservationVerificationTable}

\begin{tabular}{lcccccl}
\hline \multicolumn{1}{c}{ Field Name } & $\begin{array}{c}\text { Data } \\
\text { Type }\end{array}$ & $\begin{array}{c}\text { Field } \\
\text { Length }\end{array}$ & $\begin{array}{c}\text { Decimal } \\
\text { Places }\end{array}$ & $\begin{array}{c}\text { Required } \\
\text { Field }\end{array}$ & $\begin{array}{c}\text { Primary } \\
\text { Key }\end{array}$ & Definition \\
\hline AffiliateID & int & 0 & 0 & $\mathrm{Y}$ & $\mathrm{N}$ & AffiliateID of person who verified the species identification. \\
IsVerified & char & 1 & 0 & $\mathrm{~N}$ & $\mathrm{~N}$ & Is the species observation verified? Y/N \\
Notes & varchar & 255 & 0 & $\mathrm{~N}$ & $\mathrm{~N}$ & Notes about the species observation verification. \\
ObVerificationID & varchar & 50 & 0 & $\mathrm{Y}$ & $\mathrm{Y}$ & ObservationVerification record identifier. \\
TaxaObservationID & varchar & 50 & 0 & $\mathrm{Y}$ & $\mathrm{N}$ & Associated TaxaObservation record identifier. \\
VerificationMethod & varchar & 50 & 0 & $\mathrm{~N}$ & $\mathrm{~N}$ & Method used to verify species observation. See luVerifMethods. \\
\hline
\end{tabular}

OccupancyEstimates Table

\begin{tabular}{|c|c|c|c|c|c|c|}
\hline Field Name & $\begin{array}{l}\text { Data } \\
\text { Type }\end{array}$ & $\begin{array}{l}\text { Field } \\
\text { Length }\end{array}$ & $\begin{array}{l}\text { Decimal } \\
\text { Places }\end{array}$ & $\begin{array}{l}\text { Required } \\
\text { Field }\end{array}$ & $\begin{array}{l}\text { Primary } \\
\text { Key }\end{array}$ & Definition \\
\hline CreatedBy & varchar & 64 & 0 & $\mathrm{Y}$ & $\mathrm{N}$ & Database username of person who created record. \\
\hline Detectibility & decimal & 6 & 5 & $\mathrm{~N}$ & $\mathrm{~N}$ & $\begin{array}{l}\text { Estimate of the probability of detecting a species that is present during } \\
\text { a survey. }\end{array}$ \\
\hline EditDate & datetime & 0 & 0 & $\mathrm{~N}$ & $\mathrm{~N}$ & Date and time when record was most recently updated. \\
\hline ESID & varchar & 50 & 0 & $\mathrm{Y}$ & $\mathrm{N}$ & EstimateSeries record identifier. \\
\hline Notes & varchar & 250 & 0 & $\mathrm{~N}$ & $\mathrm{~N}$ & Notes about the occupancy estimate. \\
\hline NumberUnits & int & 0 & 0 & $\mathrm{~N}$ & $\mathrm{~N}$ & $\begin{array}{l}\text { Total number of units for which detected/not detected data were } \\
\text { recorded. For example, if occupancy of grid cells is estimated, how } \\
\text { many grid cells were sampled? }\end{array}$ \\
\hline QAQC_Note & varchar & 255 & 0 & $\mathrm{~N}$ & $\mathrm{~N}$ & Reason for change made to record during QAQC review. \\
\hline $\begin{array}{l}\mathrm{QAQC}_{-} \\
\text {Status }\end{array}$ & varchar & 20 & 0 & $\mathrm{Y}$ & $\mathrm{N}$ & Describes current stage of QAQC. \\
\hline Se_detect & decimal & 6 & 4 & $\mathrm{~N}$ & $\mathrm{~N}$ & Standard error of the estimate of detectability. \\
\hline Se_psi & decimal & 6 & 5 & $\mathrm{~N}$ & $\mathrm{~N}$ & Standard error of the estimate of the number of units occupied. \\
\hline Season & varchar & 15 & 0 & $\mathrm{~N}$ & $\mathrm{~N}$ & Season for which estimate was calculated. \\
\hline Version & varchar & 25 & 0 & $\mathrm{~N}$ & $\mathrm{~N}$ & Text or numbers indicating the version of the software. \\
\hline Year & varchar & 25 & 0 & $\mathrm{~N}$ & $\mathrm{~N}$ & The year(s) to which this estimate applies. \\
\hline
\end{tabular}


Person_Org Table

\begin{tabular}{|c|c|c|c|c|c|c|}
\hline Field Name & $\begin{array}{l}\text { Data } \\
\text { Type }\end{array}$ & $\begin{array}{l}\text { Field } \\
\text { Length }\end{array}$ & $\begin{array}{l}\text { Decimal } \\
\text { Places }\end{array}$ & $\begin{array}{l}\text { Required } \\
\text { Field }\end{array}$ & $\begin{array}{l}\text { Primary } \\
\text { Key }\end{array}$ & Definition \\
\hline CreatedBy & varchar & 64 & 0 & $\mathrm{Y}$ & $\mathrm{N}$ & Database username of person who created record. \\
\hline EditDate & datetime & 0 & 0 & $\mathrm{~N}$ & $\mathrm{~N}$ & Date and time when record was most recently updated. \\
\hline EditedBy & varchar & 64 & 0 & $\mathrm{~N}$ & $\mathrm{~N}$ & Database username of person who most recently updated record. \\
\hline EffectiveStartDate & datetime & 0 & 0 & $\mathrm{~N}$ & $\mathrm{~N}$ & $\begin{array}{l}\text { The beginning date of the relationship between the person and the } \\
\text { organization. }\end{array}$ \\
\hline Notes & varchar & 200 & 0 & $\mathrm{~N}$ & $\mathrm{~N}$ & Notes. \\
\hline OrganizationID & int & 0 & 0 & $\mathrm{Y}$ & $\mathrm{N}$ & $\begin{array}{l}\text { Associated Affiliate record identifier referencing the organization in } \\
\text { the relationship. }\end{array}$ \\
\hline
\end{tabular}

\section{Photolnfo Table}

\begin{tabular}{|c|c|c|c|c|c|c|}
\hline Field Name & $\begin{array}{l}\text { Data } \\
\text { Type }\end{array}$ & $\begin{array}{l}\text { Field } \\
\text { Length }\end{array}$ & $\begin{array}{l}\text { Decimal } \\
\text { Places }\end{array}$ & $\begin{array}{l}\text { Required } \\
\text { Field }\end{array}$ & $\begin{array}{l}\text { Primary } \\
\text { Key }\end{array}$ & Definition \\
\hline Created & datetime & 0 & 0 & $\mathrm{Y}$ & Y & Pendragon PDA field, for PDA compatibility. \\
\hline CreatedDate & datetime & 0 & 0 & $\mathrm{~N}$ & Y & Date and time when record was created. \\
\hline DirectionOfCamera & decimal & 4 & 1 & $\mathrm{~N}$ & $\mathrm{~N}$ & $\begin{array}{l}\text { Direction toward which camera is pointing, using } 0 \text { to } 360 \\
\text { degrees, where } 0 \text { is north. }\end{array}$ \\
\hline EditedBy & varchar & 64 & 0 & $\mathrm{~N}$ & $\mathrm{~N}$ & Database username of person who most recently updated record. \\
\hline FrameNumber & varchar & 25 & 0 & $\mathrm{~N}$ & $\mathrm{~N}$ & Frame number of photo, taken from camera. \\
\hline MediaLabel & varchar & 25 & 0 & $\mathrm{~N}$ & $\mathrm{~N}$ & Identifier used on media. \\
\hline NumPhotos & int & 0 & 0 & $\mathrm{~N}$ & $\mathrm{~N}$ & Number of photos taken of a single subject. \\
\hline PhotoLocation & varchar & 200 & 0 & $\mathrm{~N}$ & $\mathrm{~N}$ & Physical location of photo (machine: directory). \\
\hline PhotoName & varchar & 50 & 0 & $\mathrm{~N}$ & $\mathrm{~N}$ & Name of photo/photo file. \\
\hline PhotoNotes & varchar & 200 & 0 & $\mathrm{~N}$ & $\mathrm{~N}$ & Notes regarding this photo. \\
\hline PhotoQualityID & int & 0 & 0 & $\mathrm{~N}$ & $\mathrm{~N}$ & Description of photo's quality. See luPhotoQuality table. \\
\hline PhotoTypeID & int & 0 & 0 & $\mathrm{~N}$ & $\mathrm{~N}$ & $\begin{array}{l}\text { Description of physical storage media of photo. See luPhotoType } \\
\text { table. }\end{array}$ \\
\hline RecordID & int & 0 & 0 & $\mathrm{~N}$ & $\mathrm{~N}$ & Pendragon PDA field, for PDA compatibility. \\
\hline UnitID & int & 0 & 0 & $\mathrm{~N}$ & $\mathrm{~N}$ & Pendragon PDA field, for PDA compatibility. \\
\hline UserName & varchar & 64 & 0 & $\mathrm{~N}$ & $\mathrm{~N}$ & Pendragon PDA field, for PDA compatibility. \\
\hline
\end{tabular}


Photolnfo_Actions Table

\begin{tabular}{lcccccl}
\hline Field Name & $\begin{array}{c}\text { Data } \\
\text { Type }\end{array}$ & $\begin{array}{c}\text { Field } \\
\text { Length }\end{array}$ & $\begin{array}{c}\text { Decimal } \\
\text { Places }\end{array}$ & $\begin{array}{c}\text { Required } \\
\text { Field }\end{array}$ & $\begin{array}{c}\text { Primary } \\
\text { Key }\end{array}$ & Definition \\
\hline ActionID & bigint & 19 & 0 & $\mathrm{Y}$ & $\mathrm{Y}$ & Associated Actions record identifer. \\
CreatedBy & varchar & 64 & 0 & $\mathrm{Y}$ & $\mathrm{N}$ & Database username of person who created record. \\
CreatedDate & datetime & 0 & 0 & $\mathrm{Y}$ & $\mathrm{N}$ & Date and time when record was created. \\
EditDate & datetime & 0 & 0 & $\mathrm{~N}$ & $\mathrm{~N}$ & Date and time when record was most recently updated. \\
EditedBy & varchar & 64 & 0 & $\mathrm{~N}$ & $\mathrm{~N}$ & Database username of person who most recently updated record. \\
PhotoID & varchar & 50 & 0 & $\mathrm{Y}$ & $\mathrm{Y}$ & Associated PhotoInfo record identifier. \\
QAQC_Note & varchar & 255 & 0 & $\mathrm{~N}$ & $\mathrm{~N}$ & Reason for change made to record during QAQC review. \\
QAQC_Status & varchar & 20 & 0 & $\mathrm{Y}$ & $\mathrm{N}$ & Describes current stage of QAQC. \\
\hline
\end{tabular}

\section{PhotoInfo_HabitatObservations Table}

\begin{tabular}{lcccccl}
\hline \multicolumn{1}{c}{ Field Name } & $\begin{array}{c}\text { Data } \\
\text { Type }\end{array}$ & $\begin{array}{c}\text { Field } \\
\text { Length }\end{array}$ & $\begin{array}{c}\text { Decimal } \\
\text { Places }\end{array}$ & $\begin{array}{c}\text { Required } \\
\text { Field }\end{array}$ & $\begin{array}{c}\text { Primary } \\
\text { Key }\end{array}$ & Definition \\
\hline Created & datetime & 0 & 0 & $\mathrm{~N}$ & $\mathrm{~N}$ & Pendragon PDA field, for PDA compatibility. \\
CreatedBy & varchar & 64 & 0 & $\mathrm{Y}$ & $\mathrm{N}$ & Database username of person who created record. \\
CreatedDate & datetime & 0 & 0 & $\mathrm{Y}$ & $\mathrm{N}$ & Date and time when record was created. \\
EditDate & datetime & 0 & 0 & $\mathrm{~N}$ & $\mathrm{~N}$ & Date and time when record was most recently updated. \\
EditedBy & varchar & 64 & 0 & $\mathrm{~N}$ & $\mathrm{~N}$ & $\begin{array}{c}\text { Database username of person who most recently updated } \\
\text { HabitatObservationID }\end{array}$ \\
\cline { 2 - 5 } & varchar & 50 & 0 & $\mathrm{Y}$ & $\mathrm{Y}$ & Associated HabitatObservation record identifier. \\
PhotoID & varchar & 50 & 0 & $\mathrm{Y}$ & $\mathrm{Y}$ & Associated PhotoInfo record identifier. \\
RecordID & int & 0 & 0 & $\mathrm{~N}$ & $\mathrm{~N}$ & Pendragon PDA field, for PDA compatibility. \\
UnitID & int & 0 & 0 & $\mathrm{~N}$ & $\mathrm{~N}$ & Pendragon PDA field, for PDA compatibility. \\
UserName & varchar & 64 & 0 & $\mathrm{~N}$ & $\mathrm{~N}$ & Pendragon PDA field, for PDA compatibility. \\
\hline
\end{tabular}

PhotoInfo_IndivAnimals Table

\begin{tabular}{lcccccl}
\hline Field Name & $\begin{array}{c}\text { Data } \\
\text { Type }\end{array}$ & $\begin{array}{c}\text { Field } \\
\text { Length }\end{array}$ & $\begin{array}{c}\text { Decimal } \\
\text { Places }\end{array}$ & $\begin{array}{c}\text { Required } \\
\text { Field }\end{array}$ & $\begin{array}{c}\text { Primary } \\
\text { Key }\end{array}$ & Definition \\
\hline Created & datetime & 0 & 0 & $\mathrm{~N}$ & $\mathrm{~N}$ & Pendragon PDA field, for PDA compatibility. \\
CreatedBy & varchar & 64 & 0 & $\mathrm{Y}$ & $\mathrm{N}$ & Database username of person who created record. \\
CreatedDate & datetime & 0 & 0 & $\mathrm{Y}$ & $\mathrm{N}$ & Date and time when record was created. \\
EditDate & datetime & 0 & 0 & $\mathrm{~N}$ & $\mathrm{~N}$ & Date and time when record was most recently updated. \\
EditedBy & varchar & 64 & 0 & $\mathrm{~N}$ & $\mathrm{~N}$ & Database username of person who most recently updated record. \\
IAID & varchar & 50 & 0 & $\mathrm{Y}$ & $\mathrm{Y}$ & Associated IndivAnimals record identifier. \\
PhotoID & varchar & 50 & 0 & $\mathrm{Y}$ & $\mathrm{Y}$ & Associated PhotoInfo record identifier. \\
RecordID & int & 0 & 0 & $\mathrm{~N}$ & $\mathrm{~N}$ & Pendragon PDA field, for PDA compatibility. \\
UnitID & int & 0 & 0 & $\mathrm{~N}$ & $\mathrm{~N}$ & Pendragon PDA field, for PDA compatibility. \\
UserName & varchar & 64 & 0 & $\mathrm{~N}$ & $\mathrm{~N}$ & Pendragon PDA field, for PDA compatibility. \\
\hline
\end{tabular}


Photolnfo_MgtActivities Table

\begin{tabular}{lcccccl}
\hline \multicolumn{1}{c}{ Field Name } & $\begin{array}{c}\text { Data } \\
\text { Type }\end{array}$ & $\begin{array}{c}\text { Field } \\
\text { Length }\end{array}$ & $\begin{array}{c}\text { Decimal } \\
\text { Places }\end{array}$ & $\begin{array}{c}\text { Required } \\
\text { Field }\end{array}$ & $\begin{array}{c}\text { Primary } \\
\text { Key }\end{array}$ & Definition \\
\hline CreatedBy & varchar & 64 & 0 & $\mathrm{Y}$ & $\mathrm{N}$ & Database username of person who created record. \\
CreatedDate & datetime & 0 & 0 & $\mathrm{Y}$ & $\mathrm{N}$ & Date and time when record was created. \\
EditDate & datetime & 0 & 0 & $\mathrm{~N}$ & $\mathrm{~N}$ & Date and time when record was most recently updated. \\
EditedBy & varchar & 64 & 0 & $\mathrm{~N}$ & $\mathrm{~N}$ & Database username of person who most recently updated record. \\
Management- & bigint & 19 & 0 & $\mathrm{Y}$ & $\mathrm{Y}$ & Associated MgtActivities record identifier. \\
$\quad$ ActivityID & & & & & & \\
PhotoID & varchar & 50 & 0 & $\mathrm{Y}$ & $\mathrm{Y}$ & PhotoInfo record identifier. \\
QAQC_Note & varchar & 255 & 0 & $\mathrm{~N}$ & $\mathrm{~N}$ & Reason for change made to record during QAQC review. \\
QAQC_Status & varchar & 20 & 0 & $\mathrm{Y}$ & $\mathrm{N}$ & Describes current stage of QAQC. \\
\hline
\end{tabular}

PhotoInfo_SurveyEventSite Table

\begin{tabular}{lcccccl}
\hline \multicolumn{1}{c}{ Field Name } & $\begin{array}{c}\text { Data } \\
\text { Type }\end{array}$ & $\begin{array}{c}\text { Field } \\
\text { Length }\end{array}$ & $\begin{array}{c}\text { Decimal } \\
\text { Places }\end{array}$ & $\begin{array}{c}\text { Required } \\
\text { Field }\end{array}$ & $\begin{array}{c}\text { Primary } \\
\text { Key }\end{array}$ & Definition \\
\hline Created & datetime & 0 & 0 & $\mathrm{~N}$ & $\mathrm{~N}$ & Pendragon PDA field, for PDA compatibility. \\
CreatedBy & varchar & 64 & 0 & $\mathrm{Y}$ & $\mathrm{N}$ & Database username of person who created record. \\
CreatedDate & datetime & 0 & 0 & $\mathrm{Y}$ & $\mathrm{N}$ & Date and time when record was created. \\
EditDate & datetime & 0 & 0 & $\mathrm{~N}$ & $\mathrm{~N}$ & Date and time when record was most recently updated. \\
EditedBy & varchar & 64 & 0 & $\mathrm{~N}$ & $\mathrm{~N}$ & Database username of person who most recently updated record. \\
PhotoID & varchar & 50 & 0 & $\mathrm{Y}$ & $\mathrm{Y}$ & Associated PhotoInfo record identifier. \\
RecordID & int & 0 & 0 & $\mathrm{~N}$ & $\mathrm{~N}$ & Pendragon PDA field, for PDA compatibility. \\
SiteID & varchar & 50 & 0 & $\mathrm{Y}$ & $\mathrm{Y}$ & Associated Site record identifier. \\
SurveyEventID & varchar & 50 & 0 & $\mathrm{Y}$ & $\mathrm{Y}$ & Associated SurveyEvent record identifier. \\
UnitID & int & 0 & 0 & $\mathrm{~N}$ & $\mathrm{~N}$ & Pendragon PDA field, for PDA compatibility. \\
UserName & varchar & 64 & 0 & $\mathrm{~N}$ & $\mathrm{~N}$ & Pendragon PDA field, for PDA compatibility. \\
\hline
\end{tabular}

PhotoInfo_TaxaObDetails Table

\begin{tabular}{lcccccc}
\hline \multicolumn{1}{c}{ Field Name } & $\begin{array}{c}\text { Data } \\
\text { Type }\end{array}$ & $\begin{array}{c}\text { Field } \\
\text { Length }\end{array}$ & $\begin{array}{c}\text { Decimal } \\
\text { Places }\end{array}$ & $\begin{array}{c}\text { Required } \\
\text { Field }\end{array}$ & $\begin{array}{c}\text { Primary } \\
\text { Key }\end{array}$ & Definition \\
\hline Created & datetime & 0 & 0 & $\mathrm{~N}$ & $\mathrm{~N}$ & Pendragon PDA field, for PDA compatibility. \\
CreatedBy & varchar & 64 & 0 & $\mathrm{Y}$ & $\mathrm{N}$ & Database username of person who created record. \\
CreatedDate & datetime & 0 & 0 & $\mathrm{Y}$ & $\mathrm{N}$ & Date and time when record was created. \\
EditDate & datetime & 0 & 0 & $\mathrm{~N}$ & $\mathrm{~N}$ & Date and time when record was most recently updated. \\
EditedBy & varchar & 64 & 0 & $\mathrm{~N}$ & $\mathrm{~N}$ & $\begin{array}{c}\text { Database username of person who most recently updated } \\
\text { PhotoID }\end{array}$ \\
varchar & 50 & 0 & $\mathrm{Y}$ & $\mathrm{Y}$ & $\begin{array}{c}\text { Associated Photolnfo record identifier. } \\
\text { RecordID }\end{array}$ \\
TaxaObservationDetailsID & varchar & 50 & 0 & $\mathrm{Y}$ & $\mathrm{Y}$ & Associated TaxaObservationDetails record identifier. \\
UnitID & int & 0 & 0 & $\mathrm{~N}$ & $\mathrm{~N}$ & Pendragon PDA field, for PDA compatibility. \\
UserName & varchar & 64 & 0 & $\mathrm{~N}$ & $\mathrm{~N}$ & Pendragon PDA field, for PDA compatibility. \\
\hline
\end{tabular}


PlantEnhancementDetails Table

\begin{tabular}{|c|c|c|c|c|c|c|}
\hline Field Name & $\begin{array}{l}\text { Data } \\
\text { Type }\end{array}$ & $\begin{array}{l}\text { Field } \\
\text { Length }\end{array}$ & $\begin{array}{l}\text { Decimal } \\
\text { Places }\end{array}$ & $\begin{array}{l}\text { Required } \\
\text { Field }\end{array}$ & $\begin{array}{l}\text { Primary } \\
\text { Key }\end{array}$ & Definition \\
\hline Created & datetime & 0 & 0 & $\mathrm{~N}$ & $\mathrm{~N}$ & Pendragon PDA field, for PDA compatibility. \\
\hline CreatedDate & datetime & 0 & 0 & $\mathrm{Y}$ & $\mathrm{N}$ & Date and time when record was created. \\
\hline EditDate & datetime & 0 & 0 & $\mathrm{~N}$ & $\mathrm{~N}$ & Date and time when record was most recently updated. \\
\hline $\begin{array}{l}\text { IrrigationYears } \\
\quad \text { Planned }\end{array}$ & numeric & 5 & 2 & $\mathrm{~N}$ & $\mathrm{~N}$ & Number of years that irrigation is planned. \\
\hline Notes & varchar & 255 & 0 & $\mathrm{~N}$ & $\mathrm{~N}$ & Notes about the plant enhancement project. \\
\hline PatchDensity & numeric & 16 & 2 & $\mathrm{~N}$ & $\mathrm{~N}$ & Density of the patch. \\
\hline PlantingArea & numeric & 16 & 5 & $\mathrm{~N}$ & $\mathrm{~N}$ & Area of designated spot for plant enhancement activities. \\
\hline PlantingDensity & numeric & 16 & 2 & $\mathrm{~N}$ & $\mathrm{~N}$ & Spacing of plantings. \\
\hline PlantingFormID & int & 0 & 0 & $\mathrm{~N}$ & $\mathrm{~N}$ & Form of the plant being planted. See luPlantingForms. \\
\hline PointID & varchar & 50 & 0 & $\mathrm{~N}$ & $\mathrm{~N}$ & $\begin{array}{l}\text { Associated SpatialFeature record identifier. Referred to as PointID } \\
\text { in some tables. }\end{array}$ \\
\hline QAQC_Note & varchar & 255 & 0 & $\mathrm{~N}$ & $\mathrm{~N}$ & Reason for change made to record during QAQC review. \\
\hline QAQC_Status & varchar & 20 & 0 & $\mathrm{Y}$ & $\mathrm{N}$ & Describes current stage of QAQC. \\
\hline RecordID & int & 0 & 0 & $\mathrm{~N}$ & $\mathrm{~N}$ & Pendragon PDA field, for PDA compatibility. \\
\hline
\end{tabular}

\section{PlantHierarchy Table}

\begin{tabular}{lcccccl}
\hline \multicolumn{1}{c}{ Field Name } & $\begin{array}{c}\text { Data } \\
\text { Type }\end{array}$ & $\begin{array}{c}\text { Field } \\
\text { Length }\end{array}$ & $\begin{array}{c}\text { Decimal } \\
\text { Places }\end{array}$ & $\begin{array}{c}\text { Required } \\
\text { Field }\end{array}$ & $\begin{array}{c}\text { Primary } \\
\text { Key }\end{array}$ & Definition \\
\hline BasalPerimeter & int & 0 & 0 & $\mathrm{~N}$ & $\mathrm{~N}$ & Perimeter of the plant at its base. \\
Created & datetime & 0 & 0 & $\mathrm{~N}$ & $\mathrm{~N}$ & Pendragon PDA field, for PDA compatibility. \\
CreatedBy & varchar & 64 & 0 & $\mathrm{Y}$ & $\mathrm{N}$ & Database username of person who created record. \\
CreatedDate & datetime & 0 & 0 & $\mathrm{Y}$ & $\mathrm{N}$ & Date and time when record was created. \\
EditDate & datetime & 0 & 0 & $\mathrm{~N}$ & $\mathrm{~N}$ & Date and time when record was most recently updated. \\
EditedBy & varchar & 64 & 0 & $\mathrm{~N}$ & $\mathrm{~N}$ & Database username of person who most recently updated record. \\
HeightClass & varchar & 15 & 0 & $\mathrm{~N}$ & $\mathrm{~N}$ & Height class of the plant. See luHeightClass. \\
Heirarchy & char & 2 & 0 & $\mathrm{~N}$ & $\mathrm{~N}$ & Hierarchy of plant observation. \\
Notes & varchar & 200 & 0 & $\mathrm{~N}$ & $\mathrm{~N}$ & Notes about the plant hierarchy. \\
PercentCovered & varchar & 15 & 0 & $\mathrm{~N}$ & $\mathrm{~N}$ & Percent cover of the plant within the site/habitat. \\
Phenology & char & 6 & 0 & $\mathrm{~N}$ & $\mathrm{~N}$ & Phenology of the plant. \\
PHierarchyID & varchar & 50 & 0 & $\mathrm{Y}$ & $\mathrm{Y}$ & PlantHierarchy record identifier. \\
PODID & varchar & 50 & 0 & $\mathrm{~N}$ & $\mathrm{~N}$ & Associated PlantObservationDetails record identifier. \\
QAQC_Note & varchar & 255 & 0 & $\mathrm{~N}$ & $\mathrm{~N}$ & Reason for change made to record during QAQC review. \\
QAQC_Status & varchar & 20 & 0 & $\mathrm{Y}$ & $\mathrm{N}$ & Describes current stage of QAQC.
\end{tabular}


PlantHierarchy Table-Continued

\begin{tabular}{lcccccl}
\hline \multicolumn{1}{c}{ Field Name } & $\begin{array}{c}\text { Data } \\
\text { Type }\end{array}$ & $\begin{array}{c}\text { Field } \\
\text { Length }\end{array}$ & $\begin{array}{c}\text { Decimal } \\
\text { Places }\end{array}$ & $\begin{array}{c}\text { Required } \\
\text { Field }\end{array}$ & $\begin{array}{c}\text { Primary } \\
\text { Key }\end{array}$ & Definition \\
\hline RecordID & int & 0 & 0 & $\mathrm{~N}$ & $\mathrm{~N}$ & Pendragon PDA field, for PDA compatibility. \\
$\begin{array}{l}\text { TaxaObservation } \\
\text { DetailsID }\end{array}$ & varchar & 50 & 0 & $\mathrm{~N}$ & $\mathrm{~N}$ & Associated TaxaObservationDetails record identifier. \\
$\begin{array}{l}\text { TaxaObservationID } \\
\text { UnitID }\end{array}$ & varchar & 50 & 0 & $\mathrm{~N}$ & $\mathrm{~N}$ & Associated TaxaObservation record identifier. \\
UserName & int & 0 & 0 & $\mathrm{~N}$ & $\mathrm{~N}$ & Pendragon PDA field, for PDA compatibility. \\
& varchar & 64 & 0 & $\mathrm{~N}$ & $\mathrm{~N}$ & Pendragon PDA field, for PDA compatibility. \\
\hline
\end{tabular}

\section{PlantObservationDetails Table}

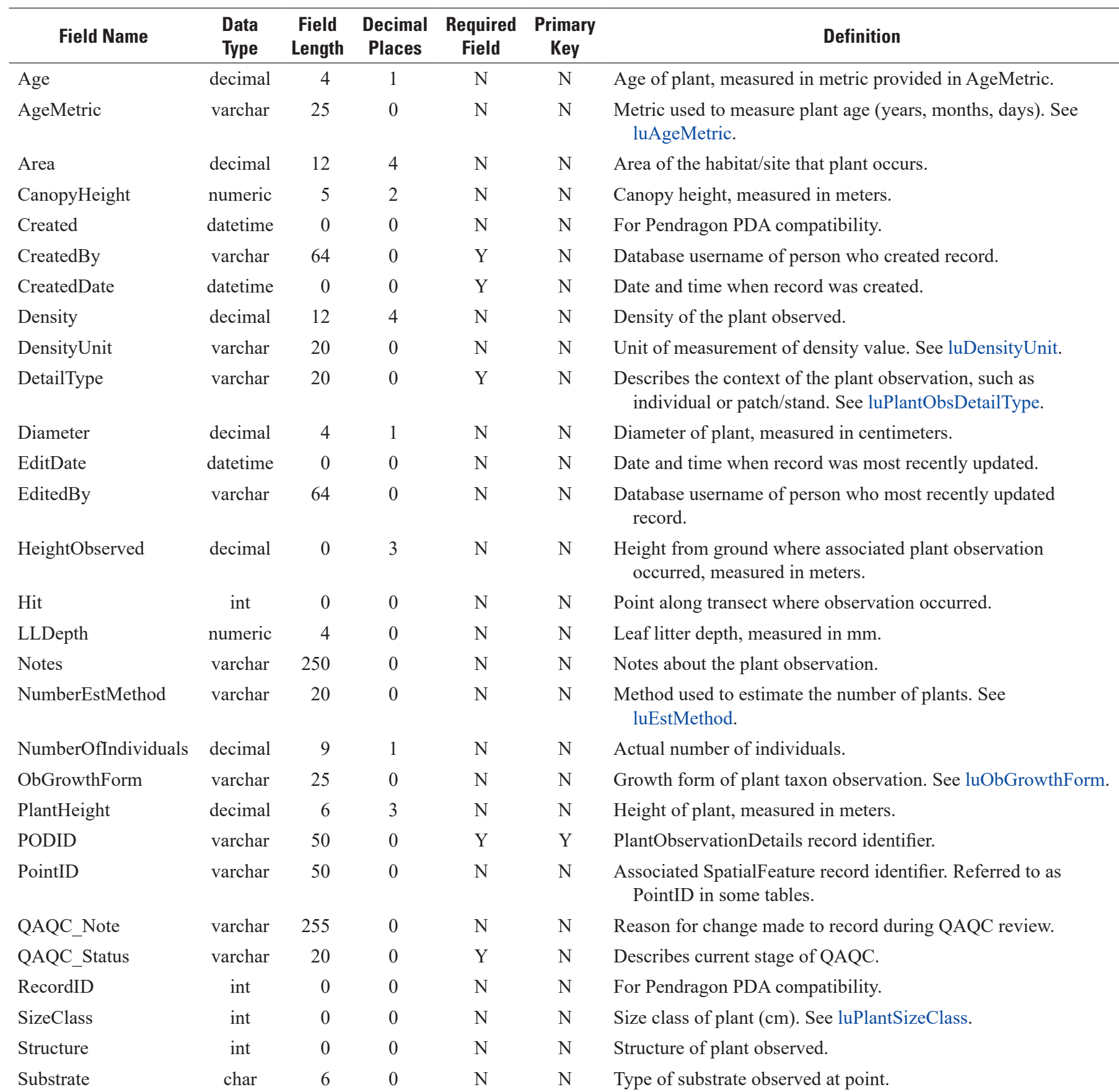


PlantObservationDetails Table-Continued

\begin{tabular}{lcccccl}
\hline \multicolumn{1}{c}{ Field Name } & $\begin{array}{c}\text { Data } \\
\text { Type }\end{array}$ & $\begin{array}{c}\text { Field } \\
\text { Length }\end{array}$ & $\begin{array}{c}\text { Decimal } \\
\text { Places }\end{array}$ & $\begin{array}{c}\text { Required } \\
\text { Field }\end{array}$ & $\begin{array}{c}\text { Primary } \\
\text { Key }\end{array}$ & Definition \\
\hline TaxaObservationID & varchar & 50 & 0 & $\mathrm{Y}$ & $\mathrm{N}$ & Associated TaxaObservation record identifier. \\
UnitID & int & 0 & 0 & $\mathrm{~N}$ & $\mathrm{~N}$ & For Pendragon PDA compatibility. \\
UserName & varchar & 64 & 0 & $\mathrm{~N}$ & $\mathrm{~N}$ & For Pendragon PDA compatibility. \\
\hline
\end{tabular}

PopulationEstimates Table

\begin{tabular}{|c|c|c|c|c|c|c|}
\hline Field Name & $\begin{array}{l}\text { Data } \\
\text { Type }\end{array}$ & $\begin{array}{c}\text { Field } \\
\text { Length }\end{array}$ & $\begin{array}{l}\text { Decimal } \\
\text { Places }\end{array}$ & $\begin{array}{l}\text { Required } \\
\text { Field }\end{array}$ & $\begin{array}{l}\text { Primary } \\
\text { Key }\end{array}$ & Definition \\
\hline ARMITier & varchar & 10 & 0 & $\mathrm{~N}$ & $\mathrm{~N}$ & $\begin{array}{l}\text { The ARMI program can record from which tier of the conceptual } \\
\text { pyramid the associated estimates are linked. }\end{array}$ \\
\hline CreatedBy & varchar & 64 & 0 & $\mathrm{Y}$ & $\mathrm{N}$ & Database username of person who created record. \\
\hline CreatedDate & datetime & 0 & 0 & $\mathrm{Y}$ & $\mathrm{N}$ & Date and time when record was created. \\
\hline EditDate & datetime & 0 & 0 & $\mathrm{~N}$ & $\mathrm{~N}$ & Date and time when record was most recently updated. \\
\hline IncludeOnWebsite & bit & 0 & 0 & Y & $\mathrm{N}$ & $\begin{array}{l}\text { Flag to indicate if this Project data should be included on ARMI } \\
\text { website. }\end{array}$ \\
\hline InferenceArea & varchar & 50 & 0 & $\mathrm{Y}$ & $\mathrm{N}$ & The site that represents the area of inference for the estimates. \\
\hline Notes & varchar & 250 & 0 & $\mathrm{~N}$ & $\mathrm{~N}$ & Notes. \\
\hline QAQC_Note & varchar & 255 & 0 & $\mathrm{~N}$ & $\mathrm{~N}$ & Reason for change made to record during QAQC review. \\
\hline QAQC_Status & varchar & 20 & 0 & Y & $\mathrm{N}$ & Describes current stage of QAQC. \\
\hline Status & varchar & 20 & 0 & $\mathrm{~N}$ & $\mathrm{~N}$ & $\begin{array}{l}\text { Description of current stage of this population estimate, such as } \\
\text { planned, active, non-active, etc. }\end{array}$ \\
\hline StudySite & varchar & 50 & 0 & $\mathrm{~N}$ & $\mathrm{~N}$ & $\begin{array}{l}\text { Name of the site where the survey(s) occurred. If multiple sites } \\
\text { were surveyed, the site that represents the sub-sites collectively } \\
\text { is represented here, and all the sub-sites are linked to this } \\
\text { collective site via the Site_Site table. }\end{array}$ \\
\hline $\begin{array}{l}\text { SurveyDescription } \\
\text { ID }\end{array}$ & int & 0 & 0 & $\mathrm{~N}$ & $\mathrm{~N}$ & $\begin{array}{l}\text { If an Estimate is developed from a single study, as opposed to a } \\
\text { project, the SurveyDescriptionID for the study is recorded here }\end{array}$ \\
\hline
\end{tabular}

Project_Keywords Table

\begin{tabular}{lcccccl}
\hline Field Name & $\begin{array}{c}\text { Data } \\
\text { Type }\end{array}$ & $\begin{array}{c}\text { Field } \\
\text { Length }\end{array}$ & $\begin{array}{c}\text { Decimal } \\
\text { Places }\end{array}$ & $\begin{array}{c}\text { Required } \\
\text { Field }\end{array}$ & $\begin{array}{c}\text { Primary } \\
\text { Key }\end{array}$ & Definition \\
\hline CreatedBy & varchar & 64 & 0 & $\mathrm{Y}$ & $\mathrm{N}$ & Database username of person who created record. \\
CreatedDate & datetime & 0 & 0 & $\mathrm{Y}$ & $\mathrm{N}$ & Date and time when record was created. \\
EditDate & datetime & 0 & 0 & $\mathrm{~N}$ & $\mathrm{~N}$ & Date and time when record was most recently updated. \\
EditedBy & varchar & 64 & 0 & $\mathrm{~N}$ & $\mathrm{~N}$ & Database username of person who most recently updated record. \\
KeywordID & bigint & 19 & 0 & $\mathrm{Y}$ & $\mathrm{Y}$ & Related Keywords record identifier. \\
ProjectID & varchar & 12 & 0 & $\mathrm{Y}$ & $\mathrm{Y}$ & Related ProjectID record identifier. \\
\hline
\end{tabular}


Project_Project Table

\begin{tabular}{lcccccl}
\hline \multicolumn{1}{c}{ Field Name } & $\begin{array}{c}\text { Data } \\
\text { Type }\end{array}$ & $\begin{array}{c}\text { Field } \\
\text { Length }\end{array}$ & $\begin{array}{c}\text { Decimal } \\
\text { Places }\end{array}$ & $\begin{array}{c}\text { Required } \\
\text { Field }\end{array}$ & $\begin{array}{c}\text { Primary } \\
\text { Key }\end{array}$ & Definition \\
\hline ChildProjectID & varchar & 12 & 0 & $\mathrm{Y}$ & $\mathrm{Y}$ & ProjectID of child project (sub-project). \\
CreatedBy & varchar & 64 & 0 & $\mathrm{Y}$ & $\mathrm{N}$ & Database username of person who created record. \\
CreatedDate & datetime & 0 & 0 & $\mathrm{Y}$ & $\mathrm{N}$ & Date and time when record was created. \\
EditDate & datetime & 0 & 0 & $\mathrm{~N}$ & $\mathrm{~N}$ & Date and time when record was most recently updated. \\
EditedBy & varchar & 64 & 0 & $\mathrm{~N}$ & $\mathrm{~N}$ & Database username of person who most recently updated record. \\
Notes & varchar & 250 & 0 & $\mathrm{~N}$ & $\mathrm{~N}$ & Notes about the relationship between the associated projects. \\
ParentProjectID & varchar & 12 & 0 & $\mathrm{Y}$ & $\mathrm{Y}$ & Project ID of parent project (main project). \\
ProjectProjectID & bigint & 19 & 0 & $\mathrm{Y}$ & $\mathrm{Y}$ & Project_Project record identifier. \\
\hline
\end{tabular}

\section{ProjectID Table}

\begin{tabular}{lcrcccl}
\hline \multicolumn{1}{c}{ Field Name } & $\begin{array}{c}\text { Data } \\
\text { Type }\end{array}$ & $\begin{array}{c}\text { Field } \\
\text { Length }\end{array}$ & $\begin{array}{c}\text { Decimal } \\
\text { Places }\end{array}$ & $\begin{array}{c}\text { Required } \\
\text { Field }\end{array}$ & $\begin{array}{c}\text { Primary } \\
\text { Key }\end{array}$ & Definition \\
\hline Category & varchar & 25 & 0 & $\mathrm{~N}$ & $\mathrm{~N}$ & Type/category of project. See luProjectCategory. \\
CreatedBy & varchar & 64 & 0 & $\mathrm{Y}$ & $\mathrm{N}$ & Database username of person who created record. \\
CreatedDate & datetime & 0 & 0 & $\mathrm{Y}$ & $\mathrm{N}$ & Date and time when record was created. \\
Description & varchar & 1,000 & 0 & $\mathrm{~N}$ & $\mathrm{~N}$ & Description of the project. \\
EditDate & datetime & 0 & 0 & $\mathrm{~N}$ & $\mathrm{~N}$ & Date and time when record was most recently updated. \\
EditedBy & varchar & 64 & 0 & $\mathrm{~N}$ & $\mathrm{~N}$ & Database username of person who most recently updated record. \\
Goals & varchar & 250 & 0 & $\mathrm{~N}$ & $\mathrm{~N}$ & Goals of the project. \\
Internal & bit & 0 & 0 & $\mathrm{~N}$ & $\mathrm{~N}$ & Y/N. Internal project. \\
Notes & varchar & 250 & 0 & $\mathrm{~N}$ & $\mathrm{~N}$ & Notes about the project. \\
ProjectID & varchar & 12 & 0 & $\mathrm{Y}$ & $\mathrm{Y}$ & Project record identifier. \\
ProjectName & varchar & 100 & 0 & $\mathrm{Y}$ & $\mathrm{N}$ & Official name of the project. \\
ProjectRecordPOC & int & 0 & 0 & $\mathrm{~N}$ & $\mathrm{~N}$ & Affiliate ID of person who created this record and has knowledge \\
& & & & & & of its purpose. \\
\hline
\end{tabular}

\section{Protocol Table}

\begin{tabular}{lcrcccl}
\multicolumn{1}{c}{ Field Name } & $\begin{array}{c}\text { Data } \\
\text { Type }\end{array}$ & $\begin{array}{r}\text { Field } \\
\text { Length }\end{array}$ & $\begin{array}{c}\text { Decimal } \\
\text { Places }\end{array}$ & $\begin{array}{c}\text { Required } \\
\text { Field }\end{array}$ & $\begin{array}{c}\text { Primary } \\
\text { Key }\end{array}$ & Definition \\
CitationID & varchar & 50 & 0 & $\mathrm{~N}$ & $\mathrm{~N}$ & Citation record identifier. \\
CreatedBy & varchar & 64 & 0 & $\mathrm{Y}$ & $\mathrm{N}$ & Database username of person who created record. \\
CreatedDate & datetime & 0 & 0 & $\mathrm{Y}$ & $\mathrm{N}$ & Date and time when record was created. \\
EditDate & datetime & 0 & 0 & $\mathrm{~N}$ & $\mathrm{~N}$ & Date and time when record was most recently updated. \\
EditedBy & varchar & 64 & 0 & $\mathrm{~N}$ & $\mathrm{~N}$ & Database username of person who most recently updated record. \\
EFIID & bigint & 19 & 0 & $\mathrm{~N}$ & $\mathrm{~N}$ & Associated ExternalFileInfo record identifier. \\
IsSubProtocol & char & 1 & 0 & $\mathrm{~N}$ & $\mathrm{~N}$ & Y/N. Is this protocol a sub-protocol of a larger protocol? \\
ParentProtocolID & int & 0 & 0 & $\mathrm{~N}$ & $\mathrm{~N}$ & Protocol record identifier of parent protocol. \\
ProtocolDescription & varchar & 1,000 & 0 & $\mathrm{~N}$ & $\mathrm{~N}$ & General description of protocol. \\
ProtocolFull & image & 2,147, & 0 & $\mathrm{~N}$ & $\mathrm{~N}$ & Complete protocol document. \\
& & 483,647 & & & & \\
ProtocolID & int & 0 & 0 & $\mathrm{Y}$ & $\mathrm{Y}$ & Protocol record identifier. \\
ProtocolName & varchar & 100 & 0 & $\mathrm{Y}$ & $\mathrm{N}$ & Official name of protocol.
\end{tabular}




\section{Protocol Table-Continued}

\begin{tabular}{lcccccl}
\hline \multicolumn{1}{c}{ Field Name } & $\begin{array}{c}\text { Data } \\
\text { Type }\end{array}$ & $\begin{array}{c}\text { Field } \\
\text { Length }\end{array}$ & $\begin{array}{c}\text { Decimal } \\
\text { Places }\end{array}$ & $\begin{array}{c}\text { Required } \\
\text { Field }\end{array}$ & $\begin{array}{c}\text { Primary } \\
\text { Key }\end{array}$ & Definition \\
\hline $\begin{array}{l}\text { ProtocolRecordVal- } \\
\text { idated }\end{array}$ & bit & 0 & 0 & $\mathrm{~N}$ & $\mathrm{~N}$ & Denotes whether the protocol has been validated by staff. \\
$\begin{array}{l}\text { ProtocolSource } \\
\text { Reference }\end{array}$ & varchar & 100 & 0 & $\mathrm{~N}$ & $\mathrm{~N}$ & Citation of protocol. \\
$\begin{array}{l}\text { ProtocolURL } \\
\text { SourceOfRecord }\end{array}$ & varchar & 255 & 0 & $\mathrm{~N}$ & $\mathrm{~N}$ & Website address of protocol document. \\
$\begin{array}{l}\text { Version } \\
\text { VersionByAffiliate }\end{array}$ & varchar & 50 & 0 & $\mathrm{~N}$ & $\mathrm{~N}$ & Number or indicator of version. \\
$\quad$ ID & & 0 & 0 & $\mathrm{~N}$ & $\mathrm{~N}$ & Person who wrote the version of the protocol. \\
VersionDate & datetime & 0 & 0 & $\mathrm{~N}$ & $\mathrm{~N}$ & Date this version of the protocol was published. \\
\hline
\end{tabular}

\section{Refugia Table}

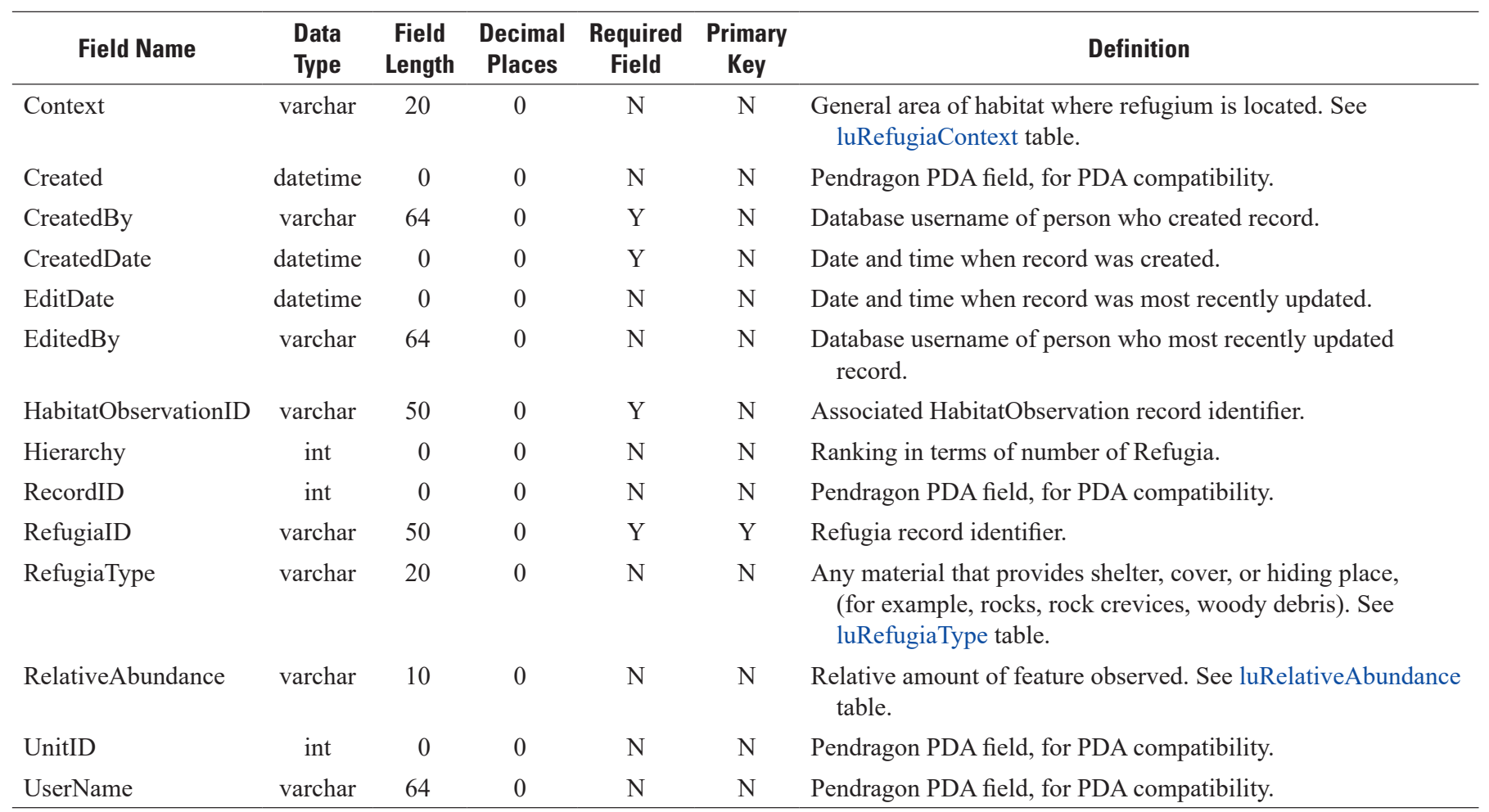

\section{Reserve Table}

$\begin{array}{lcccccc}\text { Field Name } & \begin{array}{c}\text { Data } \\ \text { Type }\end{array} & \begin{array}{c}\text { Field } \\ \text { Length }\end{array} & \begin{array}{c}\text { Decimal } \\ \text { Places }\end{array} & \begin{array}{c}\text { Required } \\ \text { Field }\end{array} & \begin{array}{c}\text { Primary } \\ \text { Key }\end{array} & \text { Definition } \\ \text { CreatedBy } & \text { varchar } & 64 & 0 & \mathrm{Y} & \mathrm{N} & \text { Database username of person who created record. } \\ \text { CreatedDate } & \text { datetime } & 0 & 0 & \mathrm{Y} & \mathrm{N} & \text { Date and time when record was created. } \\ \text { EditDate } & \text { datetime } & 0 & 0 & \mathrm{~N} & \mathrm{~N} & \text { Date and time when record was most recently updated. } \\ \text { EditedBy } & \text { varchar } & 64 & 0 & \mathrm{~N} & \mathrm{~N} & \text { Database username of person who most recently updated record. } \\ \text { Note } & \text { varchar } & 255 & 0 & \mathrm{~N} & \mathrm{~N} & \text { Note about the reserve. }\end{array}$


Reserve Table-Continued

\begin{tabular}{lccccccl}
\hline \multicolumn{1}{c}{ Field Name } & $\begin{array}{c}\text { Data } \\
\text { Type }\end{array}$ & $\begin{array}{c}\text { Field } \\
\text { Length }\end{array}$ & $\begin{array}{c}\text { Decimal } \\
\text { Places }\end{array}$ & $\begin{array}{c}\text { Required } \\
\text { Field }\end{array}$ & $\begin{array}{c}\text { Primary } \\
\text { Key }\end{array}$ & Definition \\
\hline QAQC_Note & varchar & 255 & 0 & $\mathrm{~N}$ & $\mathrm{~N}$ & Reason for change made to record during QAQC review. \\
QAQC_Status & varchar & 20 & 0 & $\mathrm{Y}$ & $\mathrm{N}$ & Describes current stage of QAQC. \\
Reserve & varchar & 50 & 0 & $\mathrm{Y}$ & $\mathrm{N}$ & Name of the reserve. \\
ReserveID & int & 0 & 0 & $\mathrm{Y}$ & $\mathrm{Y}$ & Reserve record identifier. \\
\hline
\end{tabular}

Reserve_Site Table

\begin{tabular}{lccccccl}
\hline Field Name & $\begin{array}{c}\text { Data } \\
\text { Type }\end{array}$ & $\begin{array}{c}\text { Field } \\
\text { Length }\end{array}$ & $\begin{array}{c}\text { Decimal } \\
\text { Places }\end{array}$ & $\begin{array}{c}\text { Required } \\
\text { Field }\end{array}$ & $\begin{array}{c}\text { Primary } \\
\text { Key }\end{array}$ & Definition \\
\hline CreatedBy & varchar & 64 & 0 & $\mathrm{Y}$ & $\mathrm{N}$ & Database username of person who created record. \\
CreatedDate & datetime & 0 & 0 & $\mathrm{Y}$ & $\mathrm{N}$ & Date and time when record was created. \\
EditDate & datetime & 0 & 0 & $\mathrm{~N}$ & $\mathrm{~N}$ & Date and time when record was most recently updated. \\
EditedBy & varchar & 64 & 0 & $\mathrm{~N}$ & $\mathrm{~N}$ & Database username of person who most recently updated record. \\
ReserveID & int & 0 & 0 & $\mathrm{Y}$ & $\mathrm{Y}$ & Associated Reserve record identifier. \\
SiteID & varchar & 50 & 0 & $\mathrm{Y}$ & $\mathrm{Y}$ & Associated Site record identifier. \\
\hline
\end{tabular}

RoadsTrails Table

\begin{tabular}{|c|c|c|c|c|c|c|}
\hline Field Name & $\begin{array}{l}\text { Data } \\
\text { Type }\end{array}$ & $\begin{array}{l}\text { Field } \\
\text { Length }\end{array}$ & $\begin{array}{l}\text { Decimal } \\
\text { Places }\end{array}$ & $\begin{array}{l}\text { Required } \\
\text { Field }\end{array}$ & $\begin{array}{l}\text { Primary } \\
\text { Key }\end{array}$ & Definition \\
\hline Access & varchar & 30 & 0 & $\mathrm{~N}$ & $\mathrm{~N}$ & $\begin{array}{l}\text { Description of how and why road/trail is used. See } \\
\text { luRoadTrailsAccess. }\end{array}$ \\
\hline Created & datetime & 0 & 0 & $\mathrm{~N}$ & $\mathrm{~N}$ & Pendragon PDA field, for PDA compatibility. \\
\hline CreatedBy & varchar & 64 & 0 & Y & $\mathrm{N}$ & Database username of person who created record. \\
\hline CreatedDate & datetime & 0 & 0 & $\mathrm{Y}$ & $\mathrm{N}$ & Date and time when record was created. \\
\hline Edge & varchar & 30 & 0 & $\mathrm{~N}$ & $\mathrm{~N}$ & $\begin{array}{l}\text { Composition of interface between the road/trail and the site. See } \\
\text { luRoadTrailsEdge. }\end{array}$ \\
\hline EditDate & datetime & 0 & 0 & $\mathrm{~N}$ & $\mathrm{~N}$ & Date and time when record was most recently updated. \\
\hline EditedBy & varchar & 64 & 0 & $\mathrm{~N}$ & $\mathrm{~N}$ & Database username of person who most recently updated record. \\
\hline PercentAreaOfSite & int & 0 & 0 & $\mathrm{~N}$ & $\mathrm{~N}$ & Percent of surface of site covered by road/trail. \\
\hline RecordID & int & 0 & 0 & $\mathrm{~N}$ & $\mathrm{~N}$ & Pendragon PDA field, for PDA compatibility. \\
\hline RoadsTrailsID & varchar & 50 & 0 & Y & Y & RoadsTrails record identifier. \\
\hline SiteID & varchar & 50 & 0 & $\mathrm{~N}$ & $\mathrm{~N}$ & Associated Site record identifier. \\
\hline Type & varchar & 30 & 0 & $\mathrm{~N}$ & $\mathrm{~N}$ & $\begin{array}{l}\text { General type and construction of road/trail. See } \\
\text { luRoadTrailsType. }\end{array}$ \\
\hline UnitID & int & 0 & 0 & $\mathrm{~N}$ & $\mathrm{~N}$ & Pendragon PDA field, for PDA compatibility. \\
\hline UserName & varchar & 64 & 0 & $\mathrm{~N}$ & $\mathrm{~N}$ & Pendragon PDA field, for PDA compatibility. \\
\hline Width & decimal & 3 & 1 & $\mathrm{~N}$ & $\mathrm{~N}$ & Width of road or trail, measured in meters. \\
\hline
\end{tabular}


Site Table

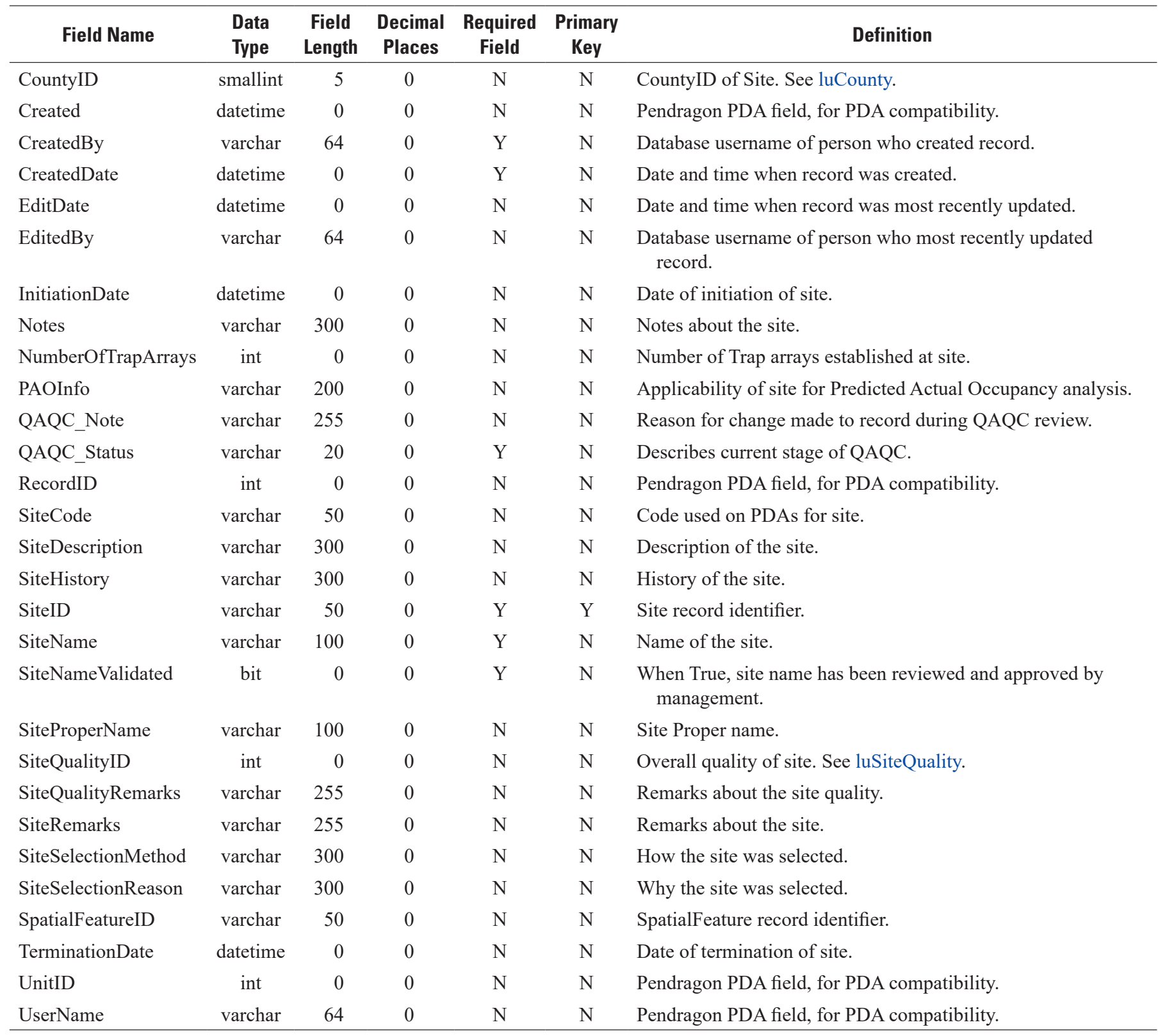

Site_Project Table

\begin{tabular}{lcccccl}
\hline $\begin{array}{c}\text { Field } \\
\text { Name }\end{array}$ & $\begin{array}{c}\text { Data } \\
\text { Type }\end{array}$ & $\begin{array}{c}\text { Field } \\
\text { Length }\end{array}$ & $\begin{array}{c}\text { Decimal } \\
\text { Places }\end{array}$ & $\begin{array}{c}\text { Required } \\
\text { Field }\end{array}$ & $\begin{array}{c}\text { Primary } \\
\text { Key }\end{array}$ & Definition \\
\hline ProjectID & varchar & 12 & 0 & $\mathrm{Y}$ & $\mathrm{Y}$ & Associated Project record identifier. \\
SiteID & varchar & 50 & 0 & $\mathrm{Y}$ & $\mathrm{Y}$ & Associated Site record identifier. \\
\hline
\end{tabular}

Site_Site Table

\begin{tabular}{lcccccl}
\hline \multicolumn{1}{c}{ Field Name } & $\begin{array}{c}\text { Data } \\
\text { Type }\end{array}$ & $\begin{array}{c}\text { Field } \\
\text { Length }\end{array}$ & $\begin{array}{c}\text { Decimal } \\
\text { Places }\end{array}$ & $\begin{array}{c}\text { Required } \\
\text { Field }\end{array}$ & $\begin{array}{c}\text { Primary } \\
\text { Key }\end{array}$ & Definition \\
\hline ChildSiteID & varchar & 50 & 0 & $\mathrm{Y}$ & $\mathrm{N}$ & Site record identifier for the child site. \\
Created & datetime & 0 & 0 & $\mathrm{~N}$ & $\mathrm{~N}$ & Pendragon PDA field, for PDA compatibility.
\end{tabular}


Site_Site Table-Continued

\begin{tabular}{lcccccl}
\hline \multicolumn{1}{c}{ Field Name } & $\begin{array}{c}\text { Data } \\
\text { Type }\end{array}$ & $\begin{array}{c}\text { Field } \\
\text { Length }\end{array}$ & $\begin{array}{c}\text { Decimal } \\
\text { Places }\end{array}$ & $\begin{array}{c}\text { Required } \\
\text { Field }\end{array}$ & $\begin{array}{c}\text { Primary } \\
\text { Key }\end{array}$ & Definition \\
\hline CreatedBy & varchar & 64 & 0 & $\mathrm{Y}$ & $\mathrm{N}$ & Database username of person who created record. \\
CreatedDate & datetime & 0 & 0 & $\mathrm{Y}$ & $\mathrm{N}$ & Date and time when record was created. \\
EditDate & datetime & 0 & 0 & $\mathrm{~N}$ & $\mathrm{~N}$ & Date and time when record was most recently updated. \\
EditedBy & varchar & 64 & 0 & $\mathrm{~N}$ & $\mathrm{~N}$ & Database username of person who most recently updated record. \\
Notes & varchar & 250 & 0 & $\mathrm{~N}$ & $\mathrm{~N}$ & Notes about the parent child site relationship. \\
ParentSiteID & varchar & 50 & 0 & $\mathrm{Y}$ & $\mathrm{N}$ & Site record identifier for the parent site. \\
RecordID & int & 0 & 0 & $\mathrm{~N}$ & $\mathrm{~N}$ & Pendragon PDA field, for PDA compatibility. \\
RelationshipType & varchar & 20 & 0 & $\mathrm{~N}$ & $\mathrm{~N}$ & Identifies the relationship between the parent and child site. See \\
& & & & & & luSiteRelationshipType. \\
SiteSiteID & bigint & 19 & 0 & $\mathrm{Y}$ & $\mathrm{Y}$ & Site_Site record identifier. \\
UnitID & int & 0 & 0 & $\mathrm{~N}$ & $\mathrm{~N}$ & Pendragon PDA field, for PDA compatibility. \\
UserName & varchar & 64 & 0 & $\mathrm{~N}$ & $\mathrm{~N}$ & Pendragon PDA field, for PDA compatibility.
\end{tabular}

\section{Site_SiteThreatType Table}

\begin{tabular}{lcccccl}
\hline \multicolumn{1}{c}{ Field Name } & $\begin{array}{c}\text { Data } \\
\text { Type }\end{array}$ & $\begin{array}{c}\text { Field } \\
\text { Length }\end{array}$ & $\begin{array}{c}\text { Decimal } \\
\text { Places }\end{array}$ & $\begin{array}{c}\text { Required } \\
\text { Field }\end{array}$ & $\begin{array}{c}\text { Primary } \\
\text { Key }\end{array}$ & Definition \\
\hline Created & datetime & 0 & 0 & $\mathrm{~N}$ & $\mathrm{~N}$ & Pendragon PDA field, for PDA compatibility. \\
CreatedBy & varchar & 64 & 0 & $\mathrm{Y}$ & $\mathrm{N}$ & Database username of person who created record. \\
CreatedDate & datetime & 0 & 0 & $\mathrm{Y}$ & $\mathrm{N}$ & Date and time when record was created. \\
EditDate & datetime & 0 & 0 & $\mathrm{~N}$ & $\mathrm{~N}$ & Date and time when record was most recently updated. \\
EditedBy & varchar & 64 & 0 & $\mathrm{~N}$ & $\mathrm{~N}$ & Database username of person who most recently updated record. \\
RecordID & int & 0 & 0 & $\mathrm{~N}$ & $\mathrm{~N}$ & Pendragon PDA field, for PDA compatibility. \\
SiteID & varchar & 50 & 0 & $\mathrm{Y}$ & $\mathrm{Y}$ & Site record identifier. \\
SiteThreatRemarks & varchar & 255 & 0 & $\mathrm{~N}$ & $\mathrm{~N}$ & Remarks about the threat to the site. \\
SiteThreatTypeID & int & 0 & 0 & $\mathrm{Y}$ & $\mathrm{Y}$ & Threat to the site. See luSiteThreatType table. \\
UnitID & int & 0 & 0 & $\mathrm{~N}$ & $\mathrm{~N}$ & Pendragon PDA field, for PDA compatibility. \\
UserName & varchar & 64 & 0 & $\mathrm{~N}$ & $\mathrm{~N}$ & Pendragon PDA field, for PDA compatibility. \\
\hline
\end{tabular}

\section{SiteMaintenance Table}

\begin{tabular}{lcccccl}
\hline \multicolumn{1}{c}{ Field Name } & $\begin{array}{c}\text { Data } \\
\text { Type }\end{array}$ & $\begin{array}{c}\text { Field } \\
\text { Length }\end{array}$ & $\begin{array}{c}\text { Decimal } \\
\text { Places }\end{array}$ & $\begin{array}{c}\text { Required } \\
\text { Field }\end{array}$ & $\begin{array}{c}\text { Primary } \\
\text { Key }\end{array}$ & Definition \\
\hline AmountNeeded & varchar & 100 & 0 & $\mathrm{~N}$ & $\mathrm{~N}$ & Can further define Maintenance Needed by quantifying it. \\
Created & datetime & 0 & 0 & $\mathrm{~N}$ & $\mathrm{~N}$ & Pendragon PDA field, for PDA compatibility. \\
CreatedBy & varchar & 64 & 0 & $\mathrm{Y}$ & $\mathrm{N}$ & Database username of person who created record. \\
CreatedDate & datetime & 0 & 0 & $\mathrm{Y}$ & $\mathrm{N}$ & Date and time when record was created. \\
DateClosed & datetime & 0 & 0 & $\mathrm{~N}$ & $\mathrm{~N}$ & Date site was closed due to maintenance need. \\
DateNeeded & datetime & 0 & 0 & $\mathrm{~N}$ & $\mathrm{~N}$ & Date the maintenance is needed. \\
DateOpened & datetime & 0 & 0 & $\mathrm{~N}$ & $\mathrm{~N}$ & Date site was opened after maintenance. \\
DatePerformed & datetime & 0 & 0 & $\mathrm{~N}$ & $\mathrm{~N}$ & Date the maintenance was performed. \\
EditDate & datetime & 0 & 0 & $\mathrm{~N}$ & $\mathrm{~N}$ & Date and time when record was most recently updated. \\
EditedBy & varchar & 64 & 0 & $\mathrm{~N}$ & $\mathrm{~N}$ & Database username of person who most recently updated \\
& & & & & & record. \\
MaintenanceNeeded & varchar & 200 & 0 & $\mathrm{~N}$ & $\mathrm{~N}$ & Description of the type of maintenance needed at the site.
\end{tabular}




\begin{tabular}{lcccccl}
\multicolumn{1}{c}{ SiteMaintenance Table-Continued } & & & \\
\multicolumn{1}{c}{ Field Name } & $\begin{array}{c}\text { Data } \\
\text { Type }\end{array}$ & $\begin{array}{c}\text { Field } \\
\text { Length }\end{array}$ & $\begin{array}{c}\text { Decimal } \\
\text { Places }\end{array}$ & $\begin{array}{c}\text { Required } \\
\text { Field }\end{array}$ & $\begin{array}{c}\text { Primary } \\
\text { Key }\end{array}$ & Definition \\
\hline MaintenancePerformed & varchar & 200 & 0 & $\mathrm{~N}$ & $\mathrm{~N}$ & Description of maintenance performed. \\
Notes & varchar & 250 & 0 & $\mathrm{~N}$ & $\mathrm{~N}$ & Notes about the maintenance record. \\
RecordID & int & 0 & 0 & $\mathrm{~N}$ & $\mathrm{~N}$ & Pendragon PDA field, for PDA compatibility. \\
SiteID & varchar & 50 & 0 & $\mathrm{~N}$ & $\mathrm{~N}$ & Associated Site record identifier. \\
SiteMaintenanceID & varchar & 50 & 0 & $\mathrm{Y}$ & $\mathrm{Y}$ & SiteMaintenance record identifier. \\
SpatialFeatureID & varchar & 50 & 0 & $\mathrm{~N}$ & $\mathrm{~N}$ & Associated SpatialFeature record identifier. \\
SurveyEventID & varchar & 50 & 0 & $\mathrm{~N}$ & $\mathrm{~N}$ & Associated SurveyEvent record identifier. \\
UnitID & int & 0 & 0 & $\mathrm{~N}$ & $\mathrm{~N}$ & Pendragon PDA field, for PDA compatibility. \\
UserName & varchar & 64 & 0 & $\mathrm{~N}$ & $\mathrm{~N}$ & Pendragon PDA field, for PDA compatibility.
\end{tabular}

\section{SiteMetadata Table}

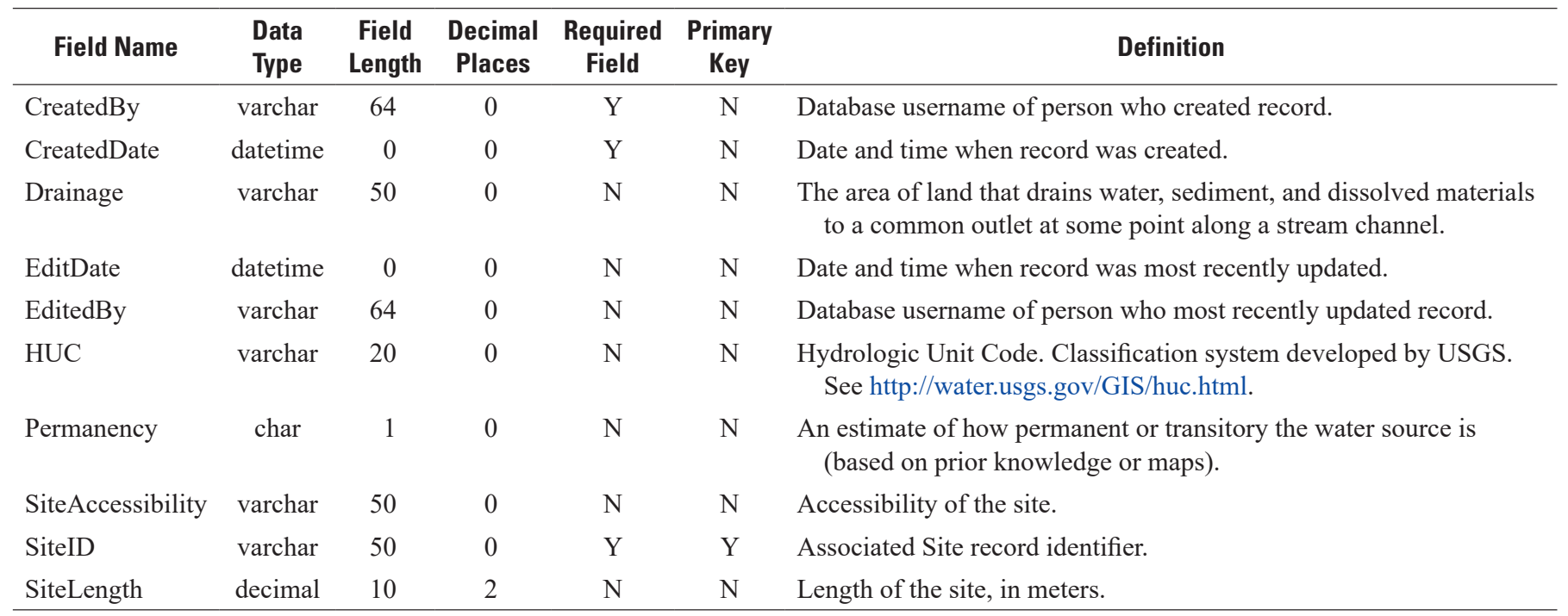

\section{SoilPhysicalObservation Table}

\begin{tabular}{lcccccl}
\hline \multicolumn{1}{c}{ Field Name } & $\begin{array}{c}\text { Data } \\
\text { Type }\end{array}$ & $\begin{array}{c}\text { Field } \\
\text { Length }\end{array}$ & $\begin{array}{c}\text { Decimal } \\
\text { Places }\end{array}$ & $\begin{array}{c}\text { Required } \\
\text { Field }\end{array}$ & $\begin{array}{c}\text { Primary } \\
\text { Key }\end{array}$ & Definition \\
\hline Clay & numeric & 8 & 4 & $\mathrm{~N}$ & $\mathrm{~N}$ & Amount of clay found within the soil. \\
Created & datetime & 0 & 0 & $\mathrm{~N}$ & $\mathrm{~N}$ & Pendragon PDA field, for PDA compatibility. \\
CreatedBy & varchar & 64 & 0 & $\mathrm{Y}$ & $\mathrm{N}$ & Database username of person who created record. \\
CreatedDate & datetime & 0 & 0 & $\mathrm{Y}$ & $\mathrm{N}$ & Date and time when record was created. \\
EditDate & datetime & 0 & 0 & $\mathrm{~N}$ & $\mathrm{~N}$ & Date and time when record was most recently updated. \\
EditedBy & varchar & 64 & 0 & $\mathrm{~N}$ & $\mathrm{~N}$ & Database username of person who most recently updated \\
& & & & & & record. \\
Method & varchar & 30 & 0 & $\mathrm{~N}$ & $\mathrm{~N}$ & Method used to analyze the soil. See luSoilMethod. \\
Notes & varchar & 255 & 0 & $\mathrm{~N}$ & $\mathrm{~N}$ & Notes about the soil physical observation. \\
ObservationDate & datetime & 0 & 0 & $\mathrm{~N}$ & $\mathrm{~N}$ & Date/time of the observation. \\
ObservedByAffiliateID & int & 0 & 0 & $\mathrm{~N}$ & $\mathrm{~N}$ & Affiliate who sampled/collected the soil. \\
OsmoticPotential & numeric & 8 & 4 & $\mathrm{~N}$ & $\mathrm{~N}$ & Osmotic potential of the soil sample. \\
PointID & varchar & 50 & 0 & $\mathrm{~N}$ & $\mathrm{~N}$ & Associated SpatialFeature record identifier.
\end{tabular}


SoilPhysicalObservation Table-Continued

\begin{tabular}{lcccccl}
\hline \multicolumn{1}{c}{ Field Name } & $\begin{array}{c}\text { Data } \\
\text { Type }\end{array}$ & $\begin{array}{c}\text { Field } \\
\text { Length }\end{array}$ & $\begin{array}{c}\text { Decimal } \\
\text { Places }\end{array}$ & $\begin{array}{c}\text { Required } \\
\text { Field }\end{array}$ & $\begin{array}{c}\text { Primary } \\
\text { Key }\end{array}$ & Definition \\
\hline QAQC_Note & varchar & 255 & 0 & $\mathrm{~N}$ & $\mathrm{~N}$ & Reason for change made to record during QAQC review. \\
QAQC_Status & varchar & 20 & 0 & $\mathrm{Y}$ & $\mathrm{N}$ & Describes current stage of QAQC. \\
RecordID & int & 0 & 0 & $\mathrm{~N}$ & $\mathrm{~N}$ & Pendragon PDA field, for PDA compatibility. \\
Sand & numeric & 8 & 4 & $\mathrm{~N}$ & $\mathrm{~N}$ & Amount of sand found within the soil. \\
SiblingID & varchar & 50 & 0 & $\mathrm{~N}$ & $\mathrm{~N}$ & Record identifier of associated record. \\
Silt & numeric & 8 & 4 & $\mathrm{~N}$ & $\mathrm{~N}$ & Amount of silt found within the soil. \\
SiteID & varchar & 50 & 0 & $\mathrm{Y}$ & $\mathrm{N}$ & Associated Site record identifier. \\
SoilDepth & numeric & 6 & 2 & $\mathrm{~N}$ & $\mathrm{~N}$ & Depth of soil, measured in centimeters. \\
SPOID & varchar & 50 & 0 & $\mathrm{Y}$ & $\mathrm{Y}$ & SoilPhysicalObservation record identifier. \\
SurveyEventID & varchar & 50 & 0 & $\mathrm{Y}$ & $\mathrm{N}$ & Associated SurveyEvent record identifier. \\
TextureClass & varchar & 20 & 0 & $\mathrm{~N}$ & $\mathrm{~N}$ & Texture classification of soil/soil sample. See luTextureClass. \\
Unit & varchar & 20 & 0 & $\mathrm{~N}$ & $\mathrm{~N}$ & Unit of measurement of soil sample. See luSoilUnit. \\
UnitID & int & 0 & 0 & $\mathrm{~N}$ & $\mathrm{~N}$ & Pendragon PDA field, for PDA compatibility. \\
UserName & varchar & 64 & 0 & $\mathrm{~N}$ & $\mathrm{~N}$ & Pendragon PDA field, for PDA compatibility. \\
\hline
\end{tabular}

\section{SourceXY Table}

\begin{tabular}{|c|c|c|c|c|c|c|}
\hline Field Name & $\begin{array}{l}\text { Data } \\
\text { Type }\end{array}$ & $\begin{array}{c}\text { Field } \\
\text { Length }\end{array}$ & $\begin{array}{l}\text { Decimal } \\
\text { Places }\end{array}$ & $\begin{array}{l}\text { Required } \\
\text { Field }\end{array}$ & $\begin{array}{c}\text { Primary } \\
\text { Key }\end{array}$ & Definition \\
\hline Created & datetime & 0 & 0 & $\mathrm{~N}$ & $\mathrm{~N}$ & Pendragon PDA field, for PDA compatibility. \\
\hline CreatedDate & datetime & 0 & 0 & Y & $\mathrm{N}$ & Date and time when record was created. \\
\hline Datum & varchar & 20 & 0 & $\mathrm{~N}$ & $\mathrm{~N}$ & Name of datum used to represent shape and offset of coordinates. \\
\hline ElevationMeters & int & 0 & 0 & $\mathrm{~N}$ & $\mathrm{~N}$ & Elevation in meters. \\
\hline EPE & decimal & 5 & 2 & $\mathrm{~N}$ & $\mathrm{~N}$ & $\begin{array}{l}\text { Estimated Position Error. Error associated with the coordinates } \\
\text { determined by a GPS device, in meters. }\end{array}$ \\
\hline HDOP & varchar & 10 & 0 & $\mathrm{~N}$ & $\mathrm{~N}$ & $\begin{array}{l}\text { Horizontal Dilution of Precision. Calculated by a GPS device, } \\
\text { provides the strength of satellite geometry when determining the } \\
\text { GPS location, in meters. }\end{array}$ \\
\hline Position & char & 2 & 0 & $\mathrm{~N}$ & $\mathrm{~N}$ & Source position NMGA string. \\
\hline $\begin{array}{l}\text { ProxyRadius } \\
\text { Meters }\end{array}$ & int & 0 & 0 & $\mathrm{~N}$ & $\mathrm{~N}$ & $\begin{array}{l}\text { Dilution of coordinate precision used for reporting locations of } \\
\text { sensitive species. }\end{array}$ \\
\hline QAQC_Note & varchar & 255 & 0 & $\mathrm{~N}$ & $\mathrm{~N}$ & Reason for change made to record during QAQC review. \\
\hline RecordID & int & 0 & 0 & $\mathrm{~N}$ & $\mathrm{~N}$ & Pendragon PDA field, for PDA compatibility. \\
\hline SpatialFeatureID & varchar & 50 & 0 & Y & $\mathrm{Y}$ & $\begin{array}{l}\text { SpatialFeature record identifier. Referred to as PointID in some } \\
\text { tables. }\end{array}$ \\
\hline UnitID & int & 0 & 0 & $\mathrm{~N}$ & $\mathrm{~N}$ & Pendragon PDA field, for PDA compatibility. \\
\hline UserName & varchar & 64 & 0 & $\mathrm{~N}$ & $\mathrm{~N}$ & Pendragon PDA field, for PDA compatibility. \\
\hline
\end{tabular}


SpatialFeature Table

\begin{tabular}{|c|c|c|c|c|c|c|}
\hline Field Name & $\begin{array}{l}\text { Data } \\
\text { Type }\end{array}$ & $\begin{array}{l}\text { Field } \\
\text { Length }\end{array}$ & $\begin{array}{l}\text { Decimal } \\
\text { Places }\end{array}$ & $\begin{array}{l}\text { Required } \\
\text { Field }\end{array}$ & $\begin{array}{l}\text { Primary } \\
\text { Key }\end{array}$ & Definition \\
\hline Area & decimal & 14 & 2 & $\mathrm{~N}$ & $\mathrm{~N}$ & For polygons, the area measured in square meters. \\
\hline CreatedBy & varchar & 64 & 0 & $\mathrm{Y}$ & $\mathrm{N}$ & Database username of person who created record. \\
\hline CreatedDate & datetime & 0 & 0 & Y & $\mathrm{N}$ & Date and time when record was created. \\
\hline EditedBy & varchar & 64 & 0 & $\mathrm{~N}$ & $\mathrm{~N}$ & Database username of person who most recently updated record. \\
\hline $\begin{array}{l}\text { Historic_area } \\
\text { located }\end{array}$ & bit & 0 & 0 & $\mathrm{~N}$ & $\mathrm{~N}$ & $\mathrm{Y} / \mathrm{N}$. Was the historic area located? \\
\hline $\begin{array}{l}\text { Historic_- } \\
\text { description }\end{array}$ & bit & 0 & 0 & $\mathrm{~N}$ & $\mathrm{~N}$ & Y/N. Does a historic description of this feature exist? \\
\hline Notes & varchar & 250 & 0 & $\mathrm{~N}$ & $\mathrm{~N}$ & Notes about the spatial feature. \\
\hline RecordID & int & 0 & 0 & $\mathrm{~N}$ & $\mathrm{~N}$ & Pendragon PDA field, for PDA compatibility. \\
\hline SpatialFeatureID & varchar & 50 & 0 & $\mathrm{Y}$ & Y & $\begin{array}{l}\text { SpatialFeature record identifier. Referred to as PointID in some } \\
\text { tables. }\end{array}$ \\
\hline UnitID & int & 0 & 0 & $\mathrm{~N}$ & $\mathrm{~N}$ & Pendragon PDA field, for PDA compatibility. \\
\hline UserName & varchar & 64 & 0 & $\mathrm{~N}$ & $\mathrm{~N}$ & Pendragon PDA field, for PDA compatibility. \\
\hline WayPoint & varchar & 20 & 0 & $\mathrm{~N}$ & $\mathrm{~N}$ & Waypoint identifier. \\
\hline
\end{tabular}

\section{SpatialFeature_CLDB Table}

\begin{tabular}{|c|c|c|c|c|c|c|}
\hline Field Name & $\begin{array}{l}\text { Data } \\
\text { Type }\end{array}$ & $\begin{array}{l}\text { Field } \\
\text { Length }\end{array}$ & $\begin{array}{l}\text { Decimal } \\
\text { Places }\end{array}$ & $\begin{array}{l}\text { Required } \\
\text { Field }\end{array}$ & $\begin{array}{l}\text { Primary } \\
\text { Key }\end{array}$ & Definition \\
\hline CreatedBy & varchar & 64 & 0 & $\mathrm{Y}$ & $\mathrm{N}$ & Database username of person who created record. \\
\hline DateRelevant & datetime & 0 & 0 & $\mathrm{~N}$ & $\mathrm{~N}$ & $\begin{array}{l}\text { The date the decision was made that this SpatialFeatureID should be } \\
\text { linked to this LandID }\end{array}$ \\
\hline EditDate & datetime & 0 & 0 & $\mathrm{~N}$ & $\mathrm{~N}$ & Date and time when record was most recently updated. \\
\hline LandID & varchar & 10 & 0 & $\mathrm{Y}$ & $\mathrm{N}$ & $\begin{array}{l}\text { Link to locally managed Conserved Lands database (CLDB); } \\
\text { specifically the CONSERVED_LAND table's unique LandID } \\
\text { field. }\end{array}$ \\
\hline Method & varchar & 30 & 0 & $\mathrm{~N}$ & $\mathrm{~N}$ & $\begin{array}{l}\text { The method by which the association between SpatialFeatureID and } \\
\text { LandID was made. See luSFCLDBMethod. }\end{array}$ \\
\hline
\end{tabular}




\section{SpeciesHabitatFeature Table}

\begin{tabular}{|c|c|c|c|c|c|c|}
\hline Field Name & $\begin{array}{l}\text { Data } \\
\text { Type }\end{array}$ & $\begin{array}{l}\text { Field } \\
\text { Length }\end{array}$ & $\begin{array}{l}\text { Decimal } \\
\text { Places }\end{array}$ & $\begin{array}{l}\text { Required } \\
\text { Field }\end{array}$ & $\begin{array}{l}\text { Primary } \\
\text { Key }\end{array}$ & Definition \\
\hline Created & datetime & 0 & 0 & $\mathrm{~N}$ & $\mathrm{~N}$ & Pendragon PDA field, for PDA compatibility. \\
\hline CreatedDate & datetime & 0 & 0 & $\mathrm{Y}$ & $\mathrm{N}$ & Date and time when record was created. \\
\hline EditDate & datetime & 0 & 0 & $\mathrm{~N}$ & $\mathrm{~N}$ & Date and time when record was most recently updated. \\
\hline FeatureCount & int & 0 & 0 & $\mathrm{~N}$ & $\mathrm{~N}$ & Number of species-specific features observed at site. \\
\hline FeatureRange & varchar & 10 & 0 & $\mathrm{~N}$ & $\mathrm{~N}$ & $\begin{array}{l}\text { Relative amount of feature at site. See luRelativeAbundance } \\
\text { table. }\end{array}$ \\
\hline FeatureType & varchar & 20 & 0 & $\mathrm{Y}$ & $\mathrm{N}$ & $\begin{array}{l}\text { Type of feature being used or has potential to be used by } \\
\text { observed species. See luFeatureType table. }\end{array}$ \\
\hline Notes & varchar & 255 & 0 & $\mathrm{~N}$ & $\mathrm{~N}$ & Notes about the species-specific habitat feature observation. \\
\hline PotentialNestSites & varchar & 10 & 0 & $\mathrm{~N}$ & $\mathrm{~N}$ & $\begin{array}{l}\text { Relative abundance of potential nesting sites for species. See } \\
\text { luRelativeAbundance table. }\end{array}$ \\
\hline QAQC_Note & varchar & 255 & 0 & $\mathrm{~N}$ & $\mathrm{~N}$ & Reason for change made to record during QAQC review. \\
\hline QAQC_Status & varchar & 20 & 0 & $\mathrm{Y}$ & $\mathrm{N}$ & Describes current stage of QAQC. \\
\hline RecordID & int & 0 & 0 & $\mathrm{~N}$ & $\mathrm{~N}$ & Pendragon PDA field, for PDA compatibility. \\
\hline SpeciesHabitatFeatureID & varchar & 50 & 0 & $\mathrm{Y}$ & $\mathrm{Y}$ & SpeciesHabitatFeature record identifier. \\
\hline TaxaID & varchar & 25 & 0 & $\mathrm{Y}$ & $\mathrm{N}$ & $\begin{array}{l}\text { Taxonomic Serial Number (www.itis.gov) of species related } \\
\text { to habitat feature. }\end{array}$ \\
\hline
\end{tabular}

\section{Spheroid Table}

\begin{tabular}{lccccccl}
\hline $\begin{array}{c}\text { Field } \\
\text { Name }\end{array}$ & $\begin{array}{c}\text { Data } \\
\text { Type }\end{array}$ & $\begin{array}{c}\text { Field } \\
\text { Length }\end{array}$ & $\begin{array}{c}\text { Decimal } \\
\text { Places }\end{array}$ & $\begin{array}{c}\text { Required } \\
\text { Field }\end{array}$ & $\begin{array}{c}\text { Primary } \\
\text { Key }\end{array}$ & Definition \\
\hline SID & char & 1 & 0 & Y & Y & Spheroid record identifier. & \\
Spheroid & varchar & 15 & 0 & Y & N & Spheroid reference for site coordinates. \\
\hline
\end{tabular}

\section{Stakeholders Table}

\begin{tabular}{lcccccl}
\hline \multicolumn{1}{c}{ Field Name } & $\begin{array}{c}\text { Data } \\
\text { Type }\end{array}$ & $\begin{array}{c}\text { Field } \\
\text { Length }\end{array}$ & $\begin{array}{c}\text { Decimal } \\
\text { Places }\end{array}$ & $\begin{array}{c}\text { Required } \\
\text { Field }\end{array}$ & $\begin{array}{c}\text { Primary } \\
\text { Key }\end{array}$ & Definition \\
\hline CreatedBy & varchar & 64 & 0 & $\mathrm{Y}$ & $\mathrm{N}$ & Database username of person who created record. \\
CreatedDate & datetime & 0 & 0 & $\mathrm{Y}$ & $\mathrm{N}$ & Date and time when record was created. \\
EditDate & datetime & 0 & 0 & $\mathrm{~N}$ & $\mathrm{~N}$ & Date and time when record was most recently updated. \\
EditedBy & varchar & 64 & 0 & $\mathrm{~N}$ & $\mathrm{~N}$ & Database username of person who most recently updated record. \\
Notes & varchar & 250 & 0 & $\mathrm{~N}$ & $\mathrm{~N}$ & Notes about the stakeholder. \\
PointOfContact & int & 0 & 0 & $\mathrm{~N}$ & $\mathrm{~N}$ & Specific person who is the point of contact for the stakeholder. \\
Stakeholder & int & 0 & 0 & $\mathrm{Y}$ & $\mathrm{N}$ & Entity or person who is a stakeholder in one or more projects. \\
StakeholderID & bigint & 19 & 0 & $\mathrm{Y}$ & $\mathrm{Y}$ & Stakeholder record identifier. \\
\hline
\end{tabular}


Stakeholder_Projects Table

\begin{tabular}{lcccccl}
\hline \multicolumn{1}{c}{ Field Name } & $\begin{array}{c}\text { Data } \\
\text { Type }\end{array}$ & $\begin{array}{c}\text { Field } \\
\text { Length }\end{array}$ & $\begin{array}{c}\text { Decimal } \\
\text { Places }\end{array}$ & $\begin{array}{c}\text { Required } \\
\text { Field }\end{array}$ & $\begin{array}{c}\text { Primary } \\
\text { Key }\end{array}$ & Definition \\
\hline CreatedBy & varchar & 64 & 0 & $\mathrm{Y}$ & $\mathrm{N}$ & Database username of person who created record. \\
CreatedDate & datetime & 0 & 0 & $\mathrm{Y}$ & $\mathrm{N}$ & Date and time when record was created. \\
EditDate & datetime & 0 & 0 & $\mathrm{~N}$ & $\mathrm{~N}$ & Date and time when record was most recently updated. \\
EditedBy & varchar & 64 & 0 & $\mathrm{~N}$ & $\mathrm{~N}$ & Database username of person who most recently updated record. \\
ProjectID & varchar & 12 & 0 & $\mathrm{~N}$ & $\mathrm{~N}$ & Associated ProjectID record identifier. \\
StakeholderID & bigint & 19 & 0 & $\mathrm{~N}$ & $\mathrm{~N}$ & Associated Stakeholders record identifier. \\
StakeholderProjectID & bigint & 19 & 0 & $\mathrm{Y}$ & $\mathrm{Y}$ & Stakeholder_Projects record identifier. \\
\hline
\end{tabular}

Study_Keywords Table

\begin{tabular}{lcccccl}
\hline \multicolumn{1}{c}{ Field Name } & $\begin{array}{c}\text { Data } \\
\text { Type }\end{array}$ & $\begin{array}{c}\text { Field } \\
\text { Length }\end{array}$ & $\begin{array}{c}\text { Decimal } \\
\text { Places }\end{array}$ & $\begin{array}{c}\text { Required } \\
\text { Field }\end{array}$ & $\begin{array}{c}\text { Primary } \\
\text { Key }\end{array}$ & Definition \\
\hline CreatedBy & varchar & 64 & 0 & $\mathrm{Y}$ & $\mathrm{N}$ & Database username of person who created record. \\
CreatedDate & datetime & 0 & 0 & $\mathrm{Y}$ & $\mathrm{N}$ & Date and time when record was created. \\
EditDate & datetime & 0 & 0 & $\mathrm{~N}$ & $\mathrm{~N}$ & Date and time when record was most recently updated. \\
EditedBy & varchar & 64 & 0 & $\mathrm{~N}$ & $\mathrm{~N}$ & Database username of person who most recently updated record. \\
KeywordID & bigint & 19 & 0 & $\mathrm{Y}$ & $\mathrm{Y}$ & Associated Keywords record identifier. \\
SurveyDescriptionID & int & 0 & 0 & $\mathrm{Y}$ & $\mathrm{Y}$ & Associated SurveyDescription record identifier. \\
\hline
\end{tabular}

\section{StudyPersonnel Table}

\begin{tabular}{lcccccl}
\hline \multicolumn{1}{c}{ Field Name } & $\begin{array}{c}\text { Data } \\
\text { Type }\end{array}$ & $\begin{array}{c}\text { Field } \\
\text { Length }\end{array}$ & $\begin{array}{c}\text { Decimal } \\
\text { Places }\end{array}$ & $\begin{array}{c}\text { Required } \\
\text { Field }\end{array}$ & $\begin{array}{c}\text { Primary } \\
\text { Key }\end{array}$ & Definition \\
\hline AffiliateID & int & 0 & 0 & $\mathrm{Y}$ & $\mathrm{N}$ & Associated Affiliate record identifier. \\
CreatedBy & varchar & 64 & 0 & $\mathrm{Y}$ & $\mathrm{N}$ & Database username of person who created record. \\
CreatedDate & datetime & 0 & 0 & $\mathrm{Y}$ & $\mathrm{N}$ & Date and time when record was created. \\
EditDate & datetime & 0 & 0 & $\mathrm{~N}$ & $\mathrm{~N}$ & Date and time when record was most recently updated. \\
EditedBy & varchar & 64 & 0 & $\mathrm{~N}$ & $\mathrm{~N}$ & Database username of person who most recently updated record. \\
Notes & varchar & 250 & 0 & $\mathrm{~N}$ & $\mathrm{~N}$ & Notes about the study personnel. \\
Role & varchar & 50 & 0 & $\mathrm{~N}$ & $\mathrm{~N}$ & Role of person involved in the study. See luAffiliateRole. \\
SPID & bigint & 19 & 0 & $\mathrm{Y}$ & $\mathrm{Y}$ & Study Personnel record identifier. \\
SurveyDescriptionID & int & 0 & 0 & $\mathrm{Y}$ & $\mathrm{N}$ & Associated SurveyDescription record identifier. \\
\hline
\end{tabular}

\section{SuccessCriteria Table}

\begin{tabular}{|c|c|c|c|c|c|c|}
\hline Field Name & $\begin{array}{l}\text { Data } \\
\text { Type }\end{array}$ & $\begin{array}{l}\text { Field } \\
\text { Length }\end{array}$ & $\begin{array}{l}\text { Decimal } \\
\text { Places }\end{array}$ & $\begin{array}{l}\text { Required } \\
\text { Field }\end{array}$ & $\begin{array}{l}\text { Primary } \\
\text { Key }\end{array}$ & Definition \\
\hline CreatedBy & varchar & 64 & 0 & Y & $\mathrm{N}$ & Database username of person who created record. \\
\hline Description & varchar & 255 & 0 & $\mathrm{~N}$ & $\mathrm{~N}$ & $\begin{array}{l}\text { Describe the general criteria used to determine success of } \\
\text { efforts. }\end{array}$ \\
\hline EditDate & datetime & 0 & 0 & $\mathrm{~N}$ & $\mathrm{~N}$ & Date and time when record was most recently updated. \\
\hline ManagementActivityID & bigint & 19 & 0 & $\mathrm{Y}$ & $\mathrm{N}$ & Associated ManagementActivity record identifier. \\
\hline
\end{tabular}




\begin{tabular}{lcccccl}
\multicolumn{1}{l}{ SuccessCriteria Table-Continued } \\
\multicolumn{1}{c}{ Field Name } & $\begin{array}{c}\text { Data } \\
\text { Type }\end{array}$ & $\begin{array}{c}\text { Field } \\
\text { Length }\end{array}$ & $\begin{array}{c}\text { Decimal } \\
\text { Places }\end{array}$ & $\begin{array}{c}\text { Required } \\
\text { Field }\end{array}$ & $\begin{array}{c}\text { Primary } \\
\text { Key }\end{array}$ & Definition \\
\hline Notes & varchar & 255 & 0 & $\mathrm{~N}$ & $\mathrm{~N}$ & Notes about the Success Criteria. \\
QAQC_Note & varchar & 255 & 0 & $\mathrm{~N}$ & $\mathrm{~N}$ & Reason for change made to record during QAQC review. \\
QAQC_Status & varchar & 20 & 0 & $\mathrm{Y}$ & $\mathrm{N}$ & Describes current stage of QAQC. \\
SCNumber & int & 0 & 0 & $\mathrm{~N}$ & $\mathrm{~N}$ & Number identifier of success criteria \\
SpecificTaxa & char & 6 & 0 & $\mathrm{~N}$ & $\mathrm{~N}$ & TaxaID to which the success criteria pertains. \\
SuccessCriteriaID & bigint & 19 & 0 & $\mathrm{Y}$ & $\mathrm{Y}$ & Success Criteria record identifier.
\end{tabular}

\section{SurveyDescription Table}

\begin{tabular}{|c|c|c|c|c|c|c|}
\hline Field Name & $\begin{array}{l}\text { Data } \\
\text { Type }\end{array}$ & $\begin{array}{l}\text { Field } \\
\text { Length }\end{array}$ & $\begin{array}{l}\text { Decimal } \\
\text { Places }\end{array}$ & $\begin{array}{l}\text { Required } \\
\text { Field }\end{array}$ & $\begin{array}{l}\text { Primary } \\
\text { Key }\end{array}$ & Definition \\
\hline CompletionDate & datetime & 0 & 0 & $\mathrm{~N}$ & $\mathrm{~N}$ & Date surveys were completed. \\
\hline CreatedBy & varchar & 64 & 0 & $\mathrm{Y}$ & $\mathrm{N}$ & Database username of person who created record. \\
\hline CreatedDate & datetime & 0 & 0 & $\mathrm{Y}$ & $\mathrm{N}$ & Date and time when record was created. \\
\hline CurrentStage & varchar & 25 & 0 & $\mathrm{~N}$ & $\mathrm{~N}$ & The current stage of the study. \\
\hline EditedBy & varchar & 64 & 0 & $\mathrm{~N}$ & $\mathrm{~N}$ & $\begin{array}{l}\text { Database username of person who most recently } \\
\text { updated record. }\end{array}$ \\
\hline GeneralLocation & varchar & 50 & 0 & $\mathrm{~N}$ & $\mathrm{~N}$ & $\begin{array}{l}\text { Associated record identifier of site that study was } \\
\text { generally located in. }\end{array}$ \\
\hline HypotheticalQuestionNotes & varchar & 300 & 0 & $\mathrm{~N}$ & $\mathrm{~N}$ & Hypothetical question notes. \\
\hline SourceType & int & 0 & 0 & $\mathrm{~N}$ & $\mathrm{~N}$ & $\begin{array}{l}\text { Distinguish the original source of the data. See } \\
\text { luSourceType. }\end{array}$ \\
\hline StudyName & varchar & 150 & 0 & $\mathrm{~N}$ & $\mathrm{~N}$ & Name of the study. \\
\hline StudyType & varchar & 40 & 0 & $\mathrm{~N}$ & $\mathrm{~N}$ & Type of study conducted. See luStudyType. \\
\hline SurveyDescriptionID & int & 0 & 0 & $\mathrm{Y}$ & $\mathrm{Y}$ & Survey Description record identifier. \\
\hline SurveyDescriptionRecordValidated & bit & 0 & 0 & $\mathrm{~N}$ & $\mathrm{~N}$ & $\mathrm{Y} / \mathrm{N}$. Has this record been validated? \\
\hline SurveyNotes & varchar & 500 & 0 & $\mathrm{~N}$ & $\mathrm{~N}$ & Survey notes. \\
\hline SurveyObjectives & varchar & 500 & 0 & $\mathrm{~N}$ & $\mathrm{~N}$ & Survey objectives. \\
\hline
\end{tabular}

\section{SurveyDescription_EstimateSeries Table}

\begin{tabular}{lcccccl}
\hline Field Name & $\begin{array}{c}\text { Data } \\
\text { Type }\end{array}$ & $\begin{array}{c}\text { Field } \\
\text { Length }\end{array}$ & $\begin{array}{c}\text { Decimal } \\
\text { Places }\end{array}$ & $\begin{array}{c}\text { Required } \\
\text { Field }\end{array}$ & $\begin{array}{c}\text { Primary } \\
\text { Key }\end{array}$ & Definition \\
\hline CreatedBy & varchar & 64 & 0 & $\mathrm{Y}$ & $\mathrm{N}$ & Database username of person who created record. \\
CreatedDate & datetime & 0 & 0 & $\mathrm{Y}$ & $\mathrm{N}$ & Date and time when record was created. \\
EditDate & char & 10 & 0 & $\mathrm{~N}$ & $\mathrm{~N}$ & Date and time when record was most recently updated. \\
EditedBy & varchar & 64 & 0 & $\mathrm{~N}$ & $\mathrm{~N}$ & Database username of person who most recently updated record. \\
ESID & bigint & 19 & 0 & $\mathrm{Y}$ & $\mathrm{Y}$ & Associated EstimateSeries record identifier. \\
SurveyDescriptionID & int & 0 & 0 & $\mathrm{Y}$ & $\mathrm{Y}$ & Associated Survey Description record identifier. \\
\hline
\end{tabular}


SurveyDescription_Project Table

\begin{tabular}{lcccccl}
\hline \multicolumn{1}{c}{ Field Name } & $\begin{array}{c}\text { Data } \\
\text { Type }\end{array}$ & $\begin{array}{c}\text { Field } \\
\text { Length }\end{array}$ & $\begin{array}{c}\text { Decimal } \\
\text { Places }\end{array}$ & $\begin{array}{c}\text { Required } \\
\text { Field }\end{array}$ & $\begin{array}{c}\text { Primary } \\
\text { Key }\end{array}$ & Definition \\
\hline CreatedBy & varchar & 64 & 0 & $\mathrm{Y}$ & $\mathrm{N}$ & Database username of person who created record. \\
CreatedDate & datetime & 0 & 0 & $\mathrm{Y}$ & $\mathrm{N}$ & Date and time when record was created. \\
EditDate & datetime & 0 & 0 & $\mathrm{~N}$ & $\mathrm{~N}$ & Date and time when record was most recently updated. \\
EditedBy & varchar & 64 & 0 & $\mathrm{~N}$ & $\mathrm{~N}$ & Database username of person who most recently updated record. \\
ProjectID & varchar & 12 & 0 & $\mathrm{Y}$ & $\mathrm{Y}$ & ProjectID record identifier. \\
SurveyDescriptionID & int & 0 & 0 & $\mathrm{Y}$ & $\mathrm{Y}$ & Survey Description record identifier. \\
\hline
\end{tabular}

\section{SurveyDescription_Site Table}

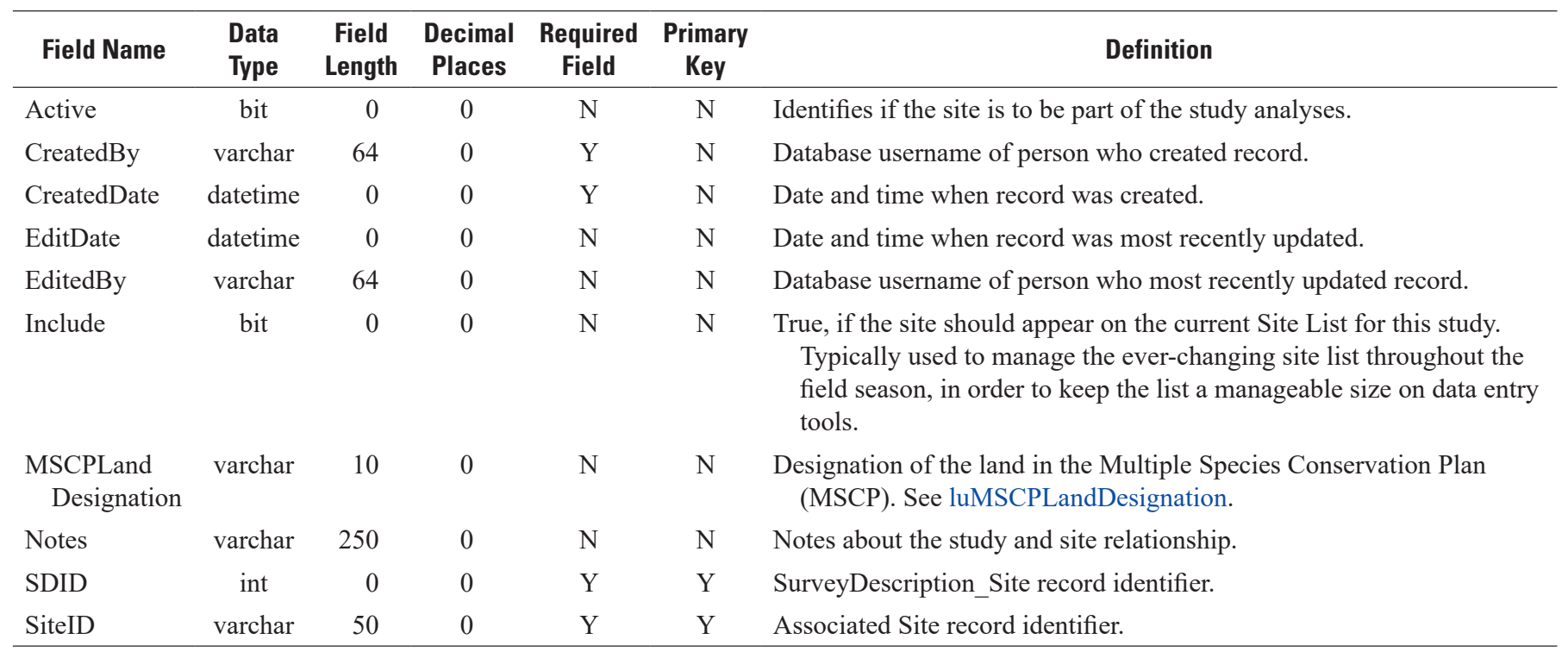

\section{SurveyEvent Table}

\begin{tabular}{|c|c|c|c|c|c|c|}
\hline Field Name & $\begin{array}{l}\text { Data } \\
\text { Type }\end{array}$ & $\begin{array}{c}\text { Field } \\
\text { Length }\end{array}$ & $\begin{array}{l}\text { Decimal } \\
\text { Places }\end{array}$ & $\begin{array}{l}\text { Required } \\
\text { Field }\end{array}$ & $\begin{array}{l}\text { Primary } \\
\text { Key }\end{array}$ & Definition \\
\hline ActionID & bigint & 19 & 0 & $\mathrm{~N}$ & $\mathrm{~N}$ & Associated Action record identifier. \\
\hline ConstrainedBy & char & 4 & 0 & $\mathrm{~N}$ & $\mathrm{~N}$ & $\begin{array}{l}\text { Metric by which survey was constrained (for example, time or } \\
\text { area). See ConstrainBy table. }\end{array}$ \\
\hline Created & datetime & 0 & 0 & $\mathrm{~N}$ & $\mathrm{~N}$ & Pendragon PDA field, for PDA compatibility. \\
\hline CreatedBy & varchar & 64 & 0 & $\mathrm{Y}$ & $\mathrm{N}$ & Database username of person who created record. \\
\hline CreatedDate & datetime & 0 & 0 & $\mathrm{Y}$ & $\mathrm{N}$ & Date and time when record was created. \\
\hline DeviceID & varchar & 25 & 0 & $\mathrm{~N}$ & $\mathrm{~N}$ & $\begin{array}{l}\text { For surveys using some type of automated instrument for } \\
\text { making observations, the unique name or ID of the individual } \\
\text { device. }\end{array}$ \\
\hline EditDate & datetime & 0 & 0 & $\mathrm{~N}$ & $\mathrm{~N}$ & Date and time when record was most recently updated. \\
\hline
\end{tabular}


SurveyEvent Table-Continued

\begin{tabular}{|c|c|c|c|c|c|c|}
\hline Field Name & $\begin{array}{l}\text { Data } \\
\text { Type }\end{array}$ & $\begin{array}{l}\text { Field } \\
\text { Length }\end{array}$ & $\begin{array}{l}\text { Decimal } \\
\text { Places }\end{array}$ & $\begin{array}{l}\text { Required } \\
\text { Field }\end{array}$ & $\begin{array}{l}\text { Primary } \\
\text { Key }\end{array}$ & Definition \\
\hline EditedBy & varchar & 64 & 0 & $\mathrm{~N}$ & $\mathrm{~N}$ & $\begin{array}{l}\text { Database username of person who most recently updated } \\
\text { record. }\end{array}$ \\
\hline EffortValue & decimal & 20 & 5 & $\mathrm{~N}$ & $\mathrm{~N}$ & Numeric value of the effort. \\
\hline EndDateTime & datetime & 0 & 0 & $\mathrm{~N}$ & $\mathrm{~N}$ & Date and time survey ends. \\
\hline HistDataRecordID & varchar & 50 & 0 & $\mathrm{~N}$ & $\mathrm{~N}$ & $\begin{array}{l}\text { The original primary key value, for legacy records that have } \\
\text { been migrated into this database. }\end{array}$ \\
\hline HistDatasetName & varchar & 50 & 0 & $\mathrm{~N}$ & $\mathrm{~N}$ & $\begin{array}{l}\text { The name used to refer to the original dataset, for legacy } \\
\text { records that have been migrated into this database. }\end{array}$ \\
\hline ParentSE & int & 0 & 0 & $\mathrm{~N}$ & $\mathrm{~N}$ & $\begin{array}{l}\text { Parent SurveyEvent record identifier. Used if survey is a } \\
\text { subsurvey. }\end{array}$ \\
\hline QAQC_Note & varchar & 255 & 0 & $\mathrm{~N}$ & $\mathrm{~N}$ & Reason for change made to record during QAQC review. \\
\hline StandSizeObserved & numeric & 10 & 0 & $\mathrm{~N}$ & $\mathrm{~N}$ & Size of the stand observed during survey. \\
\hline StartDateTime & datetime & 0 & 0 & $\mathrm{Y}$ & $\mathrm{N}$ & Date and time survey begins. \\
\hline Subsurvey_conducted & bit & 0 & 0 & $\mathrm{~N}$ & $\mathrm{~N}$ & Whether or not a sub-survey was conducted. \\
\hline SurveyableArea & numeric & 8 & 2 & $\mathrm{~N}$ & $\mathrm{~N}$ & $\begin{array}{l}\text { Area of Site that is surveyable (excluding areas not } \\
\text { surveyabledue to some constraint). }\end{array}$ \\
\hline SurveyableAreaUnit & varchar & 20 & 0 & $\mathrm{~N}$ & $\mathrm{~N}$ & Unit of surveyable area. See luSurveyableAreaUnit. \\
\hline SurveyArea & decimal & 20 & 5 & $\mathrm{~N}$ & $\mathrm{~N}$ & Survey area in square meters. \\
\hline SurveyDescriptionID & int & 0 & 0 & $\mathrm{Y}$ & $\mathrm{N}$ & Associated SurveyDescription record identifier. \\
\hline SurveyEventID & varchar & 50 & 0 & $\mathrm{Y}$ & $\mathrm{Y}$ & SurveyEvent record identifier. \\
\hline Type & varchar & 25 & 0 & $\mathrm{~N}$ & $\mathrm{~N}$ & Type of survey event. See luStudyType. \\
\hline UnitEffortID & char & 2 & 0 & $\mathrm{~N}$ & $\mathrm{~N}$ & Unit in which effort is measured. See UnitEffort table. \\
\hline UnitID & int & 0 & 0 & $\mathrm{~N}$ & $\mathrm{~N}$ & Pendragon PDA field, for PDA compatibility. \\
\hline UserName & varchar & 64 & 0 & $\mathrm{~N}$ & $\mathrm{~N}$ & Pendragon PDA field, for PDA compatibility. \\
\hline
\end{tabular}

SurveyEvent_Affiliate Table

\begin{tabular}{lcccccl}
\hline \multicolumn{1}{c}{ Field Name } & $\begin{array}{c}\text { Data } \\
\text { Type }\end{array}$ & $\begin{array}{c}\text { Field } \\
\text { Length }\end{array}$ & $\begin{array}{c}\text { Decimal } \\
\text { Places }\end{array}$ & $\begin{array}{c}\text { Required } \\
\text { Field }\end{array}$ & $\begin{array}{c}\text { Primary } \\
\text { Key }\end{array}$ & Definition \\
\hline AffiliateID & int & 0 & 0 & $\mathrm{Y}$ & $\mathrm{Y}$ & Associated Affiliate record identifier. \\
Created & datetime & 0 & 0 & $\mathrm{~N}$ & $\mathrm{~N}$ & Pendragon PDA field, for PDA compatibility. \\
CreatedBy & varchar & 64 & 0 & $\mathrm{Y}$ & $\mathrm{N}$ & Database username of person who created record. \\
CreatedDate & datetime & 0 & 0 & $\mathrm{Y}$ & $\mathrm{N}$ & Date and time when record was created. \\
EditDate & datetime & 0 & 0 & $\mathrm{~N}$ & $\mathrm{~N}$ & Date and time when record was most recently updated. \\
EditedBy & varchar & 64 & 0 & $\mathrm{~N}$ & $\mathrm{~N}$ & Database username of person who most recently updated record. \\
ObserverOrder & int & 0 & 0 & $\mathrm{~N}$ & $\mathrm{~N}$ & Order of observers in the survey event. \\
QAQC_Note & varchar & 255 & 0 & $\mathrm{~N}$ & $\mathrm{~N}$ & Reason for change made to record during QAQC review.
\end{tabular}


SurveyEvent_Affiliate Table-Continued

\begin{tabular}{lcccccl}
\hline \multicolumn{1}{c}{ Field Name } & $\begin{array}{c}\text { Data } \\
\text { Type }\end{array}$ & $\begin{array}{c}\text { Field } \\
\text { Length }\end{array}$ & $\begin{array}{c}\text { Decimal } \\
\text { Places }\end{array}$ & $\begin{array}{c}\text { Required } \\
\text { Field }\end{array}$ & $\begin{array}{c}\text { Primary } \\
\text { Key }\end{array}$ & Definition \\
\hline QAQC_Status & varchar & 20 & 0 & $\mathrm{Y}$ & $\mathrm{N}$ & Describes current stage of QAQC. \\
RecordID & int & 0 & 0 & $\mathrm{~N}$ & $\mathrm{~N}$ & Pendragon PDA field, for PDA compatibility. \\
Role & varchar & 50 & 0 & $\mathrm{Y}$ & $\mathrm{Y}$ & Role of person associated with this survey. See luAffiliateRole. \\
SurveyEventID & varchar & 50 & 0 & $\mathrm{Y}$ & $\mathrm{Y}$ & Associated SurveyEvent record identifier. \\
UnitID & int & 0 & 0 & $\mathrm{~N}$ & $\mathrm{~N}$ & Pendragon PDA field, for PDA compatibility. \\
UserName & varchar & 64 & 0 & $\mathrm{~N}$ & $\mathrm{~N}$ & Pendragon PDA field, for PDA compatibility. \\
\hline
\end{tabular}

\section{SurveyEvent_Site Table}

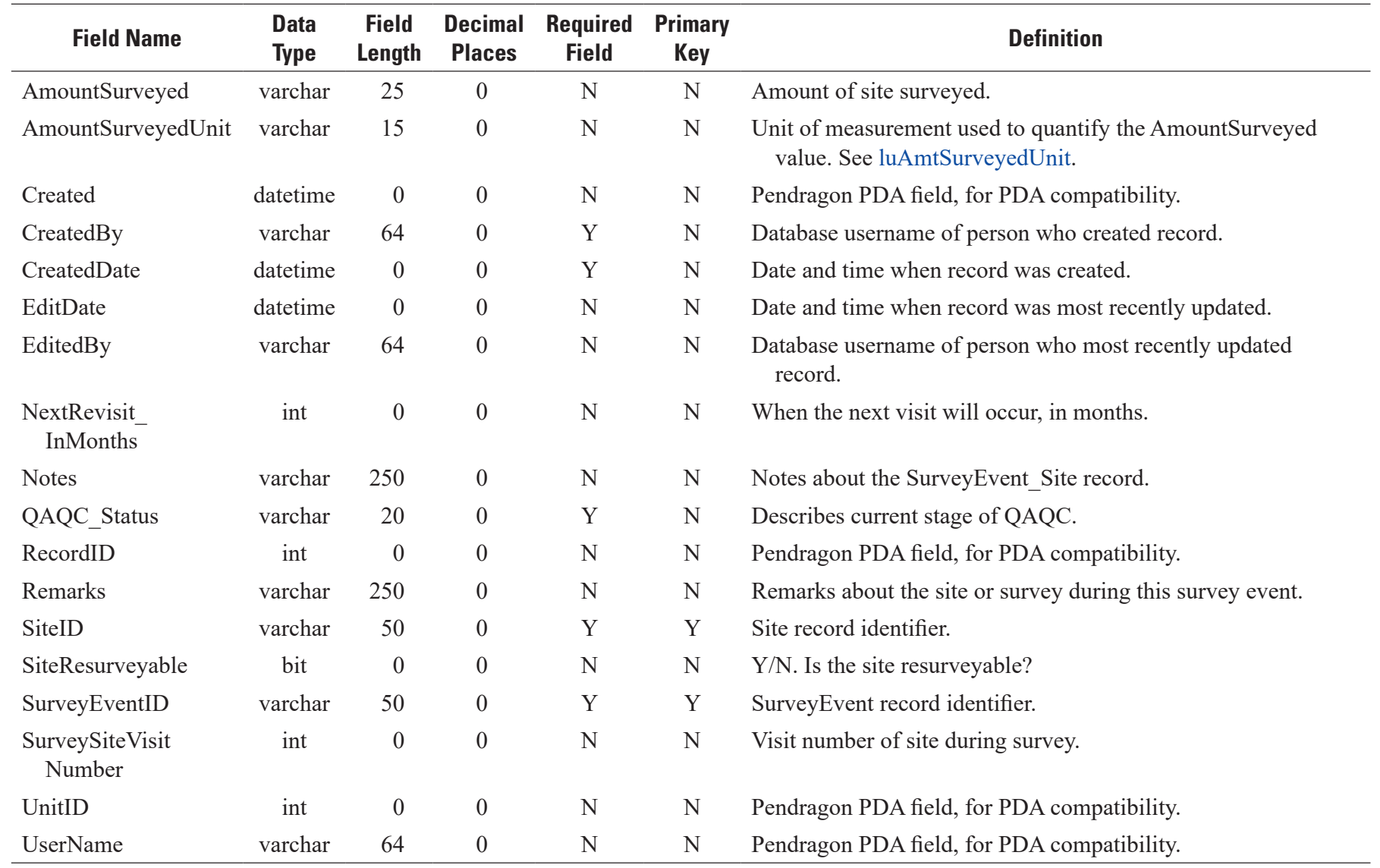

\section{SurveyEvent_SpatialFeature Table}

\begin{tabular}{lcccccl}
\hline \multicolumn{1}{c}{ Field Name } & $\begin{array}{c}\text { Data } \\
\text { Type }\end{array}$ & $\begin{array}{c}\text { Field } \\
\text { Length }\end{array}$ & $\begin{array}{c}\text { Decimal } \\
\text { Places }\end{array}$ & $\begin{array}{c}\text { Required } \\
\text { Field }\end{array}$ & $\begin{array}{c}\text { Primary } \\
\text { Key }\end{array}$ & Definition \\
\hline CreatedBy & varchar & 64 & 0 & $\mathrm{Y}$ & $\mathrm{N}$ & Database username of person who created record. \\
CreatedDate & datetime & 0 & 0 & $\mathrm{Y}$ & $\mathrm{N}$ & Date and time when record was created. \\
EditDate & datetime & 0 & 0 & $\mathrm{~N}$ & $\mathrm{~N}$ & Date and time when record was most recently updated. \\
EditedBy & varchar & 64 & 0 & $\mathrm{~N}$ & $\mathrm{~N}$ & Database username of person who most recently updated record. \\
Notes & varchar & 250 & 0 & $\mathrm{~N}$ & $\mathrm{~N}$ & Notes about the SurveyEvent_SpatialFeature record. \\
Relationship & varchar & 20 & 0 & $\mathrm{~N}$ & $\mathrm{~N}$ & How the survey event record is related to the spatial feature record. \\
& & & & & & See luSESFRelationship. \\
SpatialFeatureID & varchar & 50 & 0 & $\mathrm{Y}$ & $\mathrm{Y}$ & SpatialFeature record identifier. \\
SurveyEventID & varchar & 50 & 0 & $\mathrm{Y}$ & $\mathrm{Y}$ & SurveyEvent record identifier. \\
\hline
\end{tabular}


SurveyMethod Table

\begin{tabular}{lcccccl}
\hline \multicolumn{1}{c}{ Field Name } & $\begin{array}{c}\text { Data } \\
\text { Type }\end{array}$ & $\begin{array}{c}\text { Field } \\
\text { Length }\end{array}$ & $\begin{array}{c}\text { Decimal } \\
\text { Places }\end{array}$ & $\begin{array}{c}\text { Required } \\
\text { Field }\end{array}$ & $\begin{array}{c}\text { Primary } \\
\text { Key }\end{array}$ & Definition \\
\hline CreatedBy & varchar & 64 & 0 & $\mathrm{Y}$ & $\mathrm{N}$ & Database username of person who created record. \\
CreatedDate & datetime & 0 & 0 & $\mathrm{Y}$ & $\mathrm{N}$ & Date and time when record was created. \\
EditDate & datetime & 0 & 0 & $\mathrm{~N}$ & $\mathrm{~N}$ & Date and time when record was most recently updated. \\
EditedBy & varchar & 64 & 0 & $\mathrm{~N}$ & $\mathrm{~N}$ & Database username of person who most recently updated record. \\
MethodOrder & int & 0 & 0 & $\mathrm{Y}$ & $\mathrm{N}$ & Order/Heirarchy of SurveyMethod used during a Survey. \\
SMID & varchar & 50 & 0 & $\mathrm{Y}$ & $\mathrm{Y}$ & SurveyMethod record identifier. \\
SurveyEventID & varchar & 50 & 0 & $\mathrm{Y}$ & $\mathrm{N}$ & Associated SurveyEvent record identifier. \\
SurveyMethod & varchar & 30 & 0 & $\mathrm{Y}$ & $\mathrm{N}$ & Method used to detect a taxon during the survey. See luSurveyMethod.
\end{tabular}

\section{TargetVegCommunities Table}

\begin{tabular}{lcccccl}
\hline \multicolumn{1}{c}{ Field Name } & $\begin{array}{c}\text { Data } \\
\text { Type }\end{array}$ & $\begin{array}{c}\text { Field } \\
\text { Length }\end{array}$ & $\begin{array}{c}\text { Decimal } \\
\text { Places }\end{array}$ & $\begin{array}{c}\text { Required } \\
\text { Field }\end{array}$ & $\begin{array}{c}\text { Primary } \\
\text { Key }\end{array}$ & Definition \\
\hline CreatedBy & varchar & 64 & 0 & $\mathrm{Y}$ & $\mathrm{N}$ & Database username of person who created record. \\
CreatedDate & datetime & 0 & 0 & $\mathrm{Y}$ & $\mathrm{N}$ & Date and time when record was created. \\
EditDate & datetime & 0 & 0 & $\mathrm{~N}$ & $\mathrm{~N}$ & Date and time when record was most recently updated. \\
EditedBy & varchar & 64 & 0 & $\mathrm{~N}$ & $\mathrm{~N}$ & Database username of person who most recently updated record. \\
Notes & varchar & 250 & 0 & $\mathrm{~N}$ & $\mathrm{~N}$ & Notes about the target vegetation community record. \\
QAQC_Notes & varchar & 255 & 0 & $\mathrm{~N}$ & $\mathrm{~N}$ & Reason for change made to record during QAQC review. \\
QAQC_Status & varchar & 20 & 0 & $\mathrm{Y}$ & $\mathrm{N}$ & Describes current stage of QAQC. \\
SurveyDescriptionID & int & 0 & 0 & $\mathrm{Y}$ & $\mathrm{N}$ & Associated SurveyDescription record identifier. \\
TaxaID & varchar & 25 & 0 & $\mathrm{Y}$ & $\mathrm{N}$ & Taxonomic Serial Number (www.itis.gov) of Target Vegetation \\
& & & & & & Community. \\
TVCID & bigint & 19 & 0 & $\mathrm{Y}$ & $\mathrm{Y}$ & TargetVegCommunites record identifier. \\
\hline
\end{tabular}

\section{TaxaObservation Table}

\begin{tabular}{|c|c|c|c|c|c|c|}
\hline Field Name & $\begin{array}{l}\text { Data } \\
\text { Type }\end{array}$ & $\begin{array}{l}\text { Field } \\
\text { Length }\end{array}$ & $\begin{array}{l}\text { Decimal } \\
\text { Places }\end{array}$ & $\begin{array}{l}\text { Required } \\
\text { Field }\end{array}$ & $\begin{array}{l}\text { Primary } \\
\text { Key }\end{array}$ & Definition \\
\hline $\begin{array}{l}\text { AbundanceCategory } \\
\text { Code }\end{array}$ & varchar & 2 & 0 & $\mathrm{~N}$ & $\mathrm{~N}$ & $\begin{array}{l}\text { How abundant the individuals of this species were found to be } \\
\text { during this observation. See luAbundanceCategory table. }\end{array}$ \\
\hline AerialExtent & int & 0 & 0 & $\mathrm{~N}$ & $\mathrm{~N}$ & $\begin{array}{l}\text { Extent of area of taxon in square meters (only applies to } \\
\text { vegetation). }\end{array}$ \\
\hline Created & datetime & 0 & 0 & $\mathrm{~N}$ & $\mathrm{~N}$ & Pendragon PDA field, for PDA compatibility. \\
\hline CreatedDate & datetime & 0 & 0 & $\mathrm{Y}$ & $\mathrm{N}$ & Date and time when record was created. \\
\hline EditDate & datetime & 0 & 0 & $\mathrm{~N}$ & $\mathrm{~N}$ & Date and time when record was most recently updated. \\
\hline EditedBy & varchar & 64 & 0 & $\mathrm{~N}$ & $\mathrm{~N}$ & Database username of person who most recently updated record. \\
\hline HistDataRecordID & varchar & 50 & 0 & $\mathrm{~N}$ & $\mathrm{~N}$ & For migrated records, primary key value from original source. \\
\hline HistDataSetName & varchar & 50 & 0 & $\mathrm{~N}$ & $\mathrm{~N}$ & For migrated records, name of dataset from legacy source. \\
\hline IdentifiedByAffiliate & int & 0 & 0 & $\mathrm{~N}$ & $\mathrm{~N}$ & $\begin{array}{l}\text { Affiliate who identifies or confirms identification of the animal or } \\
\text { plant, when different from observer. }\end{array}$ \\
\hline IdentifiedByDate & datetime & 0 & 0 & $\mathrm{~N}$ & $\mathrm{~N}$ & Date plant or animal was identified. \\
\hline
\end{tabular}


TaxaObservation Table-Continued

\begin{tabular}{|c|c|c|c|c|c|c|}
\hline Field Name & $\begin{array}{l}\text { Data } \\
\text { Type }\end{array}$ & $\begin{array}{l}\text { Field } \\
\text { Length }\end{array}$ & $\begin{array}{l}\text { Decimal } \\
\text { Places }\end{array}$ & $\begin{array}{l}\text { Required } \\
\text { Field }\end{array}$ & $\begin{array}{l}\text { Primary } \\
\text { Key }\end{array}$ & Definition \\
\hline Notes & varchar & 255 & 0 & $\mathrm{~N}$ & $\mathrm{~N}$ & Notes about the observation. \\
\hline PercentExtent & int & 0 & 0 & $\mathrm{~N}$ & $\mathrm{~N}$ & Percent extent of taxon observation (only applies to vegetation). \\
\hline QAQC_Note & varchar & 255 & 0 & $\mathrm{~N}$ & $\mathrm{~N}$ & Reason for change made to record during QAQC review. \\
\hline Remarks & varchar & 250 & 0 & $\mathrm{~N}$ & $\mathrm{~N}$ & Remarks about this observation. \\
\hline $\begin{array}{l}\text { RemovalIntro } \\
\text { ValueID }\end{array}$ & int & 0 & 0 & $\mathrm{~N}$ & $\mathrm{~N}$ & $\begin{array}{l}\text { Taxon removal or introduction value. See } \\
\text { luRemovalIntroductionValues. }\end{array}$ \\
\hline SiblingID & varchar & 50 & 0 & $\mathrm{~N}$ & $\mathrm{~N}$ & $\begin{array}{l}\text { Associated observation record identifier. Could be an associated } \\
\text { weather or habitat record, etc. }\end{array}$ \\
\hline SurveyEventID & varchar & 50 & 0 & Y & $\mathrm{N}$ & Associated SurveyEvent record identifier. \\
\hline TaxaID & varchar & 25 & 0 & $\mathrm{~N}$ & $\mathrm{~N}$ & $\begin{array}{l}\text { Taxonomic Serial Number (www.itis.gov) of species. See Taxa } \\
\text { table. }\end{array}$ \\
\hline TaxaObservationID & varchar & 50 & 0 & $\mathrm{Y}$ & $\mathrm{Y}$ & TaxaObservation record identifier. \\
\hline TaxaRoleID & int & 0 & 0 & $\mathrm{~N}$ & $\mathrm{~N}$ & $\begin{array}{l}\text { Role that this taxon plays regarding the survey (e.g. target } \\
\text { species, incidental observation). See luTaxaRole. }\end{array}$ \\
\hline UnitID & int & 0 & 0 & $\mathrm{~N}$ & $\mathrm{~N}$ & Pendragon PDA field, for PDA compatibility. \\
\hline UserName & varchar & 64 & 0 & $\mathrm{~N}$ & $\mathrm{~N}$ & Pendragon PDA field, for PDA compatibility. \\
\hline
\end{tabular}

\section{TaxaObservationDetails Table}

\begin{tabular}{|c|c|c|c|c|c|c|}
\hline Field Name & $\begin{array}{l}\text { Data } \\
\text { Type }\end{array}$ & $\begin{array}{l}\text { Field } \\
\text { Length }\end{array}$ & $\begin{array}{l}\text { Decimal } \\
\text { Places }\end{array}$ & $\begin{array}{l}\text { Required } \\
\text { Field }\end{array}$ & $\begin{array}{l}\text { Primary } \\
\text { Key }\end{array}$ & Definition \\
\hline CountMethodID & int & 0 & 0 & $\mathrm{~N}$ & $\mathrm{~N}$ & How the taxon was counted. See luCountMethod table. \\
\hline CreatedBy & varchar & 64 & 0 & $\mathrm{Y}$ & $\mathrm{N}$ & Database username of person who created record. \\
\hline CreatedDate & datetime & 0 & 0 & $\mathrm{Y}$ & $\mathrm{N}$ & Date and time when record was created. \\
\hline DetectionMethodID & int & 0 & 0 & $\mathrm{~N}$ & $\mathrm{~N}$ & How the observed taxon was detected. See luDetectionMethod. \\
\hline EditDate & datetime & 0 & 0 & $\mathrm{~N}$ & $\mathrm{~N}$ & Date and time when record was most recently updated. \\
\hline EditedBy & varchar & 64 & 0 & $\mathrm{~N}$ & $\mathrm{~N}$ & $\begin{array}{l}\text { Database username of person who most recently updated } \\
\text { record. }\end{array}$ \\
\hline GenderID & int & 0 & 0 & $\mathrm{~N}$ & $\mathrm{~N}$ & Gender of taxon. See luGender table. \\
\hline LocationWithinSite & varchar & 25 & 0 & $\mathrm{~N}$ & $\mathrm{~N}$ & $\begin{array}{l}\text { Descriptive category of portion of site where observation } \\
\text { occurred, i.e., subjectively defined zone, or generalized } \\
\text { habitat category. Used to distinguish portions of a site from } \\
\text { one another with various criteria; can be used as a covariate } \\
\text { in occupancy. }\end{array}$ \\
\hline Notes & varchar & 250 & 0 & $\mathrm{~N}$ & $\mathrm{~N}$ & Notes about the observation. \\
\hline
\end{tabular}


TaxaObservationDetails Table-Continued

\begin{tabular}{|c|c|c|c|c|c|c|}
\hline Field Name & $\begin{array}{l}\text { Data } \\
\text { Type }\end{array}$ & $\begin{array}{l}\text { Field } \\
\text { Length }\end{array}$ & $\begin{array}{l}\text { Decimal } \\
\text { Places }\end{array}$ & $\begin{array}{l}\text { Required } \\
\text { Field }\end{array}$ & $\begin{array}{l}\text { Primary } \\
\text { Key }\end{array}$ & Definition \\
\hline $\begin{array}{l}\text { NumberofIndividuals_ } \\
\text { Exact }\end{array}$ & int & 0 & 0 & $\mathrm{~N}$ & $\mathrm{~N}$ & The exact number of individuals observed. \\
\hline $\begin{array}{l}\text { NumberofIndividuals_ } \\
\text { HighEst }\end{array}$ & int & 0 & 0 & $\mathrm{~N}$ & $\mathrm{~N}$ & The upper bound of estimated number of individuals observed. \\
\hline $\begin{array}{l}\text { NumberofIndividuals } \\
\text { LowEst }\end{array}$ & int & 0 & 0 & $\mathrm{~N}$ & $\mathrm{~N}$ & The lower bound of estimated number of individuals observed. \\
\hline ObservationDate Time & datetime & 0 & 0 & $\mathrm{~N}$ & $\mathrm{~N}$ & Date and time of observation. \\
\hline PointID & varchar & 50 & 0 & $\mathrm{~N}$ & $\mathrm{~N}$ & SpatialFeature record identifier. \\
\hline QAQC_Note & varchar & 255 & 0 & $\mathrm{~N}$ & $\mathrm{~N}$ & Reason for change made to record during QAQC review. \\
\hline QAQC_Status & varchar & 20 & 0 & Y & $\mathrm{N}$ & Describes current stage of QAQC. \\
\hline RecordID & int & 0 & 0 & $\mathrm{~N}$ & $\mathrm{~N}$ & Pendragon PDA field, for PDA compatibility. \\
\hline $\begin{array}{l}\text { TOSpecHabitat } \\
\text { FeatureTypeID }\end{array}$ & int & 0 & 0 & $\mathrm{~N}$ & $\mathrm{~N}$ & $\begin{array}{l}\text { Taxa observation specific habitat feature type. See } \\
\text { luHabitatFeatureType. }\end{array}$ \\
\hline TrapID & varchar & 15 & 0 & $\mathrm{~N}$ & $\mathrm{~N}$ & Trap identifier. \\
\hline TrapType & varchar & 35 & 0 & $\mathrm{~N}$ & $\mathrm{~N}$ & Type of trap used to capture taxon. See luTrapType table. \\
\hline UnitID & int & 0 & 0 & $\mathrm{~N}$ & $\mathrm{~N}$ & Pendragon PDA field, for PDA compatibility. \\
\hline UserName & varchar & 64 & 0 & $\mathrm{~N}$ & $\mathrm{~N}$ & Pendragon PDA field, for PDA compatibility. \\
\hline
\end{tabular}

\section{TelemetryDetails Table}

\begin{tabular}{|c|c|c|c|c|c|c|}
\hline Field Name & $\begin{array}{l}\text { Data } \\
\text { Type }\end{array}$ & $\begin{array}{c}\text { Field } \\
\text { Length }\end{array}$ & $\begin{array}{l}\text { Decimal } \\
\text { Places }\end{array}$ & $\begin{array}{l}\text { Required } \\
\text { Field }\end{array}$ & $\begin{array}{l}\text { Primary } \\
\text { Key }\end{array}$ & Definition \\
\hline Altitude & decimal & 7 & 2 & $\mathrm{~N}$ & $\mathrm{~N}$ & Argos ptt reference altitude (DIAG). \\
\hline APFreq & decimal & 8 & 2 & $\mathrm{~N}$ & $\mathrm{~N}$ & Interpolated freq at Doppler inflection (DIAG). \\
\hline AzFrmDply & decimal & 14 & 10 & $\mathrm{~N}$ & $\mathrm{~N}$ & $\begin{array}{l}\text { Great-circle compass bering (degrees) from the deployment location; } \\
\text { as reported by device. }\end{array}$ \\
\hline BothTest & varchar & 30 & 0 & $\mathrm{~N}$ & $\mathrm{~N}$ & Which 3 directionality tests passed in 'Best Hybrid'. \\
\hline CreatedBy & varchar & 64 & 0 & Y & $\mathrm{N}$ & Database username of person who created record. \\
\hline CreatedDate & datetime & 0 & 0 & $\mathrm{Y}$ & $\mathrm{N}$ & Date and time when record was created. \\
\hline DaysDply & numeric & 4 & 0 & $\mathrm{~N}$ & $\mathrm{~N}$ & $\begin{array}{l}\text { Number of elapsed days since deployment date; as reported by } \\
\text { device. }\end{array}$ \\
\hline Distance & decimal & 6 & 2 & $\mathrm{~N}$ & $\mathrm{~N}$ & Great-circle distance $(\mathrm{km})$ from previous location. \\
\hline Dur & int & 0 & 0 & $\mathrm{~N}$ & $\mathrm{~N}$ & Duration (sec) between first and last message (DIAG). \\
\hline Duty & int & 0 & 0 & $\mathrm{~N}$ & $\mathrm{~N}$ & Unique number for all records within a duty cycle. \\
\hline DutyLocs & numeric & 3 & 0 & $\mathrm{~N}$ & $\mathrm{~N}$ & Total number of passes/locations within duty cycle. \\
\hline EditDate & datetime & 0 & 0 & $\mathrm{~N}$ & $\mathrm{~N}$ & Date and time when record was most recently updated. \\
\hline EditedBy & varchar & 64 & 0 & $\mathrm{~N}$ & $\mathrm{~N}$ & Database username of person who most recently updated record. \\
\hline First & bit & 0 & 0 & $\mathrm{~N}$ & $\mathrm{~N}$ & Flag for first data record for a given animal; as reported by device. \\
\hline
\end{tabular}


TelemetryDetails Table-Continued

\begin{tabular}{|c|c|c|c|c|c|c|}
\hline Field Name & $\begin{array}{l}\text { Data } \\
\text { Type }\end{array}$ & $\begin{array}{l}\text { Field } \\
\text { Length }\end{array}$ & $\begin{array}{l}\text { Decimal } \\
\text { Places }\end{array}$ & $\begin{array}{l}\text { Required } \\
\text { Field }\end{array}$ & $\begin{array}{l}\text { Primary } \\
\text { Key }\end{array}$ & Definition \\
\hline GT120 & numeric & 2 & 0 & $\mathrm{~N}$ & $\mathrm{~N}$ & Number of messages with signal strength $<120 \mathrm{~dB}$ (DIAG). \\
\hline iqy & decimal & 3 & 1 & $\mathrm{~N}$ & $\mathrm{~N}$ & $\begin{array}{l}\text { Indicates transmitter oscillator frequency drift between the two } \\
\text { satellite passes. }\end{array}$ \\
\hline Latest & bit & 0 & 0 & $\mathrm{~N}$ & $\mathrm{~N}$ & Flag for most recent data record for an animal; as reported by device. \\
\hline NLoCrank & decimal & 9 & 3 & $\mathrm{~N}$ & $\mathrm{~N}$ & Weighting for best LC94 within duty cycle. \\
\hline NMess & numeric & 3 & 0 & $\mathrm{~N}$ & $\mathrm{~N}$ & Number of messages received during overpass (DIAG). \\
\hline $\mathrm{NoPC}$ & numeric & 1 & 0 & $\mathrm{~N}$ & $\mathrm{~N}$ & How many location plausibility checks $(0-4)$ gave a positive result. \\
\hline SampSize & numeric & 5 & 0 & $\mathrm{~N}$ & $\mathrm{~N}$ & Total number of records for respective animal. \\
\hline Sen 1 & numeric & 3 & 0 & $\mathrm{~N}$ & $\mathrm{~N}$ & Internal temperature of the unit, 8 bits, values between $0-255$. \\
\hline $\operatorname{Sen} 2$ & numeric & 3 & 0 & $\mathrm{~N}$ & $\mathrm{~N}$ & Battery voltage, 8 bits, values between $0-255$. \\
\hline Sen 3 & numeric & 3 & 0 & $\mathrm{~N}$ & $\mathrm{~N}$ & A counter that increments by one on each transmission. \\
\hline $\operatorname{Sen} 4$ & numeric & 3 & 0 & $\mathrm{~N}$ & $\mathrm{~N}$ & Activity. The higher the number, the greater the activity. \\
\hline SpatialFeatureID & varchar & 50 & 0 & $\mathrm{Y}$ & Y & $\begin{array}{l}\text { Associated SpatialFeature record identifier. Referred to as PointID in } \\
\text { some tables. }\end{array}$ \\
\hline Switch & numeric & 1 & 0 & $\mathrm{~N}$ & $\mathrm{~N}$ & Location Choice: $0=$ primary $1=$ alternate $3=$ primary $4=$ alternate. \\
\hline WeedBoth & bit & 0 & 0 & $\mathrm{~N}$ & $\mathrm{~N}$ & 1/0 flag $1=$ reject amending DAR loc with MRD anchors. \\
\hline
\end{tabular}

\section{TerrestrialHabitat Table}

\begin{tabular}{lcccccc}
\hline \multicolumn{1}{c}{ Field Name } & $\begin{array}{c}\text { Data } \\
\text { Type }\end{array}$ & $\begin{array}{c}\text { Field } \\
\text { Length }\end{array}$ & $\begin{array}{c}\text { Decimal } \\
\text { Places }\end{array}$ & $\begin{array}{c}\text { Required } \\
\text { Field }\end{array}$ & $\begin{array}{c}\text { Primary } \\
\text { Key }\end{array}$ & Definition \\
\hline AnnualGrassPercent & int & 0 & 0 & $\mathrm{~N}$ & $\mathrm{~N}$ & Percent of annual grass present in habitat. \\
ConiferHeight & int & 0 & 0 & $\mathrm{~N}$ & $\mathrm{~N}$ & Height of coniferous trees in habitat. \\
ConiferPercent & int & 0 & 0 & $\mathrm{~N}$ & $\mathrm{~N}$ & Percent of coniferous trees present in habitat. \\
Created & datetime & 0 & 0 & $\mathrm{~N}$ & $\mathrm{~N}$ & Pendragon PDA field, for PDA compatibility. \\
CreatedBy & varchar & 64 & 0 & $\mathrm{Y}$ & $\mathrm{N}$ & Database username of person who created record. \\
CreatedDate & datetime & 0 & 0 & $\mathrm{Y}$ & $\mathrm{N}$ & Date and time when record was created. \\
DrySoilPercent & int & 0 & 0 & $\mathrm{~N}$ & $\mathrm{~N}$ & Percent of soil that is dry in habitat. \\
EditDate & datetime & 0 & 0 & $\mathrm{~N}$ & $\mathrm{~N}$ & Date and time when record was most recently updated. \\
EditedBy & varchar & 64 & 0 & $\mathrm{~N}$ & $\mathrm{~N}$ & Database username of person who most recently \\
& & & & & & updated record. \\
ForbPercent & int & 0 & 0 & $\mathrm{~N}$ & $\mathrm{~N}$ & Percent of forbs present in habitat. \\
GrassPercent & int & 0 & 0 & $\mathrm{~N}$ & $\mathrm{~N}$ & Percent of grass present in habitat. \\
GroundPhenology & varchar & 10 & 0 & $\mathrm{~N}$ & $\mathrm{~N}$ & Seasonal timing of biological status of ground cover in \\
& & & & & & habitat. See luPhenology table.
\end{tabular}


TerrestrialHabitat Table-Continued

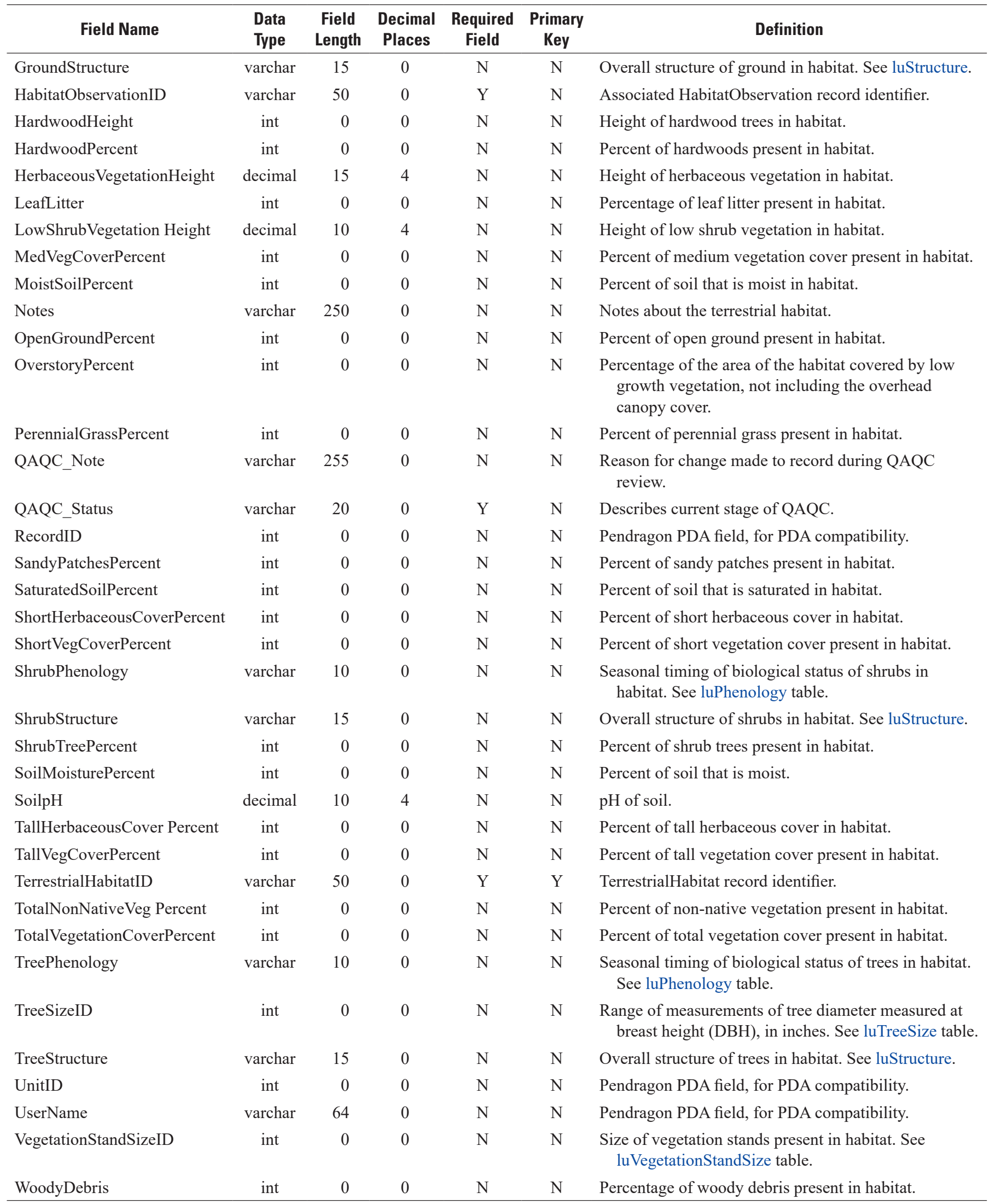


Traps Table

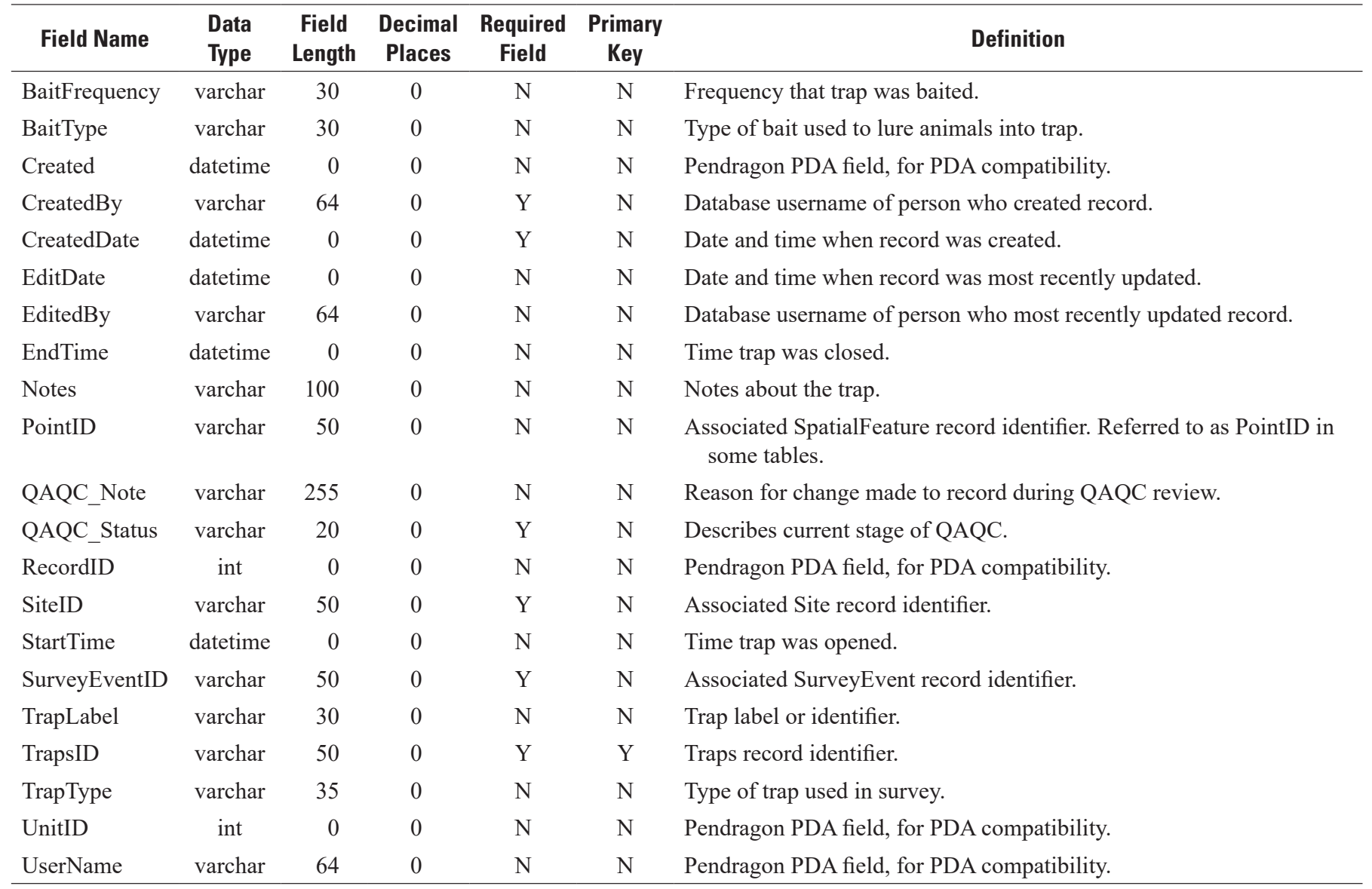

Trauma Table

\begin{tabular}{lcccccl}
\hline \multicolumn{1}{c}{ Field Name } & $\begin{array}{c}\text { Data } \\
\text { Type }\end{array}$ & $\begin{array}{c}\text { Field } \\
\text { Length }\end{array}$ & $\begin{array}{c}\text { Decimal } \\
\text { Places }\end{array}$ & $\begin{array}{c}\text { Required } \\
\text { Field }\end{array}$ & $\begin{array}{c}\text { Primary } \\
\text { Key }\end{array}$ & Definition \\
\hline CreatedBy & varchar & 64 & 0 & $\mathrm{Y}$ & $\mathrm{N}$ & Database username of person who created record. \\
CreatedDate & datetime & 0 & 0 & $\mathrm{Y}$ & $\mathrm{N}$ & Date and time when record was created. \\
EditDate & datetime & 0 & 0 & $\mathrm{~N}$ & $\mathrm{~N}$ & Date and time when record was most recently updated. \\
EditedBy & varchar & 64 & 0 & $\mathrm{~N}$ & $\mathrm{~N}$ & Database username of person who most recently updated record. \\
IAID & varchar & 50 & 0 & $\mathrm{~N}$ & $\mathrm{~N}$ & Associated IndivAnimal record identifier. \\
Notes & varchar & 250 & 0 & $\mathrm{~N}$ & $\mathrm{~N}$ & Notes about the trauma observation. \\
TraumaID & varchar & 50 & 0 & $\mathrm{Y}$ & $\mathrm{Y}$ & Trauma record identifier. \\
TraumaLocation & varchar & 50 & 0 & $\mathrm{~N}$ & $\mathrm{~N}$ & Location of trauma upon subject. \\
TraumaType & varchar & 50 & 0 & $\mathrm{~N}$ & $\mathrm{~N}$ & Type of trauma observed. \\
\hline
\end{tabular}

\section{WaterPhysicalObservation Table}

\begin{tabular}{lcccccl}
\hline \multicolumn{1}{c}{ Field Name } & $\begin{array}{c}\text { Data } \\
\text { Type }\end{array}$ & $\begin{array}{c}\text { Field } \\
\text { Length }\end{array}$ & $\begin{array}{c}\text { Decimal } \\
\text { Places }\end{array}$ & $\begin{array}{c}\text { Required } \\
\text { Field }\end{array}$ & $\begin{array}{c}\text { Primary } \\
\text { Key }\end{array}$ & Definition \\
\hline Created & datetime & 0 & 0 & $\mathrm{~N}$ & $\mathrm{~N}$ & Pendragon PDA field, for PDA compatibility. \\
CreatedBy & varchar & 64 & 0 & $\mathrm{Y}$ & $\mathrm{N}$ & Database username of person who created record. \\
CreatedDate & datetime & 0 & 0 & $\mathrm{Y}$ & $\mathrm{N}$ & Date and time when record was created. \\
Discharge & decimal & 6 & 2 & $\mathrm{~N}$ & $\mathrm{~N}$ & Rate of discharge in cubic feet per second.
\end{tabular}


WaterPhysicalObservation Table-Continued

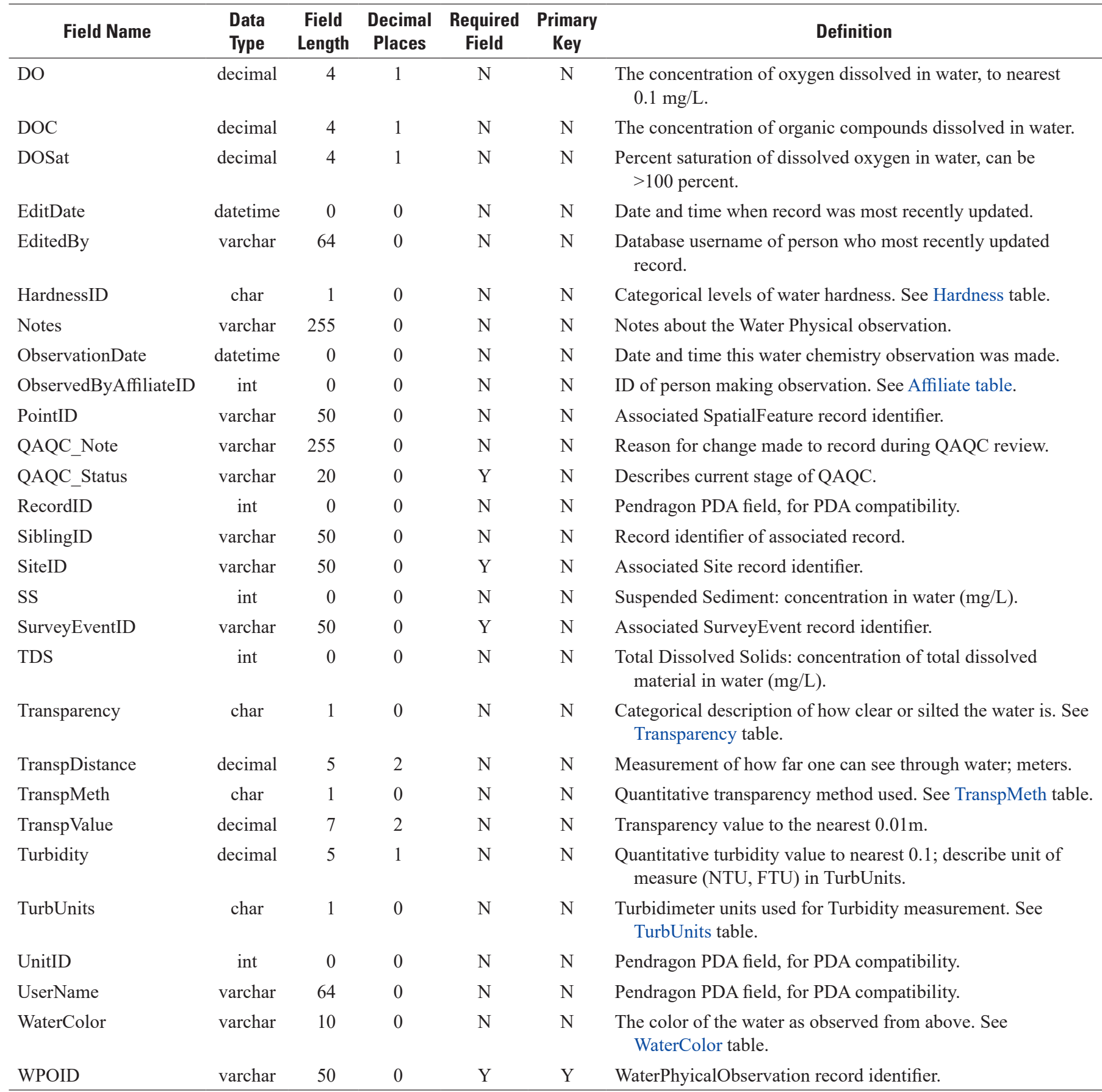

WeatherObservation Table

\begin{tabular}{lcccccl}
\hline \multicolumn{1}{c}{ Field Name } & $\begin{array}{c}\text { Data } \\
\text { Type }\end{array}$ & $\begin{array}{c}\text { Field } \\
\text { Length }\end{array}$ & $\begin{array}{c}\text { Decimal } \\
\text { Places }\end{array}$ & $\begin{array}{c}\text { Required } \\
\text { Field }\end{array}$ & $\begin{array}{c}\text { Primary } \\
\text { Key }\end{array}$ & Definition \\
\hline AirTemp & decimal & 5 & 1 & $\mathrm{~N}$ & $\mathrm{~N}$ & Air temperature taken to nearest 0.1 degree Celsius. \\
BarometerTrend & varchar & 10 & 0 & $\mathrm{~N}$ & $\mathrm{~N}$ & Direction in which barometric readings are going. \\
BarPressure & int & 0 & 0 & $\mathrm{~N}$ & $\mathrm{~N}$ & Barometric pressure (mm Hg). \\
CloudCoverPct & int & 0 & 0 & $\mathrm{~N}$ & $\mathrm{~N}$ & Percent of sky covered with clouds, (0-100). \\
Created & datetime & 0 & 0 & $\mathrm{~N}$ & $\mathrm{~N}$ & Pendragon PDA field, for PDA compatibility.
\end{tabular}


WeatherObservation Table-Continued

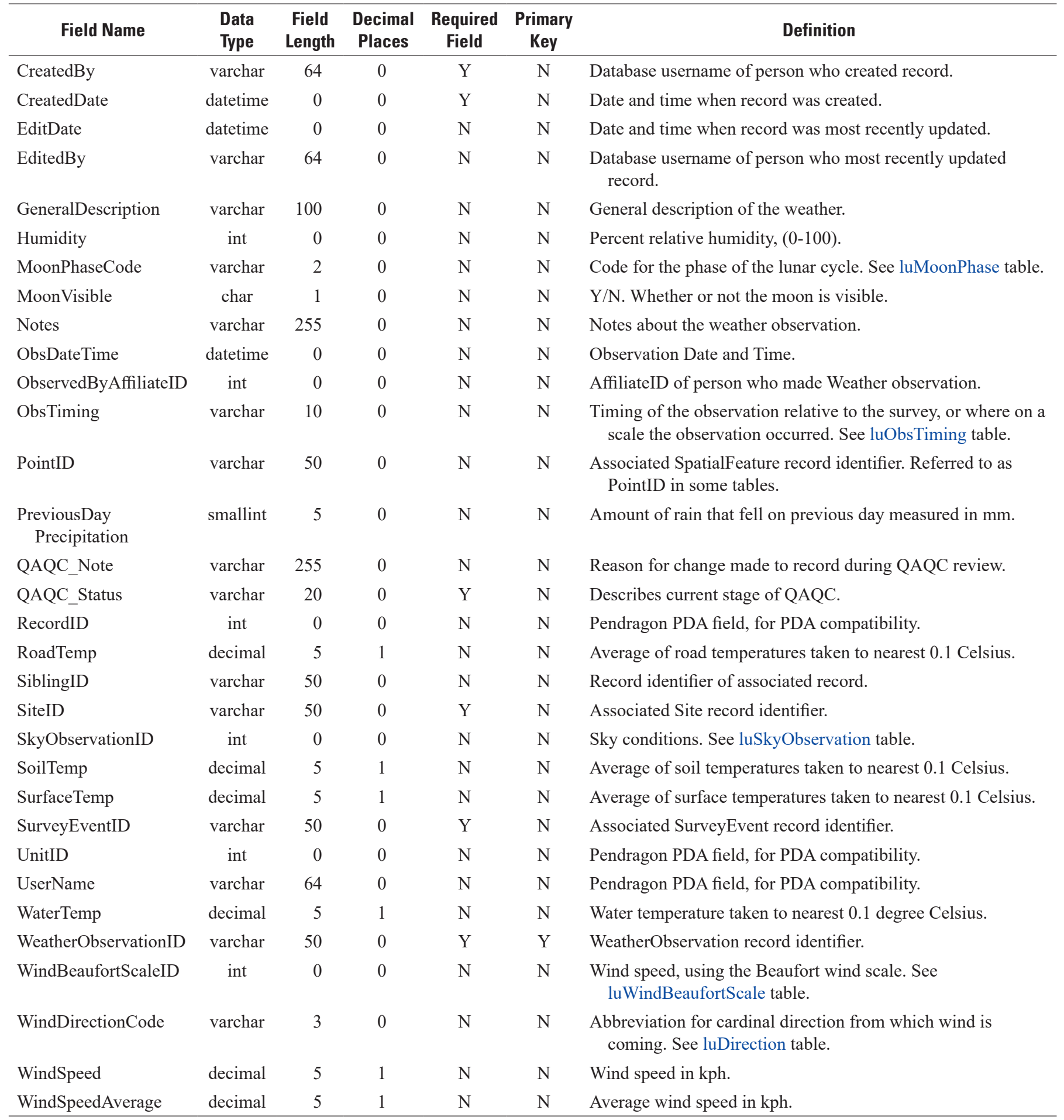

WithinSurveyEffortLost Table

\begin{tabular}{lcccccl}
\hline Field Name & $\begin{array}{c}\text { Data } \\
\text { Type }\end{array}$ & $\begin{array}{c}\text { Field } \\
\text { Length }\end{array}$ & $\begin{array}{c}\text { Decimal } \\
\text { Places }\end{array}$ & $\begin{array}{c}\text { Required } \\
\text { Field }\end{array}$ & $\begin{array}{c}\text { Primary } \\
\text { Key }\end{array}$ & Definition \\
\hline Created & datetime & 0 & 0 & $\mathrm{~N}$ & $\mathrm{~N}$ & Pendragon PDA field, for PDA compatibility. \\
CreatedBy & varchar & 64 & 0 & $\mathrm{Y}$ & $\mathrm{N}$ & Database username of person who created record. \\
CreatedDate & datetime & 0 & 0 & $\mathrm{Y}$ & $\mathrm{N}$ & Date and time when record was created.
\end{tabular}


WithinSurveyEffortLost Table-Continued

\begin{tabular}{|c|c|c|c|c|c|c|}
\hline Field Name & $\begin{array}{l}\text { Data } \\
\text { Type }\end{array}$ & $\begin{array}{c}\text { Field } \\
\text { Length }\end{array}$ & $\begin{array}{l}\text { Decimal } \\
\text { Places }\end{array}$ & $\begin{array}{l}\text { Required } \\
\text { Field }\end{array}$ & $\begin{array}{l}\text { Primary } \\
\text { Key }\end{array}$ & Definition \\
\hline DateClosed & datetime & 0 & 0 & $\mathrm{~N}$ & $\mathrm{~N}$ & Date the trap or array was closed. \\
\hline DisturbanceID & varchar & 50 & 0 & $\mathrm{~N}$ & $\mathrm{~N}$ & $\begin{array}{l}\text { Associated disturbance record identifier of disturbance that } \\
\text { caused closure. }\end{array}$ \\
\hline EditDate & datetime & 0 & 0 & $\mathrm{~N}$ & $\mathrm{~N}$ & Date and time when record was most recently updated. \\
\hline EffortLost & varchar & 75 & 0 & $\mathrm{~N}$ & $\mathrm{~N}$ & $\begin{array}{l}\text { The amount of survey effort lost due to the disturbance to } \\
\text { the trap or array. }\end{array}$ \\
\hline Notes & varchar & 250 & 0 & $\mathrm{~N}$ & $\mathrm{~N}$ & Notes about the survey efffort loss record. \\
\hline RecordID & int & 0 & 0 & $\mathrm{~N}$ & $\mathrm{~N}$ & Pendragon PDA field, for PDA compatibility. \\
\hline SurveyEventID & varchar & 50 & 0 & $\mathrm{Y}$ & $\mathrm{N}$ & Associated SurveyEvent record identifier. \\
\hline UnitID & int & 0 & 0 & $\mathrm{~N}$ & $\mathrm{~N}$ & Pendragon PDA field, for PDA compatibility. \\
\hline UserName & varchar & 64 & 0 & $\mathrm{~N}$ & $\mathrm{~N}$ & Pendragon PDA field, for PDA compatibility. \\
\hline WithinSurveyEffortLostID & varchar & 50 & 0 & $\mathrm{Y}$ & $\mathrm{Y}$ & WithinSurveyEffortLost record identifier. \\
\hline
\end{tabular}

PART 2. LOOK UP TABLES

Actions Table Look Ups

luCHWRHabitat

\begin{tabular}{|c|c|c|c|c|c|c|}
\hline Field Name & $\begin{array}{l}\text { Data } \\
\text { Type }\end{array}$ & $\begin{array}{l}\text { Field } \\
\text { Length }\end{array}$ & $\begin{array}{l}\text { Decimal } \\
\text { Places }\end{array}$ & $\begin{array}{l}\text { Required } \\
\text { Field }\end{array}$ & $\begin{array}{l}\text { Primary } \\
\text { Key }\end{array}$ & Definition \\
\hline CWHRHabitat & varchar & 50 & 0 & $\mathrm{~N}$ & $\mathrm{~N}$ & $\begin{array}{l}\text { California Wildlife Habitat Relationship (CWRH) System } \\
\text { habitat type. }\end{array}$ \\
\hline CWHRHabitatCode & varchar & 3 & 0 & $\mathrm{Y}$ & $\mathrm{Y}$ & California Wildlife Habitat Relationship System code. \\
\hline CWHRHabitatDesc & varchar & 250 & 0 & $\mathrm{~N}$ & $\mathrm{~N}$ & $\begin{array}{l}\text { Description of California Wildlife Habitat Relationship } \\
\text { System habitat type. }\end{array}$ \\
\hline
\end{tabular}

\section{Affiliate Table Look Ups}

luAffiliate Type

\begin{tabular}{lcccccl}
\hline \multicolumn{1}{c}{ Field Name } & $\begin{array}{c}\text { Data } \\
\text { Type }\end{array}$ & $\begin{array}{c}\text { Field } \\
\text { Length }\end{array}$ & $\begin{array}{c}\text { Decimal } \\
\text { Places }\end{array}$ & $\begin{array}{c}\text { Required } \\
\text { Field }\end{array}$ & $\begin{array}{c}\text { Primary } \\
\text { Key }\end{array}$ & Definition \\
\hline AffiliateType & varchar & 50 & 0 & $\mathrm{Y}$ & $\mathrm{N}$ & Type of organization affiliate belongs to. \\
AffiliateTypeID & int & 0 & 0 & $\mathrm{Y}$ & $\mathrm{Y}$ & AffiliateType record identifier. \\
Description & varchar & 255 & 0 & $\mathrm{~N}$ & $\mathrm{~N}$ & Description of the organization. \\
\hline
\end{tabular}


luCountry

\begin{tabular}{lcccccl}
\hline \multicolumn{1}{c}{ Field Name } & $\begin{array}{c}\text { Data } \\
\text { Type }\end{array}$ & $\begin{array}{c}\text { Field } \\
\text { Length }\end{array}$ & $\begin{array}{c}\text { Decimal } \\
\text { Places }\end{array}$ & $\begin{array}{c}\text { Required } \\
\text { Field }\end{array}$ & $\begin{array}{c}\text { Primary } \\
\text { Key }\end{array}$ & Definition \\
\hline Country & nvarchar & 255 & 0 & $\mathrm{Y}$ & $\mathrm{N}$ & Full name of country. \\
CountryCode & char & 2 & 0 & $\mathrm{Y}$ & $\mathrm{Y}$ & Two letter country code. \\
CountryCodeA3 & char & 3 & 0 & $\mathrm{~N}$ & $\mathrm{~N}$ & Three letter country code. \\
\hline
\end{tabular}

luState

\begin{tabular}{lcccccl}
\hline $\begin{array}{c}\text { Field } \\
\text { Name }\end{array}$ & $\begin{array}{c}\text { Data } \\
\text { Type }\end{array}$ & $\begin{array}{c}\text { Field } \\
\text { Length }\end{array}$ & $\begin{array}{c}\text { Decimal } \\
\text { Places }\end{array}$ & $\begin{array}{c}\text { Required } \\
\text { Field }\end{array}$ & $\begin{array}{c}\text { Primary } \\
\text { Key }\end{array}$ & Definition \\
\hline State & varchar & 50 & 0 & $\mathrm{Y}$ & $\mathrm{N}$ & State in which the site is located. \\
StateCode & char & 2 & 0 & $\mathrm{Y}$ & $\mathrm{Y}$ & Two letter abbreviation of the State. \\
\hline
\end{tabular}

\section{Aquatic Habitat Table Look Ups}

luExtentInundation

\begin{tabular}{lccccccl}
\hline \multicolumn{1}{c}{ Field Name } & $\begin{array}{c}\text { Data } \\
\text { Type }\end{array}$ & $\begin{array}{c}\text { Field } \\
\text { Length }\end{array}$ & $\begin{array}{c}\text { Decimal } \\
\text { Places }\end{array}$ & $\begin{array}{c}\text { Required } \\
\text { Field }\end{array}$ & $\begin{array}{c}\text { Primary } \\
\text { Key }\end{array}$ & Definition \\
\hline ExtentInundation & varchar & 30 & 0 & $\mathrm{Y}$ & $\mathrm{N}$ & Percent range of site covered in water. \\
ExtentInundationID & int & 0 & 0 & $\mathrm{Y}$ & $\mathrm{Y}$ & ExtentInundation record identifier. \\
\hline
\end{tabular}

luStreamOrder

\begin{tabular}{lcccccc}
\hline Field Name & $\begin{array}{c}\text { Data } \\
\text { Type }\end{array}$ & $\begin{array}{c}\text { Field } \\
\text { Length }\end{array}$ & $\begin{array}{c}\text { Decimal } \\
\text { Places }\end{array}$ & $\begin{array}{c}\text { Required } \\
\text { Field }\end{array}$ & $\begin{array}{c}\text { Primary } \\
\text { Key }\end{array}$ & Definition \\
\hline StreamOrder & varchar & 100 & 0 & Y & N & Stream size based on a hierarchy of tributaries. \\
StreamOrderID & int & 0 & 0 & Y & Y & StreamOrder record identifier (hierarchy based). \\
StreamOrderMethodID & int & 0 & 0 & Y & N & $\begin{array}{c}\text { StreamOrderMethod record identifier. See } \\
\text { luStreamOrderMethodID table below. }\end{array}$ \\
\hline
\end{tabular}

IuStreamOrderMethodID

\begin{tabular}{lccccccl}
\hline \multicolumn{1}{c}{ Field Name } & $\begin{array}{c}\text { Data } \\
\text { Type }\end{array}$ & $\begin{array}{c}\text { Field } \\
\text { Length }\end{array}$ & $\begin{array}{c}\text { Decimal } \\
\text { Places }\end{array}$ & $\begin{array}{c}\text { Required } \\
\text { Field }\end{array}$ & $\begin{array}{c}\text { Primary } \\
\text { Key }\end{array}$ & Definition \\
\hline StreamOrderMethod & varchar & 25 & 0 & Y & N & Method used to determine stream order/hierarchy. \\
StreamOrderMethodID & int & 0 & 0 & Y & Y & StreamOrderMethod record identifier. \\
\hline
\end{tabular}

luWaterFlow

\begin{tabular}{lcrcccll}
\hline Field Name & $\begin{array}{c}\text { Data } \\
\text { Type }\end{array}$ & $\begin{array}{c}\text { Field } \\
\text { Length }\end{array}$ & $\begin{array}{c}\text { Decimal } \\
\text { Places }\end{array}$ & $\begin{array}{c}\text { Required } \\
\text { Field }\end{array}$ & $\begin{array}{c}\text { Primary } \\
\text { Key }\end{array}$ & Definition \\
\hline Description & varchar & 100 & 0 & $\mathrm{~N}$ & $\mathrm{~N}$ & Description of water flow rate. \\
WaterFlow & varchar & 20 & 0 & $\mathrm{Y}$ & $\mathrm{N}$ & Flow rate of the water. \\
WaterFlowID & int & 0 & 0 & $\mathrm{Y}$ & $\mathrm{Y}$ & WaterFlow record identifier. & \\
\hline
\end{tabular}

luWaterSource

\begin{tabular}{|c|c|c|c|c|c|c|}
\hline Field Name & $\begin{array}{l}\text { Data } \\
\text { Type }\end{array}$ & $\begin{array}{c}\text { Field } \\
\text { Length }\end{array}$ & $\begin{array}{c}\text { Decimal } \\
\text { Places }\end{array}$ & $\begin{array}{l}\text { Required } \\
\text { Field }\end{array}$ & $\begin{array}{c}\text { Primary } \\
\text { Key }\end{array}$ & Definition \\
\hline WaterSource & varchar & 30 & 0 & $\mathrm{Y}$ & $\mathrm{N}$ & Origin of water body. \\
\hline
\end{tabular}


Basking Areas Table Look Ups

luBaskingAreas

\begin{tabular}{lcccccc}
\hline Field Name & $\begin{array}{c}\text { Data } \\
\text { Type }\end{array}$ & $\begin{array}{c}\text { Field } \\
\text { Length }\end{array}$ & $\begin{array}{c}\text { Decimal } \\
\text { Places }\end{array}$ & $\begin{array}{c}\text { Required } \\
\text { Field }\end{array}$ & $\begin{array}{c}\text { Primary } \\
\text { Key }\end{array}$ & Definition \\
\hline BaskingArea & varchar & 20 & 0 & Y & Y & $\begin{array}{c}\text { Area above the surface of the water where sunny space is available for } \\
\text { animals to rest and sun themselves (for example, rocks, sunny banks). }\end{array}$ \\
\hline
\end{tabular}

luBaskingAreasContext

\begin{tabular}{ccccccc}
\hline $\begin{array}{c}\text { Field } \\
\text { Name }\end{array}$ & $\begin{array}{c}\text { Data } \\
\text { Type }\end{array}$ & $\begin{array}{c}\text { Field } \\
\text { Length }\end{array}$ & $\begin{array}{c}\text { Decimal } \\
\text { Places }\end{array}$ & $\begin{array}{c}\text { Required } \\
\text { Field }\end{array}$ & $\begin{array}{c}\text { Primary } \\
\text { Key }\end{array}$ & Definition \\
\hline Context & varchar & 20 & 0 & Y & Y & Area within habitat where basking area is observed. \\
\hline
\end{tabular}

\section{Behaviors Table Look Ups}

luBehavior

\begin{tabular}{cccccccc}
\hline $\begin{array}{c}\text { Field } \\
\text { Name }\end{array}$ & $\begin{array}{c}\text { Data } \\
\text { Type }\end{array}$ & $\begin{array}{c}\text { Field } \\
\text { Length }\end{array}$ & $\begin{array}{c}\text { Decimal } \\
\text { Places }\end{array}$ & $\begin{array}{c}\text { Required } \\
\text { Field }\end{array}$ & $\begin{array}{c}\text { Primary } \\
\text { Key }\end{array}$ & Definition \\
\hline Behavior & varchar & 50 & 0 & $\mathrm{~N}$ & $\mathrm{Y}$ & Behavior of observed taxon. & \\
\hline
\end{tabular}

Collection Table Look Ups

luCollectionType

\begin{tabular}{ccccccc}
\hline Field Name & $\begin{array}{c}\text { Data } \\
\text { Type }\end{array}$ & $\begin{array}{c}\text { Field } \\
\text { Length }\end{array}$ & $\begin{array}{c}\text { Decimal } \\
\text { Places }\end{array}$ & $\begin{array}{c}\text { Required } \\
\text { Field }\end{array}$ & $\begin{array}{c}\text { Primary } \\
\text { Key }\end{array}$ & Definition \\
\hline CollectionType & varchar & 15 & 0 & Y & Y & Description of physical type of collection. \\
\hline
\end{tabular}

luCurationStatus

\begin{tabular}{ccccccc}
\hline Field Name & $\begin{array}{c}\text { Data } \\
\text { Type }\end{array}$ & $\begin{array}{c}\text { Field } \\
\text { Length }\end{array}$ & $\begin{array}{c}\text { Decimal } \\
\text { Places }\end{array}$ & $\begin{array}{c}\text { Required } \\
\text { Field }\end{array}$ & $\begin{array}{c}\text { Primary } \\
\text { Key }\end{array}$ & Definition \\
\hline CurationStatus & varchar & 15 & 0 & Y & Y & Current lab-workflow stage for this collection. \\
\hline
\end{tabular}

luPreservationMethod

\begin{tabular}{lcccccc}
\hline \multicolumn{1}{c}{ Field Name } & $\begin{array}{c}\text { Data } \\
\text { Type }\end{array}$ & $\begin{array}{c}\text { Field } \\
\text { Length }\end{array}$ & $\begin{array}{c}\text { Decimal } \\
\text { Places }\end{array}$ & $\begin{array}{c}\text { Required } \\
\text { Field }\end{array}$ & $\begin{array}{c}\text { Primary } \\
\text { Key }\end{array}$ & Definition \\
\hline Description & varchar & 20 & 0 & $\mathrm{Y}$ & $\mathrm{N}$ & Description of medium used to preserve the physical specimen. \\
PresMethodID & char & 1 & 0 & $\mathrm{Y}$ & $\mathrm{Y}$ & PreservationMethod record identifier. \\
\hline
\end{tabular}

TissType

\begin{tabular}{lccccccl}
\hline Field Name & $\begin{array}{c}\text { Data } \\
\text { Type }\end{array}$ & $\begin{array}{c}\text { Field } \\
\text { Length }\end{array}$ & $\begin{array}{c}\text { Decimal } \\
\text { Places }\end{array}$ & $\begin{array}{c}\text { Required } \\
\text { Field }\end{array}$ & $\begin{array}{c}\text { Primary } \\
\text { Key }\end{array}$ & Definition \\
\hline TissueDesc & varchar & 50 & 0 & $\mathrm{Y}$ & $\mathrm{N}$ & Description of tissue type. & \\
TissueTypeID & char & 2 & 0 & $\mathrm{Y}$ & $\mathrm{Y}$ & TissueType record identifier. & \\
\hline
\end{tabular}


Collection_Affiliate Table Look Ups

luCollectionUses

\begin{tabular}{cccccccc}
\hline Field Name & $\begin{array}{c}\text { Data } \\
\text { Type }\end{array}$ & $\begin{array}{c}\text { Field } \\
\text { Length }\end{array}$ & $\begin{array}{c}\text { Decimal } \\
\text { Places }\end{array}$ & $\begin{array}{c}\text { Required } \\
\text { Field }\end{array}$ & $\begin{array}{c}\text { Primary } \\
\text { Key }\end{array}$ & Definition \\
\hline CollectionUse & varchar & 25 & 0 & Y & Y & Use of the collected sample for the affiliate who requested it. \\
\hline luReturnExpected & Data & Field & Decimal \\
Type & Length & Places & $\begin{array}{c}\text { Required } \\
\text { Field }\end{array}$ & $\begin{array}{c}\text { Primary } \\
\text { Key }\end{array}$ & Definition \\
\hline Field Name & varchar & 10 & 0 & Y & Y & $\begin{array}{c}\text { Is the item expected to be returned to the original lab, or status of } \\
\text { returned item. }\end{array}$ \\
\hline ReturnExpected & & & & & & &
\end{tabular}

\section{DistanceTo Table Look Ups}

luDistanceToType

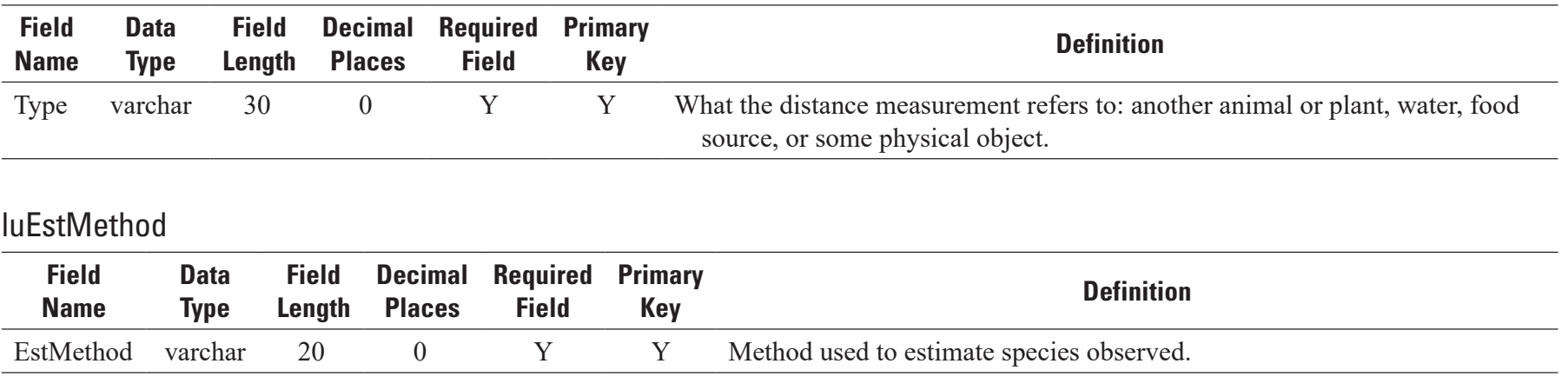

\section{EstimateSeries Table Look Ups}

luGender

\begin{tabular}{lcccccll}
\hline \multirow{2}{*}{ Field Name } & $\begin{array}{c}\text { Data } \\
\text { Type }\end{array}$ & $\begin{array}{c}\text { Field } \\
\text { Length }\end{array}$ & $\begin{array}{c}\text { Decimal } \\
\text { Places }\end{array}$ & $\begin{array}{c}\text { Required } \\
\text { Field }\end{array}$ & $\begin{array}{c}\text { Primary } \\
\text { Key }\end{array}$ & Definition \\
\hline Description & varchar & 255 & 0 & $\mathrm{~N}$ & $\mathrm{~N}$ & Description of gender. & \\
Gender & varchar & 30 & 0 & $\mathrm{Y}$ & $\mathrm{N}$ & Gender of taxon. \\
GenderID & int & 0 & 0 & $\mathrm{Y}$ & $\mathrm{Y}$ & Gender record identifier. & \\
\hline
\end{tabular}

luLifeStage

\begin{tabular}{lcccccll}
\hline \multirow{2}{*}{ Field Name } & $\begin{array}{c}\text { Data } \\
\text { Type }\end{array}$ & $\begin{array}{c}\text { Field } \\
\text { Length }\end{array}$ & $\begin{array}{c}\text { Decimal } \\
\text { Places }\end{array}$ & $\begin{array}{c}\text { Required } \\
\text { Field }\end{array}$ & $\begin{array}{c}\text { Primary } \\
\text { Key }\end{array}$ & Definition \\
\hline Description & varchar & 350 & 0 & $\mathrm{~N}$ & $\mathrm{~N}$ & Description of life stage. & \\
LifeStage & varchar & 20 & 0 & $\mathrm{Y}$ & $\mathrm{N}$ & Life Stage of taxon. \\
LifeStageID & int & 0 & 0 & $\mathrm{Y}$ & $\mathrm{Y}$ & LifeStage record identifier. & \\
\hline
\end{tabular}

luParameterType

\begin{tabular}{ccccccc}
\hline $\begin{array}{c}\text { Field } \\
\text { Name }\end{array}$ & $\begin{array}{c}\text { Data } \\
\text { Type }\end{array}$ & $\begin{array}{c}\text { Field } \\
\text { Length }\end{array}$ & $\begin{array}{c}\text { Decimal } \\
\text { Places }\end{array}$ & $\begin{array}{c}\text { Required } \\
\text { Field }\end{array}$ & $\begin{array}{c}\text { Primary } \\
\text { Key }\end{array}$ & Definition \\
\hline Parameter & varchar & 50 & 0 & Y & Y & Parameter to which the estimate applies. \\
\hline
\end{tabular}




\section{FocalSpeciesList Table Look Ups}

luFocalSpeciesPurpose

\begin{tabular}{cccccccc}
\hline Field Name & $\begin{array}{c}\text { Data } \\
\text { Type }\end{array}$ & $\begin{array}{c}\text { Field } \\
\text { Length }\end{array}$ & $\begin{array}{c}\text { Decimal } \\
\text { Places }\end{array}$ & $\begin{array}{c}\text { Required } \\
\text { Field }\end{array}$ & $\begin{array}{c}\text { Primary } \\
\text { Key }\end{array}$ & Definition \\
\hline FocalSpeciesPurpose & varchar & 40 & 0 & $\mathrm{Y}$ & $\mathrm{Y}$ & Purpose of the focal species studied in study. \\
\hline luPopulationDesignation & & & & & & & \\
\hline Field Name & Data & Field & Decimal \\
Type & Length & Places & $\begin{array}{c}\text { Required } \\
\text { Field }\end{array}$ & $\begin{array}{c}\text { Primary } \\
\text { Key }\end{array}$ & Definition \\
\hline PopulationDesignation & varchar & 10 & 0 & $\mathrm{Y}$ & $\mathrm{Y}$ & $\begin{array}{c}\text { Multiple Species Conservation Plan (MSCP) Population } \\
\text { designation. }\end{array}$ \\
\hline
\end{tabular}

\section{Funding/FundingProgram Table Look Ups}

luFundingType

\begin{tabular}{lcccccl}
\hline \multicolumn{1}{c}{ Field Name } & $\begin{array}{c}\text { Data } \\
\text { Type }\end{array}$ & $\begin{array}{c}\text { Field } \\
\text { Length }\end{array}$ & $\begin{array}{c}\text { Decimal } \\
\text { Places }\end{array}$ & $\begin{array}{c}\text { Required } \\
\text { Field }\end{array}$ & $\begin{array}{c}\text { Primary } \\
\text { Key }\end{array}$ & Definition \\
\hline FundingType & varchar & 50 & 0 & $\mathrm{~N}$ & $\mathrm{~N}$ & Type of funding for project. \\
FundingTypeID & int & 0 & 0 & $\mathrm{Y}$ & $\mathrm{Y}$ & FundingType record identifier. \\
\hline
\end{tabular}

\section{GrantMgtActions Table Look Ups}

luFundingAction

\begin{tabular}{lcccccl}
\hline \multicolumn{1}{c}{ Field Name } & $\begin{array}{c}\text { Data } \\
\text { Type }\end{array}$ & $\begin{array}{c}\text { Field } \\
\text { Length }\end{array}$ & $\begin{array}{c}\text { Decimal } \\
\text { Places }\end{array}$ & $\begin{array}{c}\text { Required } \\
\text { Field }\end{array}$ & $\begin{array}{c}\text { Primary } \\
\text { Key }\end{array}$ & Definition \\
\hline Description & varchar & 255 & 0 & $\mathrm{~N}$ & $\mathrm{~N}$ & Description of the funding action. \\
FundingAction & varchar & 25 & 0 & $\mathrm{Y}$ & $\mathrm{N}$ & Administrative action associated with management action. \\
FundingActionID & int & 0 & 0 & $\mathrm{Y}$ & $\mathrm{Y}$ & FundingAction record identifier. \\
\hline
\end{tabular}

\section{HabitatObservation Table Look Ups}

luCHWRHabitat

\begin{tabular}{|c|c|c|c|c|c|c|}
\hline Field Name & $\begin{array}{l}\text { Data } \\
\text { Type }\end{array}$ & $\begin{array}{l}\text { Field } \\
\text { Length }\end{array}$ & $\begin{array}{l}\text { Decimal } \\
\text { Places }\end{array}$ & $\begin{array}{l}\text { Required } \\
\text { Field }\end{array}$ & $\begin{array}{l}\text { Primary } \\
\text { Key }\end{array}$ & Definition \\
\hline CWHRHabitat & varchar & 50 & 0 & $\mathrm{~N}$ & $\mathrm{~N}$ & $\begin{array}{l}\text { California Wildlife Habitat Relationship (CWHR) System } \\
\text { habitat type. }\end{array}$ \\
\hline CWHRHabitatCode & varchar & 3 & 0 & $\mathrm{Y}$ & $\mathrm{Y}$ & California Wildlife Habitat Relationship System code. \\
\hline CWHRHabitatDesc & varchar & 250 & 0 & $\mathrm{~N}$ & $\mathrm{~N}$ & $\begin{array}{l}\text { Description of California Wildlife Habitat Relationship } \\
\text { System habitat type. }\end{array}$ \\
\hline
\end{tabular}

luRoadSurface

\begin{tabular}{lcrcccl}
\hline \multicolumn{1}{c}{ Field Name } & $\begin{array}{c}\text { Data } \\
\text { Type }\end{array}$ & $\begin{array}{c}\text { Field } \\
\text { Length }\end{array}$ & $\begin{array}{c}\text { Decimal } \\
\text { Places }\end{array}$ & $\begin{array}{c}\text { Required } \\
\text { Field }\end{array}$ & $\begin{array}{c}\text { Primary } \\
\text { Key }\end{array}$ & Definition \\
\hline Description & varchar & 255 & 0 & $\mathrm{~N}$ & $\mathrm{~N}$ & Description of RoadSurface condition. \\
RoadSurface & varchar & 50 & 0 & $\mathrm{Y}$ & $\mathrm{N}$ & Level of moisture on road surface in observed habitat. \\
RoadSurfaceCode & varchar & 2 & 0 & $\mathrm{Y}$ & $\mathrm{Y}$ & RoadSurface identifier code. \\
\hline
\end{tabular}


Permanency

\begin{tabular}{lccccccl}
\hline \multirow{2}{*}{ Field Name } & $\begin{array}{c}\text { Data } \\
\text { Type }\end{array}$ & $\begin{array}{c}\text { Field } \\
\text { Length }\end{array}$ & $\begin{array}{c}\text { Decimal } \\
\text { Places }\end{array}$ & $\begin{array}{c}\text { Required } \\
\text { Field }\end{array}$ & $\begin{array}{c}\text { Primary } \\
\text { Key }\end{array}$ & Definition \\
\hline Permanency & varchar & 40 & 0 & $\mathrm{Y}$ & $\mathrm{N}$ & Permanency of water body. & \\
PID & char & 1 & 0 & $\mathrm{Y}$ & $\mathrm{Y}$ & Permanency record identifier. & \\
\hline
\end{tabular}

\section{HabitatObservation_Feature Table Look Ups}

luHabitatFeatureType

\begin{tabular}{lcccccl}
\hline \multicolumn{1}{c}{ Field Name } & $\begin{array}{c}\text { Data } \\
\text { Type }\end{array}$ & $\begin{array}{c}\text { Field } \\
\text { Length }\end{array}$ & $\begin{array}{c}\text { Decimal } \\
\text { Places }\end{array}$ & $\begin{array}{c}\text { Required } \\
\text { Field }\end{array}$ & $\begin{array}{c}\text { Primary } \\
\text { Key }\end{array}$ & Definition \\
\hline Description & varchar & 255 & 0 & $\mathrm{~N}$ & $\mathrm{~N}$ & Description of habitat feature type. \\
HabitatFeatureType & varchar & 30 & 0 & $\mathrm{Y}$ & $\mathrm{N}$ & Type of feature observed within habitat. \\
HabitatFeatureTypeID & int & 0 & 0 & $\mathrm{Y}$ & $\mathrm{Y}$ & HabitatFeatureType record identifier. \\
\hline
\end{tabular}

\section{HabitatObservation_GroundCover Table Look Ups}

luGroundCover

\begin{tabular}{lcccccl}
\hline \multicolumn{1}{c}{ Field Name } & $\begin{array}{c}\text { Data } \\
\text { Type }\end{array}$ & $\begin{array}{c}\text { Field } \\
\text { Length }\end{array}$ & $\begin{array}{c}\text { Decimal } \\
\text { Places }\end{array}$ & $\begin{array}{c}\text { Required } \\
\text { Field }\end{array}$ & $\begin{array}{c}\text { Primary } \\
\text { Key }\end{array}$ & Definition \\
\hline GroundCover & varchar & 50 & 0 & $\mathrm{Y}$ & $\mathrm{N}$ & Type of ground cover observed in habitat. \\
GroundCoverCode & varchar & 5 & 0 & $\mathrm{~N}$ & $\mathrm{~N}$ & GroundCover abbreviation/code. \\
GroundCoverID & int & 0 & 0 & $\mathrm{Y}$ & $\mathrm{Y}$ & GroundCover record identifier. \\
\hline
\end{tabular}

\section{HabitatObservation_LandUse Table Look Ups}

luLandUse

\begin{tabular}{lcccccl}
\hline Field Name & $\begin{array}{c}\text { Data } \\
\text { Type }\end{array}$ & $\begin{array}{c}\text { Field } \\
\text { Length }\end{array}$ & $\begin{array}{c}\text { Decimal } \\
\text { Places }\end{array}$ & $\begin{array}{c}\text { Required } \\
\text { Field }\end{array}$ & $\begin{array}{c}\text { Primary } \\
\text { Key }\end{array}$ & Definition \\
\hline Landuse & varchar & 50 & 0 & $\mathrm{Y}$ & $\mathrm{N}$ & The primary function of the land. \\
LanduseCode & varchar & 3 & 0 & $\mathrm{~N}$ & $\mathrm{~N}$ & Abbreviation/code of land use. \\
LanduseID & int & 0 & 0 & $\mathrm{Y}$ & $\mathrm{Y}$ & Landuse record identifier. \\
\hline
\end{tabular}

\section{HabitatObservation_SedChemical Table Look Ups}

IuSedimentationChemical

\begin{tabular}{lcccccl}
\hline \multicolumn{1}{c}{ Field Name } & $\begin{array}{c}\text { Data } \\
\text { Type }\end{array}$ & $\begin{array}{c}\text { Field } \\
\text { Length }\end{array}$ & $\begin{array}{c}\text { Decimal } \\
\text { Places }\end{array}$ & $\begin{array}{c}\text { Required } \\
\text { Field }\end{array}$ & $\begin{array}{c}\text { Primary } \\
\text { Key }\end{array}$ & Definition \\
\hline Description & varchar & 100 & 0 & $\mathrm{~N}$ & $\mathrm{~N}$ & Description of the chemical found in the sediment. \\
SedimentationChemical & varchar & 50 & 0 & $\mathrm{Y}$ & $\mathrm{N}$ & Chemical found in the sediment within the habitat. \\
SedimentationChemicalID & int & 0 & 0 & $\mathrm{Y}$ & $\mathrm{Y}$ & SedimentationChemical record identifier. \\
\hline
\end{tabular}




\section{HabitatObservation_Substrate Table Look Ups}

luSubstrate

\begin{tabular}{lcccccl}
\hline \multicolumn{1}{c}{ Field Name } & $\begin{array}{c}\text { Data } \\
\text { Type }\end{array}$ & $\begin{array}{c}\text { Field } \\
\text { Length }\end{array}$ & $\begin{array}{c}\text { Decimal } \\
\text { Places }\end{array}$ & $\begin{array}{c}\text { Required } \\
\text { Field }\end{array}$ & $\begin{array}{c}\text { Primary } \\
\text { Key }\end{array}$ & Definition \\
\hline Description & varchar & 255 & 0 & $\mathrm{~N}$ & $\mathrm{~N}$ & Description of the observed substrate. \\
Substrate & varchar & 100 & 0 & $\mathrm{Y}$ & $\mathrm{N}$ & Type of substrate (surface composition) observed within habitat. \\
SubstrateCode & varchar & 2 & 0 & $\mathrm{~N}$ & $\mathrm{~N}$ & Code/abbreviation of observed substrate. \\
SubstrateID & int & 0 & 0 & $\mathrm{Y}$ & $\mathrm{Y}$ & Substrate record identifier. \\
\hline
\end{tabular}

luSubstrateContext

\begin{tabular}{lcccccl}
\hline \multirow{2}{*}{ Field Name } & $\begin{array}{c}\text { Data } \\
\text { Type }\end{array}$ & $\begin{array}{c}\text { Field } \\
\text { Length }\end{array}$ & $\begin{array}{c}\text { Decimal } \\
\text { Places }\end{array}$ & $\begin{array}{c}\text { Required } \\
\text { Field }\end{array}$ & $\begin{array}{c}\text { Primary } \\
\text { Key }\end{array}$ & Definition \\
\hline Description & varchar & 255 & 0 & $\mathrm{~N}$ & $\mathrm{~N}$ & Description of the Substrate Context. \\
SubstrateContext & varchar & 30 & 0 & $\mathrm{Y}$ & $\mathrm{N}$ & Area within habitat that substrate was found. \\
SubstrateContextCode & varchar & 2 & 0 & $\mathrm{Y}$ & $\mathrm{Y}$ & SubstrateContext abbreviation/code. \\
\hline
\end{tabular}

\section{HabitatObservation_VegetationTable Look Ups}

luHabitatObsVegContext

\begin{tabular}{ccccccc}
\hline $\begin{array}{c}\text { Field } \\
\text { Name }\end{array}$ & $\begin{array}{c}\text { Data } \\
\text { Type }\end{array}$ & $\begin{array}{c}\text { Field } \\
\text { Length }\end{array}$ & $\begin{array}{c}\text { Decimal } \\
\text { Places }\end{array}$ & $\begin{array}{c}\text { Required } \\
\text { Field }\end{array}$ & $\begin{array}{c}\text { Primary } \\
\text { Key }\end{array}$ & Definition \\
\hline Context & varchar & 20 & 0 & Y & Y & General area of vegetation observation in habitat. \\
\hline
\end{tabular}

luHabitatVegetationType

\begin{tabular}{|c|c|c|c|c|c|c|}
\hline Field Name & $\begin{array}{l}\text { Data } \\
\text { Type }\end{array}$ & $\begin{array}{l}\text { Field } \\
\text { Length }\end{array}$ & $\begin{array}{l}\text { Decimal } \\
\text { Places }\end{array}$ & $\begin{array}{l}\text { Required } \\
\text { Field }\end{array}$ & $\begin{array}{l}\text { Primary } \\
\text { Key }\end{array}$ & Definition \\
\hline HabitatVegetationType & varchar & 100 & 0 & $\mathrm{Y}$ & $\mathrm{N}$ & Type of vegetation found within habitat. \\
\hline $\begin{array}{l}\text { HabitatVegetationType } \\
\text { ID }\end{array}$ & int & 0 & 0 & Y & $\mathrm{Y}$ & HabitatVegetationType record identifier. \\
\hline
\end{tabular}

IndivAnimals Table Look Ups

luAgePelage

\begin{tabular}{lccccccl}
\hline \multicolumn{1}{c}{ Field Name } & $\begin{array}{c}\text { Data } \\
\text { Type }\end{array}$ & $\begin{array}{c}\text { Field } \\
\text { Length }\end{array}$ & $\begin{array}{c}\text { Decimal } \\
\text { Places }\end{array}$ & $\begin{array}{c}\text { Required } \\
\text { Field }\end{array}$ & $\begin{array}{c}\text { Primary } \\
\text { Key }\end{array}$ & Definition \\
\hline AgePelageCode & char & 2 & 0 & $\mathrm{Y}$ & $\mathrm{Y}$ & Abbreviation of age of animal. \\
Description & varchar & 25 & 0 & $\mathrm{Y}$ & $\mathrm{N}$ & Age of animal. & \\
\hline
\end{tabular}

luDisposition

\begin{tabular}{lcccccc}
\hline Field Name & $\begin{array}{c}\text { Data } \\
\text { Type }\end{array}$ & $\begin{array}{c}\text { Field } \\
\text { Length }\end{array}$ & $\begin{array}{c}\text { Decimal } \\
\text { Places }\end{array}$ & $\begin{array}{c}\text { Required } \\
\text { Field }\end{array}$ & $\begin{array}{c}\text { Primary } \\
\text { Key }\end{array}$ & Definition \\
\hline Disposition & varchar & 10 & 0 & Y & Y & Disposition (e.g. alive, dead) of animal. \\
\hline
\end{tabular}


luFate

\begin{tabular}{lcccccc}
\hline $\begin{array}{c}\text { Field } \\
\text { Name }\end{array}$ & $\begin{array}{c}\text { Data } \\
\text { Type }\end{array}$ & $\begin{array}{c}\text { Field } \\
\text { Length }\end{array}$ & $\begin{array}{c}\text { Decimal } \\
\text { Places }\end{array}$ & $\begin{array}{c}\text { Required } \\
\text { Field }\end{array}$ & $\begin{array}{c}\text { Primary } \\
\text { Key }\end{array}$ & Definition \\
\hline Fate & varchar & 15 & 0 & Y & Y & What happened to individual after capture? \\
\hline
\end{tabular}

luPubicSymphysis

\begin{tabular}{lcccccl}
\hline Field Name & $\begin{array}{c}\text { Data } \\
\text { Type }\end{array}$ & $\begin{array}{c}\text { Field } \\
\text { Length }\end{array}$ & $\begin{array}{c}\text { Decimal } \\
\text { Places }\end{array}$ & $\begin{array}{c}\text { Required } \\
\text { Field }\end{array}$ & $\begin{array}{c}\text { Primary } \\
\text { Key }\end{array}$ & Definition \\
\hline Description & varchar & 15 & 0 & $\mathrm{~N}$ & $\mathrm{~N}$ & Status of the pubic symphysis joint. \\
PubicSymID & char & 3 & 0 & $\mathrm{Y}$ & $\mathrm{Y}$ & PubicSymphysis record identifier. \\
\hline
\end{tabular}

luShwear

\begin{tabular}{lcccccl}
\hline \multirow{2}{*}{ Field Name } & $\begin{array}{c}\text { Data } \\
\text { Type }\end{array}$ & $\begin{array}{c}\text { Field } \\
\text { Length }\end{array}$ & $\begin{array}{c}\text { Decimal } \\
\text { Places }\end{array}$ & $\begin{array}{c}\text { Required } \\
\text { Field }\end{array}$ & $\begin{array}{c}\text { Primary } \\
\text { Key }\end{array}$ & Definition \\
\hline Description & varchar & 250 & 0 & $\mathrm{~N}$ & $\mathrm{~N}$ & Description of the shell wear age class. \\
ShwearID & numeric & 1 & 0 & $\mathrm{Y}$ & $\mathrm{Y}$ & Shell Wear age class. \\
\hline
\end{tabular}

luTeats

\begin{tabular}{lcccccl}
\hline Field Name & $\begin{array}{c}\text { Data } \\
\text { Type }\end{array}$ & $\begin{array}{c}\text { Field } \\
\text { Length }\end{array}$ & $\begin{array}{c}\text { Decimal } \\
\text { Places }\end{array}$ & $\begin{array}{c}\text { Required } \\
\text { Field }\end{array}$ & $\begin{array}{c}\text { Primary } \\
\text { Key }\end{array}$ & Definition \\
\hline Description & varchar & 15 & 0 & $\mathrm{Y}$ & $\mathrm{N}$ & Condition of teats. \\
TeatsID & char & 2 & 0 & $\mathrm{Y}$ & $\mathrm{Y}$ & Abbreviation of condition of teats. \\
\hline
\end{tabular}

\section{IndividualAnimalldentifier Table Look Ups}

IDType

\begin{tabular}{lccccccl}
\hline Field Name & $\begin{array}{c}\text { Data } \\
\text { Type }\end{array}$ & $\begin{array}{c}\text { Field } \\
\text { Length }\end{array}$ & $\begin{array}{c}\text { Decimal } \\
\text { Places }\end{array}$ & $\begin{array}{c}\text { Required } \\
\text { Field }\end{array}$ & $\begin{array}{c}\text { Primary } \\
\text { Key }\end{array}$ & Definition \\
\hline Description & varchar & 30 & 0 & $\mathrm{Y}$ & $\mathrm{N}$ & Type of tag or identification marker used on animal. \\
IDType & varchar & 2 & 0 & $\mathrm{Y}$ & $\mathrm{Y}$ & IDType record identifier. & \\
\hline
\end{tabular}

\section{Keywords Table Look Ups}

luKeywordType

\begin{tabular}{lcccccc}
\hline $\begin{array}{c}\text { Field } \\
\text { Name }\end{array}$ & $\begin{array}{c}\text { Data } \\
\text { Type }\end{array}$ & $\begin{array}{c}\text { Field } \\
\text { Length }\end{array}$ & $\begin{array}{c}\text { Decimal } \\
\text { Places }\end{array}$ & $\begin{array}{c}\text { Required } \\
\text { Field }\end{array}$ & $\begin{array}{c}\text { Primary } \\
\text { Key }\end{array}$ & Definition \\
\hline Type & varchar & 25 & 0 & $\mathrm{~N}$ & $\mathrm{~N}$ & Optional way to classify and/or organize the keywords into lists, etc. \\
\hline
\end{tabular}

\section{Maintenance Table Look Ups}

luFacilityType

\begin{tabular}{lcccccl}
\hline \multicolumn{1}{c}{ Field Name } & $\begin{array}{c}\text { Data } \\
\text { Type }\end{array}$ & $\begin{array}{c}\text { Field } \\
\text { Length }\end{array}$ & $\begin{array}{c}\text { Decimal } \\
\text { Places }\end{array}$ & $\begin{array}{c}\text { Required } \\
\text { Field }\end{array}$ & $\begin{array}{c}\text { Primary } \\
\text { Key }\end{array}$ & Definition \\
\hline Description & varchar & 255 & 0 & $\mathrm{~N}$ & $\mathrm{~N}$ & Description of the facility. \\
FacilityType & varchar & 25 & 0 & $\mathrm{Y}$ & $\mathrm{N}$ & Type of facility. \\
FacilityTypeID & int & 0 & 0 & $\mathrm{Y}$ & $\mathrm{Y}$ & FacilityType record identifier. \\
\hline
\end{tabular}


luMaintType

\begin{tabular}{lcccccl}
\hline \multirow{2}{*}{ Field Name } & $\begin{array}{c}\text { Data } \\
\text { Type }\end{array}$ & $\begin{array}{c}\text { Field } \\
\text { Length }\end{array}$ & $\begin{array}{c}\text { Decimal } \\
\text { Places }\end{array}$ & $\begin{array}{c}\text { Required } \\
\text { Field }\end{array}$ & $\begin{array}{c}\text { Primary } \\
\text { Key }\end{array}$ & Definition \\
\hline Description & varchar & 255 & 0 & $\mathrm{~N}$ & $\mathrm{~N}$ & Description of the maintenance type. \\
MaintType & varchar & 25 & 0 & $\mathrm{Y}$ & $\mathrm{N}$ & Type of maintenance conducted. \\
MaintTypeID & int & 0 & 0 & $\mathrm{Y}$ & $\mathrm{Y}$ & MaintType record identifier. \\
\hline
\end{tabular}

luMaterialType

\begin{tabular}{lcrccccl}
\hline \multicolumn{1}{c}{ Field Name } & $\begin{array}{c}\text { Data } \\
\text { Type }\end{array}$ & $\begin{array}{c}\text { Field } \\
\text { Length }\end{array}$ & $\begin{array}{c}\text { Decimal } \\
\text { Places }\end{array}$ & $\begin{array}{c}\text { Required } \\
\text { Field }\end{array}$ & $\begin{array}{c}\text { Primary } \\
\text { Key }\end{array}$ & Definition \\
\hline Description & varchar & 255 & 0 & $\mathrm{~N}$ & $\mathrm{~N}$ & Description of the material type. \\
MaterialType & varchar & 25 & 0 & $\mathrm{Y}$ & $\mathrm{N}$ & Type of material used for maintenance. \\
MaterialTypeID & int & 0 & 0 & $\mathrm{Y}$ & $\mathrm{Y}$ & MaterialType record identifier. \\
Notes & varchar & 255 & 0 & $\mathrm{~N}$ & $\mathrm{~N}$ & Notes about the material type. \\
Supplier & int & 0 & 0 & $\mathrm{~N}$ & $\mathrm{~N}$ & $\begin{array}{c}\text { Associated Affiliate record identifier of person/organization who } \\
\text { supplied material. }\end{array}$ \\
& & & & & &
\end{tabular}

\section{MaintenancePoint Table Look Ups}

luMaintPointType

\begin{tabular}{lcccccc}
\hline \multicolumn{1}{c}{ Field Name } & $\begin{array}{c}\text { Data } \\
\text { Type }\end{array}$ & $\begin{array}{c}\text { Field } \\
\text { Length }\end{array}$ & $\begin{array}{c}\text { Decimal } \\
\text { Places }\end{array}$ & $\begin{array}{c}\text { Required } \\
\text { Field }\end{array}$ & $\begin{array}{c}\text { Primary } \\
\text { Key }\end{array}$ & Definition \\
\hline Description & varchar & 50 & 0 & $\mathrm{~N}$ & $\mathrm{~N}$ & Description of the maintenance point type. \\
MaintPointType & varchar & 15 & 0 & $\mathrm{Y}$ & $\mathrm{Y}$ & $\begin{array}{c}\text { To what the maintenance point/spatial feature refers (fence beginning } \\
\text { point, fence end point, and so forth). }\end{array}$ \\
\hline
\end{tabular}

\section{ManagementActivities Table Look Ups}

luActivityType

\begin{tabular}{lcccccl}
\hline \multicolumn{1}{c}{ Field Name } & $\begin{array}{c}\text { Data } \\
\text { Type }\end{array}$ & $\begin{array}{c}\text { Field } \\
\text { Length }\end{array}$ & $\begin{array}{c}\text { Decimal } \\
\text { Places }\end{array}$ & $\begin{array}{c}\text { Required } \\
\text { Field }\end{array}$ & $\begin{array}{c}\text { Primary } \\
\text { Key }\end{array}$ & Definition \\
\hline ActivityType & varchar & 50 & 0 & $\mathrm{Y}$ & $\mathrm{N}$ & Type of management activity. \\
ActivityTypeID & int & 0 & 0 & $\mathrm{Y}$ & $\mathrm{Y}$ & ActivityType record identifier. \\
Description & varchar & 255 & 0 & $\mathrm{~N}$ & $\mathrm{~N}$ & Description of the management activity type. \\
\hline
\end{tabular}

\section{MgmtActivityMonitoring Table Look Ups}

luMonitoringType

\begin{tabular}{lcccccl}
\hline \multicolumn{1}{c}{ Field Name } & $\begin{array}{c}\text { Data } \\
\text { Type }\end{array}$ & $\begin{array}{c}\text { Field } \\
\text { Length }\end{array}$ & $\begin{array}{c}\text { Decimal } \\
\text { Places }\end{array}$ & $\begin{array}{c}\text { Required } \\
\text { Field }\end{array}$ & $\begin{array}{c}\text { Primary } \\
\text { Key }\end{array}$ & Definition \\
\hline Description & varchar & 255 & 0 & $\mathrm{~N}$ & $\mathrm{~N}$ & Description of the type of monitoring. \\
MonitoringType & varchar & 25 & 0 & $\mathrm{Y}$ & $\mathrm{N}$ & Proposed monitoring type. \\
MonitoringTypeID & int & 0 & 0 & $\mathrm{Y}$ & $\mathrm{Y}$ & MonitoringType record identifier. \\
\hline
\end{tabular}


MgtPlanDetails Table Look Ups

luMgtType

\begin{tabular}{lcrcccl}
\hline \multicolumn{1}{c}{ Field Name } & $\begin{array}{c}\text { Data } \\
\text { Type }\end{array}$ & $\begin{array}{c}\text { Field } \\
\text { Length }\end{array}$ & $\begin{array}{c}\text { Decimal } \\
\text { Places }\end{array}$ & $\begin{array}{c}\text { Required } \\
\text { Field }\end{array}$ & $\begin{array}{c}\text { Primary } \\
\text { Key }\end{array}$ & \multicolumn{2}{c}{ Definition } \\
\hline Description & varchar & 255 & 0 & $\mathrm{~N}$ & $\mathrm{~N}$ & Description of the management type. \\
ManagementType & varchar & 50 & 0 & $\mathrm{Y}$ & $\mathrm{N}$ & Type of management. \\
MgtTypeID & int & 0 & 0 & $\mathrm{Y}$ & $\mathrm{Y}$ & MgtType record identifier. \\
\hline
\end{tabular}

\section{MgtProjectStatus Table Look Ups}

luMgtProjectStatus

\begin{tabular}{lccccccl}
\hline \multirow{2}{*}{ Field Name } & $\begin{array}{c}\text { Data } \\
\text { Type }\end{array}$ & $\begin{array}{c}\text { Field } \\
\text { Length }\end{array}$ & $\begin{array}{c}\text { Decimal } \\
\text { Places }\end{array}$ & $\begin{array}{c}\text { Required } \\
\text { Field }\end{array}$ & $\begin{array}{c}\text { Primary } \\
\text { Key }\end{array}$ & Definition \\
\hline Description & varchar & 255 & 0 & $\mathrm{Y}$ & $\mathrm{N}$ & Description of the management project status. \\
Status & varchar & 15 & 0 & $\mathrm{Y}$ & $\mathrm{Y}$ & Status of the management project. \\
\hline
\end{tabular}

luMilestoneType

\begin{tabular}{lcccccl}
\hline \multicolumn{1}{c}{ Field Name } & $\begin{array}{c}\text { Data } \\
\text { Type }\end{array}$ & $\begin{array}{c}\text { Field } \\
\text { Length }\end{array}$ & $\begin{array}{c}\text { Decimal } \\
\text { Places }\end{array}$ & $\begin{array}{c}\text { Required } \\
\text { Field }\end{array}$ & $\begin{array}{c}\text { Primary } \\
\text { Key }\end{array}$ & Definition \\
\hline Description & varchar & 50 & 0 & $\mathrm{~N}$ & $\mathrm{~N}$ & Type of milestone used to gauge the success of the project. \\
MilestoneTypeID & smallint & 5 & 0 & $\mathrm{Y}$ & $\mathrm{N}$ & Milestone Type record identifier. \\
\hline
\end{tabular}

\section{NestEntranceDetails Table Look Ups}

luDirection

\begin{tabular}{lccccccl}
\hline Field Name & $\begin{array}{c}\text { Data } \\
\text { Type }\end{array}$ & $\begin{array}{c}\text { Field } \\
\text { Length }\end{array}$ & $\begin{array}{c}\text { Decimal } \\
\text { Places }\end{array}$ & $\begin{array}{c}\text { Required } \\
\text { Field }\end{array}$ & $\begin{array}{c}\text { Primary } \\
\text { Key }\end{array}$ & Definition \\
\hline Direction & varchar & 20 & 0 & $\mathrm{Y}$ & $\mathrm{N}$ & Cardinal direction. & \\
DirectionCode & varchar & 3 & 0 & $\mathrm{Y}$ & $\mathrm{Y}$ & Abbreviation for cardinal direction. \\
\hline
\end{tabular}

\section{ObservationDetectionMethods Table Look Ups}

luDetectionMethod

\begin{tabular}{lcccccl}
\hline \multicolumn{1}{c}{ Field Name } & $\begin{array}{c}\text { Data } \\
\text { Type }\end{array}$ & $\begin{array}{c}\text { Field } \\
\text { Length }\end{array}$ & $\begin{array}{c}\text { Decimal } \\
\text { Places }\end{array}$ & $\begin{array}{c}\text { Required } \\
\text { Field }\end{array}$ & $\begin{array}{c}\text { Primary } \\
\text { Key }\end{array}$ & Definition \\
\hline DetectionMethod & varchar & 50 & 0 & $\mathrm{Y}$ & $\mathrm{N}$ & How the observed taxon was detected. \\
DetectionMethodCode & char & 3 & 0 & $\mathrm{Y}$ & $\mathrm{N}$ & Code for Detection Method. \\
DetectionMethodID & int & 0 & 0 & $\mathrm{Y}$ & $\mathrm{Y}$ & DetectionMethod record identifier. \\
\hline
\end{tabular}

\section{ObservationVerification Table Look Ups}

luVerifMethods

\begin{tabular}{lcccccc}
\hline Field Name & $\begin{array}{c}\text { Data } \\
\text { Type }\end{array}$ & $\begin{array}{c}\text { Field } \\
\text { Length }\end{array}$ & $\begin{array}{c}\text { Decimal } \\
\text { Places }\end{array}$ & $\begin{array}{c}\text { Required } \\
\text { Field }\end{array}$ & $\begin{array}{c}\text { Primary } \\
\text { Key }\end{array}$ & Definition \\
\hline VerifMethod & varchar & 50 & 0 & Y & Y & Method used to verify the species identification. \\
\hline
\end{tabular}


Photolnfo Table Look Ups

luPhotoQuality

\begin{tabular}{lccccccl}
\hline \multicolumn{1}{c}{ Field Name } & $\begin{array}{c}\text { Data } \\
\text { Type }\end{array}$ & $\begin{array}{c}\text { Field } \\
\text { Length }\end{array}$ & $\begin{array}{c}\text { Decimal } \\
\text { Places }\end{array}$ & $\begin{array}{c}\text { Required } \\
\text { Field }\end{array}$ & $\begin{array}{c}\text { Primary } \\
\text { Key }\end{array}$ & Definition \\
\hline PhotoQuality & varchar & 50 & 0 & $\mathrm{~N}$ & $\mathrm{~N}$ & Description of photo quality. \\
PhotoQualityID & int & 0 & 0 & $\mathrm{Y}$ & $\mathrm{Y}$ & PhotoQuality record identifier. \\
\hline
\end{tabular}

luPhotoType

\begin{tabular}{lccccccl}
\hline \multicolumn{1}{c}{ Field Name } & $\begin{array}{c}\text { Data } \\
\text { Type }\end{array}$ & $\begin{array}{c}\text { Field } \\
\text { Length }\end{array}$ & $\begin{array}{c}\text { Decimal } \\
\text { Places }\end{array}$ & $\begin{array}{c}\text { Required } \\
\text { Field }\end{array}$ & $\begin{array}{c}\text { Primary } \\
\text { Key }\end{array}$ & Definition \\
\hline PhotoType & varchar & 50 & 0 & $\mathrm{Y}$ & $\mathrm{N}$ & Description of physical storage media of photo. \\
PhotoTypeID & int & 0 & 0 & $\mathrm{Y}$ & $\mathrm{Y}$ & PhotoType record identifier. \\
\hline
\end{tabular}

\section{PlantEnhancement Table Look Ups}

lulrrigationMethods

\begin{tabular}{lcccccl}
\hline \multicolumn{1}{c}{ Field Name } & $\begin{array}{c}\text { Data } \\
\text { Type }\end{array}$ & $\begin{array}{c}\text { Field } \\
\text { Length }\end{array}$ & $\begin{array}{c}\text { Decimal } \\
\text { Places }\end{array}$ & $\begin{array}{c}\text { Required } \\
\text { Field }\end{array}$ & $\begin{array}{c}\text { Primary } \\
\text { Key }\end{array}$ & Definition \\
\hline Description & varchar & 255 & 0 & $\mathrm{~N}$ & $\mathrm{~N}$ & Description of the irrigation method. \\
IrrigationMethod & varchar & 25 & 0 & $\mathrm{Y}$ & $\mathrm{N}$ & Method used to water plantings. \\
IrrigationMethodID & int & 0 & 0 & $\mathrm{Y}$ & $\mathrm{Y}$ & IrrigationMethod record identifier. \\
\hline
\end{tabular}

luPlantingForms

\begin{tabular}{lcccccl}
\hline \multicolumn{1}{c}{ Field Name } & $\begin{array}{c}\text { Data } \\
\text { Type }\end{array}$ & $\begin{array}{c}\text { Field } \\
\text { Length }\end{array}$ & $\begin{array}{c}\text { Decimal } \\
\text { Places }\end{array}$ & $\begin{array}{c}\text { Required } \\
\text { Field }\end{array}$ & $\begin{array}{c}\text { Primary } \\
\text { Key }\end{array}$ & Definition \\
\hline Description & varchar & 255 & 0 & $\mathrm{~N}$ & $\mathrm{~N}$ & Description of the planting form. \\
PlantingForm & varchar & 25 & 0 & $\mathrm{Y}$ & $\mathrm{N}$ & Form of the plant being planted. \\
PlantingFormID & int & 0 & 0 & $\mathrm{Y}$ & $\mathrm{Y}$ & PlantingForm record identifier. \\
\hline
\end{tabular}

\section{PlantHeirarchy Table Look Ups}

luHeightClass

\begin{tabular}{lccccccc}
\hline Field Name & $\begin{array}{c}\text { Data } \\
\text { Type }\end{array}$ & $\begin{array}{c}\text { Field } \\
\text { Length }\end{array}$ & $\begin{array}{c}\text { Decimal } \\
\text { Places }\end{array}$ & $\begin{array}{c}\text { Required } \\
\text { Field }\end{array}$ & $\begin{array}{c}\text { Primary } \\
\text { Key }\end{array}$ & Definition \\
\hline HeightClass & varchar & 15 & 0 & Y & Y & Height class of the plant. & \\
\hline
\end{tabular}

\section{PlantObservationDetails Table Look Ups}

luAgeMetric

\begin{tabular}{|c|c|c|c|c|c|c|}
\hline $\begin{array}{l}\text { Field } \\
\text { Name }\end{array}$ & $\begin{array}{l}\text { Data } \\
\text { Type }\end{array}$ & $\begin{array}{c}\text { Field } \\
\text { Length }\end{array}$ & $\begin{array}{c}\text { Decimal } \\
\text { Places }\end{array}$ & $\begin{array}{l}\text { Required } \\
\text { Field }\end{array}$ & $\begin{array}{c}\text { Primary } \\
\text { Key }\end{array}$ & Definition \\
\hline AgeMetric & varchar & 25 & 0 & $\mathrm{Y}$ & $\mathrm{Y}$ & Metric used to measure plant age (years, months, days). \\
\hline \multicolumn{7}{|c|}{ luEstMethod } \\
\hline $\begin{array}{l}\text { Field } \\
\text { Name }\end{array}$ & $\begin{array}{l}\text { Data } \\
\text { Type }\end{array}$ & $\begin{array}{c}\text { Field } \\
\text { Length }\end{array}$ & $\begin{array}{l}\text { Decimal } \\
\text { Places }\end{array}$ & $\begin{array}{l}\text { Required } \\
\text { Field }\end{array}$ & $\begin{array}{c}\text { Primary } \\
\text { Key }\end{array}$ & Definition \\
\hline
\end{tabular}


luDensityUnit

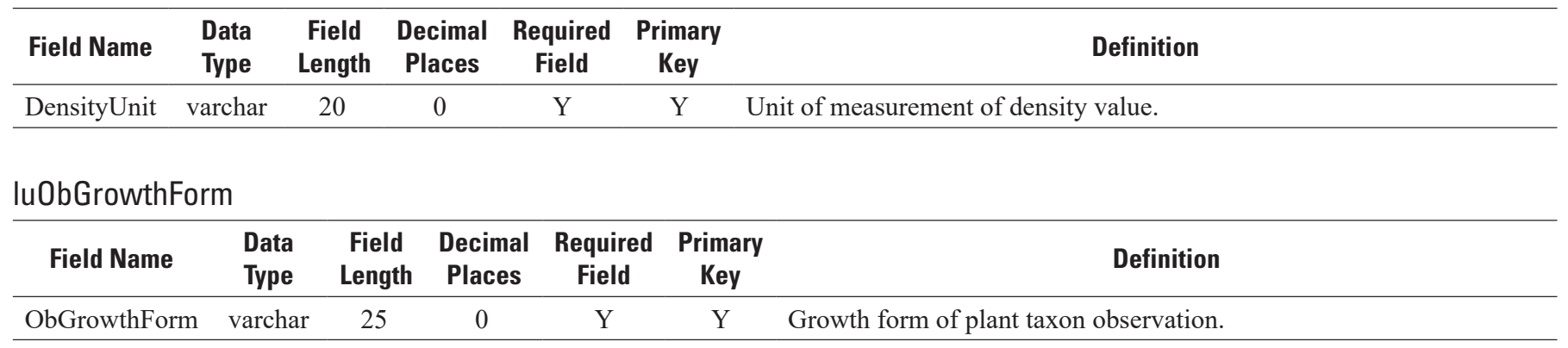

luPlantObDetailType

\begin{tabular}{lccccccc}
\hline Field Name & $\begin{array}{c}\text { Data } \\
\text { Type }\end{array}$ & $\begin{array}{c}\text { Field } \\
\text { Length }\end{array}$ & $\begin{array}{c}\text { Decimal } \\
\text { Places }\end{array}$ & $\begin{array}{c}\text { Required } \\
\text { Field }\end{array}$ & $\begin{array}{c}\text { Primary } \\
\text { Key }\end{array}$ & Definition \\
\hline Description & varchar & 100 & 0 & $\mathrm{~N}$ & $\mathrm{~N}$ & Description of the detail type. \\
DetailType & varchar & 20 & 0 & $\mathrm{Y}$ & $\mathrm{Y}$ & $\begin{array}{c}\text { Describes the context of the plant observation, such as individual or patch/ } \\
\text { stand. }\end{array}$ \\
\hline
\end{tabular}

luPlantSizeClass

\begin{tabular}{cccccccc}
\hline $\begin{array}{c}\text { Field } \\
\text { Name }\end{array}$ & $\begin{array}{c}\text { Data } \\
\text { Type }\end{array}$ & $\begin{array}{c}\text { Field } \\
\text { Length }\end{array}$ & $\begin{array}{c}\text { Decimal } \\
\text { Places }\end{array}$ & $\begin{array}{c}\text { Required } \\
\text { Field }\end{array}$ & $\begin{array}{c}\text { Primary } \\
\text { Key }\end{array}$ & Definition \\
\hline SizeClass & int & 0 & 0 & Y & Y & Size class of plant in cm. & \\
\hline
\end{tabular}

\section{ProjectID Table Look Ups}

luProjectCategory

\begin{tabular}{lcccccl}
\hline \multicolumn{1}{c}{ Field Name } & $\begin{array}{c}\text { Data } \\
\text { Type }\end{array}$ & $\begin{array}{c}\text { Field } \\
\text { Length }\end{array}$ & $\begin{array}{c}\text { Decimal } \\
\text { Places }\end{array}$ & $\begin{array}{c}\text { Required } \\
\text { Field }\end{array}$ & $\begin{array}{c}\text { Primary } \\
\text { Key }\end{array}$ & Definition \\
\hline Description & varchar & 100 & 0 & $\mathrm{~N}$ & $\mathrm{~N}$ & Description of the category. \\
ProjectCategory & varchar & 25 & 0 & $\mathrm{Y}$ & $\mathrm{Y}$ & Category of project being conducted. \\
\hline
\end{tabular}

\section{Refugia Table Look Ups}

luRefugiaContext

\begin{tabular}{ccccccc}
\hline $\begin{array}{c}\text { Field } \\
\text { Name }\end{array}$ & $\begin{array}{c}\text { Data } \\
\text { Type }\end{array}$ & $\begin{array}{c}\text { Field } \\
\text { Length }\end{array}$ & $\begin{array}{c}\text { Decimal } \\
\text { Places }\end{array}$ & $\begin{array}{c}\text { Required } \\
\text { Field }\end{array}$ & $\begin{array}{c}\text { Primary } \\
\text { Key }\end{array}$ & Definition \\
\hline Context & varchar & 20 & 0 & Y & Y & Type of environment within the site where refugium is located. \\
\hline
\end{tabular}

luRefugiaType

\begin{tabular}{lcccccc}
\hline Field Name & $\begin{array}{c}\text { Data } \\
\text { Type }\end{array}$ & $\begin{array}{c}\text { Field } \\
\text { Length }\end{array}$ & $\begin{array}{c}\text { Decimal } \\
\text { Places }\end{array}$ & $\begin{array}{c}\text { Required } \\
\text { Field }\end{array}$ & $\begin{array}{c}\text { Primary } \\
\text { Key }\end{array}$ & Definition \\
\hline RefugiaType & varchar & 20 & 0 & Y & Y & $\begin{array}{c}\text { Any material that provides shelter, cover, or hiding place, (for example, } \\
\text { rocks, rock crevices, woody debris). }\end{array}$ \\
\hline
\end{tabular}




\section{RoadsTrails Table Look Ups}

luFreqofTravel

\begin{tabular}{ccrccccl}
\hline Field Name & $\begin{array}{c}\text { Data } \\
\text { Type }\end{array}$ & $\begin{array}{c}\text { Field } \\
\text { Length }\end{array}$ & $\begin{array}{c}\text { Decimal } \\
\text { Places }\end{array}$ & $\begin{array}{c}\text { Required } \\
\text { Field }\end{array}$ & $\begin{array}{c}\text { Primary } \\
\text { Key }\end{array}$ & Definition \\
\hline FrequencyTraveled & varchar & 30 & 0 & $\mathrm{Y}$ & $\mathrm{Y}$ & How often the road/trail is used, likely estimated. \\
\hline
\end{tabular}

luRoadsTrailsAccess

\begin{tabular}{ccccccc}
\hline Field Name & $\begin{array}{c}\text { Data } \\
\text { Type }\end{array}$ & $\begin{array}{c}\text { Field } \\
\text { Length }\end{array}$ & $\begin{array}{c}\text { Decimal } \\
\text { Places }\end{array}$ & $\begin{array}{c}\text { Required } \\
\text { Field }\end{array}$ & $\begin{array}{c}\text { Primary } \\
\text { Key }\end{array}$ & Definition \\
\hline RoadTrailsAccess & varchar & 30 & 0 & $\mathrm{Y}$ & $\mathrm{Y}$ & Description of how and why road/trail is used.
\end{tabular}

luRoadsTrailsEdge

\begin{tabular}{lcccccc}
\hline $\begin{array}{c}\text { Field } \\
\text { Name }\end{array}$ & $\begin{array}{c}\text { Data } \\
\text { Type }\end{array}$ & $\begin{array}{c}\text { Field } \\
\text { Length }\end{array}$ & $\begin{array}{c}\text { Decimal } \\
\text { Places }\end{array}$ & $\begin{array}{c}\text { Required } \\
\text { Field }\end{array}$ & $\begin{array}{c}\text { Primary } \\
\text { Key }\end{array}$ & Definition \\
\hline Edge & varchar & 30 & 0 & Y & Y & Composition of interface between the road/trail and the site. \\
\hline
\end{tabular}

luRoadsTrailsType

\begin{tabular}{lcccccc}
\hline $\begin{array}{c}\text { Field } \\
\text { Name }\end{array}$ & $\begin{array}{c}\text { Data } \\
\text { Type }\end{array}$ & $\begin{array}{c}\text { Field } \\
\text { Length }\end{array}$ & $\begin{array}{c}\text { Decimal } \\
\text { Places }\end{array}$ & $\begin{array}{c}\text { Required } \\
\text { Field }\end{array}$ & $\begin{array}{c}\text { Primary } \\
\text { Key }\end{array}$ & Definition \\
\hline Type & varchar & 30 & 0 & Y & Y & General type and construction of road/trail. \\
\hline
\end{tabular}

\section{Site Table Look Ups}

luCounty

\begin{tabular}{lcccccl}
\hline \multicolumn{1}{c}{ Field Name } & $\begin{array}{c}\text { Data } \\
\text { Type }\end{array}$ & $\begin{array}{c}\text { Field } \\
\text { Length }\end{array}$ & $\begin{array}{c}\text { Decimal } \\
\text { Places }\end{array}$ & $\begin{array}{c}\text { Required } \\
\text { Field }\end{array}$ & $\begin{array}{c}\text { Primary } \\
\text { Key }\end{array}$ & Definition \\
\hline County & varchar & 50 & 0 & $\mathrm{Y}$ & $\mathrm{N}$ & County Name. \\
CountyAbbreviation & char & 3 & 0 & $\mathrm{Y}$ & $\mathrm{N}$ & Three letter abbreviation of county name. \\
CountyFIPSCode & smallint & 5 & 0 & $\mathrm{~N}$ & $\mathrm{~N}$ & Federal Information Processing Standard (FIPS) code of county. \\
CountyID & smallint & 5 & 0 & $\mathrm{Y}$ & $\mathrm{Y}$ & County record identifier. \\
StateCode & char & 2 & 0 & $\mathrm{~N}$ & $\mathrm{~N}$ & Two letter State Code (abbreviation). \\
\hline
\end{tabular}

luSiteQuality

\begin{tabular}{lcccccl}
\hline \multicolumn{1}{c}{ Field Name } & $\begin{array}{c}\text { Data } \\
\text { Type }\end{array}$ & $\begin{array}{c}\text { Field } \\
\text { Length }\end{array}$ & $\begin{array}{c}\text { Decimal } \\
\text { Places }\end{array}$ & $\begin{array}{c}\text { Required } \\
\text { Field }\end{array}$ & $\begin{array}{c}\text { Primary } \\
\text { Key }\end{array}$ & Definition \\
\hline SiteQuality & varchar & 50 & 0 & $\mathrm{Y}$ & $\mathrm{N}$ & Overall quality of site. \\
SiteQualityDescription & varchar & 350 & 0 & $\mathrm{~N}$ & $\mathrm{~N}$ & Description of the site quality. \\
SiteQualityID & int & 0 & 0 & $\mathrm{Y}$ & $\mathrm{Y}$ & SiteQuality record identifier. \\
\hline
\end{tabular}

\section{Site_Site Table Look Ups}

luSiteRelationshipType

\begin{tabular}{ccccccc}
\hline Field Name & $\begin{array}{c}\text { Data } \\
\text { Type }\end{array}$ & $\begin{array}{c}\text { Field } \\
\text { Length }\end{array}$ & $\begin{array}{c}\text { Decimal } \\
\text { Places }\end{array}$ & $\begin{array}{c}\text { Required } \\
\text { Field }\end{array}$ & $\begin{array}{c}\text { Primary } \\
\text { Key }\end{array}$ & Definition \\
\hline RelationshipType & varchar & 20 & 0 & Y & Y & Identifies the relationship between the parent and child site. \\
\hline
\end{tabular}


Site_SiteThreatType Table Look Ups

luSiteThreatType

\begin{tabular}{lcrcccl}
\hline \multicolumn{1}{c}{ Field Name } & $\begin{array}{c}\text { Data } \\
\text { Type }\end{array}$ & $\begin{array}{c}\text { Field } \\
\text { Length }\end{array}$ & $\begin{array}{c}\text { Decimal } \\
\text { Places }\end{array}$ & $\begin{array}{c}\text { Required } \\
\text { Field }\end{array}$ & $\begin{array}{c}\text { Primary } \\
\text { Key }\end{array}$ & Definition \\
\hline Description & varchar & 255 & 0 & $\mathrm{~N}$ & $\mathrm{~N}$ & Description of the threat. \\
SiteThreatType & varchar & 50 & 0 & $\mathrm{Y}$ & $\mathrm{N}$ & Threat to the site. \\
SiteThreatTypeID & int & 0 & 0 & $\mathrm{Y}$ & $\mathrm{Y}$ & SiteThreatType record identifier. \\
\hline
\end{tabular}

\section{SoilPhysicalObservation Table Look Ups}

luSoilMethod

\begin{tabular}{cccccccc}
\hline $\begin{array}{c}\text { Field } \\
\text { Name }\end{array}$ & $\begin{array}{c}\text { Data } \\
\text { Type }\end{array}$ & $\begin{array}{c}\text { Field } \\
\text { Length }\end{array}$ & $\begin{array}{c}\text { Decimal } \\
\text { Places }\end{array}$ & $\begin{array}{c}\text { Required } \\
\text { Field }\end{array}$ & $\begin{array}{c}\text { Primary } \\
\text { Key }\end{array}$ & Definition \\
\hline Method & varchar & 30 & 0 & Y & Y & Method used to analyze the soil. & \\
\hline
\end{tabular}

luSoilTextureClass

\begin{tabular}{lcccccc}
\hline Field Name & $\begin{array}{c}\text { Data } \\
\text { Type }\end{array}$ & $\begin{array}{c}\text { Field } \\
\text { Length }\end{array}$ & $\begin{array}{c}\text { Decimal } \\
\text { Places }\end{array}$ & $\begin{array}{c}\text { Required } \\
\text { Field }\end{array}$ & $\begin{array}{c}\text { Primary } \\
\text { Key }\end{array}$ & Definition \\
\hline TextureClass & varchar & 20 & 0 & Y & Y & Texture classification of soil/soil sample. \\
\hline
\end{tabular}

luSoilUnit

\begin{tabular}{lcccccc}
\hline $\begin{array}{c}\text { Field } \\
\text { Name }\end{array}$ & $\begin{array}{c}\text { Data } \\
\text { Type }\end{array}$ & $\begin{array}{c}\text { Field } \\
\text { Length }\end{array}$ & $\begin{array}{c}\text { Decimal } \\
\text { Places }\end{array}$ & $\begin{array}{c}\text { Required } \\
\text { Field }\end{array}$ & $\begin{array}{c}\text { Primary } \\
\text { Key }\end{array}$ & Definition \\
\hline Unit & varchar & 20 & 0 & Y & Y & Unit of measurement of soil sample. \\
\hline
\end{tabular}

\section{SourceXY Table Look Ups}

luXYCoordType

\begin{tabular}{lcccccl}
\hline \multicolumn{1}{c}{ Field Name } & $\begin{array}{c}\text { Data } \\
\text { Type }\end{array}$ & $\begin{array}{c}\text { Field } \\
\text { Length }\end{array}$ & $\begin{array}{c}\text { Decimal } \\
\text { Places }\end{array}$ & $\begin{array}{c}\text { Required } \\
\text { Field }\end{array}$ & $\begin{array}{c}\text { Primary } \\
\text { Key }\end{array}$ & Definition \\
\hline Description & varchar & 255 & 0 & $\mathrm{~N}$ & $\mathrm{~N}$ & Description of the Coordinate Type. \\
XYCoordType & varchar & 30 & 0 & $\mathrm{Y}$ & $\mathrm{N}$ & Name of coordinate system used. \\
XYCoordTypeID & int & 0 & 0 & $\mathrm{Y}$ & $\mathrm{Y}$ & XYCoordType record identifier. \\
\hline
\end{tabular}

\section{SpatialFeature_CLDB Table Look Ups}

luSFCLDBMethod

\begin{tabular}{ccccccc}
\hline $\begin{array}{c}\text { Field } \\
\text { Name }\end{array}$ & $\begin{array}{c}\text { Data } \\
\text { Type }\end{array}$ & $\begin{array}{c}\text { Field } \\
\text { Length }\end{array}$ & $\begin{array}{c}\text { Decimal } \\
\text { Places }\end{array}$ & $\begin{array}{c}\text { Required } \\
\text { Field }\end{array}$ & $\begin{array}{c}\text { Primary } \\
\text { Key }\end{array}$ & Definition \\
\hline Method & varchar & 30 & 0 & $\mathrm{Y}$ & $\mathrm{Y}$ & $\begin{array}{c}\text { The method by which the association between SpatialFeatureID and LandID } \\
\text { was made. }\end{array}$ \\
\hline
\end{tabular}




\section{SpeciesHabitatFeature Table Look Ups}

luFeatureType

\begin{tabular}{ccccccc}
\hline Field Name & $\begin{array}{c}\text { Data } \\
\text { Type }\end{array}$ & $\begin{array}{c}\text { Field } \\
\text { Length }\end{array}$ & $\begin{array}{c}\text { Decimal } \\
\text { Places }\end{array}$ & $\begin{array}{c}\text { Required } \\
\text { Field }\end{array}$ & $\begin{array}{c}\text { Primary } \\
\text { Key }\end{array}$ & Definition \\
\hline FeatureType & varchar & 20 & 0 & Y & Y & Type of feature being used by observed species. \\
\hline
\end{tabular}

luHabSuitability

\begin{tabular}{lccccccl}
\hline $\begin{array}{c}\text { Field } \\
\text { Name }\end{array}$ & $\begin{array}{c}\text { Data } \\
\text { Type }\end{array}$ & $\begin{array}{c}\text { Field } \\
\text { Length }\end{array}$ & $\begin{array}{c}\text { Decimal } \\
\text { Places }\end{array}$ & $\begin{array}{c}\text { Required } \\
\text { Field }\end{array}$ & $\begin{array}{c}\text { Primary } \\
\text { Key }\end{array}$ & Definition \\
\hline HSID & char & 1 & 0 & $\mathrm{Y}$ & $\mathrm{Y}$ & HabitatSuitability record identifier. \\
Suitability & varchar & 15 & 0 & $\mathrm{Y}$ & $\mathrm{N}$ & Suitability of habitat for species. \\
\hline
\end{tabular}

luRelativeAbundance

\begin{tabular}{ccccccc}
\hline Field Name & $\begin{array}{c}\text { Data } \\
\text { Type }\end{array}$ & $\begin{array}{c}\text { Field } \\
\text { Length }\end{array}$ & $\begin{array}{c}\text { Decimal } \\
\text { Places }\end{array}$ & $\begin{array}{c}\text { Required } \\
\text { Field }\end{array}$ & $\begin{array}{c}\text { Primary } \\
\text { Key }\end{array}$ & Definition \\
\hline RelativeAbundance & varchar & 10 & 0 & $\mathrm{Y}$ & $\mathrm{Y}$ & Relative amount of feature observed. \\
\hline
\end{tabular}

\section{StudyPersonnel Table Look Ups}

luAffiliateRole

\begin{tabular}{lcccccc}
\hline Field Name & $\begin{array}{c}\text { Data } \\
\text { Type }\end{array}$ & $\begin{array}{c}\text { Field } \\
\text { Length }\end{array}$ & $\begin{array}{c}\text { Decimal } \\
\text { Places }\end{array}$ & $\begin{array}{c}\text { Required } \\
\text { Field }\end{array}$ & $\begin{array}{c}\text { Primary } \\
\text { Key }\end{array}$ & Definition \\
\hline AffiliateRole & varchar & 50 & 0 & $\mathrm{Y}$ & $\mathrm{Y}$ & Role of person associated with this survey. \\
Description & varchar & 255 & 0 & $\mathrm{~N}$ & $\mathrm{~N}$ & Description of the affiliate role. \\
\hline
\end{tabular}

\section{SurveyDescription Table Look Ups}

luStudyType

\begin{tabular}{cccccccl}
\hline $\begin{array}{c}\text { Field } \\
\text { Name }\end{array}$ & $\begin{array}{c}\text { Data } \\
\text { Type }\end{array}$ & $\begin{array}{c}\text { Field } \\
\text { Length }\end{array}$ & $\begin{array}{c}\text { Decimal } \\
\text { Places }\end{array}$ & $\begin{array}{c}\text { Required } \\
\text { Field }\end{array}$ & $\begin{array}{c}\text { Primary } \\
\text { Key }\end{array}$ & Definition \\
\hline StudyType & varchar & 40 & 0 & Y & Y & Type of study conducted. & \\
\hline
\end{tabular}

luSourceType

\begin{tabular}{lccccccl}
\hline \multicolumn{1}{c}{ Field Name } & $\begin{array}{c}\text { Data } \\
\text { Type }\end{array}$ & $\begin{array}{c}\text { Field } \\
\text { Length }\end{array}$ & $\begin{array}{c}\text { Decimal } \\
\text { Places }\end{array}$ & $\begin{array}{c}\text { Required } \\
\text { Field }\end{array}$ & $\begin{array}{c}\text { Primary } \\
\text { Key }\end{array}$ & Definition \\
\hline Description & varchar & 75 & 0 & $\mathrm{~N}$ & $\mathrm{~N}$ & Distinguishes the original source of the data. \\
SourceTypeID & int & 0 & 0 & $\mathrm{Y}$ & $\mathrm{Y}$ & SourceType record identifier. \\
\hline
\end{tabular}

\section{SurveyDescription_Site Table Look Ups}

luMSCPLandDesignation

\begin{tabular}{ccccccc}
\hline Field Name & $\begin{array}{c}\text { Data } \\
\text { Type }\end{array}$ & $\begin{array}{c}\text { Field } \\
\text { Length }\end{array}$ & $\begin{array}{c}\text { Decimal } \\
\text { Places }\end{array}$ & $\begin{array}{c}\text { Required } \\
\text { Field }\end{array}$ & $\begin{array}{c}\text { Primary } \\
\text { Key }\end{array}$ & Definition \\
\hline MSCPLandDesignation & varchar & 10 & 0 & Y & Y & $\begin{array}{c}\text { Designation of the land in the Multiple Species Conservation } \\
\text { Plan (MSCP). }\end{array}$ \\
\hline
\end{tabular}




\section{SurveyEvent Table Look Ups}

ConstrainBy

\begin{tabular}{lccccccl}
\hline \multicolumn{1}{c}{ Field Name } & $\begin{array}{c}\text { Data } \\
\text { Type }\end{array}$ & $\begin{array}{c}\text { Field } \\
\text { Length }\end{array}$ & $\begin{array}{c}\text { Decimal } \\
\text { Places }\end{array}$ & $\begin{array}{c}\text { Required } \\
\text { Field }\end{array}$ & $\begin{array}{c}\text { Primary } \\
\text { Key }\end{array}$ & Definition \\
\hline ConstrainedBy & char & 4 & 0 & $\mathrm{Y}$ & $\mathrm{Y}$ & Metric by which survey was constrained. \\
ConstraintDefinition & varchar & 50 & 0 & $\mathrm{Y}$ & $\mathrm{N}$ & Definition of constraint. \\
\hline
\end{tabular}

luSurveyableAreaUnit

\begin{tabular}{ccccccc}
\hline $\begin{array}{c}\text { Field } \\
\text { Name }\end{array}$ & $\begin{array}{c}\text { Data } \\
\text { Type }\end{array}$ & $\begin{array}{c}\text { Field } \\
\text { Length }\end{array}$ & $\begin{array}{c}\text { Decimal } \\
\text { Places }\end{array}$ & $\begin{array}{c}\text { Required } \\
\text { Field }\end{array}$ & $\begin{array}{c}\text { Primary } \\
\text { Key }\end{array}$ & Definition \\
\hline SAUnit & varchar & 20 & 0 & Y & Y & Unit used for surveyable area determination. \\
\hline
\end{tabular}

luStudyType

\begin{tabular}{cccccccl}
\hline $\begin{array}{c}\text { Field } \\
\text { Name }\end{array}$ & $\begin{array}{c}\text { Data } \\
\text { Type }\end{array}$ & $\begin{array}{c}\text { Field } \\
\text { Length }\end{array}$ & $\begin{array}{c}\text { Decimal } \\
\text { Places }\end{array}$ & $\begin{array}{c}\text { Required } \\
\text { Field }\end{array}$ & $\begin{array}{c}\text { Primary } \\
\text { Key }\end{array}$ & Definition \\
\hline StudyType & varchar & 40 & 0 & Y & Y & Type of study conducted. & \\
\hline
\end{tabular}

UnitEffort

\begin{tabular}{lccccccl}
\hline Field Name & $\begin{array}{c}\text { Data } \\
\text { Type }\end{array}$ & $\begin{array}{c}\text { Field } \\
\text { Length }\end{array}$ & $\begin{array}{c}\text { Decimal } \\
\text { Places }\end{array}$ & $\begin{array}{c}\text { Required } \\
\text { Field }\end{array}$ & $\begin{array}{c}\text { Primary } \\
\text { Key }\end{array}$ & Definition \\
\hline UnitEffort & varchar & 30 & 0 & $\mathrm{Y}$ & $\mathrm{N}$ & Unit effort of the survey. & \\
UnitEffortID & char & 2 & 0 & $\mathrm{Y}$ & $\mathrm{Y}$ & UnitEffort record identifier. & \\
\hline
\end{tabular}

\section{SurveyEvent_Affiliate Table Look Ups}

luAffiliateRole

\begin{tabular}{lccccccl}
\hline Field Name & $\begin{array}{c}\text { Data } \\
\text { Type }\end{array}$ & $\begin{array}{c}\text { Field } \\
\text { Length }\end{array}$ & $\begin{array}{c}\text { Decimal } \\
\text { Places }\end{array}$ & $\begin{array}{c}\text { Required } \\
\text { Field }\end{array}$ & $\begin{array}{c}\text { Primary } \\
\text { Key }\end{array}$ & Definition \\
\hline AffiliateRole & varchar & 50 & 0 & $\mathrm{Y}$ & $\mathrm{Y}$ & Role of person associated with this survey. \\
Description & varchar & 255 & 0 & $\mathrm{~N}$ & $\mathrm{~N}$ & Description of the affiliate role. \\
\hline
\end{tabular}

\section{SurveyEvent_Site Table Look Ups}

luAmtSurveyedUnit

\begin{tabular}{ccccccc}
\hline Field Name & $\begin{array}{c}\text { Data } \\
\text { Type }\end{array}$ & $\begin{array}{c}\text { Field } \\
\text { Length }\end{array}$ & $\begin{array}{c}\text { Decimal } \\
\text { Places }\end{array}$ & $\begin{array}{c}\text { Required } \\
\text { Field }\end{array}$ & $\begin{array}{c}\text { Primary } \\
\text { Key }\end{array}$ & Definition \\
\hline AmountSurveyedUnit & varchar & 15 & 0 & $\mathrm{Y}$ & $\mathrm{Y}$ & $\begin{array}{c}\text { Unit of measurement used to quantify the AmountSurveyed } \\
\text { value. }\end{array}$ \\
\hline
\end{tabular}

\section{SurveyEvent_SpatialFeature Table Look Ups}

IuSESFRelationship

\begin{tabular}{ccccccc}
\hline Field Name & $\begin{array}{c}\text { Data } \\
\text { Type }\end{array}$ & $\begin{array}{c}\text { Field } \\
\text { Length }\end{array}$ & $\begin{array}{c}\text { Decimal } \\
\text { Places }\end{array}$ & $\begin{array}{c}\text { Required } \\
\text { Field }\end{array}$ & $\begin{array}{c}\text { Primary } \\
\text { Key }\end{array}$ & Definition \\
\hline Relationship & varchar & 20 & 0 & Y & Y & $\begin{array}{r}\text { Identifies the relationship between the SurveyEvent record and an } \\
\text { associated SpatialFeature record (actual start/end, and so forth). }\end{array}$ \\
\hline
\end{tabular}




\section{SurveyMethod Table Look Ups}

luSurveyMethod

\begin{tabular}{lccccccl}
\hline \multirow{2}{*}{ Field Name } & $\begin{array}{c}\text { Data } \\
\text { Type }\end{array}$ & $\begin{array}{c}\text { Field } \\
\text { Length }\end{array}$ & $\begin{array}{c}\text { Decimal } \\
\text { Places }\end{array}$ & $\begin{array}{c}\text { Required } \\
\text { Field }\end{array}$ & $\begin{array}{c}\text { Primary } \\
\text { Key }\end{array}$ & Definition \\
\hline Description & varchar & 250 & 0 & $\mathrm{~N}$ & $\mathrm{~N}$ & Description of the survey method. \\
SurveyMethod & varchar & 30 & 0 & $\mathrm{Y}$ & $\mathrm{Y}$ & Method used to detect a taxon during the survey. \\
\hline
\end{tabular}

\section{TaxaObservation Table Look Ups}

luAbundanceCategory

\begin{tabular}{|c|c|c|c|c|c|c|}
\hline Field Name & $\begin{array}{l}\text { Data } \\
\text { Type }\end{array}$ & $\begin{array}{l}\text { Field } \\
\text { Length }\end{array}$ & $\begin{array}{l}\text { Decimal } \\
\text { Places }\end{array}$ & $\begin{array}{l}\text { Required } \\
\text { Field }\end{array}$ & $\begin{array}{l}\text { Primary } \\
\text { Key }\end{array}$ & Definition \\
\hline AbundanceCategory & varchar & 50 & 0 & $\mathrm{Y}$ & $\mathrm{N}$ & $\begin{array}{l}\text { Abundance category for a group of plant specimens based } \\
\text { on spatial presence. }\end{array}$ \\
\hline AbundanceCategoryCode & varchar & 2 & 0 & Y & $\mathrm{Y}$ & Abbreviation of abundance category. \\
\hline Description & varchar & 255 & 0 & $\mathrm{~N}$ & $\mathrm{~N}$ & Description of the abundance category. \\
\hline
\end{tabular}

luHistoricalPresence

\begin{tabular}{ccccccc}
\hline Field Name & $\begin{array}{c}\text { Data } \\
\text { Type }\end{array}$ & $\begin{array}{c}\text { Field } \\
\text { Length }\end{array}$ & $\begin{array}{c}\text { Decimal } \\
\text { Places }\end{array}$ & $\begin{array}{c}\text { Required } \\
\text { Field }\end{array}$ & $\begin{array}{c}\text { Primary } \\
\text { Key }\end{array}$ & Definition \\
\hline HistoricalPresence & varchar & 10 & 0 & Y & Y & $\begin{array}{c}\text { Status of species at this location regarding whether it is considered } \\
\text { native, exotic, or invasive. }\end{array}$ \\
\hline
\end{tabular}

\section{luRemovallntrodutionValues}

\begin{tabular}{lcccccl}
\hline \multicolumn{1}{c}{ Field Name } & $\begin{array}{c}\text { Data } \\
\text { Type }\end{array}$ & $\begin{array}{c}\text { Field } \\
\text { Length }\end{array}$ & $\begin{array}{c}\text { Decimal } \\
\text { Places }\end{array}$ & $\begin{array}{c}\text { Required } \\
\text { Field }\end{array}$ & $\begin{array}{c}\text { Primary } \\
\text { Key }\end{array}$ & Definition \\
\hline Description & varchar & 255 & 0 & $\mathrm{~N}$ & $\mathrm{~N}$ & Description of the removal or introduction value. \\
RemovalIntroValue & varchar & 25 & 0 & $\mathrm{Y}$ & $\mathrm{N}$ & Method used to remove or introduce a species into the habitat. \\
RemovalIntroValueID & int & 0 & 0 & $\mathrm{Y}$ & $\mathrm{Y}$ & RemovalIntroductionValue record identifier. \\
\hline
\end{tabular}

IuSpeciesDetected

\begin{tabular}{lccccccl}
\hline Field Name & $\begin{array}{c}\text { Data } \\
\text { Type }\end{array}$ & $\begin{array}{c}\text { Field } \\
\text { Length }\end{array}$ & $\begin{array}{c}\text { Decimal } \\
\text { Places }\end{array}$ & $\begin{array}{c}\text { Required } \\
\text { Field }\end{array}$ & $\begin{array}{c}\text { Primary } \\
\text { Key }\end{array}$ & Definition \\
\hline Description & varchar & 20 & 0 & $\mathrm{~N}$ & $\mathrm{~N}$ & Whether a species was detected/not detected/not surveyed for. \\
SDCode & numeric & 1 & 0 & $\mathrm{Y}$ & $\mathrm{Y}$ & luSpeciesDetected code record identifier. \\
\hline
\end{tabular}

luTaxaRole

\begin{tabular}{|c|c|c|c|c|c|c|}
\hline Field Name & $\begin{array}{l}\text { Data } \\
\text { Type }\end{array}$ & $\begin{array}{c}\text { Field } \\
\text { Length }\end{array}$ & $\begin{array}{c}\text { Decimal } \\
\text { Places }\end{array}$ & $\begin{array}{l}\text { Required } \\
\text { Field }\end{array}$ & $\begin{array}{c}\text { Primary } \\
\text { Key }\end{array}$ & Definition \\
\hline Description & varchar & 100 & 0 & $\mathrm{~N}$ & $\mathrm{~N}$ & Description of the taxon's role. \\
\hline TaxaRoleCode & varchar & 2 & 0 & $\mathrm{~N}$ & $\mathrm{~N}$ & Code associated with taxa role. \\
\hline TaxaRoleID & int & 0 & 0 & $\mathrm{Y}$ & $\mathrm{Y}$ & TaxaRole record identifier. \\
\hline
\end{tabular}


Taxa

\begin{tabular}{|c|c|c|c|c|c|c|}
\hline Field Name & $\begin{array}{l}\text { Data } \\
\text { Type }\end{array}$ & $\begin{array}{c}\text { Field } \\
\text { Length }\end{array}$ & $\begin{array}{c}\text { Decimal } \\
\text { Places }\end{array}$ & $\begin{array}{l}\text { Required } \\
\text { Field }\end{array}$ & $\begin{array}{c}\text { Primary } \\
\text { Key }\end{array}$ & Definition \\
\hline Abbreviation & varchar & 10 & 0 & $\mathrm{~N}$ & $\mathrm{~N}$ & Taxon abbreviation. \\
\hline AuthorInfraSpecies & varchar & 80 & 0 & $\mathrm{~N}$ & $\mathrm{~N}$ & Author of the infraspecific name. \\
\hline Code1 & varchar & 50 & 0 & $\mathrm{~N}$ & $\mathrm{~N}$ & Taxon species code. \\
\hline CreatedDate & datetime & 0 & 0 & Y & $\mathrm{N}$ & Date and time when record was created. \\
\hline EditDate & datetime & 0 & 0 & $\mathrm{~N}$ & $\mathrm{~N}$ & Date and time when record was most recently updated. \\
\hline EditedBy & varchar & 64 & 0 & $\mathrm{~N}$ & $\mathrm{~N}$ & Database username of person who most recently updated record. \\
\hline Genus & varchar & 40 & 0 & $\mathrm{~N}$ & $\mathrm{~N}$ & Genus of taxon. \\
\hline Species & varchar & 40 & 0 & Y & $\mathrm{N}$ & Species of taxon. \\
\hline SubSpecies & varchar & 40 & 0 & $\mathrm{~N}$ & $\mathrm{~N}$ & Subspecies of taxon. \\
\hline TaxaClass & varchar & 50 & 0 & $\mathrm{~N}$ & $\mathrm{~N}$ & Class of taxon. \\
\hline TaxaFamily & varchar & 50 & 0 & $\mathrm{~N}$ & $\mathrm{~N}$ & Family of taxon. \\
\hline TaxaID & varchar & 25 & 0 & $\mathrm{Y}$ & $\mathrm{Y}$ & Taxonomic Serial Number used by ITIS.gov. See www.itis.gov. \\
\hline TaxaKingdom & varchar & 20 & 0 & $\mathrm{~N}$ & $\mathrm{~N}$ & Kingdom of taxon. \\
\hline TaxaOrder & varchar & 50 & 0 & $\mathrm{~N}$ & $\mathrm{~N}$ & Order of taxon. \\
\hline TaxaPhylum & varchar & 35 & 0 & $\mathrm{~N}$ & $\mathrm{~N}$ & Taxonomic phylum of the species. \\
\hline TaxaSubOrder & varchar & 35 & 0 & $\mathrm{~N}$ & $\mathrm{~N}$ & Taxonomic suborder of the species. \\
\hline
\end{tabular}

\section{TaxaObservationDetails Table Look Ups}

luCountMethod

\begin{tabular}{lcccccl}
\hline \multicolumn{1}{c}{ Field Name } & $\begin{array}{c}\text { Data } \\
\text { Type }\end{array}$ & $\begin{array}{c}\text { Field } \\
\text { Length }\end{array}$ & $\begin{array}{c}\text { Decimal } \\
\text { Places }\end{array}$ & $\begin{array}{c}\text { Required } \\
\text { Field }\end{array}$ & $\begin{array}{c}\text { Primary } \\
\text { Key }\end{array}$ & Definition \\
\hline CountMethod & varchar & 30 & 0 & $\mathrm{Y}$ & $\mathrm{N}$ & How the taxon was counted. \\
CountMethodID & int & 0 & 0 & $\mathrm{Y}$ & $\mathrm{Y}$ & CountMethod record identifier. \\
Description & varchar & 255 & 0 & $\mathrm{~N}$ & $\mathrm{~N}$ & Description of count method. \\
\hline
\end{tabular}

luDetectionMethod

\begin{tabular}{lcccccl}
\hline \multicolumn{1}{c}{ Field Name } & $\begin{array}{c}\text { Data } \\
\text { Type }\end{array}$ & $\begin{array}{c}\text { Field } \\
\text { Length }\end{array}$ & $\begin{array}{c}\text { Decimal } \\
\text { Places }\end{array}$ & $\begin{array}{c}\text { Required } \\
\text { Field }\end{array}$ & $\begin{array}{c}\text { Primary } \\
\text { Key }\end{array}$ & Definition \\
\hline DetectionMethod & varchar & 50 & 0 & $\mathrm{Y}$ & $\mathrm{N}$ & How the observed taxon was detected. \\
DetectionMethodCode & char & 3 & 0 & $\mathrm{Y}$ & $\mathrm{N}$ & Code for Detection Method. \\
DetectionMethodID & int & 0 & 0 & $\mathrm{Y}$ & $\mathrm{Y}$ & DetectionMethod record identifier. \\
\hline
\end{tabular}


luGender

\begin{tabular}{lcccccll}
\hline \multirow{2}{*}{ Field Name } & $\begin{array}{c}\text { Data } \\
\text { Type }\end{array}$ & $\begin{array}{c}\text { Field } \\
\text { Length }\end{array}$ & $\begin{array}{c}\text { Decimal } \\
\text { Places }\end{array}$ & $\begin{array}{c}\text { Required } \\
\text { Field }\end{array}$ & $\begin{array}{c}\text { Primary } \\
\text { Key }\end{array}$ & Definition \\
\hline Description & varchar & 255 & 0 & $\mathrm{~N}$ & $\mathrm{~N}$ & Description of gender. & \\
Gender & varchar & 30 & 0 & $\mathrm{Y}$ & $\mathrm{N}$ & Gender of taxon. \\
GenderID & int & 0 & 0 & $\mathrm{Y}$ & $\mathrm{Y}$ & Gender record identifier. & \\
\hline
\end{tabular}

luLifeStage

\begin{tabular}{lcccccll}
\hline Field Name & $\begin{array}{c}\text { Data } \\
\text { Type }\end{array}$ & $\begin{array}{c}\text { Field } \\
\text { Length }\end{array}$ & $\begin{array}{c}\text { Decimal } \\
\text { Places }\end{array}$ & $\begin{array}{c}\text { Required } \\
\text { Field }\end{array}$ & $\begin{array}{c}\text { Primary } \\
\text { Key }\end{array}$ & Definition \\
\hline Description & varchar & 350 & 0 & $\mathrm{~N}$ & $\mathrm{~N}$ & Description of life stage. & \\
LifeStage & varchar & 20 & 0 & $\mathrm{Y}$ & $\mathrm{N}$ & Life Stage of taxon. \\
LifeStageID & int & 0 & 0 & $\mathrm{Y}$ & $\mathrm{Y}$ & LifeStage record identifier. & \\
\hline
\end{tabular}

luTrapType

\begin{tabular}{ccccccc}
\hline $\begin{array}{c}\text { Field } \\
\text { Name }\end{array}$ & $\begin{array}{c}\text { Data } \\
\text { Type }\end{array}$ & $\begin{array}{c}\text { Field } \\
\text { Length }\end{array}$ & $\begin{array}{c}\text { Decimal } \\
\text { Places }\end{array}$ & $\begin{array}{c}\text { Required } \\
\text { Field }\end{array}$ & $\begin{array}{c}\text { Primary } \\
\text { Key }\end{array}$ & Definition \\
\hline TrapType & varchar & 35 & 0 & Y & Y & Type of trap used to capture the taxon. \\
\hline
\end{tabular}

luHabitatFeatureType

\begin{tabular}{lcccccc}
\hline \multicolumn{1}{c}{ Field Name } & $\begin{array}{c}\text { Data } \\
\text { Type }\end{array}$ & $\begin{array}{c}\text { Field } \\
\text { Length }\end{array}$ & $\begin{array}{c}\text { Decimal } \\
\text { Places }\end{array}$ & $\begin{array}{c}\text { Required } \\
\text { Field }\end{array}$ & $\begin{array}{c}\text { Primary } \\
\text { Key }\end{array}$ & Definition \\
\hline Description & varchar & 255 & 0 & $\mathrm{~N}$ & $\mathrm{~N}$ & Description of habitat feature type. \\
HabitatFeatureType & varchar & 30 & 0 & $\mathrm{Y}$ & $\mathrm{N}$ & Type of feature observed within habitat. \\
HabitatFeatureTypeID & int & 0 & 0 & $\mathrm{Y}$ & $\mathrm{Y}$ & HabitatFeatureType record identifier. \\
\hline
\end{tabular}

\section{TelemetryDetails Table Look Ups}

luLocationClass

\begin{tabular}{lcccccl}
\hline \multirow{2}{*}{ Field Name } & $\begin{array}{c}\text { Data } \\
\text { Type }\end{array}$ & $\begin{array}{c}\text { Field } \\
\text { Length }\end{array}$ & $\begin{array}{c}\text { Decimal } \\
\text { Places }\end{array}$ & $\begin{array}{c}\text { Required } \\
\text { Field }\end{array}$ & $\begin{array}{c}\text { Primary } \\
\text { Key }\end{array}$ & Definition \\
\hline Class & varchar & 2 & 0 & $\mathrm{Y}$ & $\mathrm{Y}$ & Location classification. \\
EstError & varchar & 60 & 0 & $\mathrm{~N}$ & $\mathrm{~N}$ & Estimated error of satellite location. \\
MsgsPerSatellitePass & varchar & 25 & 0 & $\mathrm{~N}$ & $\mathrm{~N}$ & Number of messages received per satellite pass. \\
Type & varchar & 10 & 0 & $\mathrm{~N}$ & $\mathrm{~N}$ & Type of location, Argos or GPS. \\
\hline
\end{tabular}

\section{TerrestrialHabitat Table Look Ups}

luPhenology

\begin{tabular}{ccccccl}
\hline $\begin{array}{c}\text { Field } \\
\text { Name }\end{array}$ & $\begin{array}{c}\text { Data } \\
\text { Type }\end{array}$ & $\begin{array}{c}\text { Field } \\
\text { Length }\end{array}$ & $\begin{array}{c}\text { Decimal } \\
\text { Places }\end{array}$ & $\begin{array}{c}\text { Required } \\
\text { Field }\end{array}$ & $\begin{array}{c}\text { Primary } \\
\text { Key }\end{array}$ & Definition \\
\hline Phenology & varchar & 10 & 0 & $\mathrm{Y}$ & $\mathrm{Y}$ & Seasonal timing of biological status. \\
\hline
\end{tabular}

luStructure

\begin{tabular}{cccccccc}
\hline $\begin{array}{c}\text { Field } \\
\text { Name }\end{array}$ & $\begin{array}{c}\text { Data } \\
\text { Type }\end{array}$ & $\begin{array}{c}\text { Field } \\
\text { Length }\end{array}$ & $\begin{array}{c}\text { Decimal } \\
\text { Places }\end{array}$ & $\begin{array}{c}\text { Required } \\
\text { Field }\end{array}$ & $\begin{array}{c}\text { Primary } \\
\text { Key }\end{array}$ & Definition \\
\hline Structure & varchar & 15 & 0 & Y & Y & Structure of feature at habitat. & \\
\hline
\end{tabular}


luTreeSize

\begin{tabular}{|c|c|c|c|c|c|c|}
\hline Field Name & $\begin{array}{l}\text { Data } \\
\text { Type }\end{array}$ & $\begin{array}{c}\text { Field } \\
\text { Length }\end{array}$ & $\begin{array}{c}\text { Decimal } \\
\text { Places }\end{array}$ & $\begin{array}{l}\text { Required } \\
\text { Field }\end{array}$ & $\begin{array}{c}\text { Primary } \\
\text { Key }\end{array}$ & Definition \\
\hline Description & varchar & 100 & 0 & $\mathrm{~N}$ & $\mathrm{~N}$ & Description of the range of measurements. \\
\hline TreeSizeID & int & 0 & 0 & Y & $\mathrm{Y}$ & TreeSize record identifier. \\
\hline
\end{tabular}

luVegetationStandSize

\begin{tabular}{lcccccc}
\hline \multicolumn{1}{c}{ Field Name } & $\begin{array}{c}\text { Data } \\
\text { Type }\end{array}$ & $\begin{array}{c}\text { Field } \\
\text { Length }\end{array}$ & $\begin{array}{c}\text { Decimal } \\
\text { Places }\end{array}$ & $\begin{array}{c}\text { Required } \\
\text { Field }\end{array}$ & $\begin{array}{c}\text { Primary } \\
\text { Key }\end{array}$ & Definition \\
\hline VegetationStandSize & varchar & 50 & 0 & $\mathrm{Y}$ & $\mathrm{N}$ & Size of vegetation stands present in habitat. \\
$\begin{array}{l}\text { VegetationStandSize } \\
\begin{array}{l}\text { Code } \\
\text { VegetationStandSizeID }\end{array}\end{array}$ & varchar & 2 & 0 & $\mathrm{~N}$ & $\mathrm{~N}$ & Code for vegetation stand size. \\
\hline
\end{tabular}

\section{WaterPhysicalObservation Table Look Ups}

Hardness

\begin{tabular}{lccccccl}
\hline Field Name & $\begin{array}{c}\text { Data } \\
\text { Type }\end{array}$ & $\begin{array}{c}\text { Field } \\
\text { Length }\end{array}$ & $\begin{array}{c}\text { Decimal } \\
\text { Places }\end{array}$ & $\begin{array}{c}\text { Required } \\
\text { Field }\end{array}$ & $\begin{array}{c}\text { Primary } \\
\text { Key }\end{array}$ & Definition \\
\hline Hardness & varchar & 30 & 0 & $\mathrm{Y}$ & $\mathrm{N}$ & Categorical levels of water hardness in mg/L. \\
HardnessID & char & 1 & 0 & $\mathrm{Y}$ & $\mathrm{Y}$ & Hardness record identifier. & \\
\hline
\end{tabular}

Transparency

\begin{tabular}{lcccccl}
\hline Field Name & $\begin{array}{c}\text { Data } \\
\text { Type }\end{array}$ & $\begin{array}{c}\text { Field } \\
\text { Length }\end{array}$ & $\begin{array}{c}\text { Decimal } \\
\text { Places }\end{array}$ & $\begin{array}{c}\text { Required } \\
\text { Field }\end{array}$ & $\begin{array}{c}\text { Primary } \\
\text { Key }\end{array}$ & Definition \\
\hline Transparency & varchar & 20 & 0 & $\mathrm{Y}$ & $\mathrm{N}$ & Categorical description of how clear or silted the water is. \\
TranspID & char & 1 & 0 & $\mathrm{Y}$ & $\mathrm{Y}$ & Abbreviation/code for transparency of water. \\
\hline
\end{tabular}

TranspMeth

\begin{tabular}{lccccccl}
\hline Field Name & $\begin{array}{c}\text { Data } \\
\text { Type }\end{array}$ & $\begin{array}{c}\text { Field } \\
\text { Length }\end{array}$ & $\begin{array}{c}\text { Decimal } \\
\text { Places }\end{array}$ & $\begin{array}{c}\text { Required } \\
\text { Field }\end{array}$ & $\begin{array}{c}\text { Primary } \\
\text { Key }\end{array}$ & Definition \\
\hline TranspMeth & varchar & 20 & 0 & $\mathrm{Y}$ & $\mathrm{N}$ & Quantitative transparency method used. \\
TranspMID & char & 1 & 0 & $\mathrm{Y}$ & $\mathrm{Y}$ & TranspMeth record identifier. \\
\hline
\end{tabular}

TurbUnits

\begin{tabular}{cccccccl}
\hline $\begin{array}{c}\text { Field } \\
\text { Name }\end{array}$ & $\begin{array}{c}\text { Data } \\
\text { Type }\end{array}$ & $\begin{array}{c}\text { Field } \\
\text { Length }\end{array}$ & $\begin{array}{c}\text { Decimal } \\
\text { Places }\end{array}$ & $\begin{array}{c}\text { Required } \\
\text { Field }\end{array}$ & $\begin{array}{c}\text { Primary } \\
\text { Key }\end{array}$ & Definition \\
\hline TurbUID & char & 1 & 0 & $\mathrm{Y}$ & $\mathrm{Y}$ & TurbUnits record identifier. & \\
TurbUnits & varchar & 20 & 0 & $\mathrm{Y}$ & $\mathrm{N}$ & Turbidimeter units used for Turbidity measurement. \\
\hline
\end{tabular}


WaterColor

\begin{tabular}{lcccccc}
\hline Field Name & $\begin{array}{c}\text { Data } \\
\text { Type }\end{array}$ & $\begin{array}{c}\text { Field } \\
\text { Length }\end{array}$ & $\begin{array}{c}\text { Decimal } \\
\text { Places }\end{array}$ & $\begin{array}{c}\text { Required } \\
\text { Field }\end{array}$ & $\begin{array}{c}\text { Primary } \\
\text { Key }\end{array}$ & Definition \\
\hline WaterColor & varchar & 10 & 0 & Y & Y & The color of the water as observed from above \\
\hline
\end{tabular}

WeatherObservation Table Look Ups

luBarometerTrend

\begin{tabular}{|c|c|c|c|c|c|c|}
\hline Field Name & $\begin{array}{l}\text { Data } \\
\text { Type }\end{array}$ & $\begin{array}{c}\text { Field } \\
\text { Length }\end{array}$ & $\begin{array}{c}\text { Decimal } \\
\text { Places }\end{array}$ & $\begin{array}{l}\text { Required } \\
\text { Field }\end{array}$ & $\begin{array}{c}\text { Primary } \\
\text { Key }\end{array}$ & Definition \\
\hline BarometerTrend & varchar & 10 & 0 & $\mathrm{Y}$ & $\mathrm{Y}$ & Direction in which barometric readings are going. \\
\hline \multicolumn{7}{|l|}{ luDirection } \\
\hline Field Name & $\begin{array}{l}\text { Data } \\
\text { Type }\end{array}$ & $\begin{array}{l}\text { Field } \\
\text { Length }\end{array}$ & $\begin{array}{l}\text { Decimal } \\
\text { Places }\end{array}$ & $\begin{array}{l}\text { Required } \\
\text { Field }\end{array}$ & $\begin{array}{c}\text { Primary } \\
\text { Key }\end{array}$ & Definition \\
\hline DirectionCode & varchar & 3 & 0 & $\mathrm{Y}$ & $\mathrm{Y}$ & Abbreviation for cardinal direction. \\
\hline
\end{tabular}

luMoonPhase

\begin{tabular}{lcccccl}
\hline \multicolumn{1}{c}{ Field Name } & $\begin{array}{c}\text { Data } \\
\text { Type }\end{array}$ & $\begin{array}{c}\text { Field } \\
\text { Length }\end{array}$ & $\begin{array}{c}\text { Decimal } \\
\text { Places }\end{array}$ & $\begin{array}{c}\text { Required } \\
\text { Field }\end{array}$ & $\begin{array}{c}\text { Primary } \\
\text { Key }\end{array}$ & Definition \\
\hline MoonPhase & varchar & 20 & 0 & $\mathrm{Y}$ & $\mathrm{N}$ & Phase of the lunar cycle. \\
MoonPhaseCode & varchar & 2 & 0 & $\mathrm{Y}$ & $\mathrm{Y}$ & Abbreviation/code for moon phase. \\
\hline
\end{tabular}

luObsTiming

\begin{tabular}{ccccccc}
\hline $\begin{array}{c}\text { Field } \\
\text { Name }\end{array}$ & $\begin{array}{c}\text { Data } \\
\text { Type }\end{array}$ & $\begin{array}{c}\text { Field } \\
\text { Length }\end{array}$ & $\begin{array}{c}\text { Decimal } \\
\text { Places }\end{array}$ & $\begin{array}{c}\text { Required } \\
\text { Field }\end{array}$ & $\begin{array}{c}\text { Primary } \\
\text { Key }\end{array}$ & Definition \\
\hline ObsTiming & varchar & 10 & 0 & $\mathrm{~N}$ & $\mathrm{Y}$ & $\begin{array}{c}\text { Timing of the observation relative to the survey, or where on a scale the } \\
\text { observation occurred. }\end{array}$ \\
\hline
\end{tabular}

luSkyObservation

\begin{tabular}{lcccccl}
\hline \multicolumn{1}{c}{ Field Name } & $\begin{array}{c}\text { Data } \\
\text { Type }\end{array}$ & $\begin{array}{c}\text { Field } \\
\text { Length }\end{array}$ & $\begin{array}{c}\text { Decimal } \\
\text { Places }\end{array}$ & $\begin{array}{c}\text { Required } \\
\text { Field }\end{array}$ & $\begin{array}{c}\text { Primary } \\
\text { Key }\end{array}$ & Definition \\
\hline Description & varchar & 100 & 0 & $\mathrm{~N}$ & $\mathrm{~N}$ & Description of the sky condition. \\
SkyObservation & varchar & 30 & 0 & $\mathrm{Y}$ & $\mathrm{N}$ & Sky conditions. \\
SkyObservationID & int & 0 & 0 & $\mathrm{Y}$ & $\mathrm{Y}$ & SkyObservation record identifier. \\
\hline
\end{tabular}

luWindBeaufortScale

\begin{tabular}{lcccccl}
\hline \multicolumn{1}{c}{ Field Name } & $\begin{array}{c}\text { Data } \\
\text { Type }\end{array}$ & $\begin{array}{c}\text { Field } \\
\text { Length }\end{array}$ & $\begin{array}{c}\text { Decimal } \\
\text { Places }\end{array}$ & $\begin{array}{c}\text { Required } \\
\text { Field }\end{array}$ & $\begin{array}{c}\text { Primary } \\
\text { Key }\end{array}$ & Definition \\
\hline Description & varchar & 20 & 0 & $\mathrm{Y}$ & $\mathrm{N}$ & Description of Beaufort Scale condition. \\
Indicators & varchar & 100 & 0 & $\mathrm{~N}$ & $\mathrm{~N}$ & Indicators used to determine Beaufort Scale level. \\
MinMPH & int & 0 & 0 & $\mathrm{Y}$ & $\mathrm{N}$ & Minimum wind speed, in miles per hour. \\
MPHRange & varchar & 10 & 0 & $\mathrm{~N}$ & $\mathrm{~N}$ & Range of wind speeds, in miles per hour. \\
WindBeaufortScaleID & int & 0 & 0 & $\mathrm{Y}$ & $\mathrm{Y}$ & WindBeaufortScale record identifier. \\
\hline
\end{tabular}


SECTION III: LOOK UP DOMAIN

TABLES

\section{ConstrainBy}

\begin{tabular}{ll}
\hline ConstrainedBy & \multicolumn{1}{c}{ ConstraintDefinition } \\
\hline TIME & Time constrained survey \\
AREA & Area constrained survey \\
DIST & Distance constrained survey \\
\hline
\end{tabular}

\section{Hardness}

\begin{tabular}{cl}
\hline HardnessID & \multicolumn{1}{c}{ Hardness } \\
\hline 1 & Soft $(0 \sim 60 \mathrm{mg} / \mathrm{L})$ \\
2 & Moderately hard $(61 \sim 120 \mathrm{mg} / \mathrm{L})$ \\
3 & Hard $(121 \sim 180 \mathrm{mg} / \mathrm{L})$ \\
4 & Very hard (over $180 \mathrm{mg} / \mathrm{L})$ \\
\hline
\end{tabular}

\section{IDType}

\begin{tabular}{|c|c|}
\hline IDType & Description \\
\hline 01 & Pattern recognition \\
\hline 02 & PIT tag \\
\hline 03 & Scale clip \\
\hline 04 & Tail clip \\
\hline 05 & Toe clip \\
\hline 06 & VIE \\
\hline 09 & Other \\
\hline 10 & Name \\
\hline 11 & Radio transmitter \\
\hline 12 & GPS collar \\
\hline 13 & Bead pattern \\
\hline 14 & Ear tag \\
\hline 15 & Ankle band \\
\hline 16 & Wing marker \\
\hline 17 & Hair clip \\
\hline 18 & PIT tag - Avid \\
\hline 19 & PIT tag - other \\
\hline 20 & Notched-plastron \\
\hline 21 & Notched-carapace \\
\hline 22 & Notched-plastron/carapace \\
\hline 23 & Scute tag \\
\hline
\end{tabular}

\section{luAbundanceCategory}

\begin{tabular}{cll}
\hline AbundanceCategoryCode & AbundanceCategory & \multicolumn{1}{c}{ Description } \\
\hline A & Abundant & Specimens abundantly present \\
C & Common & Specimens continuously in view without actively searching \\
CS & Large contiguous stands & Relatively many plants at site \\
FP & Few plants & Relatively few plants at site \\
N & Not present & No specimens observed \\
NA & Not applicable & Does not apply \\
Sc & Scarce & Very few specimens observed, individual specimens are hard to find when searching \\
So & Solitary & Only one individual specimen observed \\
SP & Scattered small patches & Relatively medium number of plants at site \\
U & Uncommon & Scattered specimens observed, individual specimens are observed without actively \\
& & searching for them \\
UK & Unknown & Unknown value \\
\hline
\end{tabular}




\section{luActivityType}

\begin{tabular}{cll}
\hline ActivityTypeID & \multicolumn{1}{c}{ ActivityType } & Description \\
\hline 1 & Fence installation & \\
2 & Fence removal & \\
3 & Trail installation & \\
4 & Trail removal & \\
5 & Facility installation \\
6 & Facility removal \\
7 & Fuel reduction \\
8 & Erosion control \\
99 & Other \\
\hline
\end{tabular}

\section{luAffiliateRole}

\begin{tabular}{|c|c|}
\hline Role & Description \\
\hline \multicolumn{2}{|l|}{ Applicant } \\
\hline ARMI organizational unit & $\begin{array}{l}\text { ARMI program region/office/ } \\
\text { organization }\end{array}$ \\
\hline \multicolumn{2}{|l|}{ Caller } \\
\hline \multicolumn{2}{|l|}{ Collector } \\
\hline \multicolumn{2}{|l|}{ Contact } \\
\hline \multicolumn{2}{|l|}{ Contractor } \\
\hline Data reviewer, in field & $\begin{array}{l}\text { Reviews raw survey data while still } \\
\text { on site }\end{array}$ \\
\hline Data steward & $\begin{array}{l}\text { Contact person for questions about the } \\
\text { data }\end{array}$ \\
\hline \multicolumn{2}{|l|}{ Field coordinator } \\
\hline Investigator & Principal Investigator \\
\hline \multicolumn{2}{|l|}{ Observer } \\
\hline \multicolumn{2}{|l|}{ Observer/Recorder } \\
\hline \multicolumn{2}{|l|}{ On-site contact } \\
\hline \multicolumn{2}{|l|}{ Operator } \\
\hline \multicolumn{2}{|l|}{ Owner } \\
\hline \multicolumn{2}{|l|}{ Partner } \\
\hline \multicolumn{2}{|l|}{ Processor } \\
\hline Recorder & $\begin{array}{l}\text { Recorder of data, either paper or } \\
\text { electronic }\end{array}$ \\
\hline \multicolumn{2}{|l|}{ Study lead } \\
\hline \multicolumn{2}{|l|}{ Submitter } \\
\hline Volunteer & \\
\hline
\end{tabular}

\begin{tabular}{clc}
\hline AffiliateTypeID & \multicolumn{1}{c}{ AffiliateType } & Description \\
\hline-1 & Undefined organization & NA \\
0 & Unknown & NA \\
1 & Individual & NA
\end{tabular}

\begin{tabular}{clc}
\multicolumn{2}{l}{ IuAffiliateType-Continued } \\
\hline AffiliateTypeID & \multicolumn{1}{c}{ AffiliateType } & Description \\
\hline 2 & State government organization & NA \\
3 & Federal government organization & NA \\
4 & Local government organization & NA \\
5 & Private organization & NA \\
6 & Tribal organization & NA \\
7 & Non-profit organization & NA \\
8 & Educational organization & NA \\
9 & Council, board or association & NA \\
10 & Museum & NA \\
\hline
\end{tabular}

\section{luAgeMetric}

\begin{tabular}{ll}
\hline & AgeMetric \\
\hline Days & \\
Months & \\
Years & \\
\hline
\end{tabular}

\section{luAgePelage}

\begin{tabular}{cll}
\hline AgePelageCode & & Description \\
\hline A & Adult & \\
J & Juvenile & \\
L & Larval & \\
NA & NA & \\
SA & Subadult & \\
\hline
\end{tabular}

\section{luAmtSurveyedUnit}

\begin{tabular}{ll}
\hline & AmountSurveyed Unit \\
\hline Percent & \\
\hline
\end{tabular}

\section{IuBarometerTrend}

\begin{tabular}{ll}
\hline & BarometerTrend \\
\hline Falling & \\
Rising & \\
Stable & \\
Unknown & \\
\hline
\end{tabular}

\section{luBaskingAreas}

\begin{tabular}{ll}
\hline & BaskingArea \\
\hline Bedrock & \\
Boulders & \\
Emergent Vegetation &
\end{tabular}


luBaskingAreas-Continued

\begin{tabular}{l}
\hline \multicolumn{1}{c}{ BaskingArea } \\
\hline Fallen logs \\
Open banks \\
Other \\
Sandy banks \\
Sunny rocks \\
Woody debris \\
\hline
\end{tabular}

\section{luBaskingAreasContext}

\begin{tabular}{ll}
\hline & Context \\
\hline Aquatic & \\
Arboreal & \\
Terrestrial & \\
\hline
\end{tabular}

\section{IuBehavior}

Behavior

Active (on surface)

Basking

Behavior undefined (Sea Otter)

Bicycling

Broadcast calling

Co-occuring

Copulation

Distraction display

Disturbance/Release calling

Driving ATV/Motorcycle

Driving vehicle

Flushed

Food carry

Foraging

Head butting

Host

Material carry
luBehavior-Continued

\begin{tabular}{l}
\hline \multicolumn{1}{c}{ Behavior } \\
\hline Nectaring \\
Other \\
Parasitising \\
Resting \\
Roosting \\
Typical travel \\
Unknown \\
USGS (or Wildlife) surveys \\
\hline
\end{tabular}

\section{luCollectionType}

\begin{tabular}{ll}
\hline & CollectionType \\
\hline Filter & \\
Hair & \\
Live animal \\
Other \\
Scat \\
Shed \\
Swab \\
Tissue \\
Unknown \\
Voucher
\end{tabular}

\section{luCollectionUses}

\section{CollectionUse}

Diet composition analysis

Genetics

Histology

ID confirmation

Identification

Repository donation

Unknown

\section{luCountMethod}

\begin{tabular}{cll}
\hline CountMethodID & \multicolumn{1}{c}{ CountMethod } & \multicolumn{1}{c}{ Description } \\
\hline 1 & Actual count & Total actual count of individuals \\
2 & Estimated count & Estimated total count of individuals \\
3 & NAAMP calling code 0 & Not heard calling \\
4 & NAAMP calling code 1 & Individuals clearly heard \\
5 & NAAMP calling code 2 & Chorusing with discernible individuals \\
6 & NAAMP calling code 3 & Chorusing, no individuals discernible \\
7 & Estimated count (egg masses) & Estimated total count of egg masses
\end{tabular}


IuCountMethod-Continued

\begin{tabular}{cll}
\hline CountMethodID & \multicolumn{1}{c}{ CountMethod } & \multicolumn{1}{c}{ Description } \\
\hline 8 & Actual count (egg masses) & Actual count of individual egg masses \\
9 & Estimated percentage & Estimated percent composition \\
98 & Not applicable & Not applicable to particular observation or study \\
99 & Unknown & Unknown or data not recorded. \\
\hline
\end{tabular}

\section{luCountry}

\begin{tabular}{|c|c|c|}
\hline $\begin{array}{c}\text { Country } \\
\text { Code }\end{array}$ & Country & $\begin{array}{l}\text { Country } \\
\text { CodeA3 }\end{array}$ \\
\hline $\mathrm{AD}$ & Andorra & AND \\
\hline $\mathrm{AE}$ & United Arab Emirates & ARE \\
\hline $\mathrm{AF}$ & Afghanistan & AFG \\
\hline $\mathrm{AG}$ & Antigua and Barbuda & ATG \\
\hline AI & Anguilla & AIA \\
\hline $\mathrm{AL}$ & Albania & ALB \\
\hline $\mathrm{AM}$ & Armenia & ARM \\
\hline $\mathrm{AN}$ & Netherlands Antilles & ANT \\
\hline $\mathrm{AO}$ & Angola & AGO \\
\hline AQ & Antarctica & ATA \\
\hline $\mathrm{AR}$ & Argentina & ARG \\
\hline AS & American Samoa & ASM \\
\hline AT & Austria & AUT \\
\hline $\mathrm{AU}$ & Australia & AUS \\
\hline AW & Aruba & $\mathrm{ABW}$ \\
\hline $\mathrm{AX}$ & Åland & ALA \\
\hline $\mathrm{AZ}$ & Azerbaijan & AZE \\
\hline BA & Bosnia and Herzegovina & $\mathrm{BIH}$ \\
\hline $\mathrm{BB}$ & Barbados & $\mathrm{BRB}$ \\
\hline $\mathrm{BD}$ & Bangladesh & BGD \\
\hline $\mathrm{BE}$ & Belgium & BEL \\
\hline $\mathrm{BF}$ & Burkina Faso & BFA \\
\hline BG & Bulgaria & BGR \\
\hline $\mathrm{BH}$ & Bahrain & BHR \\
\hline $\mathrm{BI}$ & Burundi & BDI \\
\hline $\mathrm{BJ}$ & Benin & BEN \\
\hline $\mathrm{BM}$ & Bermuda & BMU \\
\hline $\mathrm{BN}$ & Brunei Darussalam & BRN \\
\hline $\mathrm{BO}$ & Bolivia & BOL \\
\hline $\mathrm{BR}$ & Brazil & BRA \\
\hline BS & Bahamas & BHS \\
\hline BT & Bhutan & BTN \\
\hline BV & Bouvet Island & BVT \\
\hline BW & Botswana & BWA \\
\hline BY & Belarus & BLR \\
\hline $\mathrm{BZ}$ & Belize & BLZ \\
\hline
\end{tabular}

luCountry-Continued

\begin{tabular}{ccc}
\hline $\begin{array}{c}\text { Country } \\
\text { Code }\end{array}$ & Country & $\begin{array}{c}\text { Country } \\
\text { CodeA3 }\end{array}$ \\
\hline CA & Canada & CAN
\end{tabular}

CC Cocos (Keeling) Islands CCK

CD Congo (Kinshasa) COD

CF Central African Republic CAF

CG Congo (Brazzaville) COG

$\mathrm{CH}$ Switzerland $\mathrm{CHE}$

CI Côte d'Ivoire CIV

CK Cook Islands COK

CL Chile CHL

CM Cameroon CMR

CN China CHN

$\mathrm{CO}$ Colombia COL

CR Costa Rica CRI

CS Serbia and Montenegro SCG

CU Cuba CUB

CV Cape Verde CPV

CX Christmas Island CXR

CY Cyprus CYP

CZ Czech Republic CZE

DE Germany DEU

DJ Djibouti DJI

DK Denmark DNK

DM Dominica DMA

DO Dominican Republic DOM

DZ Algeria DZA

EC Ecuador ECU

EE Estonia EST

EG Egypt EGY

EH Western Sahara ESH

ER Eritrea ERI

ES Spain ESP

ET Ethiopia ETH

FI Finland FIN

FJ Fiji FJI

FK Falkland Islands $\quad$ FLK

FM Micronesia FSM 


\begin{tabular}{|c|c|c|c|c|c|}
\hline \multicolumn{3}{|c|}{ luCountry-Continued } & \multicolumn{3}{|c|}{ luCountry-Continued } \\
\hline $\begin{array}{c}\text { Country } \\
\text { Code }\end{array}$ & Country & $\begin{array}{l}\text { Country } \\
\text { CodeA3 }\end{array}$ & $\begin{array}{c}\text { Country } \\
\text { Code }\end{array}$ & Country & $\begin{array}{l}\text { Country } \\
\text { CodeA3 }\end{array}$ \\
\hline FO & Faroe Islands & FRO & KM & Comoros & $\mathrm{COM}$ \\
\hline FR & France & FRA & $\mathrm{KN}$ & Saint Kitts and Nevis & KNA \\
\hline GA & Gabon & GAB & KP & Korea, North & PRK \\
\hline GB & United Kingdom & GBR & $\mathrm{KR}$ & Korea, South & KOR \\
\hline GD & Grenada & GRD & KW & Kuwait & KWT \\
\hline GE & Georgia & GEO & KY & Cayman Islands & CYM \\
\hline GF & French Guiana & GUF & $\mathrm{KZ}$ & Kazakhstan & KAZ \\
\hline $\mathrm{GH}$ & Ghana & GHA & LA & Laos & LAO \\
\hline GI & Gibraltar & GIB & LB & Lebanon & LBN \\
\hline GL & Greenland & GRL & $\mathrm{LC}$ & Saint Lucia & LCA \\
\hline GM & Gambia & GMB & LI & Liechtenstein & LIE \\
\hline GN & Guinea & GIN & LK & Sri Lanka & LKA \\
\hline GP & Guadeloupe & GLP & LR & Liberia & LBR \\
\hline GQ & Equatorial Guinea & GNQ & LS & Lesotho & LSO \\
\hline GR & Greece & GRC & LT & Lithuania & LTU \\
\hline GS & South Georgia and South Sandwich Islands & SGS & $\mathrm{LU}$ & Luxembourg & LUX \\
\hline GT & Guatemala & GTM & LV & Latvia & LVA \\
\hline GU & Guam & GUM & LY & Libya & LBY \\
\hline GW & Guinea-Bissau & GNB & MA & Morocco & MAR \\
\hline GY & Guyana & GUY & $\mathrm{MC}$ & Monaco & $\mathrm{MCO}$ \\
\hline HK & Hong Kong & HKG & MD & Moldova & MDA \\
\hline HM & Heard and McDonald Islands & HMD & MG & Madagascar & MDG \\
\hline $\mathrm{HN}$ & Honduras & HND & MH & Marshall Islands & MHL \\
\hline HR & Croatia & HRV & MK & Macedonia & MKD \\
\hline HT & Haiti & HTI & ML & Mali & MLI \\
\hline $\mathrm{HU}$ & Hungary & HUN & MM & Myanmar & MMR \\
\hline ID & Indonesia & IDN & $\mathrm{MN}$ & Mongolia & MNG \\
\hline IE & Ireland & IRL & MO & Macau & MAC \\
\hline IL & Israel & ISR & MP & Northern Mariana Islands & MNP \\
\hline IN & India & IND & MQ & Martinique & MTQ \\
\hline IO & British Indian Ocean Territory & IOT & MR & Mauritania & MRT \\
\hline IQ & Iraq & IRQ & MS & Montserrat & MSR \\
\hline IR & Iran & IRN & MT & Malta & MLT \\
\hline IS & Iceland & ISL & MU & Mauritius & MUS \\
\hline IT & Italy & ITA & MV & Maldives & MDV \\
\hline $\mathrm{JM}$ & Jamaica & JAM & MW & Malawi & MWI \\
\hline $\mathrm{JO}$ & Jordan & JOR & MX & Mexico & MEX \\
\hline JP & Japan & JPN & MY & Malaysia & MYS \\
\hline $\mathrm{KE}$ & Kenya & KEN & $\mathrm{MZ}$ & Mozambique & $\mathrm{MOZ}$ \\
\hline KG & Kyrgyzstan & KGZ & NA & Namibia & NAM \\
\hline $\mathrm{KH}$ & Cambodia & KHM & $\mathrm{NC}$ & New Caledonia & NCL \\
\hline KI & Kiribati & KIR & $\mathrm{NE}$ & Niger & NER \\
\hline
\end{tabular}


luCountry-Continued

\begin{tabular}{|c|c|c|}
\hline $\begin{array}{c}\text { Country } \\
\text { Code }\end{array}$ & Country & $\begin{array}{l}\text { Country } \\
\text { CodeA3 }\end{array}$ \\
\hline $\mathrm{NF}$ & Norfolk Island & NFK \\
\hline NG & Nigeria & NGA \\
\hline NI & Nicaragua & NIC \\
\hline NL & Netherlands & NLD \\
\hline NO & Norway & NOR \\
\hline NP & Nepal & NPL \\
\hline NR & Nauru & NRU \\
\hline NU & Niue & NIU \\
\hline $\mathrm{NZ}$ & New Zealand & NZL \\
\hline $\mathrm{OM}$ & Oman & OMN \\
\hline PA & Panama & PAN \\
\hline PE & Peru & PER \\
\hline $\mathrm{PF}$ & French Polynesia & PYF \\
\hline PG & Papua New Guinea & PNG \\
\hline PH & Philippines & PHL \\
\hline PL & Poland & POL \\
\hline PM & Saint Pierre and Miquelon & SPM \\
\hline $\mathrm{PN}$ & Pitcairn & PCN \\
\hline PR & Puerto Rico & PRI \\
\hline $\begin{array}{c}\text { Primary } \\
\text { Key }\end{array}$ & Pakistan & PAK \\
\hline PS & Palestine & PSE \\
\hline PT & Portugal & PRT \\
\hline PW & Palau & PLW \\
\hline PY & Paraguay & PRY \\
\hline QA & Qatar & QAT \\
\hline $\mathrm{RE}$ & Reunion & REU \\
\hline RO & Romania & ROU \\
\hline RU & Russian Federation & RUS \\
\hline RW & Rwanda & RWA \\
\hline SA & Saudi Arabia & SAU \\
\hline SB & Solomon Islands & SLB \\
\hline $\mathrm{SC}$ & Seychelles & SYC \\
\hline $\mathrm{SD}$ & Sudan & SDN \\
\hline $\mathrm{SE}$ & Sweden & SWE \\
\hline SG & Singapore & SGP \\
\hline $\mathrm{SH}$ & Saint Helena & SHN \\
\hline SI & Slovenia & SVN \\
\hline $\mathrm{SJ}$ & Svalbard and Jan Mayen Islands & SJM \\
\hline SK & Slovakia & SVK \\
\hline SL & Sierra Leone & SLE \\
\hline SM & San Marino & SMR \\
\hline SN & Senegal & SEN \\
\hline
\end{tabular}

luCountry-Continued

\begin{tabular}{ccc}
\hline $\begin{array}{c}\text { Country } \\
\text { Code }\end{array}$ & Country & $\begin{array}{c}\text { Country } \\
\text { CodeA3 }\end{array}$ \\
\hline SO & Somalia & SOM
\end{tabular}

SR Suriname

SUR

ST Sao Tome and Principe STP

SV El Salvador SLV

SY Syria SYR

SZ Swaziland SWZ

TC Turks and Caicos Islands TCA

TD Chad TCD

TF French Southern Lands $\quad$ ATF

TG Togo TGO

TH Thailand THA

TJ Tajikistan TJK

TK Tokelau TKL

TL Timor-Leste TLS

TM Turkmenistan TKM

TN Tunisia TUN

TO Tonga TON

TR Turkey TUR

TT Trinidad and Tobago TTO

TV Tuvalu TUV

TW Taiwan TWN

TZ Tanzania TZA

UA Ukraine UKR

UG Uganda UGA

UM United States Minor Outlying Islands UMI

US United States of America USA

UY Uruguay URY

UZ Uzbekistan UZB

VA Vatican City VAT

VC Saint Vincent and the Grenadines VCT

VE Venezuela VEN

VG Virgin Islands, British VGB

VI Virgin Islands, U.S. VIR

VN Viet Nam VNM

VU Vanuatu VUT

WF Wallis and Futuna Islands WLF

WS Samoa WSM

YE Yemen YEM

YT Mayotte MYT

ZA South Africa ZAF

ZM Zambia ZMB

ZW Zimbabwe ZWE 
luCounty

\begin{tabular}{|c|c|c|c|}
\hline $\begin{array}{l}\text { County } \\
\text { ID }\end{array}$ & County & $\begin{array}{l}\text { County } \\
\text { FIPSCode }\end{array}$ & $\begin{array}{l}\text { State } \\
\text { Code }\end{array}$ \\
\hline 1 & Alameda & 1 & $\mathrm{CA}$ \\
\hline 2 & Alpine & 3 & $\mathrm{CA}$ \\
\hline 3 & Amador & 5 & $\mathrm{CA}$ \\
\hline 4 & Butte & 7 & $\mathrm{CA}$ \\
\hline 5 & Calaveras & 9 & $\mathrm{CA}$ \\
\hline 6 & Colusa & 11 & $\mathrm{CA}$ \\
\hline 7 & Contra Costa & 13 & $\mathrm{CA}$ \\
\hline 8 & Del Norte & 15 & $\mathrm{CA}$ \\
\hline 9 & El Dorado & 17 & $\mathrm{CA}$ \\
\hline 10 & Fresno & 19 & $\mathrm{CA}$ \\
\hline 11 & Glenn & 21 & $\mathrm{CA}$ \\
\hline 12 & Humboldt & 23 & $\mathrm{CA}$ \\
\hline 13 & Imperial & 25 & $\mathrm{CA}$ \\
\hline 14 & Inyo & 27 & $\mathrm{CA}$ \\
\hline 15 & Kern & 29 & $\mathrm{CA}$ \\
\hline 16 & Kings & 31 & $\mathrm{CA}$ \\
\hline 17 & Lake & 33 & $\mathrm{CA}$ \\
\hline 18 & Lassen & 35 & $\mathrm{CA}$ \\
\hline 19 & Los Angeles & 37 & $\mathrm{CA}$ \\
\hline 20 & Madera & 39 & $\mathrm{CA}$ \\
\hline 21 & Marin & 41 & $\mathrm{CA}$ \\
\hline 22 & Mariposa & 43 & $\mathrm{CA}$ \\
\hline 23 & Mendocino & 45 & $\mathrm{CA}$ \\
\hline 24 & Merced & 47 & $\mathrm{CA}$ \\
\hline 25 & Modoc & 49 & $\mathrm{CA}$ \\
\hline 26 & Mono & 51 & $\mathrm{CA}$ \\
\hline 27 & Monterey & 53 & $\mathrm{CA}$ \\
\hline 28 & Napa & 55 & CA \\
\hline 29 & Nevada & 57 & $\mathrm{CA}$ \\
\hline 30 & Orange & 59 & $\mathrm{CA}$ \\
\hline 31 & Placer & 61 & $\mathrm{CA}$ \\
\hline 32 & Plumas & 63 & $\mathrm{CA}$ \\
\hline 33 & Riverside & 65 & CA \\
\hline 34 & Sacramento & 67 & $\mathrm{CA}$ \\
\hline 35 & San Benito & 69 & $\mathrm{CA}$ \\
\hline 36 & San Bernardino & 71 & $\mathrm{CA}$ \\
\hline 37 & San Diego & 73 & $\mathrm{CA}$ \\
\hline 38 & San Francisco & 75 & $\mathrm{CA}$ \\
\hline 39 & San Joaquin & 77 & $\mathrm{CA}$ \\
\hline 40 & San Luis Obispo & 79 & $\mathrm{CA}$ \\
\hline 41 & San Mateo & 81 & $\mathrm{CA}$ \\
\hline 42 & Santa Barbara & 83 & $\mathrm{CA}$ \\
\hline 43 & Santa Clara & 85 & $\mathrm{CA}$ \\
\hline 44 & Santa Cruz & 87 & $\mathrm{CA}$ \\
\hline
\end{tabular}

\section{luCounty-Continued}

\begin{tabular}{|c|c|c|c|}
\hline $\begin{array}{l}\text { County } \\
\text { ID }\end{array}$ & County & $\begin{array}{l}\text { County } \\
\text { FIPSCode }\end{array}$ & $\begin{array}{l}\text { State } \\
\text { Code }\end{array}$ \\
\hline 45 & Shasta & 89 & $\mathrm{CA}$ \\
\hline 46 & Sierra & 91 & $\mathrm{CA}$ \\
\hline 47 & Siskiyou & 93 & $\mathrm{CA}$ \\
\hline 48 & Solano & 95 & $\mathrm{CA}$ \\
\hline 49 & Sonoma & 97 & CA \\
\hline 50 & Stanislaus & 99 & $\mathrm{CA}$ \\
\hline 51 & Sutter & 101 & $\mathrm{CA}$ \\
\hline 52 & Tehama & 103 & $\mathrm{CA}$ \\
\hline 53 & Trinity & 105 & $\mathrm{CA}$ \\
\hline 54 & Tulare & 107 & $\mathrm{CA}$ \\
\hline 55 & Tuolumne & 109 & $\mathrm{CA}$ \\
\hline 56 & Ventura & 111 & $\mathrm{CA}$ \\
\hline 57 & Yolo & 113 & $\mathrm{CA}$ \\
\hline 58 & Yuba & 115 & $\mathrm{CA}$ \\
\hline 59 & Autauga & 1 & $\mathrm{AL}$ \\
\hline 60 & Baldwin & 3 & $\mathrm{AL}$ \\
\hline 61 & Barbour & 5 & $\mathrm{AL}$ \\
\hline 62 & Bibb & 7 & $\mathrm{AL}$ \\
\hline 63 & Blount & 9 & $\mathrm{AL}$ \\
\hline 64 & Bullock & 11 & $\mathrm{AL}$ \\
\hline 65 & Butler & 13 & $\mathrm{AL}$ \\
\hline 66 & Calhoun & 15 & $\mathrm{AL}$ \\
\hline 67 & Chambers & 17 & $\mathrm{AL}$ \\
\hline 68 & Cherokee & 19 & $\mathrm{AL}$ \\
\hline 69 & Chilton & 21 & $\mathrm{AL}$ \\
\hline 70 & Choctaw & 23 & $\mathrm{AL}$ \\
\hline 71 & Clarke & 25 & $\mathrm{AL}$ \\
\hline 72 & Clay & 27 & $\mathrm{AL}$ \\
\hline 73 & Cleburne & 29 & $\mathrm{AL}$ \\
\hline 74 & Coffee & 31 & $\mathrm{AL}$ \\
\hline 75 & Colbert & 33 & $\mathrm{AL}$ \\
\hline 76 & Conecuh & 35 & $\mathrm{AL}$ \\
\hline 77 & Coosa & 37 & $\mathrm{AL}$ \\
\hline 78 & Covington & 39 & $\mathrm{AL}$ \\
\hline 79 & Crenshaw & 41 & $\mathrm{AL}$ \\
\hline 80 & Cullman & 43 & $\mathrm{AL}$ \\
\hline 81 & Dale & 45 & $\mathrm{AL}$ \\
\hline 82 & Dallas & 47 & $\mathrm{AL}$ \\
\hline 83 & DeKalb & 49 & $\mathrm{AL}$ \\
\hline 84 & Elmore & 51 & $\mathrm{AL}$ \\
\hline 85 & Escambia & 53 & $\mathrm{AL}$ \\
\hline 86 & Etowah & 55 & $\mathrm{AL}$ \\
\hline 87 & Fayette & 57 & $\mathrm{AL}$ \\
\hline 88 & Franklin & 59 & $\mathrm{AL}$ \\
\hline
\end{tabular}


luCounty-Continued

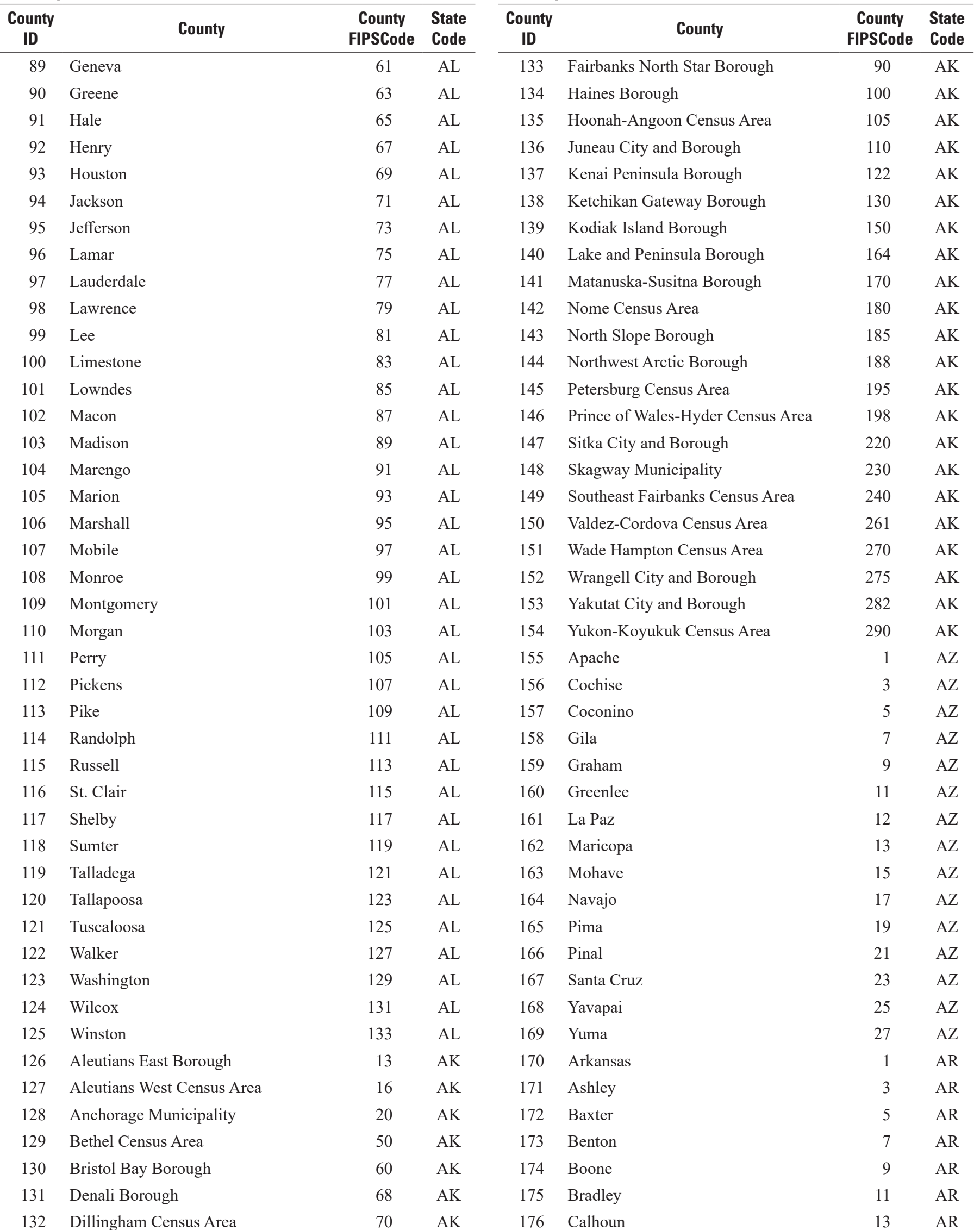


luCounty-Continued

\begin{tabular}{|c|c|c|c|c|c|c|c|}
\hline $\begin{array}{c}\text { County } \\
\text { ID }\end{array}$ & County & $\begin{array}{l}\text { County } \\
\text { FIPSCode }\end{array}$ & $\begin{array}{l}\text { State } \\
\text { Code }\end{array}$ & $\begin{array}{c}\text { County } \\
\text { ID }\end{array}$ & County & $\begin{array}{l}\text { County } \\
\text { FIPSCode }\end{array}$ & $\begin{array}{l}\text { State } \\
\text { Code }\end{array}$ \\
\hline 177 & Carroll & 15 & $\mathrm{AR}$ & 221 & Ouachita & 103 & AR \\
\hline 179 & Clark & 19 & $\mathrm{AR}$ & 223 & Phillips & 107 & $\mathrm{AR}$ \\
\hline 180 & Clay & 21 & $\mathrm{AR}$ & 224 & Pike & 109 & AR \\
\hline 183 & Columbia & 27 & $\mathrm{AR}$ & 227 & Pope & 115 & AR \\
\hline 184 & Conway & 29 & $\mathrm{AR}$ & 228 & Prairie & 117 & AR \\
\hline 185 & Craighead & 31 & $\mathrm{AR}$ & 229 & Pulaski & 119 & $\mathrm{AR}$ \\
\hline 186 & Crawford & 33 & $\mathrm{AR}$ & 230 & Randolph & 121 & $\mathrm{AR}$ \\
\hline 190 & Desha & 41 & $\mathrm{AR}$ & 234 & Searcy & 129 & $\mathrm{AR}$ \\
\hline 191 & Drew & 43 & $\mathrm{AR}$ & 235 & Sebastian & 131 & AR \\
\hline 192 & Faulkner & 45 & $\mathrm{AR}$ & 236 & Sevier & 133 & $\mathrm{AR}$ \\
\hline 193 & Franklin & 47 & $\mathrm{AR}$ & 237 & Sharp & 135 & $\mathrm{AR}$ \\
\hline 194 & Fulton & 49 & $\mathrm{AR}$ & 238 & Stone & 137 & AR \\
\hline 195 & Garland & 51 & $\mathrm{AR}$ & 239 & Union & 139 & AR \\
\hline 196 & Grant & 53 & $\mathrm{AR}$ & 240 & Van Buren & 141 & AR \\
\hline 197 & Greene & 55 & $\mathrm{AR}$ & 241 & Washington & 143 & $\mathrm{AR}$ \\
\hline 198 & Hempstead & 57 & $\mathrm{AR}$ & 242 & White & 145 & $\mathrm{AR}$ \\
\hline 204 & Jefferson & 69 & $\mathrm{AR}$ & 306 & Archuleta & 7 & $\mathrm{CO}$ \\
\hline 205 & Johnson & 71 & AR & 307 & Baca & 9 & $\mathrm{CO}$ \\
\hline 206 & Lafayette & 73 & $\mathrm{AR}$ & 308 & Bent & 11 & $\mathrm{CO}$ \\
\hline 207 & Lawrence & 75 & $\mathrm{AR}$ & 309 & Boulder & 13 & $\mathrm{CO}$ \\
\hline 208 & Lee & 77 & $\mathrm{AR}$ & 310 & Broomfield & 14 & $\mathrm{CO}$ \\
\hline 209 & Lincoln & 79 & $\mathrm{AR}$ & 311 & Chaffee & 15 & $\mathrm{CO}$ \\
\hline 210 & Little River & 81 & $\mathrm{AR}$ & 312 & Cheyenne & 17 & $\mathrm{CO}$ \\
\hline 211 & Logan & 83 & $\mathrm{AR}$ & 313 & Clear Creek & 19 & $\mathrm{CO}$ \\
\hline 212 & Lonoke & 85 & $\mathrm{AR}$ & 314 & Conejos & 21 & $\mathrm{CO}$ \\
\hline 213 & Madison & 87 & $\mathrm{AR}$ & 315 & Costilla & 23 & $\mathrm{CO}$ \\
\hline 214 & Marion & 89 & $\mathrm{AR}$ & 316 & Crowley & 25 & $\mathrm{CO}$ \\
\hline 215 & Miller & 91 & $\mathrm{AR}$ & 317 & Custer & 27 & $\mathrm{CO}$ \\
\hline 216 & Mississippi & 93 & AR & 318 & Delta & 29 & $\mathrm{CO}$ \\
\hline 217 & Monroe & 95 & $\mathrm{AR}$ & 319 & Denver & 31 & $\mathrm{CO}$ \\
\hline 218 & Montgomery & 97 & $\mathrm{AR}$ & 320 & Dolores & 33 & $\mathrm{CO}$ \\
\hline 219 & Nevada & 99 & $\mathrm{AR}$ & 321 & Douglas & 35 & $\mathrm{CO}$ \\
\hline 220 & Newton & 101 & $\mathrm{AR}$ & 322 & Eagle & 37 & $\mathrm{CO}$ \\
\hline
\end{tabular}


luCounty-Continued

\begin{tabular}{|c|c|c|c|c|c|c|c|}
\hline $\begin{array}{l}\text { County } \\
\text { ID }\end{array}$ & County & $\begin{array}{l}\text { County } \\
\text { FIPSCode }\end{array}$ & $\begin{array}{l}\text { State } \\
\text { Code }\end{array}$ & $\begin{array}{c}\text { County } \\
\text { ID }\end{array}$ & County & $\begin{array}{l}\text { County } \\
\text { FIPSCode }\end{array}$ & $\begin{array}{l}\text { State } \\
\text { Code }\end{array}$ \\
\hline 323 & Elbert & 39 & $\mathrm{CO}$ & 367 & Fairfield & 1 & $\mathrm{CT}$ \\
\hline 325 & Fremont & 43 & $\mathrm{CO}$ & 369 & Litchfield & 5 & $\mathrm{CT}$ \\
\hline 326 & Garfield & 45 & $\mathrm{CO}$ & 370 & Middlesex & 7 & $\mathrm{CT}$ \\
\hline 329 & Gunnison & 51 & $\mathrm{CO}$ & 373 & Tolland & 13 & $\mathrm{CT}$ \\
\hline 330 & Hinsdale & 53 & $\mathrm{CO}$ & 374 & Windham & 15 & $\mathrm{CT}$ \\
\hline 331 & Huerfano & 55 & $\mathrm{CO}$ & 375 & Kent & 1 & $\mathrm{DE}$ \\
\hline 332 & Jackson & 57 & $\mathrm{CO}$ & 376 & New Castle & 3 & $\mathrm{DE}$ \\
\hline 336 & Lake & 65 & $\mathrm{CO}$ & 380 & Baker & 3 & FL \\
\hline 337 & La Plata & 67 & $\mathrm{CO}$ & 381 & Bay & 5 & FL \\
\hline 338 & Larimer & 69 & $\mathrm{CO}$ & 382 & Bradford & 7 & $\mathrm{FL}$ \\
\hline 339 & Las Animas & 71 & $\mathrm{CO}$ & 383 & Brevard & 9 & $\mathrm{FL}$ \\
\hline 340 & Lincoln & 73 & $\mathrm{CO}$ & 384 & Broward & 11 & $\mathrm{FL}$ \\
\hline 341 & Logan & 75 & $\mathrm{CO}$ & 385 & Calhoun & 13 & FL \\
\hline 342 & Mesa & 77 & $\mathrm{CO}$ & 386 & Charlotte & 15 & $\mathrm{FL}$ \\
\hline 343 & Mineral & 79 & $\mathrm{CO}$ & 387 & Citrus & 17 & $\mathrm{FL}$ \\
\hline 344 & Moffat & 81 & $\mathrm{CO}$ & 388 & Clay & 19 & $\mathrm{FL}$ \\
\hline 351 & Phillips & 95 & $\mathrm{CO}$ & 395 & Flagler & 35 & $\mathrm{FL}$ \\
\hline 352 & Pitkin & 97 & $\mathrm{CO}$ & 396 & Franklin & 37 & $\mathrm{FL}$ \\
\hline 353 & Prowers & 99 & $\mathrm{CO}$ & 397 & Gadsden & 39 & FL \\
\hline 354 & Pueblo & 101 & $\mathrm{CO}$ & 398 & Gilchrist & 41 & $\mathrm{FL}$ \\
\hline 355 & Rio Blanco & 103 & $\mathrm{CO}$ & 399 & Glades & 43 & $\mathrm{FL}$ \\
\hline 356 & Rio Grande & 105 & $\mathrm{CO}$ & 400 & Gulf & 45 & $\mathrm{FL}$ \\
\hline 357 & Routt & 107 & $\mathrm{CO}$ & 401 & Hamilton & 47 & $\mathrm{FL}$ \\
\hline 358 & Saguache & 109 & $\mathrm{CO}$ & 402 & Hardee & 49 & FL \\
\hline 359 & San Juan & 111 & $\mathrm{CO}$ & 403 & Hendry & 51 & FL \\
\hline 360 & San Miguel & 113 & $\mathrm{CO}$ & 404 & Hernando & 53 & $\mathrm{FL}$ \\
\hline 361 & Sedgwick & 115 & $\mathrm{CO}$ & 405 & Highlands & 55 & $\mathrm{FL}$ \\
\hline 362 & Summit & 117 & $\mathrm{CO}$ & 406 & Hillsborough & 57 & $\mathrm{FL}$ \\
\hline 363 & Teller & 119 & $\mathrm{CO}$ & 407 & Holmes & 59 & $\mathrm{FL}$ \\
\hline 364 & Washington & 121 & $\mathrm{CO}$ & 408 & Indian River & 61 & FL \\
\hline 365 & Weld & 123 & $\mathrm{CO}$ & 409 & Jackson & 63 & $\mathrm{FL}$ \\
\hline 366 & Yuma & 125 & $\mathrm{CO}$ & 410 & Jefferson & 65 & $\mathrm{FL}$ \\
\hline
\end{tabular}


luCounty-Continued

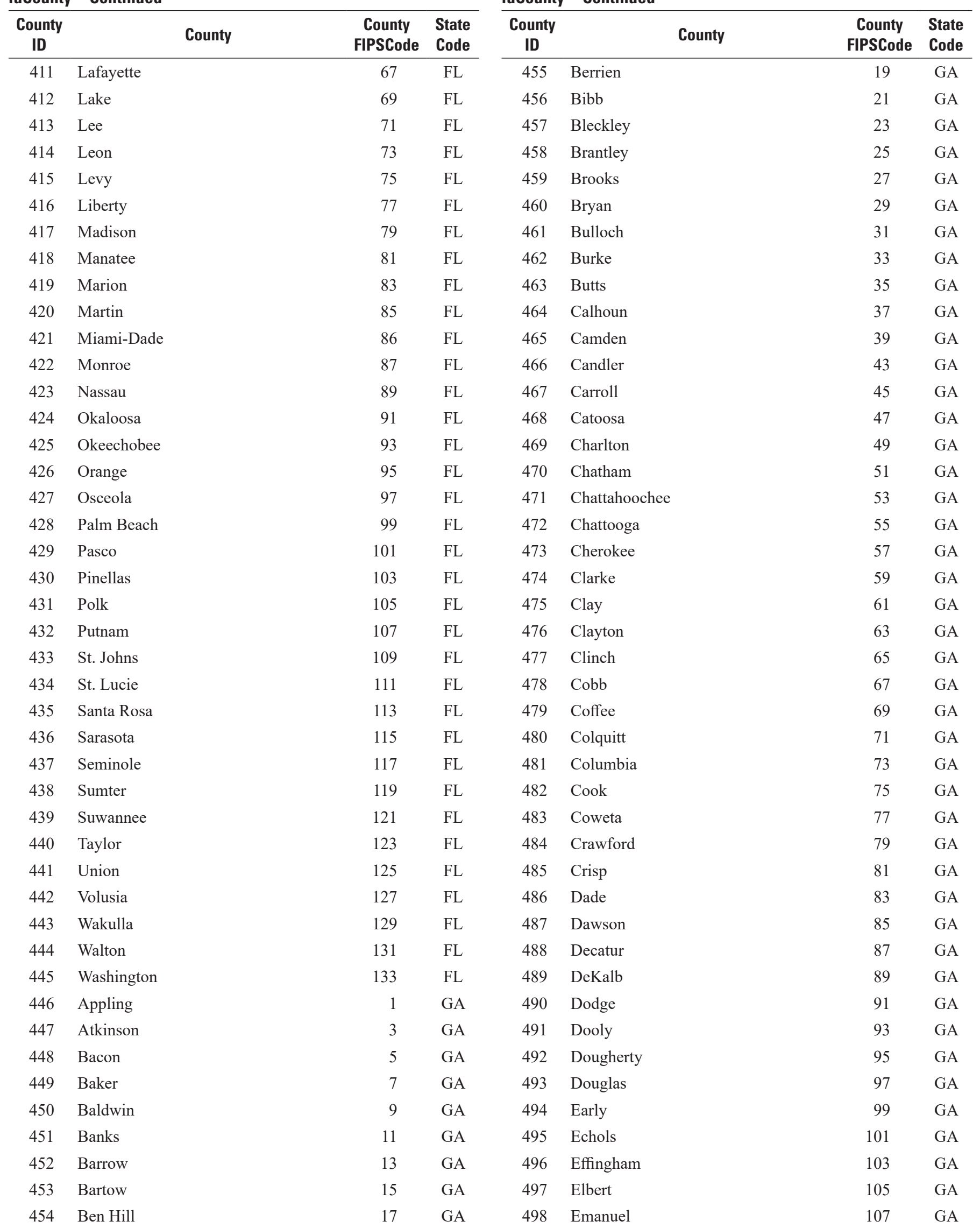


luCounty-Continued

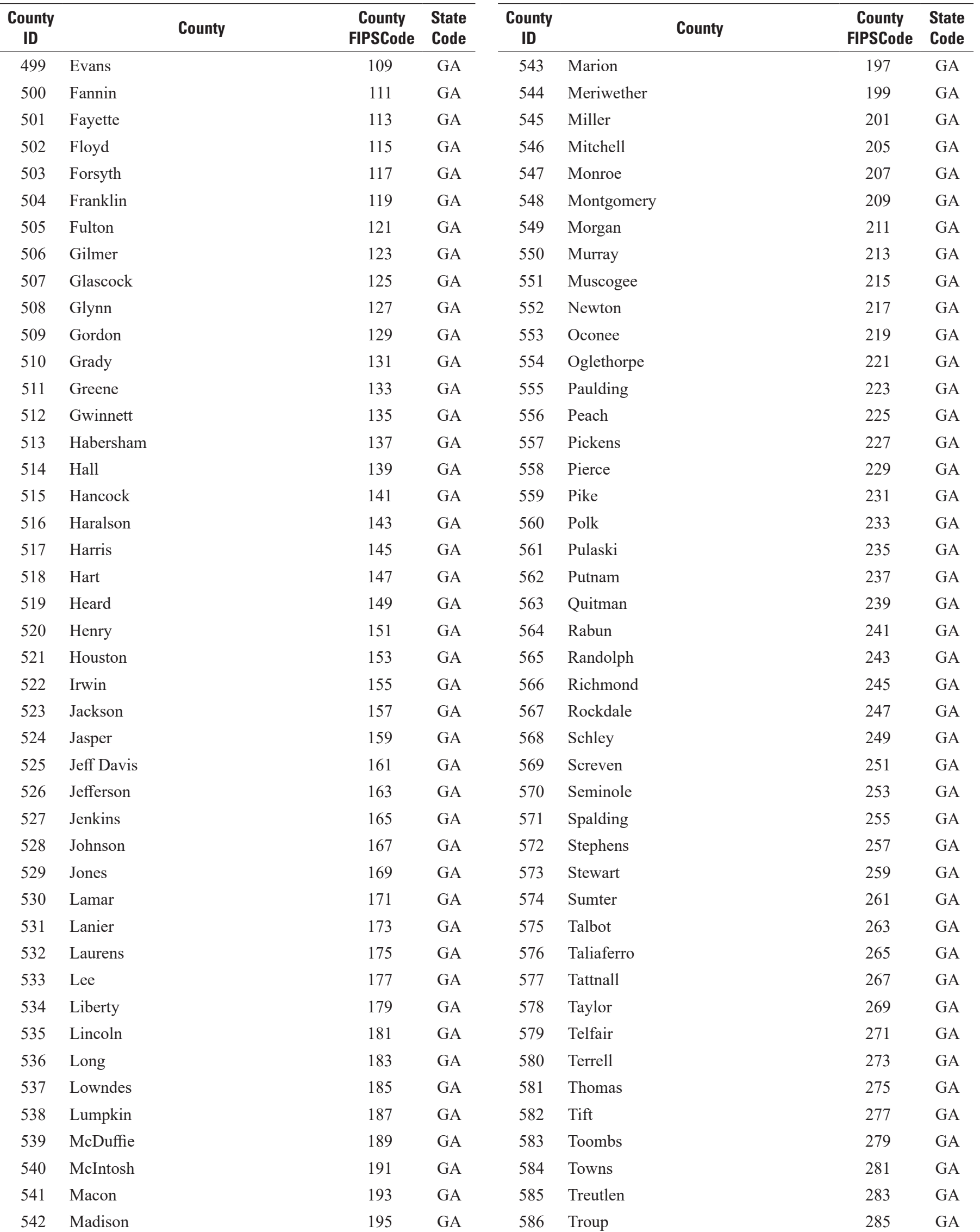


luCounty-Continued

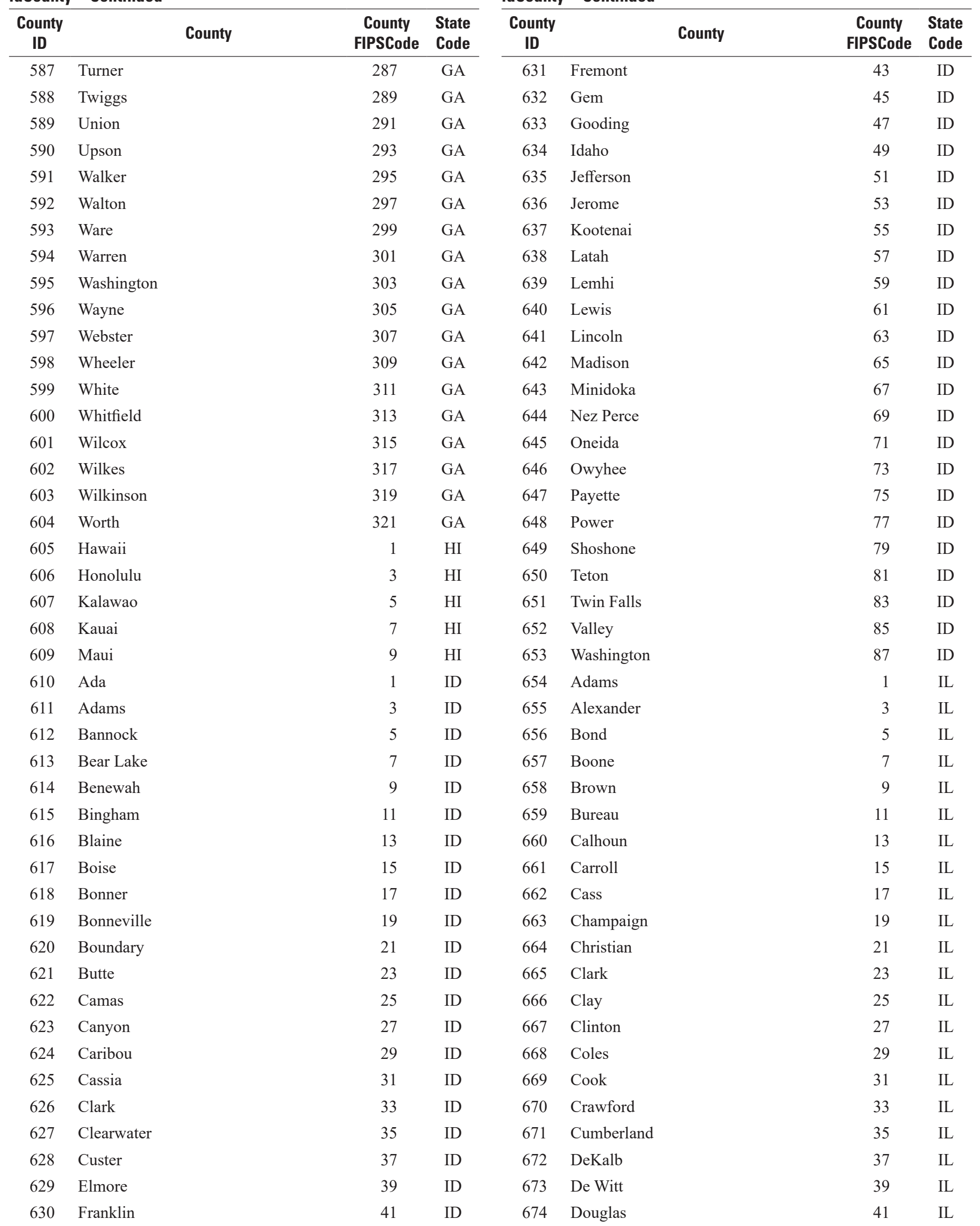


luCounty-Continued

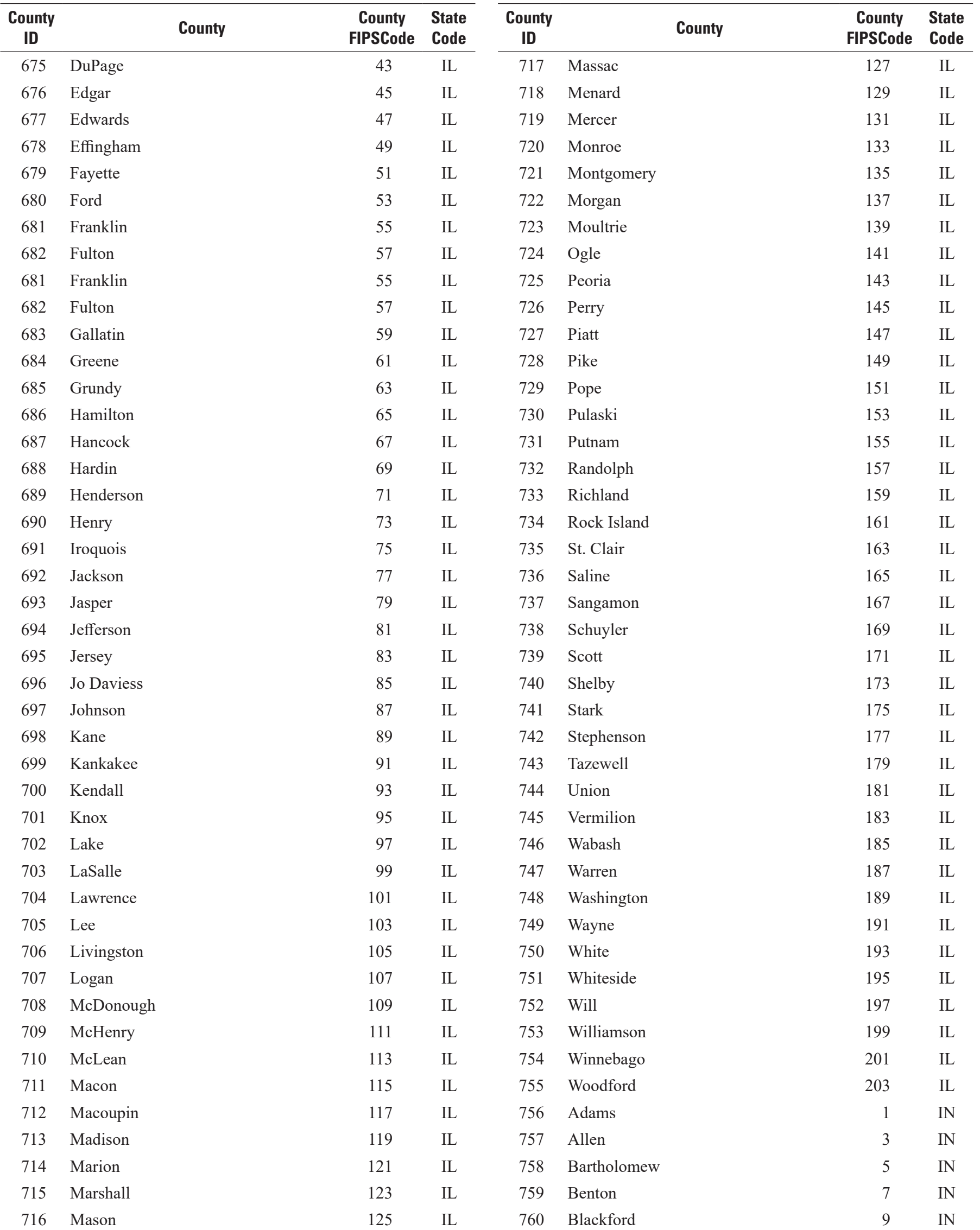


luCounty-Continued

\begin{tabular}{|c|c|c|c|c|c|c|c|}
\hline $\begin{array}{l}\text { County } \\
\text { ID }\end{array}$ & County & $\begin{array}{l}\text { County } \\
\text { FIPSCode }\end{array}$ & $\begin{array}{l}\text { State } \\
\text { Code }\end{array}$ & $\begin{array}{c}\text { County } \\
\text { ID }\end{array}$ & County & $\begin{array}{l}\text { County } \\
\text { FIPSCode }\end{array}$ & $\begin{array}{l}\text { State } \\
\text { Code }\end{array}$ \\
\hline 761 & Boone & 11 & IN & 805 & Marshall & 99 & IN \\
\hline 763 & Carroll & 15 & IN & 807 & Miami & 103 & IN \\
\hline 764 & Cass & 17 & IN & 808 & Monroe & 105 & IN \\
\hline 767 & Clinton & 23 & IN & 811 & Newton & 111 & IN \\
\hline 768 & Crawford & 25 & IN & 812 & Noble & 113 & IN \\
\hline 769 & Daviess & 27 & IN & 813 & Ohio & 115 & IN \\
\hline 770 & Dearborn & 29 & IN & 814 & Orange & 117 & IN \\
\hline 774 & Dubois & 37 & IN & 818 & Pike & 125 & IN \\
\hline 775 & Elkhart & 39 & IN & 819 & Porter & 127 & IN \\
\hline 776 & Fayette & 41 & IN & 820 & Posey & 129 & IN \\
\hline 777 & Floyd & 43 & IN & 821 & Pulaski & 131 & IN \\
\hline 778 & Fountain & 45 & IN & 822 & Putnam & 133 & IN \\
\hline 779 & Franklin & 47 & IN & 823 & Randolph & 135 & IN \\
\hline 780 & Fulton & 49 & IN & 824 & Ripley & 137 & IN \\
\hline 781 & Gibson & 51 & $\mathrm{IN}$ & 825 & Rush & 139 & IN \\
\hline 782 & Grant & 53 & IN & 826 & St. Joseph & 141 & IN \\
\hline 788 & Henry & 65 & IN & 832 & Sullivan & 153 & IN \\
\hline 789 & Howard & 67 & IN & 833 & Switzerland & 155 & IN \\
\hline 790 & Huntington & 69 & IN & 834 & Tippecanoe & 157 & IN \\
\hline 791 & Jackson & 71 & IN & 835 & Tipton & 159 & IN \\
\hline 792 & Jasper & 73 & IN & 836 & Union & 161 & IN \\
\hline 793 & Jay & 75 & IN & 837 & Vanderburgh & 163 & IN \\
\hline 794 & Jefferson & 77 & IN & 838 & Vermillion & 165 & IN \\
\hline 795 & Jennings & 79 & IN & 839 & Vigo & 167 & IN \\
\hline 796 & Johnson & 81 & IN & 840 & Wabash & 169 & IN \\
\hline 797 & Knox & 83 & IN & 841 & Warren & 171 & IN \\
\hline 798 & Kosciusko & 85 & IN & 842 & Warrick & 173 & IN \\
\hline 799 & LaGrange & 87 & IN & 843 & Washington & 175 & IN \\
\hline 800 & Lake & 89 & IN & 844 & Wayne & 177 & IN \\
\hline 801 & LaPorte & 91 & IN & 845 & Wells & 179 & IN \\
\hline 802 & Lawrence & 93 & IN & 846 & White & 181 & IN \\
\hline 803 & Madison & 95 & IN & 847 & Whitley & 183 & IN \\
\hline 804 & Marion & 97 & IN & 848 & Adair & 1 & IA \\
\hline
\end{tabular}


luCounty-Continued

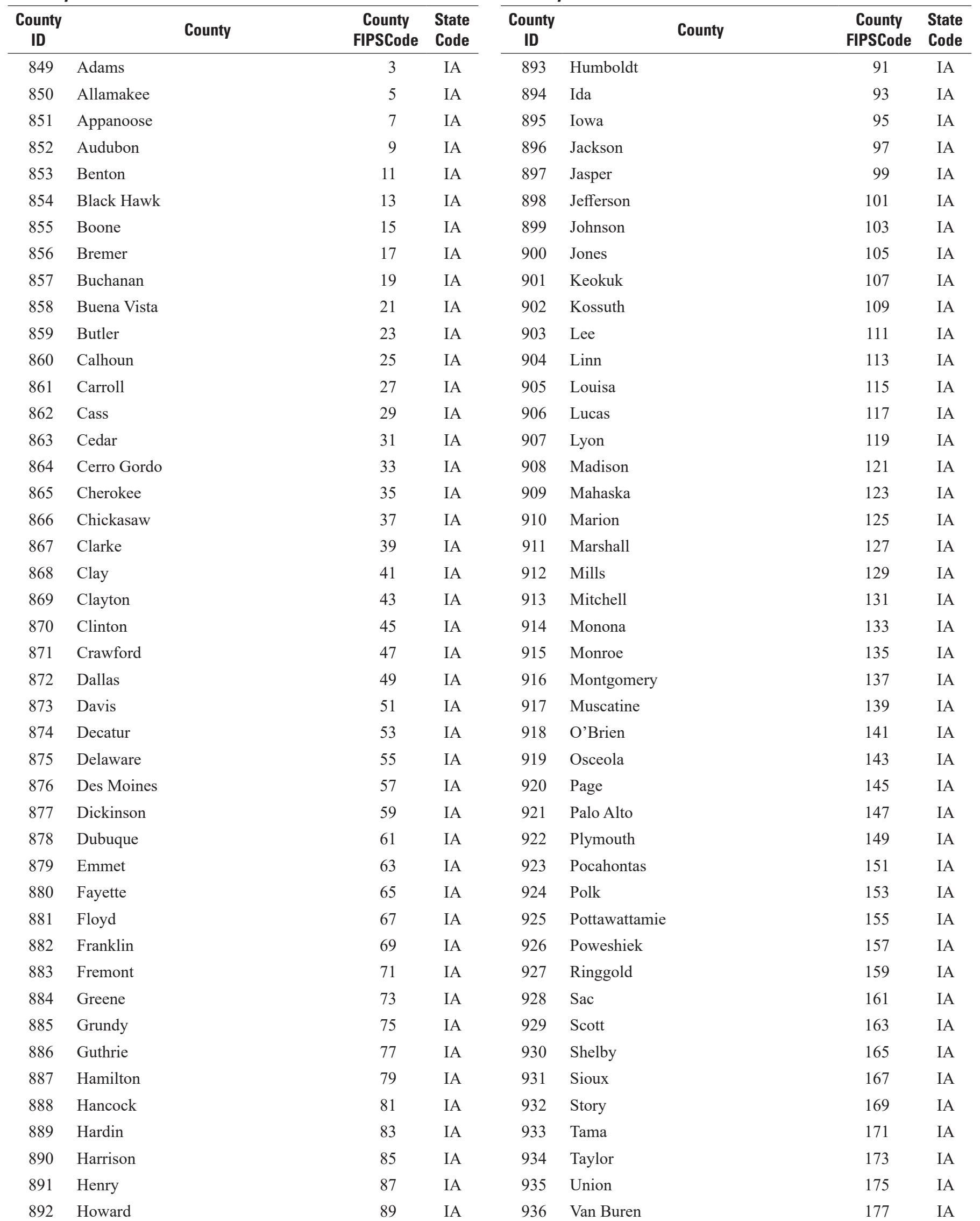


luCounty-Continued

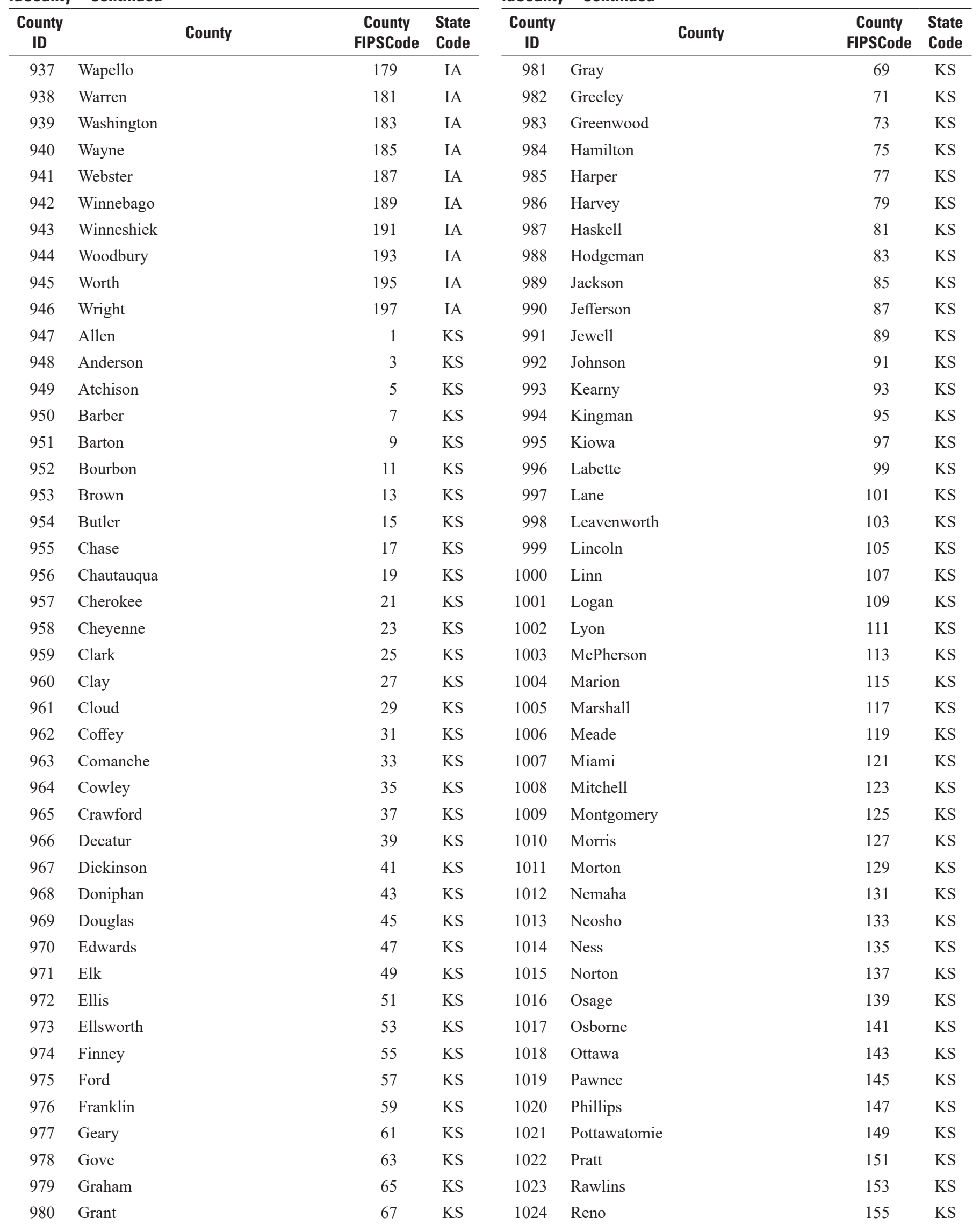


luCounty-Continued

\begin{tabular}{|c|c|c|c|c|c|c|c|}
\hline $\begin{array}{c}\text { County } \\
\text { ID }\end{array}$ & County & $\begin{array}{c}\text { County } \\
\text { FIPSCode }\end{array}$ & $\begin{array}{l}\text { State } \\
\text { Code }\end{array}$ & $\begin{array}{c}\text { County } \\
\text { ID }\end{array}$ & County & $\begin{array}{l}\text { County } \\
\text { FIPSCode }\end{array}$ & $\begin{array}{l}\text { State } \\
\text { Code }\end{array}$ \\
\hline 1025 & Republic & 157 & $\mathrm{KS}$ & 1069 & Calloway & 35 & KY \\
\hline 1026 & Rice & 159 & $\mathrm{KS}$ & 1070 & Campbell & 37 & KY \\
\hline 1027 & Riley & 161 & $\mathrm{KS}$ & 1071 & Carlisle & 39 & KY \\
\hline 1028 & Rooks & 163 & $\mathrm{KS}$ & 1072 & Carroll & 41 & $\mathrm{KY}$ \\
\hline 1029 & Rush & 165 & $\mathrm{KS}$ & 1073 & Carter & 43 & KY \\
\hline 1030 & Russell & 167 & $\mathrm{KS}$ & 1074 & Casey & 45 & KY \\
\hline 1031 & Saline & 169 & $\mathrm{KS}$ & 1075 & Christian & 47 & $\mathrm{KY}$ \\
\hline 1032 & Scott & 171 & $\mathrm{KS}$ & 1076 & Clark & 49 & KY \\
\hline 1033 & Sedgwick & 173 & $\mathrm{KS}$ & 1077 & Clay & 51 & KY \\
\hline 1034 & Seward & 175 & $\mathrm{KS}$ & 1078 & Clinton & 53 & $\mathrm{KY}$ \\
\hline 1035 & Shawnee & 177 & $\mathrm{KS}$ & 1079 & Crittenden & 55 & KY \\
\hline 1036 & Sheridan & 179 & $\mathrm{KS}$ & 1080 & Cumberland & 57 & KY \\
\hline 1037 & Sherman & 181 & $\mathrm{KS}$ & 1081 & Daviess & 59 & KY \\
\hline 1038 & Smith & 183 & $\mathrm{KS}$ & 1082 & Edmonson & 61 & KY \\
\hline 1039 & Stafford & 185 & $\mathrm{KS}$ & 1083 & Elliott & 63 & KY \\
\hline 1040 & Stanton & 187 & $\mathrm{KS}$ & 1084 & Estill & 65 & KY \\
\hline 1041 & Stevens & 189 & $\mathrm{KS}$ & 1085 & Fayette & 67 & KY \\
\hline 1042 & Sumner & 191 & $\mathrm{KS}$ & 1086 & Fleming & 69 & KY \\
\hline 1043 & Thomas & 193 & $\mathrm{KS}$ & 1087 & Floyd & 71 & $\mathrm{KY}$ \\
\hline 1044 & Trego & 195 & $\mathrm{KS}$ & 1088 & Franklin & 73 & KY \\
\hline 1045 & Wabaunsee & 197 & $\mathrm{KS}$ & 1089 & Fulton & 75 & $\mathrm{KY}$ \\
\hline 1046 & Wallace & 199 & $\mathrm{KS}$ & 1090 & Gallatin & 77 & $\mathrm{KY}$ \\
\hline 1047 & Washington & 201 & $\mathrm{KS}$ & 1091 & Garrard & 79 & KY \\
\hline 1048 & Wichita & 203 & $\mathrm{KS}$ & 1092 & Grant & 81 & $\mathrm{KY}$ \\
\hline 1049 & Wilson & 205 & $\mathrm{KS}$ & 1093 & Graves & 83 & KY \\
\hline 1050 & Woodson & 207 & $\mathrm{KS}$ & 1094 & Grayson & 85 & KY \\
\hline 1051 & Wyandotte & 209 & $\mathrm{KS}$ & 1095 & Green & 87 & $\mathrm{KY}$ \\
\hline 1052 & Adair & 1 & KY & 1096 & Greenup & 89 & KY \\
\hline 1053 & Allen & 3 & KY & 1097 & Hancock & 91 & KY \\
\hline 1054 & Anderson & 5 & $\mathrm{KY}$ & 1098 & Hardin & 93 & KY \\
\hline 1055 & Ballard & 7 & KY & 1099 & Harlan & 95 & KY \\
\hline 1056 & Barren & 9 & $\mathrm{KY}$ & 1100 & Harrison & 97 & KY \\
\hline 1057 & Bath & 11 & $\mathrm{KY}$ & 1101 & Hart & 99 & KY \\
\hline 1058 & Bell & 13 & $\mathrm{KY}$ & 1102 & Henderson & 101 & $\mathrm{KY}$ \\
\hline 1059 & Boone & 15 & KY & 1103 & Henry & 103 & KY \\
\hline 1060 & Bourbon & 17 & KY & 1104 & Hickman & 105 & KY \\
\hline 1061 & Boyd & 19 & KY & 1105 & Hopkins & 107 & KY \\
\hline 1062 & Boyle & 21 & $\mathrm{KY}$ & 1106 & Jackson & 109 & $\mathrm{KY}$ \\
\hline 1063 & Bracken & 23 & $\mathrm{KY}$ & 1107 & Jefferson & 111 & $\mathrm{KY}$ \\
\hline 1064 & Breathitt & 25 & $\mathrm{KY}$ & 1108 & Jessamine & 113 & KY \\
\hline 1065 & Breckinridge & 27 & KY & 1109 & Johnson & 115 & KY \\
\hline 1066 & Bullitt & 29 & KY & 1110 & Kenton & 117 & KY \\
\hline 1067 & Butler & 31 & $\mathrm{KY}$ & 1111 & Knott & 119 & KY \\
\hline 1068 & Caldwell & 33 & $\mathrm{KY}$ & 1112 & Knox & 121 & KY \\
\hline
\end{tabular}

luCounty-Continued 
IuCounty-Continued

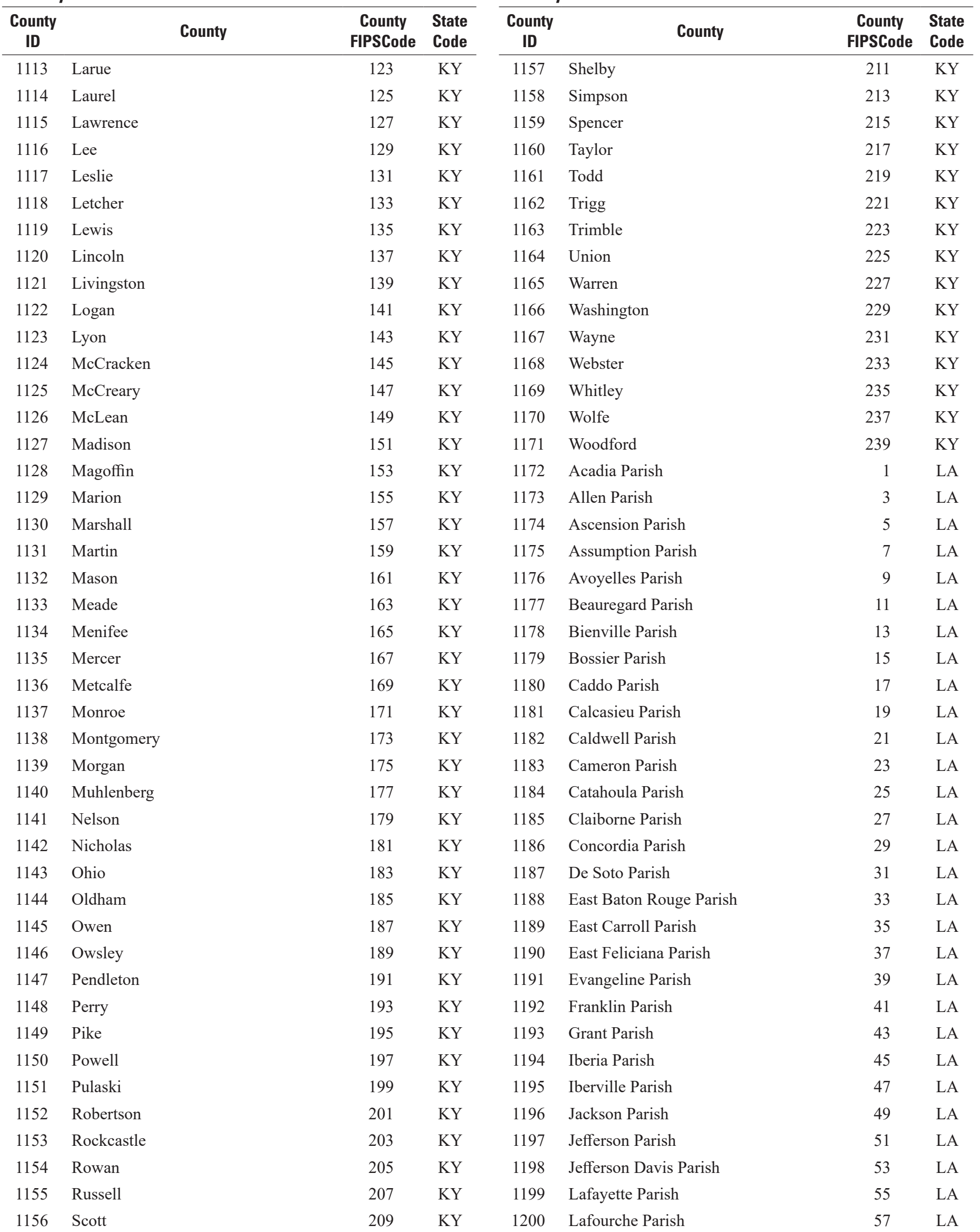




\begin{tabular}{|c|c|c|c|c|c|c|c|}
\hline \multicolumn{4}{|c|}{ luCounty-Continued } & \multicolumn{4}{|c|}{ luCounty-Continued } \\
\hline $\begin{array}{l}\text { County } \\
\text { ID }\end{array}$ & County & $\begin{array}{l}\text { County } \\
\text { FIPSCode }\end{array}$ & $\begin{array}{l}\text { State } \\
\text { Code }\end{array}$ & $\begin{array}{l}\text { County } \\
\text { ID }\end{array}$ & County & $\begin{array}{l}\text { County } \\
\text { FIPSCode }\end{array}$ & $\begin{array}{l}\text { State } \\
\text { Code }\end{array}$ \\
\hline 1201 & La Salle Parish & 59 & LA & 1245 & Penobscot & 19 & $\mathrm{ME}$ \\
\hline 1202 & Lincoln Parish & 61 & LA & 1246 & Piscataquis & 21 & $\mathrm{ME}$ \\
\hline 1203 & Livingston Parish & 63 & LA & 1247 & Sagadahoc & 23 & $\mathrm{ME}$ \\
\hline 1204 & Madison Parish & 65 & LA & 1248 & Somerset & 25 & $\mathrm{ME}$ \\
\hline 1205 & Morehouse Parish & 67 & LA & 1249 & Waldo & 27 & $\mathrm{ME}$ \\
\hline 1206 & Natchitoches Parish & 69 & LA & 1250 & Washington & 29 & $\mathrm{ME}$ \\
\hline 1207 & Orleans Parish & 71 & LA & 1251 & York & 31 & $\mathrm{ME}$ \\
\hline 1208 & Ouachita Parish & 73 & LA & 1252 & Allegany & 1 & MD \\
\hline 1209 & Plaquemines Parish & 75 & LA & 1253 & Anne Arundel & 3 & $\mathrm{MD}$ \\
\hline 1210 & Pointe Coupee Parish & 77 & LA & 1254 & Baltimore & 5 & MD \\
\hline 1211 & Rapides Parish & 79 & LA & 1255 & Calvert & 9 & $\mathrm{MD}$ \\
\hline 1212 & Red River Parish & 81 & LA & 1256 & Caroline & 11 & $\mathrm{MD}$ \\
\hline 1213 & Richland Parish & 83 & LA & 1257 & Carroll & 13 & $\mathrm{MD}$ \\
\hline 1214 & Sabine Parish & 85 & LA & 1258 & Cecil & 15 & MD \\
\hline 1215 & St. Bernard Parish & 87 & LA & 1259 & Charles & 17 & MD \\
\hline 1216 & St. Charles Parish & 89 & LA & 1260 & Dorchester & 19 & MD \\
\hline 1217 & St. Helena Parish & 91 & LA & 1261 & Frederick & 21 & MD \\
\hline 1218 & St. James Parish & 93 & LA & 1262 & Garrett & 23 & MD \\
\hline 1219 & St. John the Baptist Parish & 95 & LA & 1263 & Harford & 25 & $\mathrm{MD}$ \\
\hline 1220 & St. Landry Parish & 97 & LA & 1264 & Howard & 27 & MD \\
\hline 1221 & St. Martin Parish & 99 & LA & 1265 & Kent & 29 & MD \\
\hline 1222 & St. Mary Parish & 101 & LA & 1266 & Montgomery & 31 & $\mathrm{MD}$ \\
\hline 1223 & St. Tammany Parish & 103 & LA & 1267 & Prince George's & 33 & $\mathrm{MD}$ \\
\hline 1224 & Tangipahoa Parish & 105 & LA & 1268 & Queen Anne's & 35 & $\mathrm{MD}$ \\
\hline 1225 & Tensas Parish & 107 & LA & 1269 & St. Mary's & 37 & $\mathrm{MD}$ \\
\hline 1226 & Terrebonne Parish & 109 & LA & 1270 & Somerset & 39 & MD \\
\hline 1227 & Union Parish & 111 & LA & 1271 & Talbot & 41 & $\mathrm{MD}$ \\
\hline 1228 & Vermilion Parish & 113 & LA & 1272 & Washington & 43 & MD \\
\hline 1229 & Vernon Parish & 115 & LA & 1273 & Wicomico & 45 & MD \\
\hline 1230 & Washington Parish & 117 & LA & 1274 & Worcester & 47 & MD \\
\hline 1231 & Webster Parish & 119 & LA & 1275 & Baltimore city & 510 & MD \\
\hline 1232 & West Baton Rouge Parish & 121 & LA & 1276 & Barnstable & 1 & MA \\
\hline 1233 & West Carroll Parish & 123 & LA & 1277 & Berkshire & 3 & MA \\
\hline 1234 & West Feliciana Parish & 125 & LA & 1278 & Bristol & 5 & MA \\
\hline 1235 & Winn Parish & 127 & LA & 1279 & Dukes & 7 & MA \\
\hline 1236 & Androscoggin & 1 & $\mathrm{ME}$ & 1280 & Essex & 9 & MA \\
\hline 1237 & Aroostook & 3 & $\mathrm{ME}$ & 1281 & Franklin & 11 & MA \\
\hline 1238 & Cumberland & 5 & $\mathrm{ME}$ & 1282 & Hampden & 13 & MA \\
\hline 1239 & Franklin & 7 & $\mathrm{ME}$ & 1283 & Hampshire & 15 & MA \\
\hline 1240 & Hancock & 9 & $\mathrm{ME}$ & 1284 & Middlesex & 17 & MA \\
\hline 1241 & Kennebec & 11 & $\mathrm{ME}$ & 1285 & Nantucket & 19 & MA \\
\hline 1242 & Knox & 13 & $\mathrm{ME}$ & 1286 & Norfolk & 21 & MA \\
\hline 1243 & Lincoln & 15 & $\mathrm{ME}$ & 1287 & Plymouth & 23 & MA \\
\hline 1244 & Oxford & 17 & $\mathrm{ME}$ & 1288 & Suffolk & 25 & MA \\
\hline
\end{tabular}


luCounty-Continued

\begin{tabular}{|c|c|c|c|c|c|c|c|}
\hline $\begin{array}{c}\text { County } \\
\text { ID }\end{array}$ & County & $\begin{array}{l}\text { County } \\
\text { FIPSCode }\end{array}$ & $\begin{array}{l}\text { State } \\
\text { Code }\end{array}$ & $\begin{array}{c}\text { County } \\
\text { ID }\end{array}$ & County & $\begin{array}{l}\text { County } \\
\text { FIPSCode }\end{array}$ & $\begin{array}{l}\text { State } \\
\text { Code }\end{array}$ \\
\hline 1289 & Worcester & 27 & MA & 1334 & Leelanau & 89 & MI \\
\hline 1291 & Alger & 3 & MI & 1336 & Livingston & 93 & MI \\
\hline 1292 & Allegan & 5 & MI & 1337 & Luce & 95 & MI \\
\hline 1295 & Arenac & 11 & MI & 1340 & Manistee & 101 & MI \\
\hline 1296 & Baraga & 13 & MI & 1341 & Marquette & 103 & MI \\
\hline 1297 & Barry & 15 & MI & 1342 & Mason & 105 & MI \\
\hline 1298 & Bay & 17 & MI & 1343 & Mecosta & 107 & MI \\
\hline 1302 & Calhoun & 25 & MI & 1347 & Monroe & 115 & MI \\
\hline 1303 & Cass & 27 & MI & 1348 & Montcalm & 117 & MI \\
\hline 1304 & Charlevoix & 29 & MI & 1349 & Montmorency & 119 & MI \\
\hline 1305 & Cheboygan & 31 & MI & 1350 & Muskegon & 121 & MI \\
\hline 1306 & Chippewa & 33 & MI & 1351 & Newaygo & 123 & MI \\
\hline 1307 & Clare & 35 & MI & 1352 & Oakland & 125 & MI \\
\hline 1308 & Clinton & 37 & MI & 1353 & Oceana & 127 & MI \\
\hline 1309 & Crawford & 39 & MI & 1354 & Ogemaw & 129 & MI \\
\hline 1310 & Delta & 41 & MI & 1355 & Ontonagon & 131 & MI \\
\hline 1317 & Grand Traverse & 55 & MI & 1361 & Roscommon & 143 & MI \\
\hline 1318 & Gratiot & 57 & MI & 1362 & Saginaw & 145 & MI \\
\hline 1319 & Hillsdale & 59 & MI & 1363 & St. Clair & 147 & MI \\
\hline 1320 & Houghton & 61 & MI & 1364 & St. Joseph & 149 & MI \\
\hline 1321 & Huron & 63 & MI & 1365 & Sanilac & 151 & MI \\
\hline 1322 & Ingham & 65 & MI & 1366 & Schoolcraft & 153 & MI \\
\hline 1323 & Ionia & 67 & MI & 1367 & Shiawassee & 155 & MI \\
\hline 1324 & Iosco & 69 & MI & 1368 & Tuscola & 157 & MI \\
\hline 1325 & Iron & 71 & MI & 1369 & Van Buren & 159 & MI \\
\hline 1326 & Isabella & 73 & MI & 1370 & Washtenaw & 161 & MI \\
\hline 1327 & Jackson & 75 & MI & 1371 & Wayne & 163 & MI \\
\hline 1328 & Kalamazoo & 77 & MI & 1372 & Wexford & 165 & MI \\
\hline 1329 & Kalkaska & 79 & MI & 1373 & Aitkin & 1 & $\mathrm{MN}$ \\
\hline 1330 & Kent & 81 & MI & 1374 & Anoka & 3 & $\mathrm{MN}$ \\
\hline 1331 & Keweenaw & 83 & MI & 1375 & Becker & 5 & $\mathrm{MN}$ \\
\hline 1332 & Lake & 85 & MI & 1376 & Beltrami & 7 & $\mathrm{MN}$ \\
\hline 1333 & Lapeer & 87 & MI & 1377 & Benton & 9 & $\mathrm{MN}$ \\
\hline
\end{tabular}


luCounty-Continued

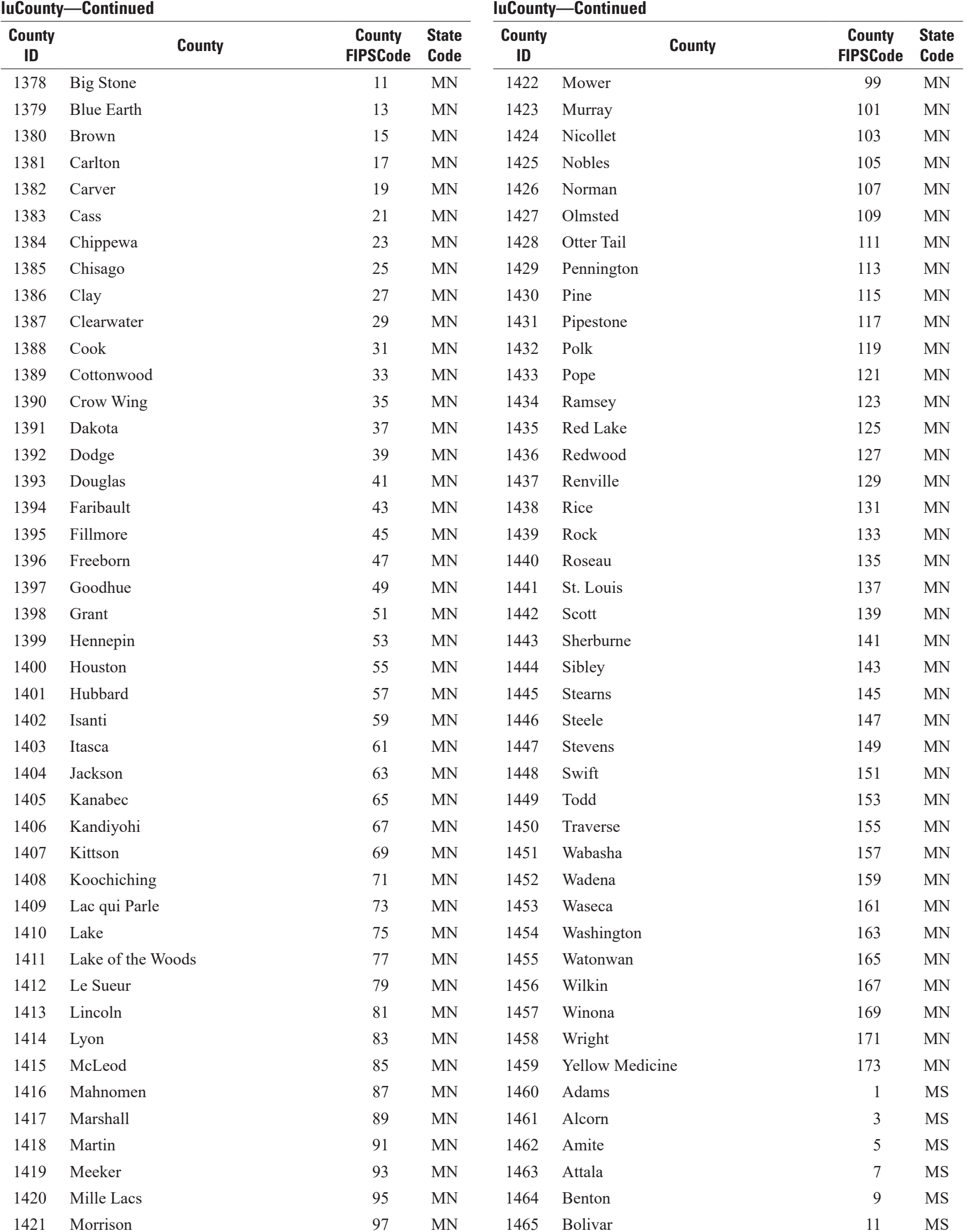

IuCounty-Continued 
luCounty-Continued

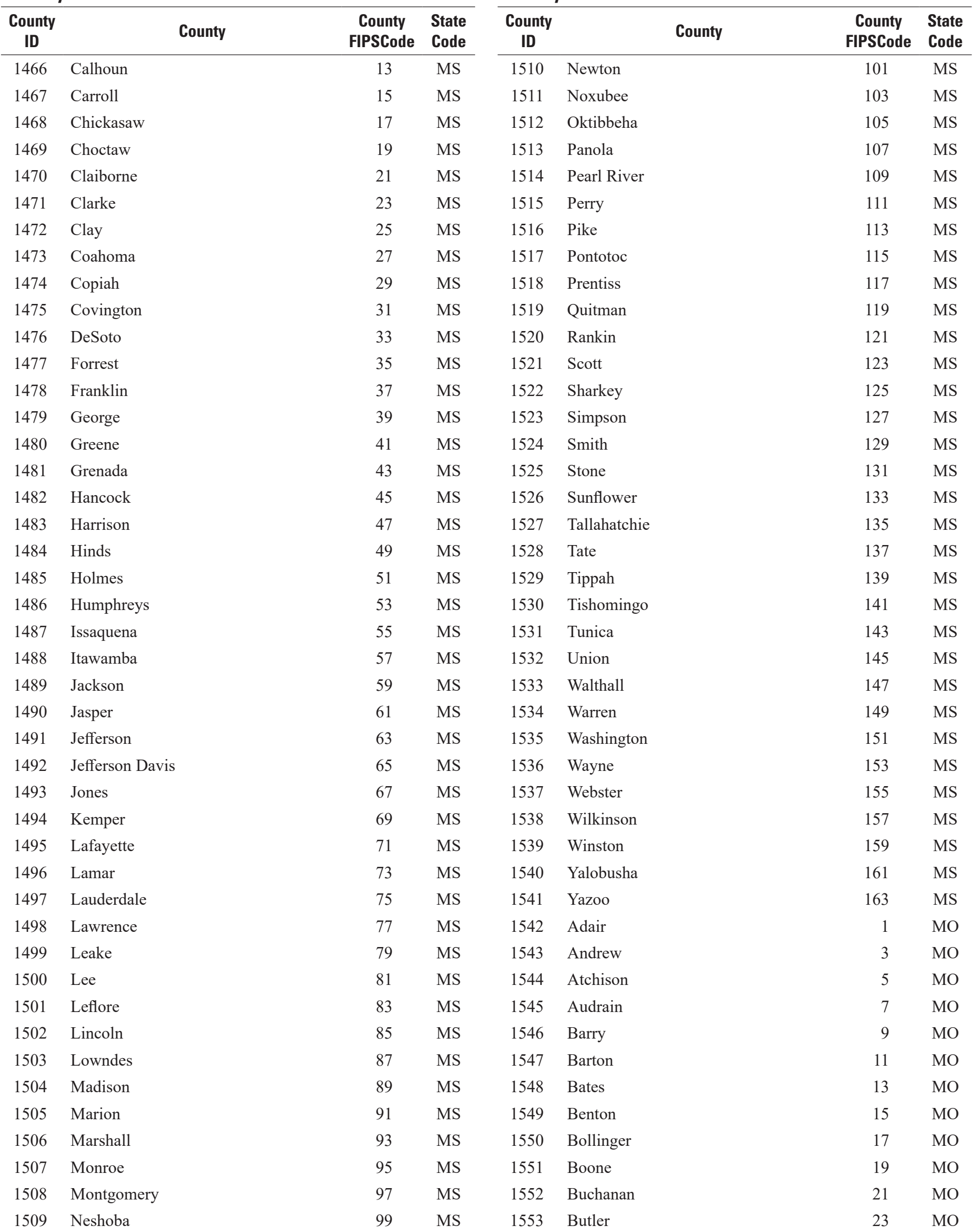


luCounty-Continued

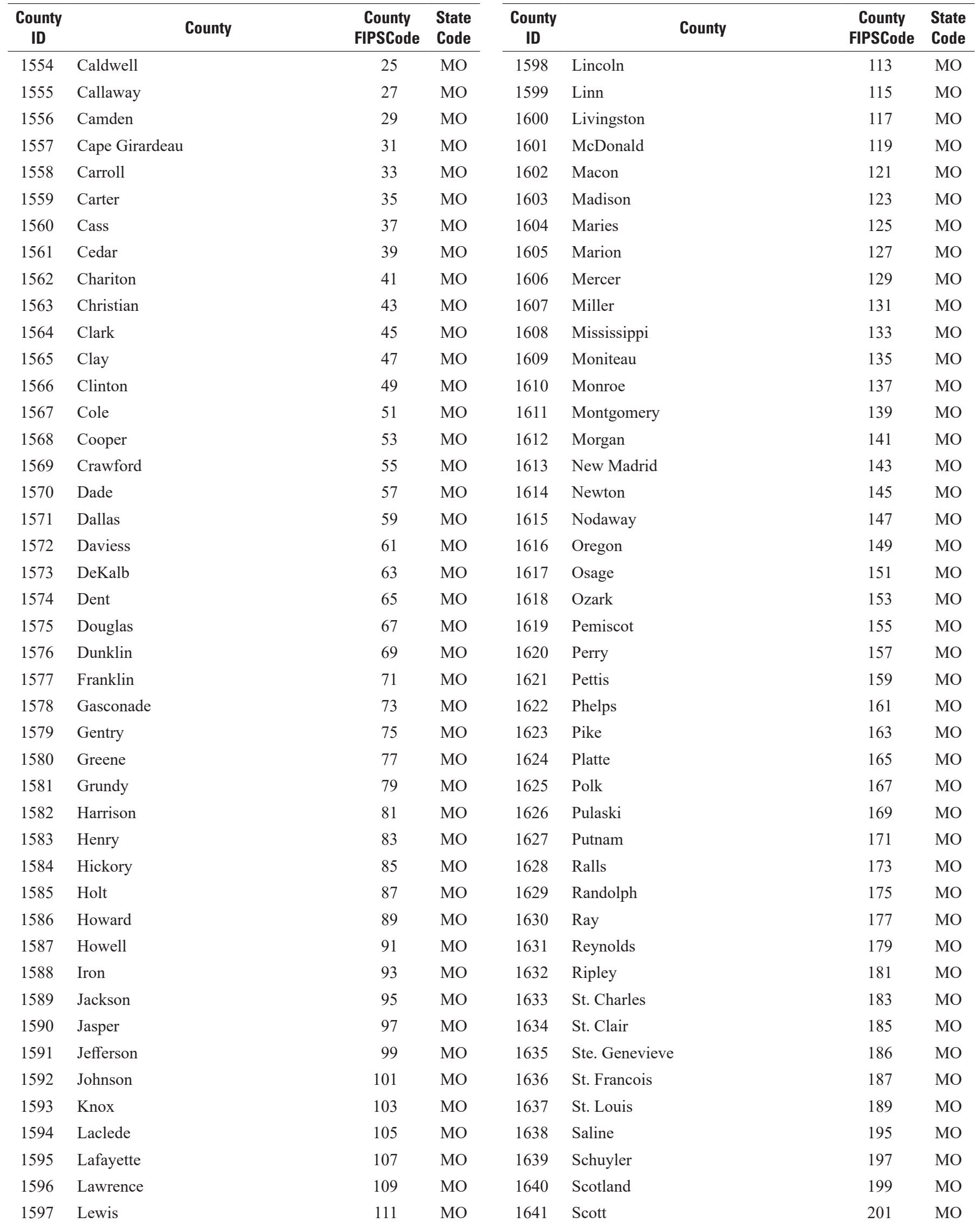

luCounty-Continued

ID

SECTION III:LOOK UP DOMAIN TABLES 
luCounty-Continued

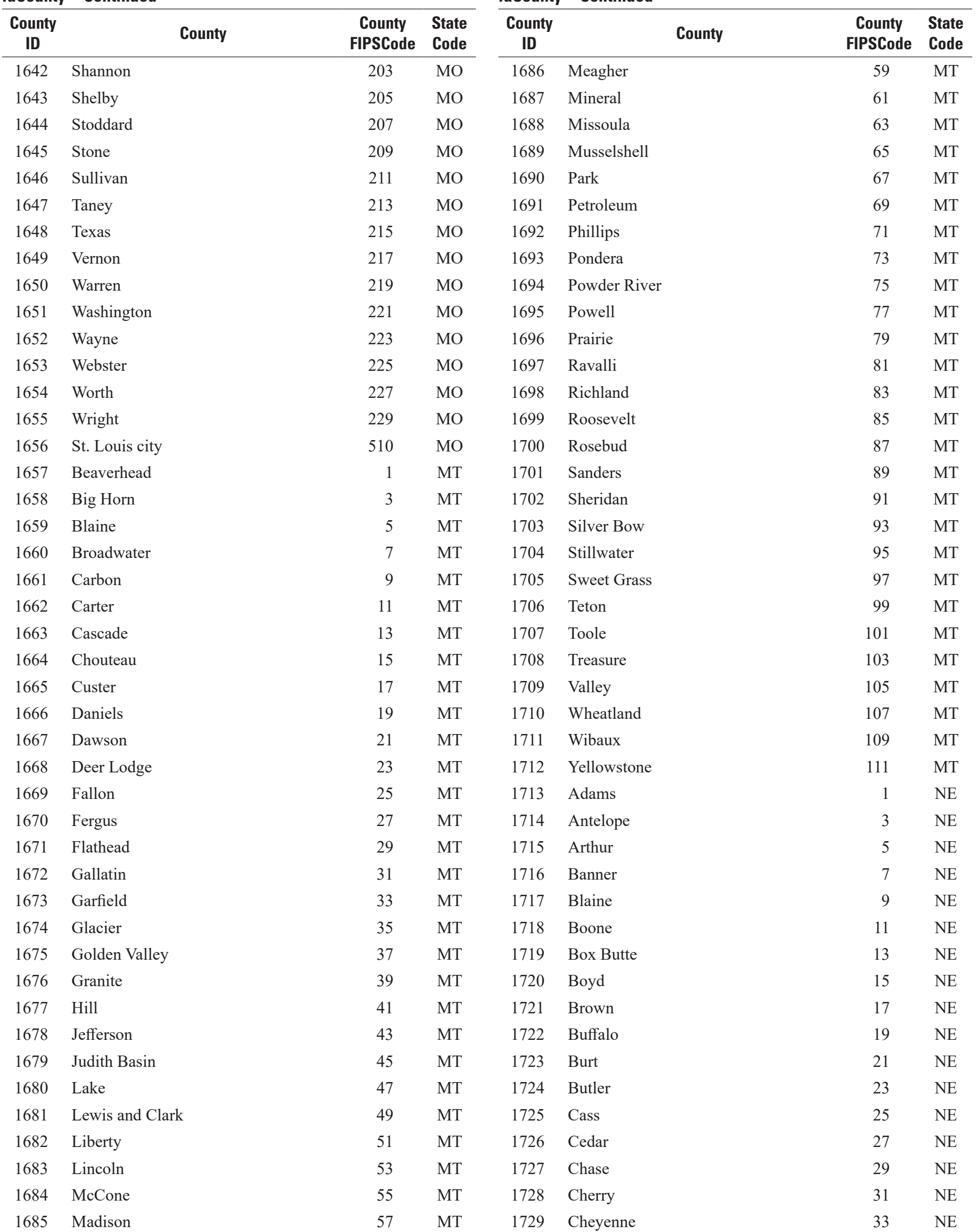


luCounty-Continued

\begin{tabular}{|c|c|c|c|c|c|c|c|}
\hline \multicolumn{4}{|c|}{ luCounty-Continued } & \multicolumn{4}{|c|}{ luCounty-Continued } \\
\hline $\begin{array}{c}\text { County } \\
\text { ID }\end{array}$ & County & $\begin{array}{l}\text { County } \\
\text { FIPSCode }\end{array}$ & $\begin{array}{l}\text { State } \\
\text { Code }\end{array}$ & $\begin{array}{c}\text { County } \\
\text { ID }\end{array}$ & County & $\begin{array}{l}\text { County } \\
\text { FIPSCode }\end{array}$ & $\begin{array}{l}\text { State } \\
\text { Code }\end{array}$ \\
\hline 1730 & Clay & 35 & $\mathrm{NE}$ & 1774 & Morrill & 123 & $\mathrm{NE}$ \\
\hline 1731 & Colfax & 37 & $\mathrm{NE}$ & 1775 & Nance & 125 & $\mathrm{NE}$ \\
\hline 1732 & Cuming & 39 & $\mathrm{NE}$ & 1776 & Nemaha & 127 & $\mathrm{NE}$ \\
\hline 1733 & Custer & 41 & $\mathrm{NE}$ & 1777 & Nuckolls & 129 & $\mathrm{NE}$ \\
\hline 1734 & Dakota & 43 & $\mathrm{NE}$ & 1778 & Otoe & 131 & $\mathrm{NE}$ \\
\hline 1735 & Dawes & 45 & $\mathrm{NE}$ & 1779 & Pawnee & 133 & $\mathrm{NE}$ \\
\hline 1736 & Dawson & 47 & $\mathrm{NE}$ & 1780 & Perkins & 135 & $\mathrm{NE}$ \\
\hline 1737 & Deuel & 49 & $\mathrm{NE}$ & 1781 & Phelps & 137 & $\mathrm{NE}$ \\
\hline 1738 & Dixon & 51 & $\mathrm{NE}$ & 1782 & Pierce & 139 & $\mathrm{NE}$ \\
\hline 1739 & Dodge & 53 & $\mathrm{NE}$ & 1783 & Platte & 141 & $\mathrm{NE}$ \\
\hline 1740 & Douglas & 55 & NE & 1784 & Polk & 143 & $\mathrm{NE}$ \\
\hline 1741 & Dundy & 57 & NE & 1785 & Red Willow & 145 & $\mathrm{NE}$ \\
\hline 1742 & Fillmore & 59 & $\mathrm{NE}$ & 1786 & Richardson & 147 & $\mathrm{NE}$ \\
\hline 1743 & Franklin & 61 & $\mathrm{NE}$ & 1787 & Rock & 149 & $\mathrm{NE}$ \\
\hline 1744 & Frontier & 63 & $\mathrm{NE}$ & 1788 & Saline & 151 & $\mathrm{NE}$ \\
\hline 1745 & Furnas & 65 & $\mathrm{NE}$ & 1789 & Sarpy & 153 & $\mathrm{NE}$ \\
\hline 1746 & Gage & 67 & NE & 1790 & Saunders & 155 & $\mathrm{NE}$ \\
\hline 1747 & Garden & 69 & $\mathrm{NE}$ & 1791 & Scotts Bluff & 157 & $\mathrm{NE}$ \\
\hline 1748 & Garfield & 71 & $\mathrm{NE}$ & 1792 & Seward & 159 & $\mathrm{NE}$ \\
\hline 1749 & Gosper & 73 & $\mathrm{NE}$ & 1793 & Sheridan & 161 & $\mathrm{NE}$ \\
\hline 1750 & Grant & 75 & $\mathrm{NE}$ & 1794 & Sherman & 163 & $\mathrm{NE}$ \\
\hline 1751 & Greeley & 77 & NE & 1795 & Sioux & 165 & $\mathrm{NE}$ \\
\hline 1752 & Hall & 79 & NE & 1796 & Stanton & 167 & $\mathrm{NE}$ \\
\hline 1753 & Hamilton & 81 & $\mathrm{NE}$ & 1797 & Thayer & 169 & $\mathrm{NE}$ \\
\hline 1754 & Harlan & 83 & $\mathrm{NE}$ & 1798 & Thomas & 171 & $\mathrm{NE}$ \\
\hline 1755 & Hayes & 85 & $\mathrm{NE}$ & 1799 & Thurston & 173 & $\mathrm{NE}$ \\
\hline 1756 & Hitchcock & 87 & $\mathrm{NE}$ & 1800 & Valley & 175 & $\mathrm{NE}$ \\
\hline 1757 & Holt & 89 & $\mathrm{NE}$ & 1801 & Washington & 177 & $\mathrm{NE}$ \\
\hline 1758 & Hooker & 91 & $\mathrm{NE}$ & 1802 & Wayne & 179 & $\mathrm{NE}$ \\
\hline 1759 & Howard & 93 & $\mathrm{NE}$ & 1803 & Webster & 181 & $\mathrm{NE}$ \\
\hline 1760 & Jefferson & 95 & $\mathrm{NE}$ & 1804 & Wheeler & 183 & $\mathrm{NE}$ \\
\hline 1761 & Johnson & 97 & $\mathrm{NE}$ & 1805 & York & 185 & $\mathrm{NE}$ \\
\hline 1762 & Kearney & 99 & $\mathrm{NE}$ & 1806 & Churchill & 1 & NV \\
\hline 1763 & Keith & 101 & NE & 1807 & Clark & 3 & NV \\
\hline 1764 & Keya Paha & 103 & $\mathrm{NE}$ & 1808 & Douglas & 5 & NV \\
\hline 1765 & Kimball & 105 & $\mathrm{NE}$ & 1809 & Elko & 7 & NV \\
\hline 1766 & Knox & 107 & $\mathrm{NE}$ & 1810 & Esmeralda & 9 & NV \\
\hline 1767 & Lancaster & 109 & NE & 1811 & Eureka & 11 & NV \\
\hline 1768 & Lincoln & 111 & NE & 1812 & Humboldt & 13 & NV \\
\hline 1769 & Logan & 113 & $\mathrm{NE}$ & 1813 & Lander & 15 & NV \\
\hline 1770 & Loup & 115 & $\mathrm{NE}$ & 1814 & Lincoln & 17 & NV \\
\hline 1771 & McPherson & 117 & $\mathrm{NE}$ & 1815 & Lyon & 19 & NV \\
\hline 1772 & Madison & 119 & $\mathrm{NE}$ & 1816 & Mineral & 21 & NV \\
\hline 1773 & Merrick & 121 & $\mathrm{NE}$ & 1817 & Nye & 23 & NV \\
\hline
\end{tabular}

luCounty-Continued 
luCounty-Continued

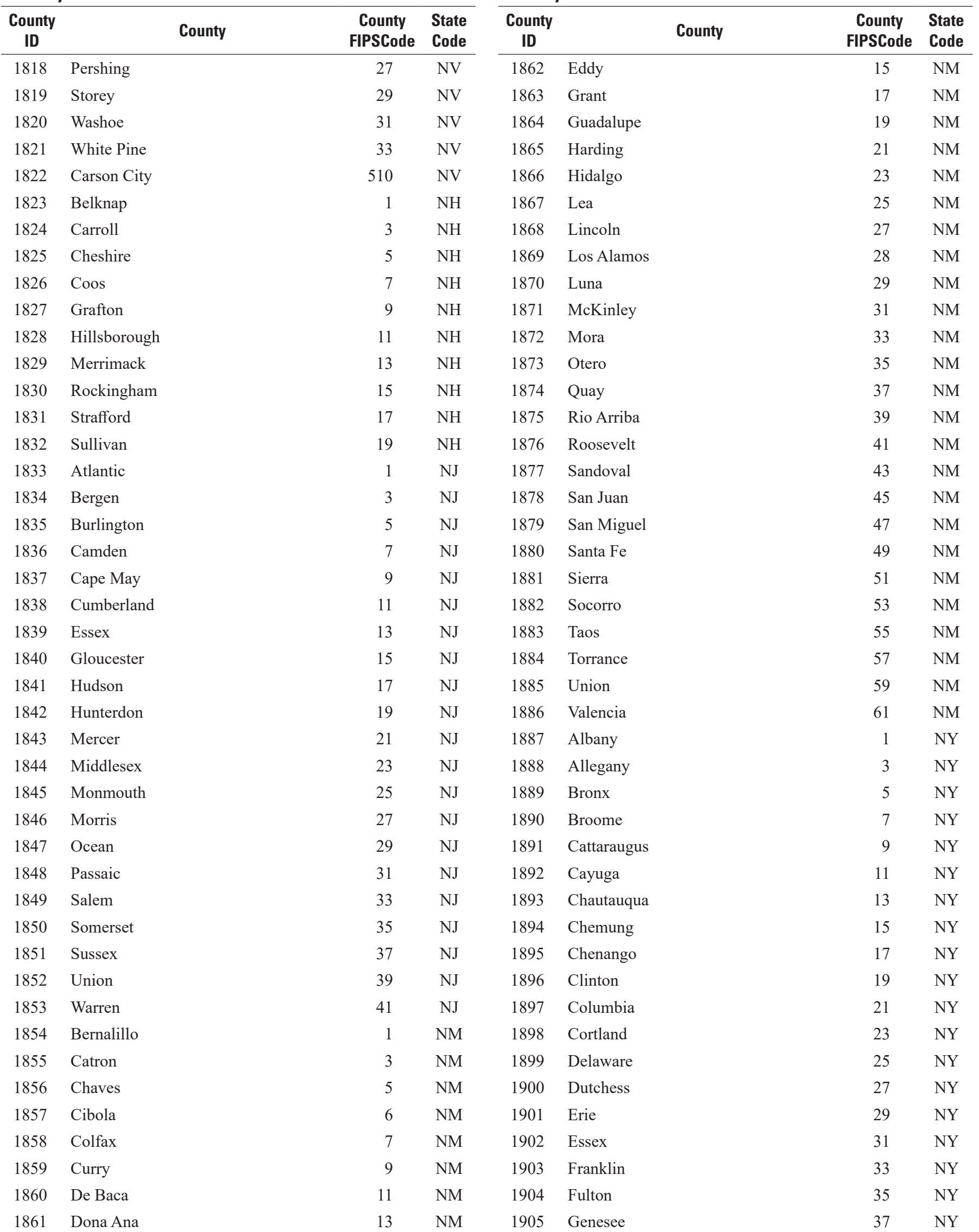




\section{luCounty-Continued}

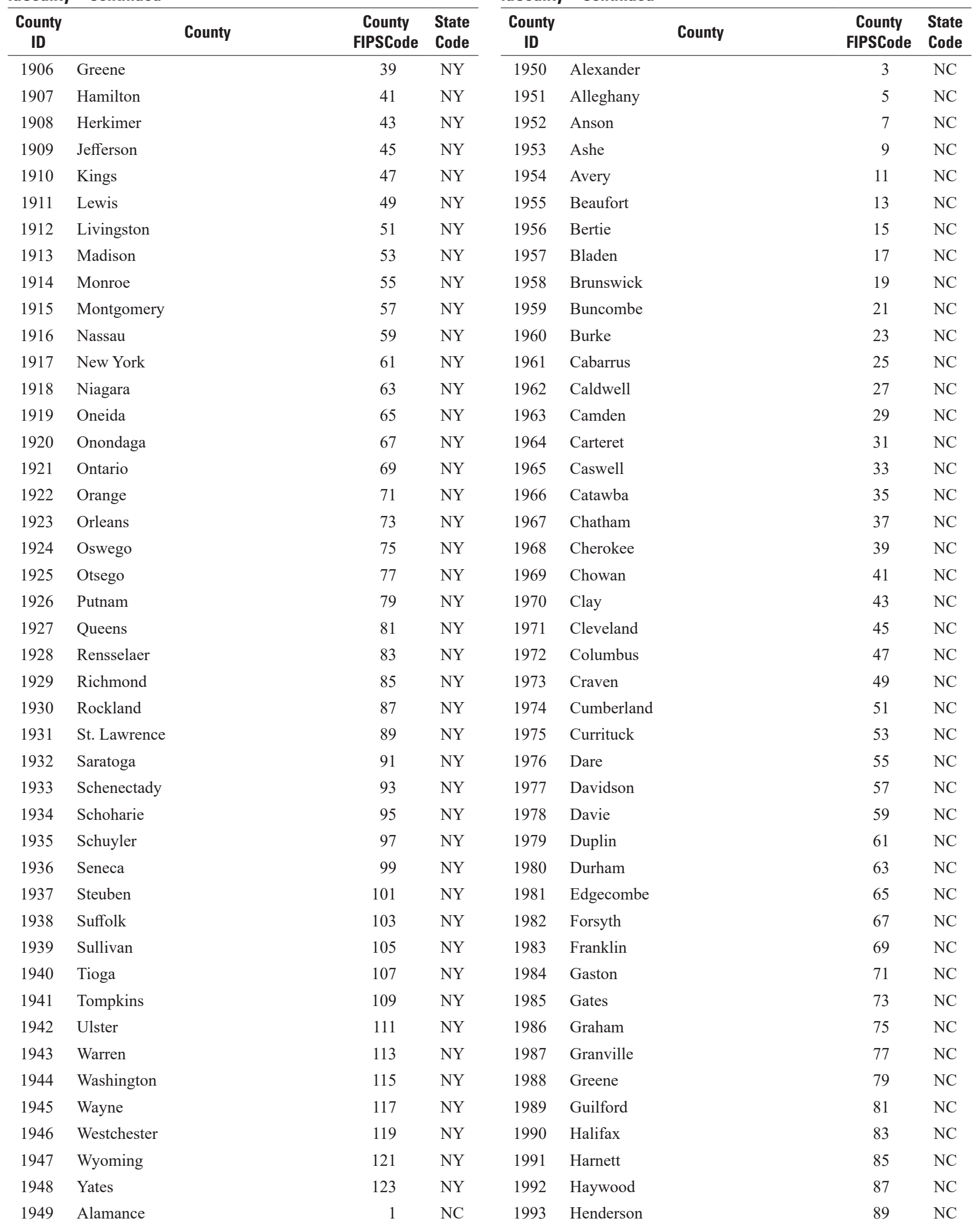


IuCounty-Continued

\begin{tabular}{|c|c|c|c|c|c|c|c|}
\hline $\begin{array}{c}\text { County } \\
\text { ID }\end{array}$ & County & $\begin{array}{l}\text { County } \\
\text { FIPSCode }\end{array}$ & $\begin{array}{l}\text { State } \\
\text { Code }\end{array}$ & $\begin{array}{c}\text { County } \\
\text { ID }\end{array}$ & County & $\begin{array}{l}\text { County } \\
\text { FIPSCode }\end{array}$ & $\begin{array}{l}\text { State } \\
\text { Code }\end{array}$ \\
\hline 1994 & Hertford & 91 & $\mathrm{NC}$ & 2038 & Union & 179 & $\mathrm{NC}$ \\
\hline 1996 & Hyde & 95 & $\mathrm{NC}$ & 2040 & Wake & 183 & $\mathrm{NC}$ \\
\hline 1997 & Iredell & 97 & $\mathrm{NC}$ & 2041 & Warren & 185 & $\mathrm{NC}$ \\
\hline 2000 & Jones & 103 & $\mathrm{NC}$ & 2044 & Wayne & 191 & $\mathrm{NC}$ \\
\hline 2001 & Lee & 105 & $\mathrm{NC}$ & 2045 & Wilkes & 193 & $\mathrm{NC}$ \\
\hline 2002 & Lenoir & 107 & $\mathrm{NC}$ & 2046 & Wilson & 195 & $\mathrm{NC}$ \\
\hline 2003 & Lincoln & 109 & $\mathrm{NC}$ & 2047 & Yadkin & 197 & $\mathrm{NC}$ \\
\hline 2007 & Martin & 117 & $\mathrm{NC}$ & 2051 & Benson & 5 & ND \\
\hline 2008 & Mecklenburg & 119 & $\mathrm{NC}$ & 2052 & Billings & 7 & ND \\
\hline 2009 & Mitchell & 121 & $\mathrm{NC}$ & 2053 & Bottineau & 9 & ND \\
\hline 2010 & Montgomery & 123 & $\mathrm{NC}$ & 2054 & Bowman & 11 & ND \\
\hline 2011 & Moore & 125 & $\mathrm{NC}$ & 2055 & Burke & 13 & ND \\
\hline 2012 & Nash & 127 & $\mathrm{NC}$ & 2056 & Burleigh & 15 & ND \\
\hline 2013 & New Hanover & 129 & $\mathrm{NC}$ & 2057 & Cass & 17 & ND \\
\hline 2014 & Northampton & 131 & $\mathrm{NC}$ & 2058 & Cavalier & 19 & ND \\
\hline 2015 & Onslow & 133 & $\mathrm{NC}$ & 2059 & Dickey & 21 & ND \\
\hline 2021 & Person & 145 & $\mathrm{NC}$ & 2065 & Golden Valley & 33 & ND \\
\hline 2022 & Pitt & 147 & $\mathrm{NC}$ & 2066 & Grand Forks & 35 & ND \\
\hline 2023 & Polk & 149 & $\mathrm{NC}$ & 2067 & Grant & 37 & ND \\
\hline 2024 & Randolph & 151 & $\mathrm{NC}$ & 2068 & Griggs & 39 & ND \\
\hline 2025 & Richmond & 153 & $\mathrm{NC}$ & 2069 & Hettinger & 41 & ND \\
\hline 2026 & Robeson & 155 & $\mathrm{NC}$ & 2070 & Kidder & 43 & ND \\
\hline 2027 & Rockingham & 157 & $\mathrm{NC}$ & 2071 & LaMoure & 45 & ND \\
\hline 2028 & Rowan & 159 & $\mathrm{NC}$ & 2072 & Logan & 47 & ND \\
\hline 2029 & Rutherford & 161 & $\mathrm{NC}$ & 2073 & McHenry & 49 & ND \\
\hline 2030 & Sampson & 163 & $\mathrm{NC}$ & 2074 & McIntosh & 51 & ND \\
\hline 2031 & Scotland & 165 & $\mathrm{NC}$ & 2075 & McKenzie & 53 & ND \\
\hline 2032 & Stanly & 167 & $\mathrm{NC}$ & 2076 & McLean & 55 & ND \\
\hline 2033 & Stokes & 169 & $\mathrm{NC}$ & 2077 & Mercer & 57 & ND \\
\hline 2034 & Surry & 171 & $\mathrm{NC}$ & 2078 & Morton & 59 & ND \\
\hline 2035 & Swain & 173 & $\mathrm{NC}$ & 2079 & Mountrail & 61 & ND \\
\hline 2036 & Transylvania & 175 & $\mathrm{NC}$ & 2080 & Nelson & 63 & ND \\
\hline 2037 & Tyrrell & 177 & $\mathrm{NC}$ & 2081 & Oliver & 65 & ND \\
\hline
\end{tabular}




\begin{tabular}{|c|c|c|c|c|c|c|c|}
\hline \multicolumn{4}{|c|}{ luCounty-Continued } & \multicolumn{4}{|c|}{ IuCounty-Continued } \\
\hline $\begin{array}{l}\text { County } \\
\text { ID }\end{array}$ & County & $\begin{array}{l}\text { County } \\
\text { FIPSCode }\end{array}$ & $\begin{array}{l}\text { State } \\
\text { Code }\end{array}$ & $\begin{array}{c}\text { County } \\
\text { ID }\end{array}$ & County & $\begin{array}{l}\text { County } \\
\text { FIPSCode }\end{array}$ & $\begin{array}{l}\text { State } \\
\text { Code }\end{array}$ \\
\hline 2083 & Pierce & 69 & ND & 2127 & Fulton & 51 & $\mathrm{OH}$ \\
\hline 2084 & Ramsey & 71 & ND & 2128 & Gallia & 53 & $\mathrm{OH}$ \\
\hline 2087 & Richland & 77 & ND & 2131 & Guernsey & 59 & $\mathrm{OH}$ \\
\hline 2088 & Rolette & 79 & ND & 2132 & Hamilton & 61 & $\mathrm{OH}$ \\
\hline 2089 & Sargent & 81 & ND & 2133 & Hancock & 63 & $\mathrm{OH}$ \\
\hline 2090 & Sheridan & 83 & ND & 2134 & Hardin & 65 & $\mathrm{OH}$ \\
\hline 2094 & Steele & 91 & ND & 2138 & Hocking & 73 & $\mathrm{OH}$ \\
\hline 2095 & Stutsman & 93 & ND & 2139 & Holmes & 75 & $\mathrm{OH}$ \\
\hline 2096 & Towner & 95 & ND & 2140 & Huron & 77 & $\mathrm{OH}$ \\
\hline 2097 & Traill & 97 & ND & 2141 & Jackson & 79 & $\mathrm{OH}$ \\
\hline 2098 & Walsh & 99 & ND & 2142 & Jefferson & 81 & $\mathrm{OH}$ \\
\hline 2099 & Ward & 101 & ND & 2143 & Knox & 83 & $\mathrm{OH}$ \\
\hline 2100 & Wells & 103 & ND & 2144 & Lake & 85 & $\mathrm{OH}$ \\
\hline 2101 & Williams & 105 & ND & 2145 & Lawrence & 87 & $\mathrm{OH}$ \\
\hline 2102 & Adams & 1 & $\mathrm{OH}$ & 2146 & Licking & 89 & $\mathrm{OH}$ \\
\hline 2109 & Brown & 15 & $\mathrm{OH}$ & 2153 & Medina & 103 & $\mathrm{OH}$ \\
\hline 2110 & Butler & 17 & $\mathrm{OH}$ & 2154 & Meigs & 105 & $\mathrm{OH}$ \\
\hline 2111 & Carroll & 19 & $\mathrm{OH}$ & 2155 & Mercer & 107 & $\mathrm{OH}$ \\
\hline 2112 & Champaign & 21 & $\mathrm{OH}$ & 2156 & Miami & 109 & $\mathrm{OH}$ \\
\hline 2113 & Clark & 23 & $\mathrm{OH}$ & 2157 & Monroe & 111 & $\mathrm{OH}$ \\
\hline 2114 & Clermont & 25 & $\mathrm{OH}$ & 2158 & Montgomery & 113 & $\mathrm{OH}$ \\
\hline 2115 & Clinton & 27 & $\mathrm{OH}$ & 2159 & Morgan & 115 & $\mathrm{OH}$ \\
\hline 2116 & Columbiana & 29 & $\mathrm{OH}$ & 2160 & Morrow & 117 & $\mathrm{OH}$ \\
\hline 2117 & Coshocton & 31 & $\mathrm{OH}$ & 2161 & Muskingum & 119 & $\mathrm{OH}$ \\
\hline 2118 & Crawford & 33 & $\mathrm{OH}$ & 2162 & Noble & 121 & $\mathrm{OH}$ \\
\hline 2119 & Cuyahoga & 35 & $\mathrm{OH}$ & 2163 & Ottawa & 123 & $\mathrm{OH}$ \\
\hline 2120 & Darke & 37 & $\mathrm{OH}$ & 2164 & Paulding & 125 & $\mathrm{OH}$ \\
\hline 2121 & Defiance & 39 & $\mathrm{OH}$ & 2165 & Perry & 127 & $\mathrm{OH}$ \\
\hline 2122 & Delaware & 41 & $\mathrm{OH}$ & 2166 & Pickaway & 129 & $\mathrm{OH}$ \\
\hline 2123 & Erie & 43 & $\mathrm{OH}$ & 2167 & Pike & 131 & $\mathrm{OH}$ \\
\hline 2124 & Fairfield & 45 & $\mathrm{OH}$ & 2168 & Portage & 133 & $\mathrm{OH}$ \\
\hline 2125 & Fayette & 47 & $\mathrm{OH}$ & 2169 & Preble & 135 & $\mathrm{OH}$ \\
\hline
\end{tabular}


luCounty-Continued

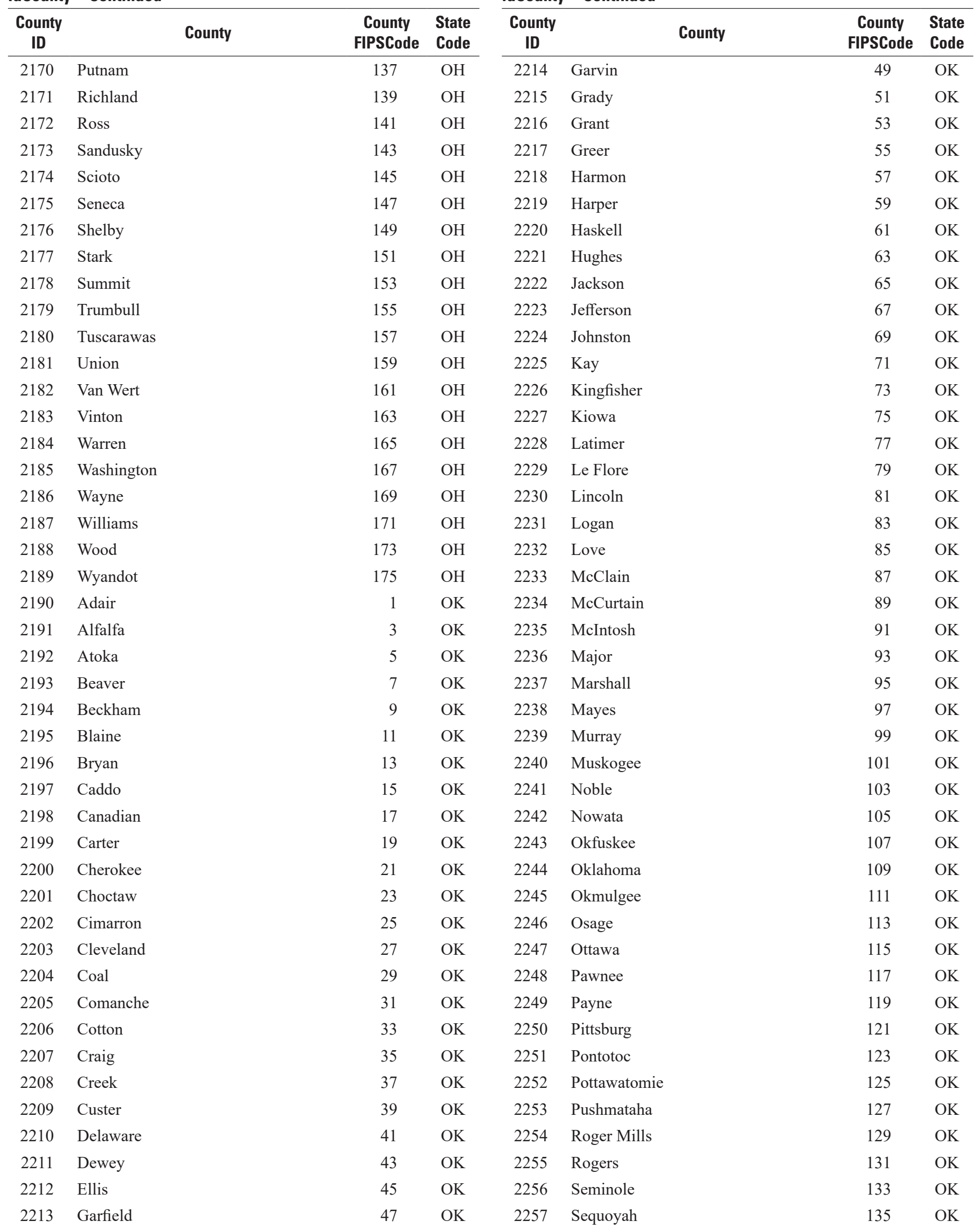




\begin{tabular}{|c|c|c|c|c|c|c|c|}
\hline \multicolumn{4}{|c|}{ luCounty-Continued } & \multicolumn{4}{|c|}{ luCounty-Continued } \\
\hline $\begin{array}{l}\text { County } \\
\text { ID }\end{array}$ & County & $\begin{array}{l}\text { County } \\
\text { FIPSCode }\end{array}$ & $\begin{array}{l}\text { State } \\
\text { Code }\end{array}$ & $\begin{array}{l}\text { County } \\
\text { ID }\end{array}$ & County & $\begin{array}{l}\text { County } \\
\text { FIPSCode }\end{array}$ & $\begin{array}{l}\text { State } \\
\text { Code }\end{array}$ \\
\hline 2258 & Stephens & 137 & $\mathrm{OK}$ & 2302 & Yamhill & 71 & OR \\
\hline 2259 & Texas & 139 & $\mathrm{OK}$ & 2303 & Adams & 1 & $\mathrm{PA}$ \\
\hline 2260 & Tillman & 141 & $\mathrm{OK}$ & 2304 & Allegheny & 3 & PA \\
\hline 2261 & Tulsa & 143 & $\mathrm{OK}$ & 2305 & Armstrong & 5 & PA \\
\hline 2262 & Wagoner & 145 & OK & 2306 & Beaver & 7 & PA \\
\hline 2263 & Washington & 147 & $\mathrm{OK}$ & 2307 & Bedford & 9 & PA \\
\hline 2264 & Washita & 149 & $\mathrm{OK}$ & 2308 & Berks & 11 & $\mathrm{PA}$ \\
\hline 2265 & Woods & 151 & $\mathrm{OK}$ & 2309 & Blair & 13 & $\mathrm{PA}$ \\
\hline 2266 & Woodward & 153 & $\mathrm{OK}$ & 2310 & Bradford & 15 & $\mathrm{PA}$ \\
\hline 2267 & Baker & 1 & OR & 2311 & Bucks & 17 & PA \\
\hline 2268 & Benton & 3 & OR & 2312 & Butler & 19 & PA \\
\hline 2269 & Clackamas & 5 & OR & 2313 & Cambria & 21 & $\mathrm{PA}$ \\
\hline 2270 & Clatsop & 7 & OR & 2314 & Cameron & 23 & $\mathrm{PA}$ \\
\hline 2271 & Columbia & 9 & OR & 2315 & Carbon & 25 & PA \\
\hline 2272 & Coos & 11 & OR & 2316 & Centre & 27 & PA \\
\hline 2273 & Crook & 13 & OR & 2317 & Chester & 29 & PA \\
\hline 2274 & Curry & 15 & OR & 2318 & Clarion & 31 & $\mathrm{PA}$ \\
\hline 2275 & Deschutes & 17 & OR & 2319 & Clearfield & 33 & $\mathrm{PA}$ \\
\hline 2276 & Douglas & 19 & OR & 2320 & Clinton & 35 & $\mathrm{PA}$ \\
\hline 2277 & Gilliam & 21 & OR & 2321 & Columbia & 37 & $\mathrm{PA}$ \\
\hline 2278 & Grant & 23 & OR & 2322 & Crawford & 39 & $\mathrm{PA}$ \\
\hline 2279 & Harney & 25 & OR & 2323 & Cumberland & 41 & $\mathrm{PA}$ \\
\hline 2280 & Hood River & 27 & OR & 2324 & Dauphin & 43 & $\mathrm{PA}$ \\
\hline 2281 & Jackson & 29 & OR & 2325 & Delaware & 45 & $\mathrm{PA}$ \\
\hline 2282 & Jefferson & 31 & OR & 2326 & Elk & 47 & $\mathrm{PA}$ \\
\hline 2283 & Josephine & 33 & OR & 2327 & Erie & 49 & PA \\
\hline 2284 & Klamath & 35 & OR & 2328 & Fayette & 51 & $\mathrm{PA}$ \\
\hline 2285 & Lake & 37 & OR & 2329 & Forest & 53 & $\mathrm{PA}$ \\
\hline 2286 & Lane & 39 & OR & 2330 & Franklin & 55 & $\mathrm{PA}$ \\
\hline 2287 & Lincoln & 41 & OR & 2331 & Fulton & 57 & $\mathrm{PA}$ \\
\hline 2288 & Linn & 43 & OR & 2332 & Greene & 59 & $\mathrm{PA}$ \\
\hline 2289 & Malheur & 45 & OR & 2333 & Huntingdon & 61 & $\mathrm{PA}$ \\
\hline 2290 & Marion & 47 & OR & 2334 & Indiana & 63 & $\mathrm{PA}$ \\
\hline 2291 & Morrow & 49 & OR & 2335 & Jefferson & 65 & $\mathrm{PA}$ \\
\hline 2292 & Multnomah & 51 & OR & 2336 & Juniata & 67 & PA \\
\hline 2293 & Polk & 53 & OR & 2337 & Lackawanna & 69 & $\mathrm{PA}$ \\
\hline 2294 & Sherman & 55 & OR & 2338 & Lancaster & 71 & $\mathrm{PA}$ \\
\hline 2295 & Tillamook & 57 & OR & 2339 & Lawrence & 73 & $\mathrm{PA}$ \\
\hline 2296 & Umatilla & 59 & OR & 2340 & Lebanon & 75 & $\mathrm{PA}$ \\
\hline 2297 & Union & 61 & OR & 2341 & Lehigh & 77 & $\mathrm{PA}$ \\
\hline 2298 & Wallowa & 63 & OR & 2342 & Luzerne & 79 & $\mathrm{PA}$ \\
\hline 2299 & Wasco & 65 & OR & 2343 & Lycoming & 81 & $\mathrm{PA}$ \\
\hline 2300 & Washington & 67 & OR & 2344 & McKean & 83 & $\mathrm{PA}$ \\
\hline 2301 & Wheeler & 69 & OR & 2345 & Mercer & 85 & PA \\
\hline
\end{tabular}


luCounty-Continued

\begin{tabular}{|c|c|c|c|c|c|c|c|}
\hline $\begin{array}{c}\text { County } \\
\text { ID }\end{array}$ & County & $\begin{array}{l}\text { County } \\
\text { FIPSCode }\end{array}$ & $\begin{array}{l}\text { State } \\
\text { Code }\end{array}$ & $\begin{array}{c}\text { County } \\
\text { ID }\end{array}$ & County & $\begin{array}{l}\text { County } \\
\text { FIPSCode }\end{array}$ & $\begin{array}{l}\text { State } \\
\text { Code }\end{array}$ \\
\hline 2346 & Mifflin & 87 & $\mathrm{PA}$ & 2390 & Darlington & 31 & $\mathrm{SC}$ \\
\hline 2348 & Montgomery & 91 & $\mathrm{PA}$ & 2392 & Dorchester & 35 & $\mathrm{SC}$ \\
\hline 2349 & Montour & 93 & $\mathrm{PA}$ & 2393 & Edgefield & 37 & $\mathrm{SC}$ \\
\hline 2352 & Perry & 99 & $\mathrm{PA}$ & 2396 & Georgetown & 43 & $\mathrm{SC}$ \\
\hline 2353 & Philadelphia & 101 & $\mathrm{PA}$ & 2397 & Greenville & 45 & $\mathrm{SC}$ \\
\hline 2354 & Pike & 103 & PA & 2398 & Greenwood & 47 & $\mathrm{SC}$ \\
\hline 2355 & Potter & 105 & PA & 2399 & Hampton & 49 & $\mathrm{SC}$ \\
\hline 2359 & Sullivan & 113 & PA & 2403 & Lancaster & 57 & $\mathrm{SC}$ \\
\hline 2360 & Susquehanna & 115 & PA & 2404 & Laurens & 59 & $\mathrm{SC}$ \\
\hline 2361 & Tioga & 117 & PA & 2405 & Lee & 61 & $\mathrm{SC}$ \\
\hline 2362 & Union & 119 & PA & 2406 & Lexington & 63 & $\mathrm{SC}$ \\
\hline 2363 & Venango & 121 & $\mathrm{PA}$ & 2407 & McCormick & 65 & $\mathrm{SC}$ \\
\hline 2364 & Warren & 123 & $\mathrm{PA}$ & 2408 & Marion & 67 & $\mathrm{SC}$ \\
\hline 2365 & Washington & 125 & PA & 2409 & Marlboro & 69 & $\mathrm{SC}$ \\
\hline 2366 & Wayne & 127 & $\mathrm{PA}$ & 2410 & Newberry & 71 & $\mathrm{SC}$ \\
\hline 2367 & Westmoreland & 129 & PA & 2411 & Oconee & 73 & $\mathrm{SC}$ \\
\hline 2373 & Providence & 7 & RI & 2417 & Sumter & 85 & $\mathrm{SC}$ \\
\hline 2374 & Washington & 9 & RI & 2418 & Union & 87 & $\mathrm{SC}$ \\
\hline 2375 & Abbeville & 1 & $\mathrm{SC}$ & 2419 & Williamsburg & 89 & $\mathrm{SC}$ \\
\hline 2376 & Aiken & 3 & $\mathrm{SC}$ & 2420 & York & 91 & $\mathrm{SC}$ \\
\hline 2377 & Allendale & 5 & $\mathrm{SC}$ & 2421 & Aurora & 3 & SD \\
\hline 2378 & Anderson & 7 & $\mathrm{SC}$ & 2422 & Beadle & 5 & SD \\
\hline 2379 & Bamberg & 9 & $\mathrm{SC}$ & 2423 & Bennett & 7 & SD \\
\hline 2380 & Barnwell & 11 & $\mathrm{SC}$ & 2424 & Bon Homme & 9 & SD \\
\hline 2381 & Beaufort & 13 & $\mathrm{SC}$ & 2425 & Brookings & 11 & SD \\
\hline 2382 & Berkeley & 15 & $\mathrm{SC}$ & 2426 & Brown & 13 & SD \\
\hline 2383 & Calhoun & 17 & $\mathrm{SC}$ & 2427 & Brule & 15 & $\mathrm{SD}$ \\
\hline 2384 & Charleston & 19 & $\mathrm{SC}$ & 2428 & Buffalo & 17 & SD \\
\hline 2385 & Cherokee & 21 & $\mathrm{SC}$ & 2429 & Butte & 19 & SD \\
\hline 2386 & Chester & 23 & $\mathrm{SC}$ & 2430 & Campbell & 21 & SD \\
\hline 2387 & Chesterfield & 25 & $\mathrm{SC}$ & 2431 & Charles Mix & 23 & SD \\
\hline 2388 & Clarendon & 27 & $\mathrm{SC}$ & 2432 & Clark & 25 & SD \\
\hline 2389 & Colleton & 29 & $\mathrm{SC}$ & 2433 & Clay & 27 & SD \\
\hline
\end{tabular}




\begin{tabular}{|c|c|c|c|c|c|c|c|}
\hline \multicolumn{4}{|c|}{ IuCounty-Continued } & \multicolumn{4}{|c|}{ IuCounty-Continued } \\
\hline $\begin{array}{c}\text { County } \\
\text { ID }\end{array}$ & County & $\begin{array}{l}\text { County } \\
\text { FIPSCode }\end{array}$ & $\begin{array}{l}\text { State } \\
\text { Code }\end{array}$ & $\begin{array}{c}\text { County } \\
\text { ID }\end{array}$ & County & $\begin{array}{l}\text { County } \\
\text { FIPSCode }\end{array}$ & $\begin{array}{l}\text { State } \\
\text { Code }\end{array}$ \\
\hline 2435 & Corson & 31 & SD & 2479 & Sully & 119 & SD \\
\hline 2436 & Custer & 33 & SD & 2480 & Todd & 121 & $\mathrm{SD}$ \\
\hline 2439 & Deuel & 39 & SD & 2483 & Union & 127 & $\mathrm{SD}$ \\
\hline 2440 & Dewey & 41 & SD & 2484 & Walworth & 129 & SD \\
\hline 2441 & Douglas & 43 & SD & 2485 & Yankton & 135 & $\mathrm{SD}$ \\
\hline 2442 & Edmunds & 45 & SD & 2486 & Ziebach & 137 & SD \\
\hline 2446 & Gregory & 53 & $\mathrm{SD}$ & 2490 & Bledsoe & 7 & $\mathrm{TN}$ \\
\hline 2447 & Haakon & 55 & SD & 2491 & Blount & 9 & $\mathrm{TN}$ \\
\hline 2448 & Hamlin & 57 & SD & 2492 & Bradley & 11 & $\mathrm{TN}$ \\
\hline 2449 & Hand & 59 & SD & 2493 & Campbell & 13 & $\mathrm{TN}$ \\
\hline 2450 & Hanson & 61 & SD & 2494 & Cannon & 15 & $\mathrm{TN}$ \\
\hline 2451 & Harding & 63 & SD & 2495 & Carroll & 17 & $\mathrm{TN}$ \\
\hline 2452 & Hughes & 65 & SD & 2496 & Carter & 19 & $\mathrm{TN}$ \\
\hline 2453 & Hutchinson & 67 & $\mathrm{SD}$ & 2497 & Cheatham & 21 & $\mathrm{TN}$ \\
\hline 2454 & Hyde & 69 & SD & 2498 & Chester & 23 & $\mathrm{TN}$ \\
\hline 2461 & Lincoln & 83 & SD & 2505 & Davidson & 37 & $\mathrm{TN}$ \\
\hline 2462 & Lyman & 85 & SD & 2506 & Decatur & 39 & $\mathrm{TN}$ \\
\hline 2463 & McCook & 87 & $\mathrm{SD}$ & 2507 & DeKalb & 41 & $\mathrm{TN}$ \\
\hline 2464 & McPherson & 89 & SD & 2508 & Dickson & 43 & $\mathrm{TN}$ \\
\hline 2465 & Marshall & 91 & SD & 2509 & Dyer & 45 & $\mathrm{TN}$ \\
\hline 2466 & Meade & 93 & SD & 2510 & Fayette & 47 & $\mathrm{TN}$ \\
\hline 2467 & Mellette & 95 & SD & 2511 & Fentress & 49 & $\mathrm{TN}$ \\
\hline 2468 & Miner & 97 & SD & 2512 & Franklin & 51 & $\mathrm{TN}$ \\
\hline 2469 & Minnehaha & 99 & $\mathrm{SD}$ & 2513 & Gibson & 53 & $\mathrm{TN}$ \\
\hline 2470 & Moody & 101 & SD & 2514 & Giles & 55 & $\mathrm{TN}$ \\
\hline 2471 & Pennington & 103 & $\mathrm{SD}$ & 2515 & Grainger & 57 & $\mathrm{TN}$ \\
\hline 2472 & Perkins & 105 & SD & 2516 & Greene & 59 & $\mathrm{TN}$ \\
\hline 2473 & Potter & 107 & SD & 2517 & Grundy & 61 & $\mathrm{TN}$ \\
\hline 2474 & Roberts & 109 & SD & 2518 & Hamblen & 63 & $\mathrm{TN}$ \\
\hline 2475 & Sanborn & 111 & $\mathrm{SD}$ & 2519 & Hamilton & 65 & $\mathrm{TN}$ \\
\hline 2476 & Shannon & 113 & $\mathrm{SD}$ & 2520 & Hancock & 67 & $\mathrm{TN}$ \\
\hline 2477 & Spink & 115 & SD & 2521 & Hardeman & 69 & $\mathrm{TN}$ \\
\hline
\end{tabular}


luCounty-Continued

\begin{tabular}{|c|c|c|c|c|c|c|c|}
\hline $\begin{array}{c}\text { County } \\
\text { ID }\end{array}$ & County & $\begin{array}{l}\text { County } \\
\text { FIPSCode }\end{array}$ & $\begin{array}{l}\text { State } \\
\text { Code }\end{array}$ & $\begin{array}{c}\text { County } \\
\text { ID }\end{array}$ & County & $\begin{array}{l}\text { County } \\
\text { FIPSCode }\end{array}$ & $\begin{array}{l}\text { State } \\
\text { Code }\end{array}$ \\
\hline 2522 & Hardin & 71 & $\mathrm{TN}$ & 2566 & Smith & 159 & $\mathrm{TN}$ \\
\hline 2524 & Haywood & 75 & $\mathrm{TN}$ & 2568 & Sullivan & 163 & $\mathrm{TN}$ \\
\hline 2525 & Henderson & 77 & $\mathrm{TN}$ & 2569 & Sumner & 165 & $\mathrm{TN}$ \\
\hline 2528 & Houston & 83 & $\mathrm{TN}$ & 2572 & Unicoi & 171 & $\mathrm{TN}$ \\
\hline 2529 & Humphreys & 85 & $\mathrm{TN}$ & 2573 & Union & 173 & $\mathrm{TN}$ \\
\hline 2530 & Jackson & 87 & $\mathrm{TN}$ & 2574 & Van Buren & 175 & $\mathrm{TN}$ \\
\hline 2531 & Jefferson & 89 & $\mathrm{TN}$ & 2575 & Warren & 177 & $\mathrm{TN}$ \\
\hline 2535 & Lauderdale & 97 & $\mathrm{TN}$ & 2579 & White & 185 & $\mathrm{TN}$ \\
\hline 2536 & Lawrence & 99 & $\mathrm{TN}$ & 2580 & Williamson & 187 & $\mathrm{TN}$ \\
\hline 2537 & Lewis & 101 & $\mathrm{TN}$ & 2581 & Wilson & 189 & $\mathrm{TN}$ \\
\hline 2538 & Lincoln & 103 & $\mathrm{TN}$ & 2582 & Anderson & 1 & $\mathrm{TX}$ \\
\hline 2539 & Loudon & 105 & $\mathrm{TN}$ & 2583 & Andrews & 3 & TX \\
\hline 2540 & McMinn & 107 & $\mathrm{TN}$ & 2584 & Angelina & 5 & TX \\
\hline 2541 & McNairy & 109 & $\mathrm{TN}$ & 2585 & Aransas & 7 & $\mathrm{TX}$ \\
\hline 2542 & Macon & 111 & $\mathrm{TN}$ & 2586 & Archer & 9 & $\mathrm{TX}$ \\
\hline 2543 & Madison & 113 & $\mathrm{TN}$ & 2587 & Armstrong & 11 & $\mathrm{TX}$ \\
\hline 2549 & Montgomery & 125 & $\mathrm{TN}$ & 2593 & Baylor & 23 & $\mathrm{TX}$ \\
\hline 2550 & Moore & 127 & $\mathrm{TN}$ & 2594 & Bee & 25 & $\mathrm{TX}$ \\
\hline 2551 & Morgan & 129 & $\mathrm{TN}$ & 2595 & Bell & 27 & TX \\
\hline 2552 & Obion & 131 & $\mathrm{TN}$ & 2596 & Bexar & 29 & $\mathrm{TX}$ \\
\hline 2553 & Overton & 133 & $\mathrm{TN}$ & 2597 & Blanco & 31 & $\mathrm{TX}$ \\
\hline 2554 & Perry & 135 & $\mathrm{TN}$ & 2598 & Borden & 33 & $\mathrm{TX}$ \\
\hline 2555 & Pickett & 137 & $\mathrm{TN}$ & 2599 & Bosque & 35 & $\mathrm{TX}$ \\
\hline 2556 & Polk & 139 & $\mathrm{TN}$ & 2600 & Bowie & 37 & TX \\
\hline 2557 & Putnam & 141 & $\mathrm{TN}$ & 2601 & Brazoria & 39 & TX \\
\hline 2558 & Rhea & 143 & $\mathrm{TN}$ & 2602 & Brazos & 41 & $\mathrm{TX}$ \\
\hline 2559 & Roane & 145 & $\mathrm{TN}$ & 2603 & Brewster & 43 & $\mathrm{TX}$ \\
\hline 2560 & Robertson & 147 & $\mathrm{TN}$ & 2604 & Briscoe & 45 & $\mathrm{TX}$ \\
\hline 2561 & Rutherford & 149 & $\mathrm{TN}$ & 2605 & Brooks & 47 & $\mathrm{TX}$ \\
\hline 2562 & Scott & 151 & $\mathrm{TN}$ & 2606 & Brown & 49 & TX \\
\hline 2563 & Sequatchie & 153 & $\mathrm{TN}$ & 2607 & Burleson & 51 & TX \\
\hline 2564 & Sevier & 155 & $\mathrm{TN}$ & 2608 & Burnet & 53 & $\mathrm{TX}$ \\
\hline 2565 & Shelby & 157 & $\mathrm{TN}$ & 2609 & Caldwell & 55 & $\mathrm{TX}$ \\
\hline
\end{tabular}


luCounty-Continued

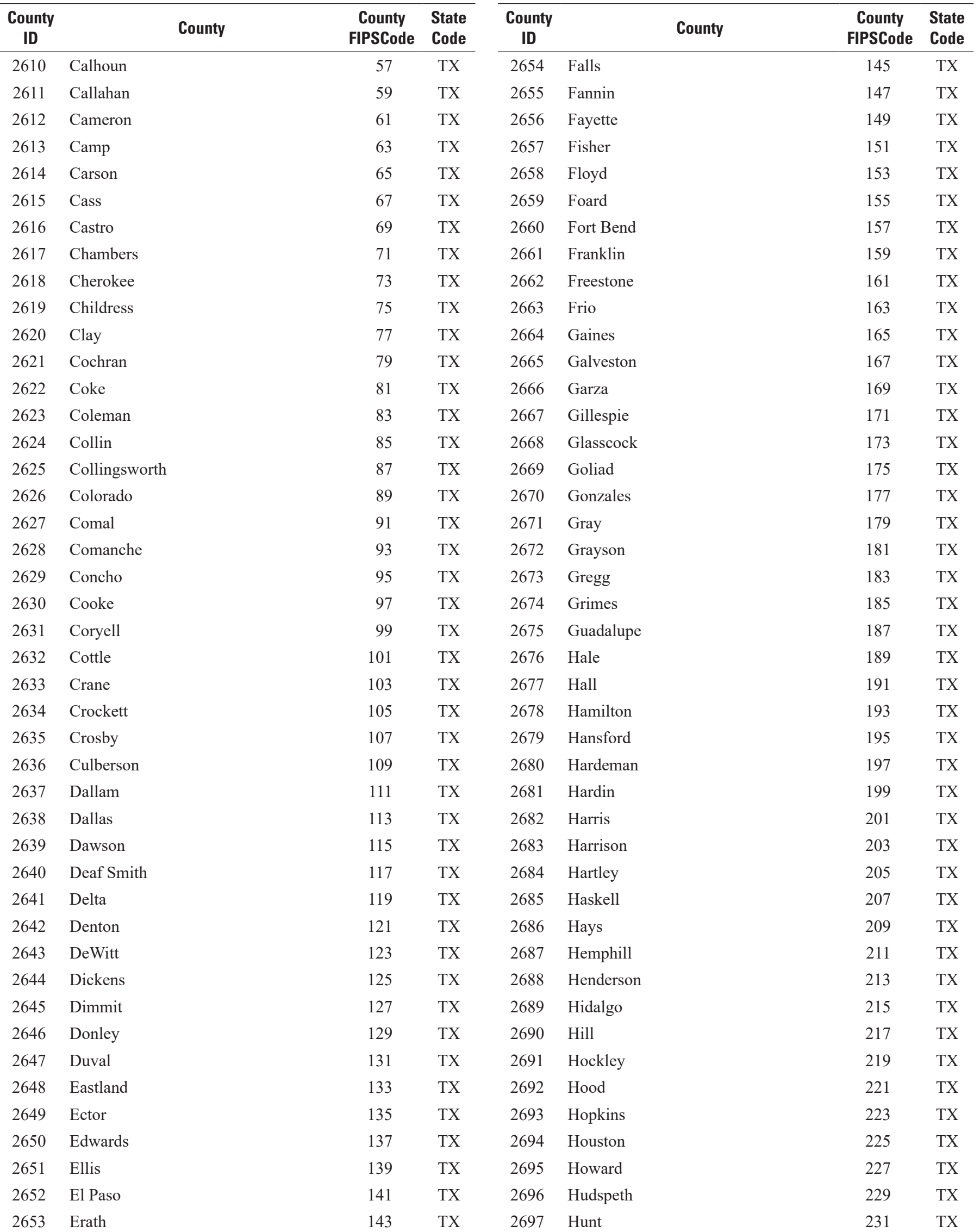


luCounty-Continued

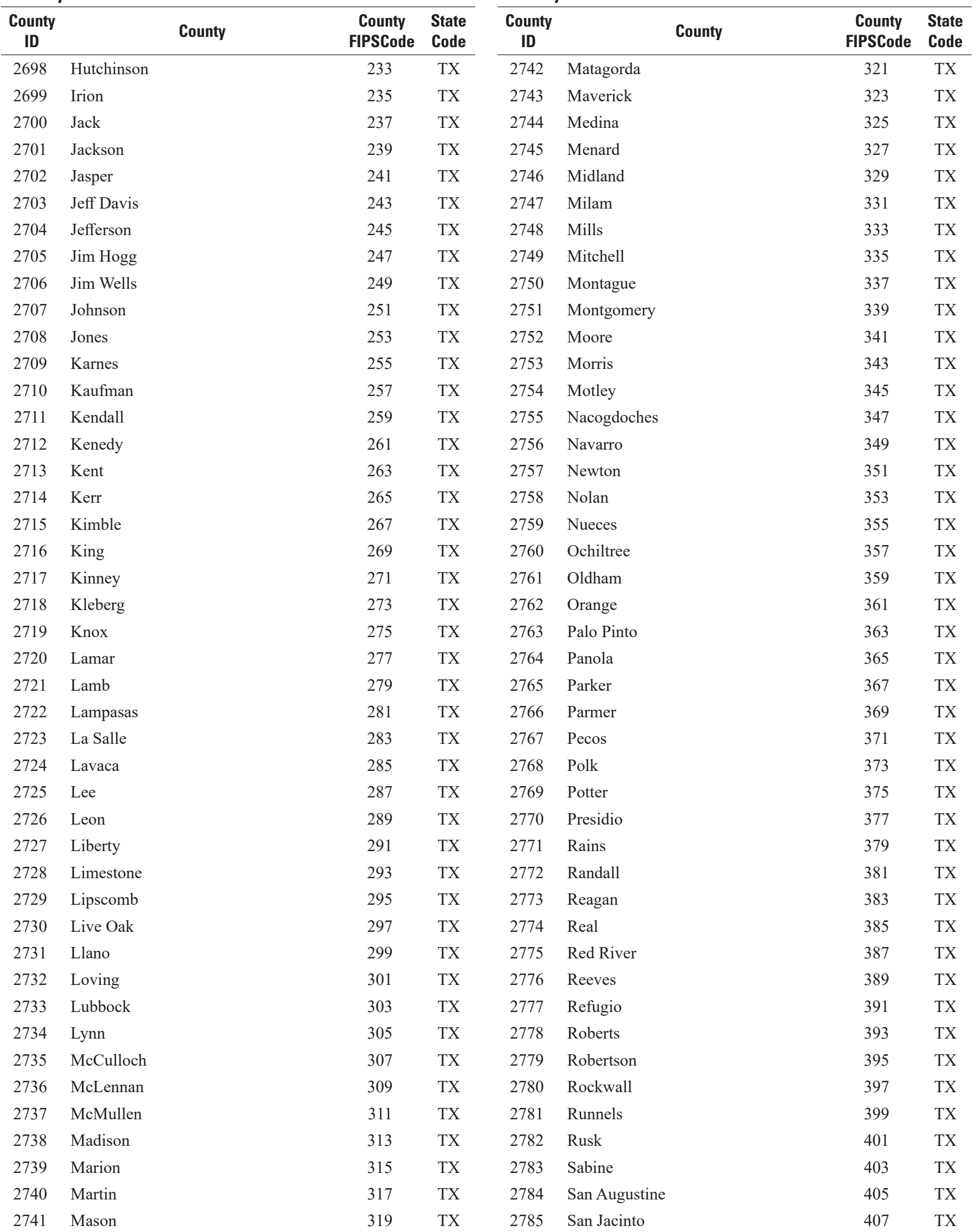




\begin{tabular}{|c|c|c|c|c|c|c|c|}
\hline \multicolumn{4}{|c|}{ IuCounty-Continued } & \multicolumn{4}{|c|}{ IuCounty-Continued } \\
\hline $\begin{array}{c}\text { County } \\
\text { ID }\end{array}$ & County & $\begin{array}{l}\text { County } \\
\text { FIPSCode }\end{array}$ & $\begin{array}{l}\text { State } \\
\text { Code }\end{array}$ & $\begin{array}{c}\text { County } \\
\text { ID }\end{array}$ & County & $\begin{array}{l}\text { County } \\
\text { FIPSCode }\end{array}$ & $\begin{array}{l}\text { State } \\
\text { Code }\end{array}$ \\
\hline 2787 & San Saba & 411 & $\mathrm{TX}$ & 2831 & Wood & 499 & $\mathrm{TX}$ \\
\hline 2788 & Schleicher & 413 & $\mathrm{TX}$ & 2832 & Yoakum & 501 & $\mathrm{TX}$ \\
\hline 2791 & Shelby & 419 & $\mathrm{TX}$ & 2835 & Zavala & 507 & $\mathrm{TX}$ \\
\hline 2792 & Sherman & 421 & $\mathrm{TX}$ & 2836 & Beaver & 1 & UT \\
\hline 2793 & Smith & 423 & $\mathrm{TX}$ & 2837 & Box Elder & 3 & UT \\
\hline 2794 & Somervell & 425 & $\mathrm{TX}$ & 2838 & Cache & 5 & UT \\
\hline 2798 & Stonewall & 433 & $\mathrm{TX}$ & 2842 & Duchesne & 13 & UT \\
\hline 2799 & Sutton & 435 & $\mathrm{TX}$ & 2843 & Emery & 15 & UT \\
\hline 2800 & Swisher & 437 & $\mathrm{TX}$ & 2844 & Garfield & 17 & UT \\
\hline 2801 & Tarrant & 439 & $\mathrm{TX}$ & 2845 & Grand & 19 & UT \\
\hline 2802 & Taylor & 441 & $\mathrm{TX}$ & 2846 & Iron & 21 & UT \\
\hline 2803 & Terrell & 443 & $\mathrm{TX}$ & 2847 & Juab & 23 & UT \\
\hline 2804 & Terry & 445 & $\mathrm{TX}$ & 2848 & Kane & 25 & UT \\
\hline 2805 & Throckmorton & 447 & $\mathrm{TX}$ & 2849 & Millard & 27 & UT \\
\hline 2806 & Titus & 449 & $\mathrm{TX}$ & 2850 & Morgan & 29 & UT \\
\hline 2813 & Uvalde & 463 & $\mathrm{TX}$ & 2857 & Summit & 43 & UT \\
\hline 2814 & Val Verde & 465 & $\mathrm{TX}$ & 2858 & Tooele & 45 & UT \\
\hline 2815 & Van Zandt & 467 & $\mathrm{TX}$ & 2859 & Uintah & 47 & UT \\
\hline 2816 & Victoria & 469 & $\mathrm{TX}$ & 2860 & Utah & 49 & UT \\
\hline 2817 & Walker & 471 & $\mathrm{TX}$ & 2861 & Wasatch & 51 & UT \\
\hline 2818 & Waller & 473 & $\mathrm{TX}$ & 2862 & Washington & 53 & UT \\
\hline 2819 & Ward & 475 & $\mathrm{TX}$ & 2863 & Wayne & 55 & UT \\
\hline 2820 & Washington & 477 & $\mathrm{TX}$ & 2864 & Weber & 57 & UT \\
\hline 2821 & Webb & 479 & $\mathrm{TX}$ & 2865 & Addison & 1 & VT \\
\hline 2822 & Wharton & 481 & $\mathrm{TX}$ & 2866 & Bennington & 3 & VT \\
\hline 2823 & Wheeler & 483 & $\mathrm{TX}$ & 2867 & Caledonia & 5 & VT \\
\hline 2824 & Wichita & 485 & $\mathrm{TX}$ & 2868 & Chittenden & 7 & VT \\
\hline 2825 & Wilbarger & 487 & $\mathrm{TX}$ & 2869 & Essex & 9 & VT \\
\hline 2826 & Willacy & 489 & $\mathrm{TX}$ & 2870 & Franklin & 11 & VT \\
\hline 2827 & Williamson & 491 & $\mathrm{TX}$ & 2871 & Grand Isle & 13 & VT \\
\hline 2828 & Wilson & 493 & $\mathrm{TX}$ & 2872 & Lamoille & 15 & VT \\
\hline 2829 & Winkler & 495 & $\mathrm{TX}$ & 2873 & Orange & 17 & VT \\
\hline
\end{tabular}


luCounty-Continued

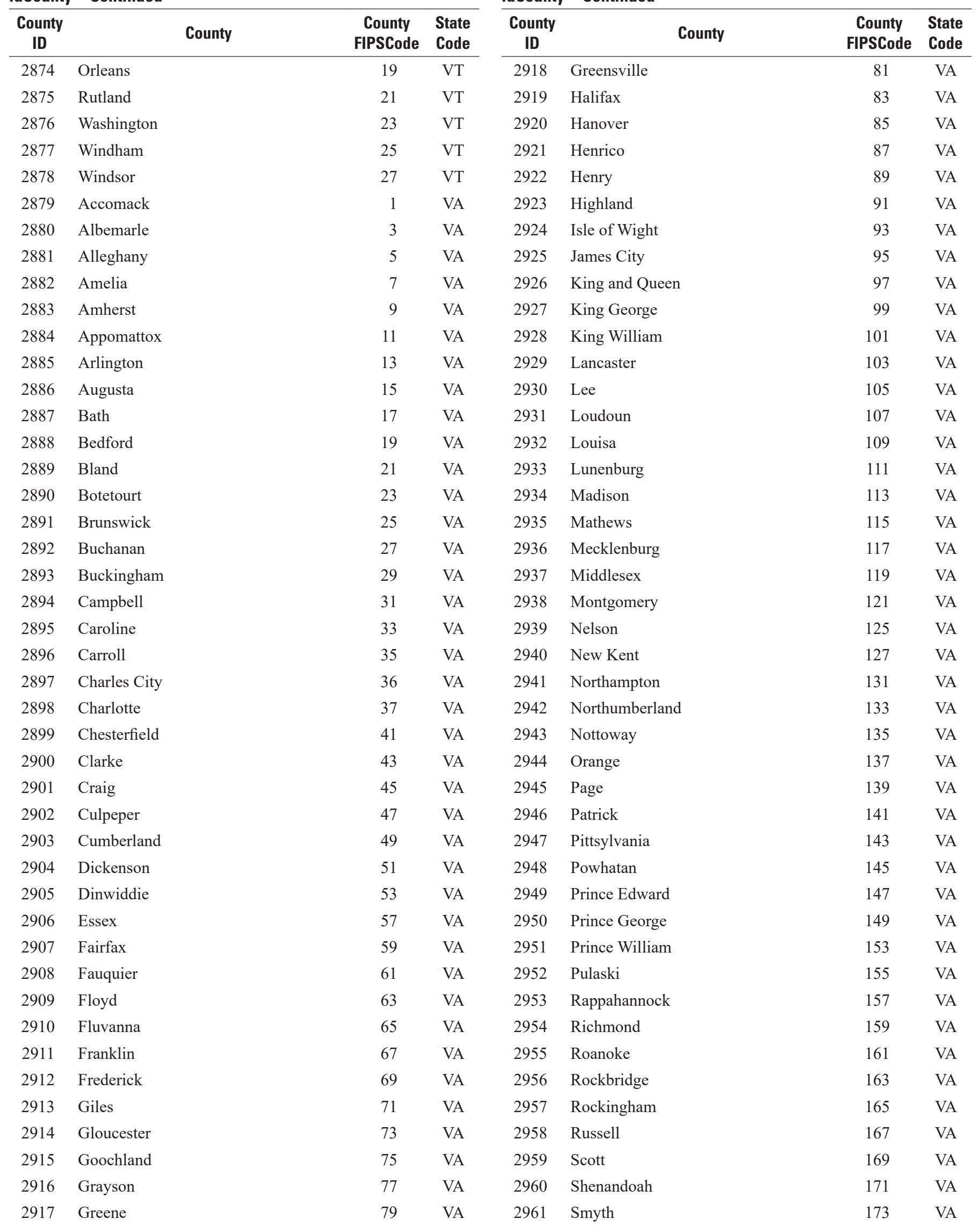




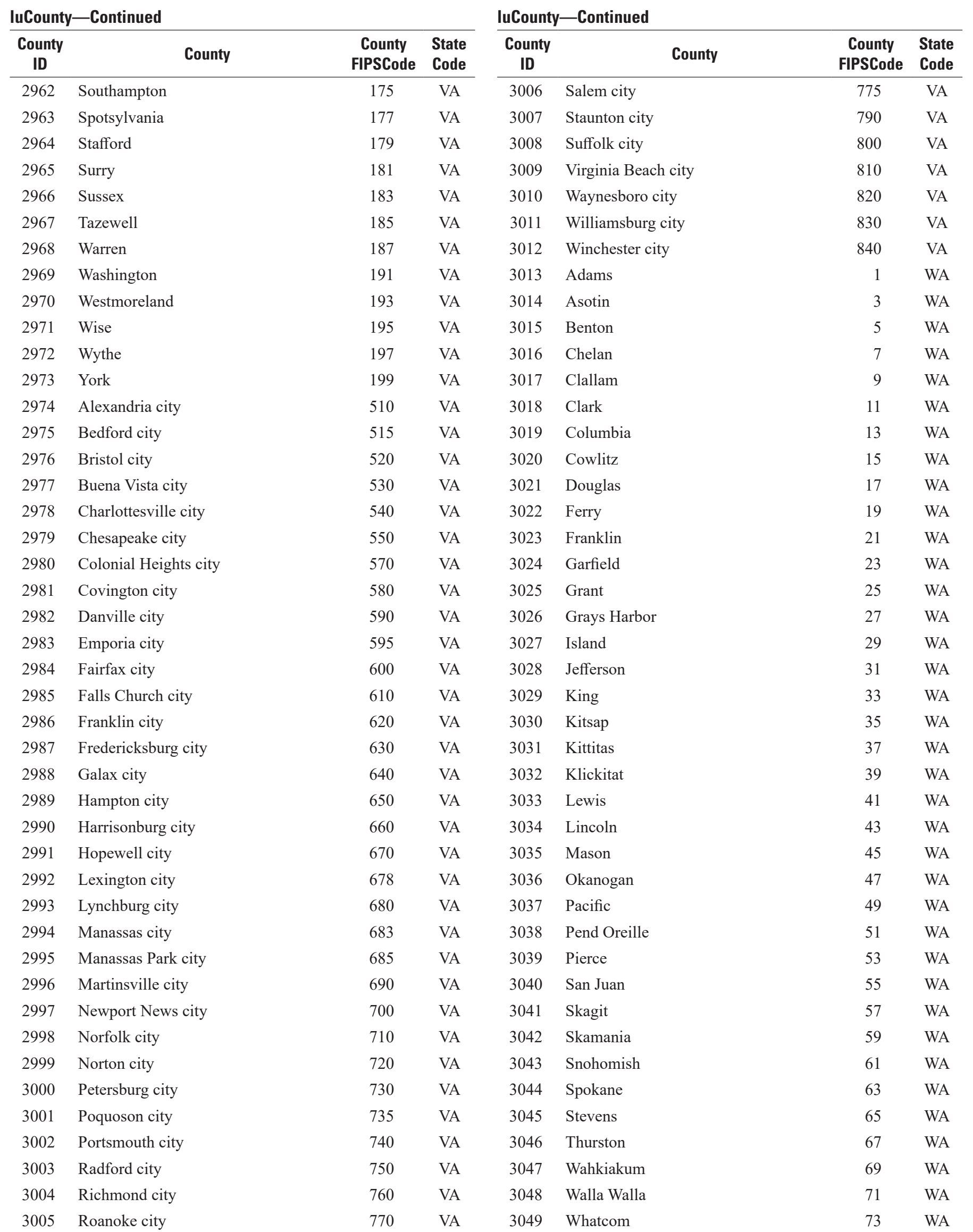


IuCounty-Continued

\begin{tabular}{|c|c|c|c|c|c|c|c|}
\hline $\begin{array}{l}\text { County } \\
\text { ID }\end{array}$ & County & $\begin{array}{l}\text { County } \\
\text { FIPSCode }\end{array}$ & $\begin{array}{l}\text { State } \\
\text { Code }\end{array}$ & $\begin{array}{l}\text { County } \\
\text { ID }\end{array}$ & County & $\begin{array}{l}\text { County } \\
\text { FIPSCode }\end{array}$ & $\begin{array}{l}\text { State } \\
\text { Code }\end{array}$ \\
\hline 3050 & Whitman & 75 & WA & 3094 & Ritchie & 85 & WV \\
\hline 3052 & Barbour & 1 & WV & 3096 & Summers & 89 & WV \\
\hline 3053 & Berkeley & 3 & WV & 3097 & Taylor & 91 & WV \\
\hline 3056 & Brooke & 9 & WV & 3100 & Upshur & 97 & WV \\
\hline 3057 & Cabell & 11 & WV & 3101 & Wayne & 99 & WV \\
\hline 3058 & Calhoun & 13 & WV & 3102 & Webster & 101 & WV \\
\hline 3059 & Clay & 15 & WV & 3103 & Wetzel & 103 & WV \\
\hline 3063 & Grant & 23 & WV & 3107 & Adams & 1 & WI \\
\hline 3064 & Greenbrier & 25 & WV & 3108 & Ashland & 3 & WI \\
\hline 3065 & Hampshire & 27 & WV & 3109 & Barron & 5 & WI \\
\hline 3066 & Hancock & 29 & WV & 3110 & Bayfield & 7 & WI \\
\hline 3067 & Hardy & 31 & WV & 3111 & Brown & 9 & WI \\
\hline 3068 & Harrison & 33 & WV & 3112 & Buffalo & 11 & WI \\
\hline 3069 & Jackson & 35 & WV & 3113 & Burnett & 13 & WI \\
\hline 3070 & Jefferson & 37 & WV & 3114 & Calumet & 15 & WI \\
\hline 3071 & Kanawha & 39 & WV & 3115 & Chippewa & 17 & WI \\
\hline 3077 & Marshall & 51 & WV & 3121 & Door & 29 & WI \\
\hline 3078 & Mason & 53 & WV & 3122 & Douglas & 31 & WI \\
\hline 3079 & Mercer & 55 & WV & 3123 & Dunn & 33 & WI \\
\hline 3080 & Mineral & 57 & WV & 3124 & Eau Claire & 35 & WI \\
\hline 3081 & Mingo & 59 & WV & 3125 & Florence & 37 & WI \\
\hline 3082 & Monongalia & 61 & WV & 3126 & Fond du Lac & 39 & WI \\
\hline 3083 & Monroe & 63 & WV & 3127 & Forest & 41 & WI \\
\hline 3084 & Morgan & 65 & WV & 3128 & Grant & 43 & WI \\
\hline 3085 & Nicholas & 67 & WV & 3129 & Green & 45 & WI \\
\hline 3086 & Ohio & 69 & WV & 3130 & Green Lake & 47 & WI \\
\hline 3087 & Pendleton & 71 & WV & 3131 & Iowa & 49 & WI \\
\hline 3088 & Pleasants & 73 & WV & 3132 & Iron & 51 & WI \\
\hline 3089 & Pocahontas & 75 & WV & 3133 & Jackson & 53 & WI \\
\hline 3090 & Preston & 77 & WV & 3134 & Jefferson & 55 & WI \\
\hline 3091 & Putnam & 79 & WV & 3135 & Juneau & 57 & WI \\
\hline 3092 & Raleigh & 81 & WV & 3136 & Kenosha & 59 & WI \\
\hline 3093 & Randolph & 83 & WV & 3137 & Kewaunee & 61 & WI \\
\hline
\end{tabular}




\begin{tabular}{|c|c|c|c|c|c|c|c|}
\hline \multicolumn{4}{|c|}{ luCounty-Continued } & \multicolumn{4}{|c|}{ luCounty-Continued } \\
\hline $\begin{array}{l}\text { County } \\
\text { ID }\end{array}$ & County & $\begin{array}{l}\text { County } \\
\text { FIPSCode }\end{array}$ & $\begin{array}{l}\text { State } \\
\text { Code }\end{array}$ & $\begin{array}{l}\text { County } \\
\text { ID }\end{array}$ & County & $\begin{array}{l}\text { County } \\
\text { FIPSCode }\end{array}$ & $\begin{array}{l}\text { State } \\
\text { Code }\end{array}$ \\
\hline 3138 & La Crosse & 63 & WI & 3182 & Carbon & 7 & WY \\
\hline 3139 & Lafayette & 65 & WI & 3183 & Converse & 9 & WY \\
\hline 3140 & Langlade & 67 & WI & 3184 & Crook & 11 & WY \\
\hline 3141 & Lincoln & 69 & WI & 3185 & Fremont & 13 & WY \\
\hline 3142 & Manitowoc & 71 & WI & 3186 & Goshen & 15 & WY \\
\hline 3143 & Marathon & 73 & WI & 3187 & Hot Springs & 17 & WY \\
\hline 3144 & Marinette & 75 & WI & 3188 & Johnson & 19 & WY \\
\hline 3145 & Marquette & 77 & WI & 3189 & Laramie & 21 & WY \\
\hline 3146 & Menominee & 78 & WI & 3190 & Lincoln & 23 & WY \\
\hline 3147 & Milwaukee & 79 & WI & 3191 & Natrona & 25 & WY \\
\hline 3148 & Monroe & 81 & WI & 3192 & Niobrara & 27 & WY \\
\hline 3149 & Oconto & 83 & WI & 3193 & Park & 29 & WY \\
\hline 3150 & Oneida & 85 & WI & 3194 & Platte & 31 & WY \\
\hline 3151 & Outagamie & 87 & WI & 3195 & Sheridan & 33 & WY \\
\hline 3152 & Ozaukee & 89 & WI & 3196 & Sublette & 35 & WY \\
\hline 3153 & Pepin & 91 & WI & 3197 & Sweetwater & 37 & WY \\
\hline 3154 & Pierce & 93 & WI & 3198 & Teton & 39 & WY \\
\hline 3155 & Polk & 95 & WI & 3199 & Uinta & 41 & WY \\
\hline 3156 & Portage & 97 & WI & 3200 & Washakie & 43 & WY \\
\hline 3157 & Price & 99 & WI & 3201 & Weston & 45 & WY \\
\hline 3158 & Racine & 101 & WI & 3202 & Eastern District & 10 & AS \\
\hline 3159 & Richland & 103 & WI & 3203 & Manu'a District & 20 & AS \\
\hline 3160 & Rock & 105 & WI & 3204 & Rose Island & 30 & AS \\
\hline 3161 & Rusk & 107 & WI & 3205 & Swains Island & 40 & AS \\
\hline 3162 & St. Croix & 109 & WI & 3206 & Western District & 50 & AS \\
\hline 3163 & Sauk & 111 & WI & 3207 & Guam & 10 & $\mathrm{GU}$ \\
\hline 3164 & Sawyer & 113 & WI & 3208 & Northern Islands & 85 & MP \\
\hline 3165 & Shawano & 115 & WI & 3209 & Rota & 100 & MP \\
\hline 3166 & Sheboygan & 117 & WI & 3210 & Saipan & 110 & MP \\
\hline 3167 & Taylor & 119 & WI & 3211 & Tinian & 120 & MP \\
\hline 3168 & Trempealeau & 121 & WI & 3212 & Adjuntas & 1 & PR \\
\hline 3169 & Vernon & 123 & WI & 3213 & Aguada & 3 & PR \\
\hline 3170 & Vilas & 125 & WI & 3214 & Aguadilla & 5 & PR \\
\hline 3171 & Walworth & 127 & WI & 3215 & Aguas Buenas & 7 & PR \\
\hline 3172 & Washburn & 129 & WI & 3216 & Aibonito & 9 & PR \\
\hline 3173 & Washington & 131 & WI & 3217 & Anasco & 11 & PR \\
\hline 3174 & Waukesha & 133 & WI & 3218 & Arecibo & 13 & PR \\
\hline 3175 & Waupaca & 135 & WI & 3219 & Arroyo & 15 & PR \\
\hline 3176 & Waushara & 137 & WI & 3220 & Barceloneta & 17 & PR \\
\hline 3177 & Winnebago & 139 & WI & 3221 & Barranquitas & 19 & PR \\
\hline 3178 & Wood & 141 & WI & 3222 & Bayamon & 21 & PR \\
\hline 3179 & Albany & 1 & WY & 3223 & Cabo Rojo & 23 & PR \\
\hline 3180 & Big Horn & 3 & WY & 3224 & Caguas & 25 & PR \\
\hline 3181 & Campbell & 5 & WY & 3225 & Camuy & 27 & PR \\
\hline
\end{tabular}


IuCounty-Continued

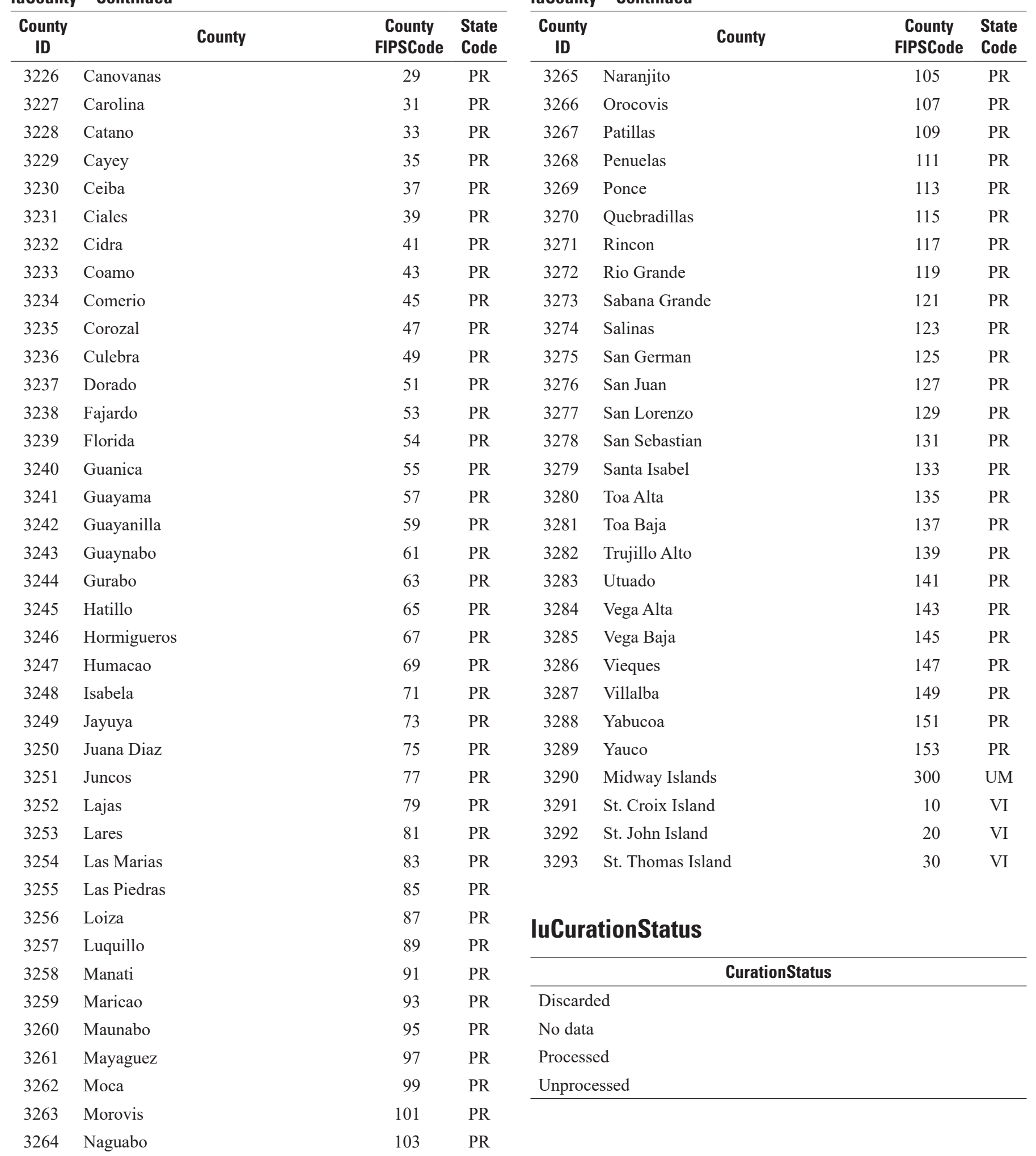




\section{luCWHRHabitat}

\begin{tabular}{|c|c|c|c|}
\hline $\begin{array}{l}\text { CWHRHabitat } \\
\text { Code }\end{array}$ & CWHRHabitat & $\begin{array}{c}\text { CWHRHabitat } \\
\text { GroupID }\end{array}$ & CWHRHab Desc \\
\hline ADS & Alpine Dwarf-Shrub & 9 & \\
\hline AGS & Annual Grass & 14 & \\
\hline $\mathrm{ASC}$ & Alkali Desert Scrub & 13 & \\
\hline ASP & Aspen & 3 & \\
\hline BAR & Barren & 22 & \\
\hline BBR & Bitterbrush & 10 & \\
\hline BOP & Blue Oak-Foothill Pine & 7 & \\
\hline BOW & Blue Oak Woodland & 7 & \\
\hline COW & Coastal Oak Woodland & 7 & \\
\hline $\mathrm{CPC}$ & Closed-Cone Pine-Cypress & 6 & \\
\hline CRC & Chamise-Redshank Chaparral & 11 & \\
\hline $\mathrm{CSC}$ & Coastal Scrub & 12 & \\
\hline DFR & Douglas Fir & 1 & \\
\hline DGR & Dryland Grain Crops & 20 & \\
\hline DOR & Deciduous Orchard & 21 & \\
\hline DRI & Desert Riparian & 8 & \\
\hline DSC & Desert Scrub & 13 & \\
\hline DSS & Desert Succulent Shrub & 13 & \\
\hline DSW & Desert Wash & 13 & \\
\hline EOR & Evergreen Orchard & 21 & \\
\hline EPN & Eastside Pine & 2 & \\
\hline EST & Estuarine & 18 & \\
\hline EUC & Eucalyptus & 21 & \\
\hline FEW & Fresh Emergent Wetland & 15 & \\
\hline IGR & Irrigated Grain Crops & 20 & \\
\hline IRF & Irrigated Row And Field Crops & 20 & \\
\hline IRH & Irrigated Hayfield & 20 & \\
\hline JPN & Jeffrey Pine & 2 & \\
\hline JST & Joshua Tree & 5 & \\
\hline JUN & Juniper & 2 & \\
\hline $\mathrm{KMC}$ & Klamath Mixed Conifer & 1 & \\
\hline LAC & Lacustrine & 17 & \\
\hline LPN & Lodgepole Pine & 3 & \\
\hline LSG & Low Sage & 10 & \\
\hline MAR & Marine & 19 & \\
\hline $\mathrm{MCH}$ & Mixed Chaparral & 11 & \\
\hline $\mathrm{MCP}$ & Montane Chaparral & 11 & \\
\hline MHC & Montane Hardwood-Conifer & 4 & \\
\hline MHW & Montane Hardwood & 4 & \\
\hline MRI & Montane Riparian & 8 & \\
\hline PAS & Pasture & 14 & \\
\hline PGS & Perennial Grass & 14 & \\
\hline PJN & Pinyon-Juniper & 5 & \\
\hline POS & Palm Oasis & 8 & \\
\hline
\end{tabular}




\begin{tabular}{clc} 
luCWHRHabitat-Continued & \multicolumn{1}{c}{ CWHRHabitat } \\
$\begin{array}{c}\text { CWHRHabitat } \\
\text { Code }\end{array}$ & \multicolumn{1}{c}{$\begin{array}{c}\text { CWHRHabitat } \\
\text { GrouplD }\end{array}$} & CWHRHab Desc \\
\hline PPN & Ponderosa Pine & 4 \\
RDW & Redwood & 1 \\
RFR & Red Fir & 3 \\
RIC & Rice & 20 \\
RIV & Riverine & 16 \\
SCN & Subalpine Conifer & 3 \\
SEW & Saline Emergent Wetland & 15 \\
SGB & Sagebrush & 10 \\
SMC & Sierran Mixed Conifer & 4 \\
URB & Urban & 22 \\
VIN & Vineyard & 21 \\
VOW & Valley Oak Woodland & 7 \\
VRI & Valley Foothill Riparian & 8 \\
WFR & White Fir & 4 \\
WTM & Wet Meadow & 15 \\
\hline
\end{tabular}

\section{IuDensityUnit}

\section{DensityUnit}

Acres

Hectares

Square feet

Square meters

\section{IuDetectionMethod}

\begin{tabular}{ccl}
\hline DetectionMethodID & DetectionMethodCode & \\
\hline 1 & H C & Heard calling \\
2 & VIS & Visual \\
3 & HND & Handled \\
4 & H S & Heard singing \\
5 & WHT & Whitting \\
6 & TRK & Track \\
7 & SCT & Scat \\
8 & HAR & Hair \\
9 & SIN & Miscellaneous sign \\
10 & CAP & Captured \\
11 & UNK & Unknown \\
12 & DOG & Scent detected by dog \\
13 & SAT & Satellite/Telemetry \\
14 & VSA & Visual, aerial \\
15 & VSL & Visual, land \\
16 & RTS & Radio transmitter signal \\
17 & H O & Heard, other \\
18 & HAV & Heard and visually confirmed \\
\hline & &
\end{tabular}




\section{luDirection}

\begin{tabular}{cll}
\hline DirectionCode & & Direction \\
\hline E & East \\
N & North \\
NA & Not applicable \\
NE & Northeast \\
NW & Northwest \\
S & South \\
SE & Southeast \\
SW & Southwest \\
U & Unknown \\
W & West
\end{tabular}

\section{luDisposition}

\begin{tabular}{ll}
\hline \multicolumn{1}{c}{ Disposition } \\
\hline Alive \\
Dead \\
Moribund
\end{tabular}

\section{luDistanceToType}

\begin{tabular}{l}
\hline \multicolumn{1}{c}{ Type } \\
\hline Geological feature \\
Other taxon observation \\
This observation from observer \\
Water
\end{tabular}

\section{luEstMethod}

\begin{tabular}{ll}
\hline & EstMethod \\
\hline Estimated & \\
Measured \\
Previously known
\end{tabular}

\section{luExtentInundation}

\begin{tabular}{cl}
\hline $\begin{array}{c}\text { ExtentInundation } \\
\text { ID }\end{array}$ & \multicolumn{1}{c}{ ExtentInundation } \\
\hline 0 & 0 percent dry \\
1 & $1 \sim 5$ percent inundated \\
2 & $6 \sim 25$ percent inundated \\
3 & $26 \sim 50$ percent inundated \\
4 & $51 \sim 75$ percent inundated \\
5 & $76 \sim 100$ percent inundated \\
\hline
\end{tabular}

\section{luFacilityType}

\begin{tabular}{cll}
\hline FacilityTypeID & FacilityType & Description \\
\hline 1 & Office building & \\
2 & Restroom \\
3 & Parking lot \\
4 & Open space \\
99 & Other \\
\hline
\end{tabular}

luFate

\begin{tabular}{ll}
\hline & Fate \\
\hline Collected & \\
Escaped & \\
Released & \\
\hline
\end{tabular}

\section{luFeature Type}

\begin{tabular}{ll}
\hline & FeatureType \\
\hline Basking & \\
Breeding & \\
General suitability & \\
Nesting & \\
\hline
\end{tabular}

\section{luFocalSpeciesPurpose}

\section{FocalSpeciesPurpose}

Active management - control/remove

Active management - enhancement

Surveys - abundance

Surveys - inventory

Surveys - occupancy

\section{luFreqofTravel}

\begin{tabular}{ll}
\hline \multicolumn{1}{c}{ FrequencyTraveled } \\
\hline Closed \\
Daily \\
Monthly \\
Other \\
Unknown \\
Weekly \\
Yearly
\end{tabular}




\section{luFundingAction}

\begin{tabular}{lcc}
\hline FundingActionID $\quad$ FundingAction & Description \\
\hline No values in look up table as of January 2021. & \\
\hline
\end{tabular}

\section{luFundingType}

\begin{tabular}{cc}
\hline FundingTypeID & FundingType \\
\hline 1 & Transnet EMP Land Management Grant \\
\hline
\end{tabular}

\section{luGender}

\begin{tabular}{cll}
\hline GenderID & \multicolumn{1}{c}{ Gender } & \multicolumn{1}{c}{ Description } \\
\hline 1 & Male & \\
2 & Female & \\
3 & Hermaphrodite & \\
4 & Both & Male(s) and female(s) included \\
98 & Not applicable & \\
99 & Unknown & \\
\hline
\end{tabular}

\section{luGroundCover}

\begin{tabular}{clc}
\hline GroundCoverID & \multicolumn{1}{c}{ GroundCover } & GroundCoverCode \\
\hline 1 & None/Bare & $\mathrm{N}$ \\
3 & Duff & $\mathrm{D}$ \\
4 & Small slash & $\mathrm{SS}$ \\
5 & Large slash & LS \\
6 & Medium log & LM \\
7 & Large log & LL \\
8 & Annual grass & Ang \\
9 & Perennial grass & Png \\
10 & Forb & F \\
11 & Pine straw & PS \\
12 & Broad-leaved & BL \\
13 & Woody/Bark & W \\
14 & Shrubs and trees & ST \\
\hline
\end{tabular}

\section{luHabitatFeatureType}

\begin{tabular}{|c|c|c|}
\hline $\begin{array}{l}\text { HabitatFeature } \\
\text { TypelD }\end{array}$ & HabitatFeature Type & Description \\
\hline 1 & Tunnel & Man-made shaft or tunnel below ground. \\
\hline 3 & Burrow & Subterranean tunnel dug, or significantly enhanced, by an animal. \\
\hline 4 & Rocks & An area dominated by rocks. \\
\hline 7 & Mineral lick & A mineral-rich area used by animals for meeting dietary needs. \\
\hline 8 & Duff layer & $\begin{array}{l}\text { Layer of decomposing organic material below the surface of the ground but above the } \\
\text { mineral soil. }\end{array}$ \\
\hline 9 & Dead/Down wood & An area characterized by large amounts of dead and down woody debris. \\
\hline 13 & Wetland/Vernal pool & \\
\hline 14 & Spring/Seep & \\
\hline 15 & Fen & \\
\hline 16 & Wet meadow & \\
\hline 17 & Swamp & \\
\hline 18 & Creek/Brook/Stream/River & \\
\hline 19 & Oxbow/Backwaters/ Floodplain & \\
\hline 20 & Beaver pond & \\
\hline 21 & $\log$ & $\begin{array}{l}\text { Associated with a dead part of the main stem of a tree which is lying on the ground } \\
(>8 \text { inches diameter and }>10 \text { feet in length). }\end{array}$ \\
\hline
\end{tabular}




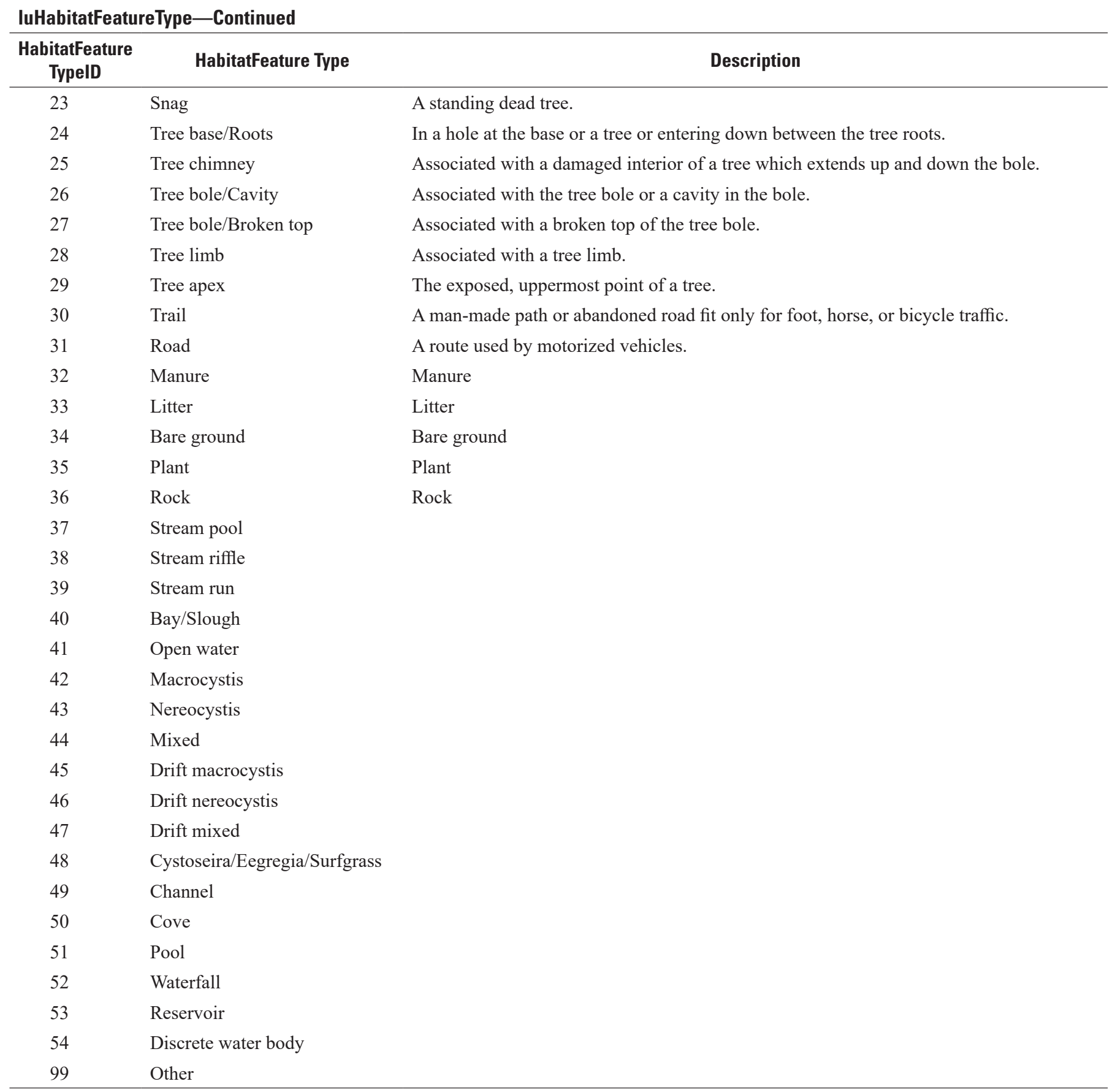

\section{luHabitatObsVegContext}

\begin{tabular}{ll}
\hline \multicolumn{1}{c}{ Context } \\
\hline Adjacent \\
Aquatic \\
Riparian \\
Upland \\
\hline
\end{tabular}




\section{luHabitatVegetationType}

\begin{tabular}{clc}
\hline $\begin{array}{c}\text { HabitatVegetation } \\
\text { TypelD }\end{array}$ & HabitatVegetation Type & $\begin{array}{c}\text { HabitatVegetation } \\
\text { TypeCode }\end{array}$ \\
\hline 1 & $\begin{array}{c}\text { Submerged aquatic } \\
\text { vegetation }\end{array}$ & SAV \\
2 & Emergents & $\mathrm{E}$ \\
3 & Other herbaceous & $\mathrm{O}$ \\
4 & Shrub/Sapling & $\mathrm{Shr}$ \\
5 & Trees & $\mathrm{T}$ \\
6 & Open ground & $\mathrm{GND}$ \\
7 & Woody debris/Leaf litter & $\mathrm{WL}$ \\
8 & Herbs-Forbs & $\mathrm{HF}$ \\
9 & Non-native grass & $\mathrm{NNG}$ \\
10 & Native bunch grass & $\mathrm{NBG}$ \\
11 & Floating algal mat & FAM \\
12 & Inhospitable & $\mathrm{INH}$
\end{tabular}

\section{luHabSuitability}

\begin{tabular}{cll}
\hline HSID & & Suitability \\
\hline 1 & High quality & \\
2 & Suitable & \\
3 & Marginal & \\
4 & Not suitable & \\
\hline
\end{tabular}

\section{luHeightClass}

\begin{tabular}{ll}
\hline \multicolumn{1}{c}{ HeightClass } \\
\hline Herb \\
Over $75 \mathrm{~cm}$ \\
Shrub \\
Tree \\
Under $75 \mathrm{~cm}$ \\
\hline
\end{tabular}

\section{luHistoricaIPresence}

\begin{tabular}{ll}
\hline & HistoricalPresence \\
\hline Exotic & \\
Invasive & \\
Native & \\
\hline
\end{tabular}

\section{IulrrigationMethods}

\begin{tabular}{ccc}
\hline IrrigationMethodID $\quad$ IrrigationMethod & Description \\
\hline No values in look up table as of January 2021. & \\
\hline
\end{tabular}

\section{luKeywordType}

\begin{tabular}{ll}
\hline & Type \\
\hline ARMI keyword & \\
ARMI place name & \\
ARMI topic & \\
ARMI website & \\
\hline
\end{tabular}

\section{luLandUse}

\begin{tabular}{|c|c|c|}
\hline LandUseID & LandUse & LandUseCode \\
\hline 1 & Agriculture & A \\
\hline 2 & Industrial & I \\
\hline 3 & Woodland/Forest & $\mathrm{W}$ \\
\hline 4 & Meadow/Marsh & M \\
\hline 5 & Residential & $\mathrm{R}$ \\
\hline 98 & Not applicable & NA \\
\hline 99 & Unknown & $\mathrm{U}$ \\
\hline
\end{tabular}

\section{luLifeStage}

\begin{tabular}{cll}
\hline LifeStagelD & \multicolumn{1}{c}{ LifeStage } & Description \\
\hline 1 & Adult & Fully developed and sexually mature \\
4 & Juvenile/Young & $\begin{array}{l}\text { Not yet fully developed and sexually mature } \\
\text { Age could not be determined }\end{array}$ \\
6 & Age Unknown & $\begin{array}{l}\text { A reproductive body consisting of an ovum together with its nutritive and protective envelopes and } \\
\text { having the capacity to develop into a new individual capable of independent existence }\end{array}$ \\
7 & Egg/Embryo & The newly hatched young of any bird \\
9 & Chick & A young bird that has not abandoned the nest \\
10 & Fledgling & A bird that is ready for flight or independent activity \\
12 & Hatchling & A recently hatched animal \\
13 & Larva & The early form of an animal that at birth or hatching is fundamentally unlike its parent and must \\
& & metamorphose before assuming the adult characteristics
\end{tabular}


IuLifeStage-Continued

\begin{tabular}{|c|c|c|}
\hline LifeStagelD & LifeStage & Description \\
\hline 15 & Fry & A larval fish that has just absorbed its yolk sac and emerged from the gravel \\
\hline 16 & Smolt & An anadromous fish that becomes silvery and migrates to the sea \\
\hline 18 & Pupa & The inactive stage in the metamorphosis of many insects \\
\hline 19 & Metamorph & Metamorphosis is under way; Gosner $42-46$, forelimbs emerge to complete metamorphosis \\
\hline 22 & After hatching year & \\
\hline 23 & Hatching year & \\
\hline 24 & Second year & \\
\hline 25 & Independent & Adult and subadult sea otter \\
\hline 30 & Gosner 1 & Fertilization - embryo \\
\hline 31 & Gosner 2 & Gray crescent - embryo \\
\hline 32 & Gosner 3 & 2-cell - embryo \\
\hline 33 & Gosner 4 & 4-cell - embryo \\
\hline 34 & Gosner 5 & 8-cell - embryo \\
\hline 35 & Gosner 6 & 16-cell - embryo \\
\hline 36 & Gosner 7 & 32-cell - embryo \\
\hline 37 & Gosner 8 & Midcleavage - embryo \\
\hline 38 & Gosner 9 & Late cleavage - embryo \\
\hline 44 & Gosner 15 & Elongation, rotation - embryo \\
\hline 45 & Gosner 16 & Neural tube, gill plates - embryo \\
\hline 46 & Gosner 17 & Tail bud, adhesive gland - embryo \\
\hline 47 & Gosner 18 & Muscular response, olfactory pits - embryo \\
\hline 48 & Gosner 19 & Heartbeat, gill buds - embryo \\
\hline 49 & Gosner 20 & Gill circulation, tail elongation - hatchling \\
\hline 50 & Gosner 21 & Cornea transplant, mouth opens - hatchling \\
\hline 51 & Gosner 22 & Tail fins transparent, fin circulation - hatchling \\
\hline 52 & Gosner 23 & Labia and teeth differentiate, operculum covers gill bases - Hatchling \\
\hline 53 & Gosner 24 & External gills atrophy, operculum closes on right - hatchlings \\
\hline 54 & Gosner 25 & Mouthparts obvious, spiracle forms on left - hatchling \\
\hline 55 & Gosner 26 & $\mathrm{~L}<.5 \mathrm{D}-$ larvae \\
\hline 56 & Gosner 27 & $\mathrm{~L}>=.5 \mathrm{D}-$ larvae \\
\hline 57 & Gosner 28 & $\mathrm{~L}>=\mathrm{D}-$ larvae \\
\hline 58 & Gosner 29 & $\mathrm{~L}>=1.5 \mathrm{D}-$ larvae \\
\hline 59 & Gosner 30 & $\mathrm{~L}=2 \mathrm{D}-$ larvae \\
\hline
\end{tabular}




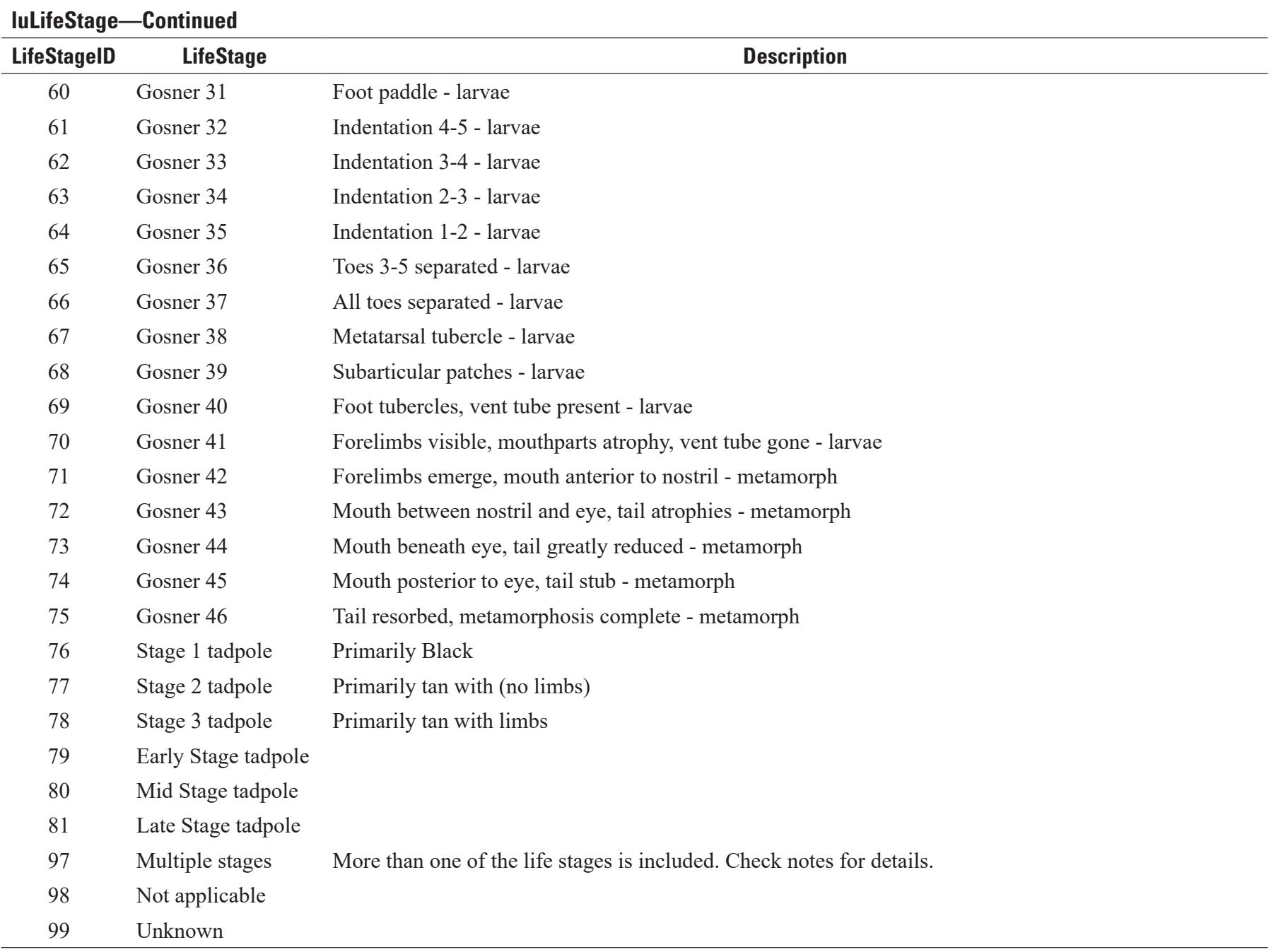

\section{IuLocationClass}

\begin{tabular}{llll}
\hline Class & Type & EstError & MsgsPerSatellitePass \\
\hline $\mathrm{G}$ & GPS & $<100 \mathrm{~m}$ & 1 message or more \\
L3 & Argos & $<250 \mathrm{~m}$ & 4 messages or more \\
L2 & Argos & $250 \mathrm{~m}<<500 \mathrm{~m}$ & 4 messages or more \\
L1 & Argos & $500 \mathrm{~m}<<1500 \mathrm{~m}$ & 4 messages or more \\
L0 & Argos & $>1500 \mathrm{~m}$ & 4 messages or more \\
LA & Argos & No accuracy estimation & 3 messages \\
LB & Argos & No accuracy estimation & 2 messages \\
LZ & Argos & Invalid location (for Service Plus/Auxiliary loc processing) \\
DP & & deployment &
\end{tabular}

\section{luMaintPointType}

\begin{tabular}{cc}
\hline MaintPointType & Decription \\
\hline No values in look up table as of January 2021. & \\
\hline
\end{tabular}

\section{luMaintType}

\begin{tabular}{clc}
\hline MaintTypeID & MaintType & \multicolumn{1}{c}{ Description } \\
\hline 1 & Fence & Vehicle barriers \\
\hline
\end{tabular}


luMaterialType

\begin{tabular}{ccc}
$\begin{array}{c}\text { MaterialType } \\
\text { ID }\end{array}$ & $\begin{array}{c}\text { Material } \\
\text { Type }\end{array}$ & Description \\
\hline 1 & Steel & Rigid steel vehicle barriers \\
\hline & & \\
IuMgtProjectStatus & \\
\hline \multicolumn{2}{c}{ Status } & Description \\
\hline Completed & \\
Started & \\
Started & & \\
\hline
\end{tabular}

\section{luMgtType}

\begin{tabular}{cll}
\hline MgtTypeID & \multicolumn{1}{c}{ MgtType } & Description \\
\hline 2 & Access control & \\
3 & Habitat restoration & \\
\hline
\end{tabular}

\section{luMilestoneType}

\section{MilestoneTypeID}

No values in look up table as of January 2021

\section{luMonitoringType}

\begin{tabular}{ll} 
MonitoringTypeID $\quad$ MonitoringType & Description \\
\hline No values in look up table as of January 2021. & \\
\hline
\end{tabular}

\section{luMoonPhase}

\begin{tabular}{cl}
\hline MoonPhaseCode & MoonPhase \\
\hline 3Q & Three quarter \\
F & Full \\
H & Half \\
N & New \\
NA & Not applicable \\
NV & Not visible \\
Q & Quarter \\
U & Unknown \\
V & Visible \\
\hline
\end{tabular}

\section{luMSCPLandDesignation}

MSCPLandDesignation

\section{Core}

Corridor

\begin{tabular}{ll}
\hline \multicolumn{1}{c}{ MSCPLandDesignation } \\
\hline Core \\
Corridor
\end{tabular}

\section{luObGrowthForm}

$\begin{array}{ll}\text { Supplier } & \text { ObGrowthForm } \\ \text { Annual grass } & \\ \text { Forb } & \\ \text { Forb/Herb } \\ \text { Grass } \\ \text { Herb } \\ \text { Perennial Grass } \\ \text { Shrub } \\ \text { Shrub/Tree } \\ \text { Tree }\end{array}$

\section{luObsTiming}

\begin{tabular}{ll}
\hline \multicolumn{1}{c}{ ObsTiming } \\
\hline End \\
Max \\
Mid \\
Min \\
NA \\
Start
\end{tabular}

\section{luParameterType}

\begin{tabular}{ll}
\hline \multicolumn{1}{c}{ Parameter } \\
\hline Abundance \\
Eggmass count \\
Occupancy \\
\hline \\
luPhenology \\
\hline \\
\hline Early \\
Late \\
Peak
\end{tabular}

\section{luPhotoQuality}

\begin{tabular}{cl}
\hline PhotoQualitylD & \multicolumn{1}{c}{ PhotoQuality } \\
\hline 1 & High/Media \\
2 & Medium/Presentation acceptable \\
3 & Low/Documentation only \\
\hline
\end{tabular}




\section{luPhotoType}

\begin{tabular}{cl}
\hline PhotoTypeID & \multicolumn{1}{c}{ PhotoType } \\
\hline 1 & Original digital file \\
2 & Duplicate digital file \\
3 & Original color transparency \\
4 & Duplicate color transparency \\
5 & Original negative \\
6 & Duplicate negative \\
7 & Print \\
8 & Xerox type copy or fax \\
9 & Digital from film \\
\hline
\end{tabular}

\section{luPlantingForm}

\begin{tabular}{lcc}
\hline PlantingFormID & PlantingForm & Description \\
\hline No values in look up table as of January 2021. & \\
\hline
\end{tabular}

\section{IuPlantObDetailType}

\begin{tabular}{|c|c|}
\hline DetailType & Description \\
\hline Individual & Single plant \\
\hline Patch/Stand & $\begin{array}{l}\text { Cohesive grouping of trees (stand) or a grouping of } \\
\text { herbs, shrubs, succulents (patch) }\end{array}$ \\
\hline
\end{tabular}

\section{luPlantSizeClass}

\begin{tabular}{c}
\hline PlantSizeClass \\
\hline 25 \\
50 \\
75 \\
100 \\
300 \\
1000 \\
1500 \\
\hline
\end{tabular}

\section{IuPopulationDesignation}

\begin{tabular}{ll}
\hline & PopulationDesignation \\
\hline Major & \\
Minor & \\
\hline
\end{tabular}

\section{IuPreservationMethod}

\begin{tabular}{cl}
\hline PresMethodID & \multicolumn{1}{c}{ Description } \\
\hline 1 & 95 percent EtOH \\
2 & 70 percent EtOH \\
3 & Formalin
\end{tabular}

\begin{tabular}{|c|c|}
\hline PresMethodID & Description \\
\hline 4 & Frozen \\
\hline 5 & Desiccated \\
\hline 6 & Pinned \\
\hline
\end{tabular}

\section{luProjectCategory}

\begin{tabular}{lc}
\hline ProjectCategory & \multicolumn{1}{c}{ Description } \\
\hline Data migration & $\begin{array}{c}\text { These studies are related by inclusion within the } \\
\text { same data migration effort. }\end{array}$ \\
$\begin{array}{l}\text { Related by } \\
\text { theme }\end{array}$ & $\begin{array}{c}\text { These studies are related by a common scientific } \\
\text { theme; such as by a common program or } \\
\text { funding source. }\end{array}$ \\
\hline
\end{tabular}

\section{luPubicSymphysis}

\begin{tabular}{lll}
\hline PubSymID & & Description \\
\hline OPN & Open & \\
SOP & Slightly open & \\
CLS & Closed & \\
UNK & Unknown & \\
\hline
\end{tabular}

\section{IuRefugiaContext}

\begin{tabular}{l}
\hline \multicolumn{1}{c}{ Context } \\
\hline Aquatic \\
Bank \\
\hline \\
luRefugiaType \\
\hline \\
\hline Emergent veg \\
Floating material \\
Rock crevasses \\
Submergent veg \\
Tree roots \\
Undercuts \\
Woody debris \\
\hline
\end{tabular}

\section{luRelativeAbundance}

\begin{tabular}{ll}
\hline & RelativeAbundance \\
\hline Few & \\
Many & \\
None & \\
\hline
\end{tabular}




\section{luRemovallntroductionValues}

\begin{tabular}{ccc}
\hline $\begin{array}{c}\text { Removallntro } \\
\text { ValuelD }\end{array}$ & $\begin{array}{c}\text { Removallntroduction } \\
\text { Value }\end{array}$ & Description \\
\hline No values in look up table as of January 2021. & \\
\hline
\end{tabular}

\section{luReturnExpected}

\begin{tabular}{ll}
\hline & ReturnExpected \\
\hline No & \\
Returned & \\
Yes & \\
\hline
\end{tabular}

\section{luRoadSurface}

\begin{tabular}{cll}
\hline RoadSurfaceCode & RoadSurface & \multicolumn{1}{c}{ Description } \\
\hline 1 & Dry & $\begin{array}{c}\text { Dry with only few moist } \\
\text { patches } \\
\text { Damp with no draining or } \\
\text { standing water } \\
3\end{array}$ \\
& Wamp & $\begin{array}{l}\text { Wet with draining or standing } \\
\text { water }\end{array}$ \\
4 & Sheeting & $\begin{array}{c}\text { Sheeting with water flowing off } \\
\text { the surface }\end{array}$ \\
\hline
\end{tabular}

\section{luRoadsTrailsAccess}

\begin{tabular}{ll}
\hline & Access \\
\hline Other & \\
Private & \\
Public & \\
Utility & \\
\hline
\end{tabular}

\section{luRoadsTrailsEdge}

\begin{tabular}{ll}
\hline & Edge \\
\hline Curb & \\
Fence & \\
K-rail & \\
None & \\
Other & \\
\hline
\end{tabular}

\section{luRoadsTrailsType}

\begin{tabular}{ll}
\hline & Type \\
\hline Dirt road & \\
Firebreak & \\
Gravel road & \\
Paved road & \\
Trail & \\
\hline
\end{tabular}

\section{luSedimentationChemical}

Sed

\section{Sedimentation ChemicallD}

Sedimentation Chemical

Description

1

Aluminum to nearest 0.005 percent

NA

Antimony to nearest $0.10 \mu \mathrm{g} / \mathrm{g}$

NA

Arsenic to nearest $0.1 \mu \mathrm{g} / \mathrm{g}$

NA

Barium to nearest $1.0 \mu \mathrm{g} / \mathrm{g}$

NA

Beryllium to nearest $0.10 \mu \mathrm{g} / \mathrm{g}$

NA

Bismuth to nearest $1.0 \mu \mathrm{g} / \mathrm{g}$

NA

Cadmium to nearest $0.10 \mu \mathrm{g} / \mathrm{g}$

NA

Calcium to nearest 0.005 percent

NA

Cerium to nearest $1.0 \mu \mathrm{g} / \mathrm{g}$

NA

Chromium to nearest $1.0 \mu \mathrm{g} / \mathrm{g}$

NA

Cobalt to nearest $1.0 \mu \mathrm{g} / \mathrm{g}$

NA

Copper to nearest $1.0 \mu \mathrm{g} / \mathrm{g}$

NA

Europium to nearest $1.0 \mu \mathrm{g} / \mathrm{g}$

NA

Gallium to nearest $1.0 \mu \mathrm{g} / \mathrm{g}$

NA

Gold to nearest $1 \mu \mathrm{g} / \mathrm{g}$

NA

Holmium to nearest $1 \mu \mathrm{g} / \mathrm{g}$

NA

Inorganic carbon to nearest 0.01 percent

Iron to nearest 0.005 percent

NA

Lanthanum to nearest $1.0 \mu \mathrm{g} / \mathrm{g}$

NA

Lead to nearest $1.0 \mu \mathrm{g} / \mathrm{g}$

NA

Lithium to nearest $1.0 \mu \mathrm{g} / \mathrm{g}$

NA

Magnesium to nearest 0.005 percent

NA

Manganese to nearest $4.0 \mu \mathrm{g} / \mathrm{g}$

NA

Mercury to nearest $0.020 \mu \mathrm{g} / \mathrm{g}$

NA

Methyl mercury to nearest $0.020 \mu \mathrm{g} / \mathrm{g}$

NA

Molybdenum to nearest $0.50 \mu \mathrm{g} / \mathrm{g}$

NA

Neodymium to nearest $1.0 \mu \mathrm{g} / \mathrm{g}$

NA

Nickel to nearest $2.0 \mu \mathrm{g} / \mathrm{g}$

NA

Niobium to nearest $4.0 \mu \mathrm{g} / \mathrm{g}$

NA

Organic carbon to nearest 0.01 percent

NA

Phosphorus to nearest 0.005 percent

NA

Potassium to nearest 0.005 percent

NA

Scandium to nearest $2.0 \mu \mathrm{g} / \mathrm{g}$

NA

Selenium to nearest $0.1 \mu \mathrm{g} / \mathrm{g}$

NA

Silver to nearest $0.10 \mu \mathrm{g} / \mathrm{g}$

NA

Sodium to nearest 0.005 percent

NA

Strontium to nearest $2.0 \mu \mathrm{g} / \mathrm{g}$

NA

Sulfur to nearest 0.05 percent

NA

Tantalum $1.0 \mu \mathrm{g} / \mathrm{g}$

NA

Thallium $1.0 \mu \mathrm{g} / \mathrm{g}$

NA

Thorium $1.0 \mu \mathrm{g} / \mathrm{g}$

NA

Tin $1.0 \mu \mathrm{g} / \mathrm{g}$ 
IuSedimentationChemical-Continued

\begin{tabular}{clc}
\hline $\begin{array}{c}\text { Sedimentation } \\
\text { ChemicallD }\end{array}$ & \multicolumn{1}{c}{ Sedimentation Chemical } & Description \\
\hline 43 & Titanium 0.005 percent & NA \\
44 & Total carbon 0.01 percent & NA \\
45 & Uranium $0.1 \mu \mathrm{g} / \mathrm{g}$ & $\mathrm{NA}$ \\
46 & Vanadium $2.0 \mu \mathrm{g} / \mathrm{g}$ & $\mathrm{NA}$ \\
47 & Ytterbium $1.0 \mu \mathrm{g} / \mathrm{g}$ & $\mathrm{NA}$ \\
48 & Yttrium $1.0 \mu \mathrm{g} / \mathrm{g}$ & $\mathrm{NA}$ \\
49 & Zinc $2.0 \mu \mathrm{g} / \mathrm{g}$ & $\mathrm{NA}$ \\
\hline
\end{tabular}

\section{luSESFRelationship}

\begin{tabular}{ll}
\hline \multicolumn{1}{c}{ Relationship } \\
\hline Actual end \\
Actual start \\
Other \\
Special
\end{tabular}

\section{luSFCLDBMethod}

Method

No values in look up table as of January 2021.

\section{luShwear}

\begin{tabular}{|c|c|}
\hline Shwear & Description \\
\hline 1 & $<60 \mathrm{~mm}$ MCL; 95 percent of each nonmarginal scute composed of areola. Granulations on areola distinct. \\
\hline 2 & Total amount of areola worn smooth is less than or equivalent to 1.5 areolae. \\
\hline 4 & $\begin{array}{l}\text { Chipping/Flaking of growth rings limited and primarily on inner rings next to areolae. Growth rings distinct, in good condition. } \\
<50 \text { percent of a line across the width/length of scutes overlies smooth worn areas (abbreviated). }\end{array}$ \\
\hline 6 & $\begin{array}{l}\text { See class } 5 \text { description except depressions present on eight or fewer scutes, primarily on the second and third costals and second } \\
\text { and third vertebrals; generally patchy in distribution. }\end{array}$ \\
\hline 7 & $\begin{array}{l}\text { See class } 5 \text { description except depressions present on nine or more scutes and generally much more uniform, often involving all } \\
\text { but the edges of entire scutes. }\end{array}$ \\
\hline
\end{tabular}

\section{IuSiteQuality}

\begin{tabular}{cll}
\hline SiteQualitylD & SiteQuality & SiteQualityDescription \\
\hline 1 & Excellent & \\
2 & Good & \\
3 & Fair & \\
4 & Poor & \\
5 & High & \\
6 & Marginal & \\
\hline
\end{tabular}

\section{IuSiteRelationshipType}

\begin{tabular}{ll}
\hline & RelationshipType \\
\hline Downstream limit & \\
Eastern-most &
\end{tabular}

\begin{tabular}{l}
\hline IuSiteRelationshipType-Continued \\
\hline RelationshipType \\
\hline NE-most \\
Northern-most \\
NW-most \\
SE-most \\
Southern-most \\
SW-most \\
Upstream limit \\
Western-most
\end{tabular}

\section{luSiteThreatType}

\begin{tabular}{lll} 
SiteThreatTypeID & SiteThreatType & Description \\
\hline No values in look up table as of January 2021. & \\
\hline
\end{tabular}




\section{luSkyObservation}

\begin{tabular}{cll}
\hline Sky0bservationID & \multicolumn{1}{c}{ Sky0bservation } & \multicolumn{1}{c}{ Description } \\
\hline 1 & Clear or few clouds & Appoximately 0-10 percent cloudy \\
2 & Partly cloudy or variable & Appoximately 11-80 percent cloudy \\
3 & Cloudy or overcast & Appoximately $>80$ percent cloudy \\
4 & Fog & NA \\
5 & Mist or drizzle & NA \\
6 & Showers or light rain & NA \\
7 & Heavy rain & NA \\
8 & Sleet or hail & NA \\
9 & Snow & NA \\
98 & Not applicable & NA \\
99 & Unknown & NA \\
\hline
\end{tabular}

\section{luSoilMethod}

\begin{tabular}{ll}
\hline & Method \\
\hline Measured \\
\hline
\end{tabular}

\section{IuSoilTextureClass}

\begin{tabular}{l}
\hline TextureClass \\
\hline Clay \\
Clay loam \\
Loam \\
Loamy sand \\
Sand \\
Sandy clay \\
Sandy clay loam \\
Sandy loam \\
Silt \\
Silty clay \\
Silty clay loam \\
Silty loam
\end{tabular}

\section{luSoilUnit}

\begin{tabular}{l} 
Unit \\
\hline Grains \\
Grams \\
Milliliters \\
Percent mass \\
Percent volume
\end{tabular}

\section{luSourceType}

\begin{tabular}{cl}
\hline SourceTypeID & \multicolumn{1}{c}{ Description } \\
\hline 1 & Directly from survey; MTX is native DBS \\
2 & Migrated from other raw-date database/source \\
3 & Derived from report or other summarization \\
\hline
\end{tabular}

\section{luSpeciesDetected}

\begin{tabular}{cll} 
SDCode & Description \\
0 & Not detected & \\
1 & Detected \\
2 & Not surveyed for & \\
\hline
\end{tabular}

\section{luState}

\begin{tabular}{cl}
\hline StateCode & \multicolumn{1}{c}{ State } \\
\hline AK & Alaska \\
AL & Alabama \\
AR & Arkansas \\
AS & American Samoa \\
AZ & Arizona \\
CA & California \\
CO & Colorado \\
CT & Connecticut \\
DC & Washington, DC \\
DE & Delaware \\
FL & Florida \\
GA & Georgia \\
GU & Guam \\
HI & Hawaii \\
IA & Iowa
\end{tabular}




\begin{tabular}{|c|c|}
\hline StateCode & State \\
\hline ID & Idaho \\
\hline IL & Illinois \\
\hline IN & Indiana \\
\hline KS & Kansas \\
\hline KY & Kentucky \\
\hline LA & Louisiana \\
\hline MA & Massachusetts \\
\hline MD & Maryland \\
\hline ME & Maine \\
\hline $\mathrm{MH}$ & Marshall Islands \\
\hline MI & Michigan \\
\hline $\mathrm{MN}$ & Minnesota \\
\hline MO & Missouri \\
\hline MP & Northern Mariana Islands \\
\hline MS & Mississippi \\
\hline MT & Montana \\
\hline $\mathrm{NC}$ & North Carolina \\
\hline ND & North Dakota \\
\hline NE & Nebraska \\
\hline $\mathrm{NH}$ & New Hampshire \\
\hline NJ & New Jersey \\
\hline NM & New Mexico \\
\hline $\mathrm{NV}$ & Nevada \\
\hline NY & New York \\
\hline $\mathrm{OH}$ & Ohio \\
\hline $\mathrm{OK}$ & Oklahoma \\
\hline OR & Oregon \\
\hline PA & Pennsylvania \\
\hline PR & Puerto Rico \\
\hline RI & Rhode Island \\
\hline $\mathrm{SC}$ & South Carolina \\
\hline SD & South Dakota \\
\hline $\mathrm{TN}$ & Tennessee \\
\hline TX & Texas \\
\hline UM & U.S. Minor Outlying Islands \\
\hline UT & Utah \\
\hline VA & Virginia \\
\hline VI & U.S. Virgin Islands \\
\hline VT & Vermont \\
\hline WA & Washington \\
\hline WI & Wisconsin \\
\hline
\end{tabular}

\begin{tabular}{|c|c|}
\hline StateCode & State \\
\hline WV & West Virginia \\
\hline WY & Wyoming \\
\hline
\end{tabular}

\section{IuStreamOrder}

\begin{tabular}{ccc}
\hline $\begin{array}{c}\text { Stream } \\
\text { OrderlD }\end{array}$ & \multicolumn{1}{c}{ Stream0rder } & $\begin{array}{c}\text { Stream0rder } \\
\text { MethodID }\end{array}$ \\
\hline 1 & $\begin{array}{c}\text { Starting from the headwaters, an } \\
\text { unbranched tributary }\end{array}$ & 1 \\
2 & $\begin{array}{c}\text { Fed by two or more unbranched } \\
\text { tributaries }\end{array}$ & 1 \\
3 & $\begin{array}{c}\text { Fed by two or more of the above } \\
\text { Any other stream (fed by further branched } \\
\text { streams) }\end{array}$ & 1 \\
\hline
\end{tabular}

\section{IuStreamOrderMethodID}

\begin{tabular}{cl}
\hline $\begin{array}{c}\text { StreamOrder } \\
\text { MethodID }\end{array}$ & StreamOrderMethod \\
\hline 1 & Strahler stream order \\
\hline
\end{tabular}

\section{luStructure}

\begin{tabular}{ll}
\hline & Structure \\
\hline Continuous & \\
Intermittent & \\
Open & \\
\hline
\end{tabular}

\section{luStudyType}

\begin{tabular}{l}
\hline \multicolumn{1}{c}{ StudyType } \\
\hline Aquatic species \\
Avian species \\
General management \\
Genetics \\
Habitat restoration \\
Lands management \\
Monitoring-Animals \\
Monitoring-Vegetation \\
Terrestrial Invertebrate \\
Terrestrial Vertebrate \\
Vegetation Surveys
\end{tabular}




\section{luSubstrate}

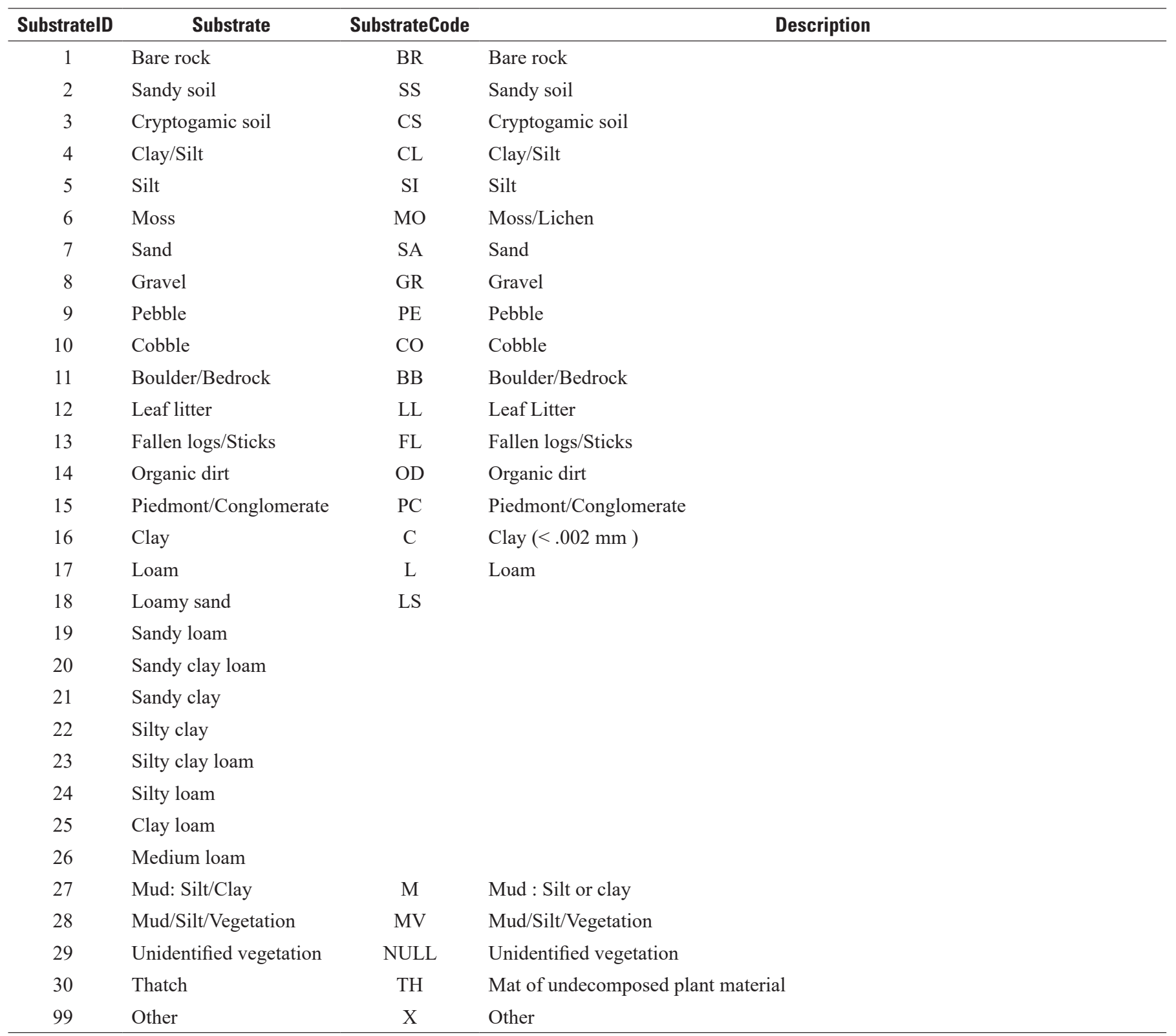

\section{luSubstrateContext}

\begin{tabular}{cll}
\hline SubstrateContextCode & SubstrateContext & Description \\
\hline B & Bank or shore & \\
G & General aquatic & \\
S & Surface & Terrestrial substrate collected at surface \\
SS & Sub-surface & Terrestrial substrate collected at depth \\
T & Terrestrial & \\
\hline
\end{tabular}




\section{IuSuveyableAreaUnit}

\begin{tabular}{ll}
\hline \multicolumn{1}{c}{ SAUnit } \\
\hline Acres \\
Hectares \\
Meters \\
Percent \\
Square feet \\
Square meters \\
Square miles \\
\hline
\end{tabular}

\section{luSurveyMethod}

\begin{tabular}{ll}
\hline SurveyMethod & Description \\
\hline Call survey & \\
Telemetry & \\
Day & \\
Dip net & \\
Radio tracking \\
Visual encounter \\
Other \\
Seine
\end{tabular}

\section{luTaxaRole}

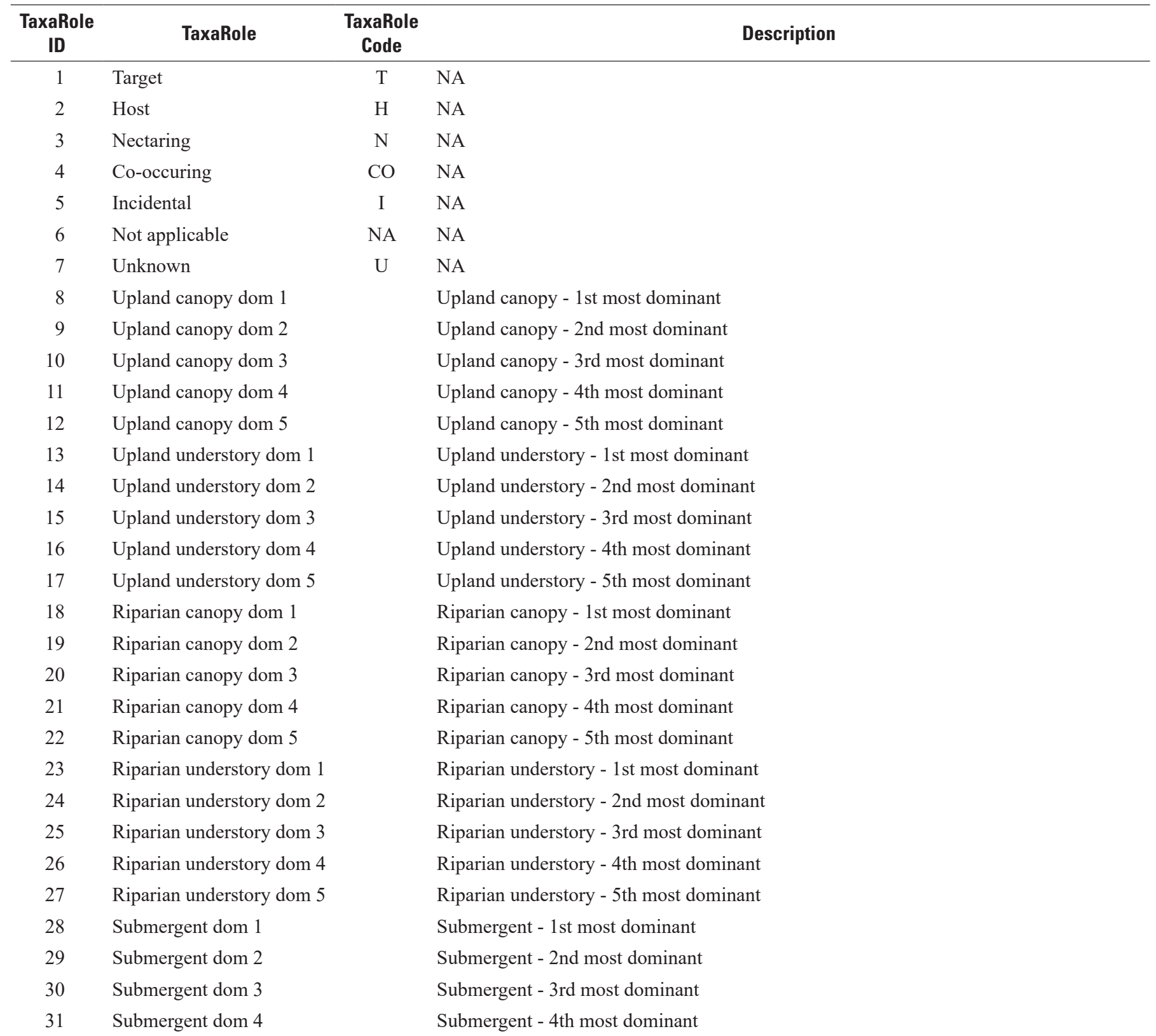




\begin{tabular}{|c|c|c|c|}
\hline $\begin{array}{c}\text { TaxaRole } \\
\text { ID }\end{array}$ & TaxaRole & $\begin{array}{l}\text { TaxaRole } \\
\text { Code }\end{array}$ & \\
\hline 32 & Submergent dom 5 & & Submergent -5 th \\
\hline 33 & Emergent dom 1 & & Emergent - 1st m \\
\hline 34 & Emergent dom 2 & & Emergent - 2nd $n$ \\
\hline 35 & Emergent dom 3 & & Emergent $-3 r d n$ \\
\hline 36 & Emergent dom 4 & & Emergent - 4th $\mathrm{m}$ \\
\hline 37 & Emergent dom 5 & & Emergent -5 th $\mathrm{m}$ \\
\hline 38 & Food source & & Food source \\
\hline 39 & Dominant vegetation & & Most dominant $\mathrm{v}$ \\
\hline \multicolumn{4}{|l|}{ luTeats } \\
\hline TeatsID & \multicolumn{3}{|c|}{ Description } \\
\hline $\mathrm{BA}$ & Bare & & \\
\hline NV & Not visible & & \\
\hline SW & Swollen & & \\
\hline VI & Visible & & \\
\hline
\end{tabular}

\section{luTrapType}

\begin{tabular}{l}
\hline TrapType \\
\hline 1 x 1 foot coverboard \\
2 gallon bucket (pitfall) \\
2 x 2 foot coverboard \\
5 gallon bucket pitfall \\
5 inch funnel trap \\
50 milliliter plastic centrifuge Tubes \\
6 gallon bucket pitfall \\
Box \\
Catfish trap \\
Dog A \\
Dog B \\
Hoop_1.5_fingered \\
Hoop_1.5_singlefingered \\
Hoop_2.5_fingered \\
Hoop_2.5_flat \\
Hoop_3_fingered \\
IR video \\
Large Sherman live trap \\
Med perf Sherman \\
Med Sherman \\
Medium \\
Minnow \\
Oval
\end{tabular}

\begin{tabular}{ll}
\hline IuTrapType-Continued & \\
\hline & TrapType \\
\hline Small & \\
Small mammal (pitfall) \\
Small Sherman \\
Tube 1" \\
Tube 1.5" \\
Tube 2" \\
Turtle trap
\end{tabular}

\section{luTreeSize}

\begin{tabular}{ccc}
\hline TreeSizeID & TreeSize & \multicolumn{1}{c}{ Description } \\
\hline 1 & $<1 \mathrm{DBH}$ & $\begin{array}{c}\text { Less than } 1 \text { inch diameter breast height } \\
(\mathrm{DBH})\end{array}$ \\
2 & $1-6 \mathrm{DBH}$ & $\begin{array}{c}\text { Between } 1 \text { and } 6 \text { inches diameter breast } \\
\text { height }(\mathrm{DBH})\end{array}$ \\
3 & $6-11 \mathrm{DBH}$ & $\begin{array}{c}\text { Between } 6 \text { and } 11 \text { inches diameter } \\
\text { breast height }(\mathrm{DBH})\end{array}$ \\
5 & $11-24 \mathrm{DBH}$ & $\begin{array}{c}\text { Between } 11 \text { and } 24 \text { inches diameter } \\
\text { breast height }(\mathrm{DBH})\end{array}$ \\
6 & Multi-layer & $\begin{array}{c}\text { More than 25 inches diameter breast } \\
\text { height }(\mathrm{DBH})\end{array}$ \\
\hline
\end{tabular}

\section{luVegetationStandSize}

\begin{tabular}{clc}
\hline $\begin{array}{c}\text { Vegetation } \\
\text { StandSizeID }\end{array}$ & \multicolumn{1}{c}{ VegetationStandSize } & $\begin{array}{c}\text { Vegetation } \\
\text { StandSizeCode }\end{array}$ \\
\hline 1 & Very small (<25 sq meters) & VS \\
2 & Small (25-100 sq meters) & $\mathrm{S}$ \\
3 & Medium (100-500 sq meters) & $\mathrm{M}$ \\
4 & Large (500-1000 sq meters) & $\mathrm{L}$ \\
5 & Extra large (>1000 sq meters) & $\mathrm{XL}$ \\
\hline
\end{tabular}




\section{IuVerifMethod}

\begin{tabular}{ll}
\hline & VerifMethod \\
\hline Keyed & \\
Photo comparison & \\
Specimen comparison & \\
\hline
\end{tabular}

\section{IuWaterFlow}

\begin{tabular}{cll}
\hline WaterFlowID & WaterFlow & \multicolumn{1}{c}{ Description } \\
\hline 1 & Dry & No visible moisture/water \\
2 & Moist & Visible moisture without flow or seep \\
3 & Pool & Standing/Stagnant water \\
4 & Riffle & $\begin{array}{c}\text { Small waves (not caused by } \\
\text { obstructions) }\end{array}$
\end{tabular}

\begin{tabular}{|c|c|c|}
\hline WaterFlowID & WaterFlow & Description \\
\hline 5 & Run & $\begin{array}{l}\text { Swiftly-moving, smooth-surface } \\
\text { current }\end{array}$ \\
\hline 6 & Seep & Slow flow, trickle or drip \\
\hline 7 & Splash zone & $\begin{array}{l}\text { Where water splashes against } \\
\text { substrate }\end{array}$ \\
\hline
\end{tabular}

\section{luWaterSource}

\begin{tabular}{cl}
\hline $\begin{array}{c}\text { WaterSource } \\
\text { Code }\end{array}$ & \multicolumn{1}{c}{ WaterSource } \\
\hline $\mathrm{H}$ & Human altered \\
$\mathrm{M}$ & Manmade/Artificial \\
$\mathrm{N}$ & Natural occuring \\
$\mathrm{U}$ & Unknown \\
\hline
\end{tabular}

\section{luWindBeaufortScale}

\begin{tabular}{cccll}
$\begin{array}{c}\text { WindBeaufort } \\
\text { ScaleID }\end{array}$ & MinMPH & MPHRange & \multicolumn{1}{c}{ Description } & \\
\hline 0 & 0 & $<1$ & Calm & Calm; smoke rises vertically. \\
1 & 1 & $1-3$ & Light air & Direction of wind shown by smoke drift, but not by wind vanes \\
2 & 4 & $4-7$ & Light breeze & Wind felt on face; leaves rustle; ordinary vanes moved by wind \\
3 & 8 & $8-12$ & Gentle breeze & Leaves and small twigs in constant motion; wind extends light flag \\
4 & 13 & $13-18$ & Moderate breeze & Raises dust and loose paper; small branches are moved \\
5 & 19 & $19-24$ & Fresh breeze & Small trees in leaf begin to sway; crested wavelets form on inland waters \\
6 & 25 & $25-31$ & Strong breeze & Large branches in motion; whistling heard in telegraph wires; umbrellas used \\
7 & 32 & $32-38$ & Near gale & Whole trees in motion; inconvenience felt when walking against the wind \\
8 & 39 & $39-46$ & Gale & Breaks twigs off trees; generally impedes progress \\
9 & 47 & $47-54$ & Severe gale & Slight structural damage occurs (chimney-pots and slates removed) \\
10 & 55 & $55-63$ & Storm & Seldom experienced inland; trees uprooted; considerable structural damage \\
& & & & occurs \\
11 & 64 & $64-72$ & Violent Storm & Very rarely experienced; accompanied by wide-spread damage \\
12 & 73 & $>=73$ & Hurricane & Hurricane or higher \\
99 & 0 & & Unknown & Unknown \\
\hline
\end{tabular}




\section{luXYCoordType}

\begin{tabular}{|c|c|c|}
\hline XYCoordTypeID & XYCoordType & Description \\
\hline 1 & UTM zone $11 \mathrm{~S}$ & \\
\hline 2 & Decimal degrees & \\
\hline 3 & UTM zone 11 north & \\
\hline 4 & UTM zone $10 \mathrm{~T}$ & \\
\hline 5 & UTM zone $12 \mathrm{R}$ & \\
\hline 6 & UTM zone $15 \mathrm{R}$ & \\
\hline 7 & UTM zone $17 \mathrm{R}$ & \\
\hline 8 & UTM zone $10 \mathrm{U}$ & \\
\hline 9 & UTM zone $10 \mathrm{~S}$ & \\
\hline 10 & UTM zone $11 \mathrm{U}$ & \\
\hline 11 & UTM zone $11 \mathrm{~T}$ & \\
\hline 12 & UTM zone $12 \mathrm{U}$ & \\
\hline 13 & UTM zone $12 \mathrm{~T}$ & \\
\hline 14 & UTM zone $12 \mathrm{~S}$ & \\
\hline 15 & UTM zone $13 \mathrm{U}$ & \\
\hline 16 & UTM zone $13 \mathrm{~T}$ & \\
\hline 17 & UTM zone $13 \mathrm{~S}$ & \\
\hline 18 & UTM zone $13 \mathrm{R}$ & \\
\hline 19 & UTM zone $14 \mathrm{U}$ & \\
\hline 20 & UTM zone $14 \mathrm{~T}$ & \\
\hline 21 & UTM zone $14 \mathrm{~S}$ & \\
\hline 22 & UTM zone $14 \mathrm{R}$ & \\
\hline 23 & UTM zone $15 \mathrm{~T}$ & \\
\hline 24 & UTM zone $15 \mathrm{~S}$ & \\
\hline 25 & UTM zone $15 \mathrm{U}$ & \\
\hline 26 & UTM zone $16 \mathrm{~T}$ & \\
\hline 27 & UTM zone $16 \mathrm{~S}$ & \\
\hline 28 & UTM zone $16 \mathrm{R}$ & \\
\hline 29 & UTM zone $17 \mathrm{~T}$ & \\
\hline 30 & UTM zone $17 \mathrm{~S}$ & \\
\hline 31 & UTM zone $18 \mathrm{~T}$ & \\
\hline 32 & UTM zone $18 \mathrm{~S}$ & \\
\hline 33 & UTM zone 19T & \\
\hline
\end{tabular}

\section{Permanency}

\begin{tabular}{cl}
\hline PID & \multicolumn{1}{c}{ Permanency } \\
\hline P & Permanent \\
S & Semi-permanent (rarely dries) \\
$\mathrm{T}$ & Temporary (dries annually) \\
$\mathrm{U}$ & Unknown \\
\hline
\end{tabular}

\section{TissType}

\begin{tabular}{cl}
\hline TissueTypelD & \multicolumn{1}{c}{ TissueDesc } \\
\hline 01 & Pattern recognition \\
02 & Pit tag \\
03 & Scaleclip \\
05 & Toeclip/Tailclip \\
06 & VIE \\
07 & Muscle \\
08 & Liver \\
09 & Heart \\
10 & Skeletal \\
11 & Whole \\
12 & Earclip \\
97 & Other \\
99 & Unknown \\
\hline
\end{tabular}

\section{Transparency}

\begin{tabular}{cl}
\hline TransplD & \multicolumn{1}{c}{ Transparency } \\
\hline $\mathrm{C}$ & Clear \\
$\mathrm{M}$ & Moderate/Translucent \\
$\mathrm{O}$ & Opaque \\
\hline
\end{tabular}

\section{TranspMethod}

\begin{tabular}{cl}
\hline TranspMID & \multicolumn{1}{c}{ TranspMethod } \\
\hline 1 & Ruler \\
2 & Secchi disk \\
3 & Transparency tube \\
4 & Visual estimate \\
\hline
\end{tabular}




\section{TurbUnits}

\begin{tabular}{cll}
\hline TurbUID & & TurbUnits \\
\hline 1 & NTU's & \\
2 & FTU's & \\
\hline
\end{tabular}

UnitEffort

\begin{tabular}{cl}
\hline UnitEffortID & \multicolumn{1}{c}{ UnitEffort } \\
\hline 01 & Artificial cover objects \\
02 & Dipnet sweeps \\
03 & Dredge scoops \\
04 & Drift fence arrays \\
05 & Funnel traps \\
06 & Leaf litter bags \\
07 & Minnow traps \\
08 & Natural cover objects \\
09 & Night driving distance (km) \\
10 & Pitfall traps \\
11 & PVC pipe \\
12 & Seine pulls \\
13 & Minutes (visual search) \\
14 & GPS collar days \\
15 & Remote camera days \\
16 & Track station nights \\
17 & Minutes (audible search) \\
18 & Aquatic trap days \\
19 & Minutes (visual and audible) \\
20 & Cowbird trap days \\
21 & Hectares visual search \\
22 & Fence installation/repair (km) \\
23 & Habitat restoration (hec) \\
& \\
\hline 1 &
\end{tabular}

\begin{tabular}{cl}
\hline UnitEffortID & \multicolumn{1}{c}{ UnitEffort } \\
\hline 24 & Vegetation point transect \\
99 & Not applicable \\
\hline
\end{tabular}

\section{WaterColor}

WaterColor

Clear

Stained 


\section{Appendix 1. Entity Relationship Diagram of All Database Tables}

File available for download from https://doi.org/10.3133/tm16B1.

\section{Appendix 2. Entity Relationship Diagram of Tables Associated with Survey Events}

File available for download from https://doi.org/10.3133/tm16B1.

\section{Appendix 3. Entity Relationship Diagram of Tables Associated with Sites}

File available for download from https://doi.org/10.3133/tm16B1.

\section{Appendix 4. Entity Relationship Diagram of Tables Associated with Taxa Observations}

File available for download from https://doi.org/10.3133/tm16B1.

\section{Appendix 5. Entity Relationship Diagram of Tables Associated with Habitat Observations}

File available for download from https://doi.org/10.3133/tm16B1. 

For more information concerning the research in this report, contact the

Director, Western Ecological Research Center

U.S. Geological Survey

3020 State University Drive East

Sacramento, California 95819

https://www.usgs.gov/centers/werc

Publishing support provided by the U.S. Geological Survey

Science Publishing Network, Sacramento Publishing Service Center 


\section{$\frac{1}{4}$}

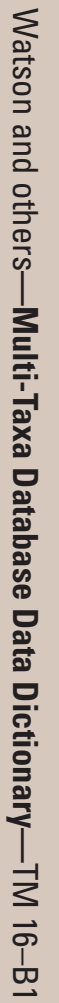

